\title{
Clinical cardiac computed tomographic angiography : implications for risk stratification
}

Citation for published version (APA):

Versteylen, M. O. (2013). Clinical cardiac computed tomographic angiography : implications for risk stratification. [Doctoral Thesis, Maastricht University]. Datawyse / Universitaire Pers Maastricht. https://doi.org/10.26481/dis.20131120mv

Document status and date:

Published: 01/01/2013

DOI:

10.26481/dis.20131120mv

Document Version:

Publisher's PDF, also known as Version of record

\section{Please check the document version of this publication:}

- A submitted manuscript is the version of the article upon submission and before peer-review. There can be important differences between the submitted version and the official published version of record.

People interested in the research are advised to contact the author for the final version of the publication, or visit the DOI to the publisher's website.

- The final author version and the galley proof are versions of the publication after peer review.

- The final published version features the final layout of the paper including the volume, issue and page numbers.

Link to publication

\footnotetext{
General rights rights.

- You may freely distribute the URL identifying the publication in the public portal. please follow below link for the End User Agreement:

www.umlib.nl/taverne-license

Take down policy

If you believe that this document breaches copyright please contact us at:

repository@maastrichtuniversity.nl

providing details and we will investigate your claim.
}

Copyright and moral rights for the publications made accessible in the public portal are retained by the authors and/or other copyright owners and it is a condition of accessing publications that users recognise and abide by the legal requirements associated with these

- Users may download and print one copy of any publication from the public portal for the purpose of private study or research.

- You may not further distribute the material or use it for any profit-making activity or commercial gain

If the publication is distributed under the terms of Article $25 \mathrm{fa}$ of the Dutch Copyright Act, indicated by the "Taverne" license above, 


\section{Clinical cardiac computed tomographic angiography \\ -implications for risk stratification-}


(C) Mathijs O. Versteylen, Maastricht 2013

ISBN 9789461592736

Cover design: Roy Maassen

Production: Datawyse Maastricht 


\title{
Clinical cardiac computed tomographic angiography -implications for risk stratification-
}

\author{
PROEFSCHRIFT
}

ter verkrijging van de graad van doctor aan de Universiteit Maastricht, op gezag van de Rector Magnificus, Prof. dr. L.L.G. Soete, volgens besluit van het College van Decanen, in het openbaar te verdedigen op woensdag 20 november 2013 om 12:00 uur

door

Mathijs Olivier Versteylen

Geboren op 28 april 1984 te Rotterdam

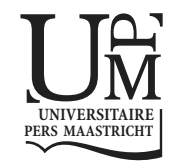




\section{Promotores}

Prof. dr. H.J.G.M. Crijns

Prof. dr. L. Hofstra

\section{Co-promotores}

Dr. M. Das

Dr. B.L.J.H. Kietselaer

\section{Beoordelingscommissie}

Prof. dr. H.-P. Brunner-La Rocca (voorzitter/chair)

Dr. J. Bucerius

Prof. dr. M.W. de Haan

Prof. dr. M. Oudkerk (UMC Groningen)

Dr. L. Schurgers

The work described in this thesis was partially funded by CTMM, the Center for Translational Molecular Medicine (www.ctmm.nl), project PARISk (grant 01C-202), and supported by the Dutch Heart Foundation.

Financial support by 'stichting hartsvrienden RESCAR Maastricht' for the publication of this thesis is gratefully acknowledged. Additional financial support was generously provided by: Cardiovascular Imaging and Research Center (CIRC), Astellas BV, Merck Sharp \& Dohme BV, Guerbet Nederland BV, Bayer BV and Philips Healthcare. 


\section{Contents}

$\begin{array}{lll}\text { Chapter } 1 & \text { Introduction } & 7\end{array}$

Chapter 2 Comparison of Framingham, PROCAM, SCORE and Diamond 19

Forrester to predict coronary atherosclerosis and cardiovascular events

Chapter 3 Epicardial adipose tissue volume as a predictor for coronary artery disease in diabetic, impaired fasting glucose and nondiabetic patients presenting with chest pain

Chapter 4 Coronary anomalies with and without an inter-arterial course as detected by cardiac computed tomographic angiography: differences in coronary dimensions and clinical follow-up?

Chapter 5 Combined use of exercise electrocardiography, coronary calcium score and cardiac CT angiography for the prediction of major cardiovascular events in patients presenting with stable chest pain

Chapter 6 Gender difference in prognostic value of cardiac computed tomographic angiography?

Chapter 7 Additive value of semiautomated quantification of coronary artery disease using cardiac computed tomographic angiography to predict future acute coronary syndrome

Editorial by E. Shapiro and D. Bush: Visualizing Vulnerability Toward a New Cardiac Score

Chapter 8 CC-chemokine ligands in patients presenting with stable chest pain: association with atherosclerosis and future cardiovascular events 
Chapter 9 General discussion

Summary

175

Nederlandse samenvatting

177

Nawoord

179

Curriculum vitae

183

List of publications

185 


\section{Chapter 1}

General introduction 


\section{Cardiovascular disease}

Cardiovascular disease is the leading cause of death globally, including Western Europe. ${ }^{1}$ The cardiovascular disease epidemic is expected to rise even further and it is forecasted that by 2030, 40.5\% of the United States population will be affected. ${ }^{2}$ Also developing countries show a rapid increase in atherosclerotic burden, with more expected cardiovascular deaths in India or China than in all developed countries added together by $2030 .^{3}$ The major cause of cardiovascular death is coronary artery disease. ${ }^{4}$ Moreover, ischemic heart disease is expected to be the most important cause of lost of healthy life years in $2020 .^{5}$ Because of the high impact on our patients and society, diagnosing coronary artery disease is one of the cornerstones of clinical cardiology. Since atherosclerosis has a progressive character, focus should be on prevention as well as early detection of disease in order to deal with this major health care problem. The challenge is finding a balance between early diagnosing and high costs involved. This forces us to pursue effective diagnostic techniques and to align existing resources in the most effective manner.

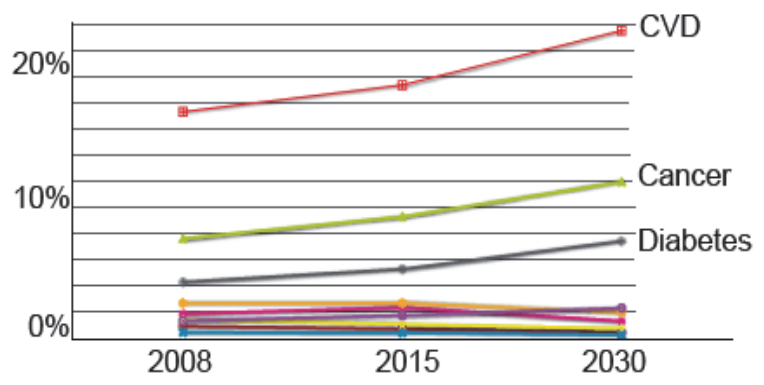

Figure 1. Mortality trends projected to 2030. WHO 2011

\section{Chest pain}

Coronary artery disease can result in obstruction of the coronary lumen, leading to ischemia and even infarction of the myocardium. ${ }^{6}$ From a classical point of view, the obstruction is thought to gradually aggravate. Accordingly, nonobstructive coronary artery stenosis does not yet lead to inadequate blood flow and symptoms. In case of a more severe obstruction and/or a higher myocardial oxygen demand, the myocardium supplied by the affected coronary artery can become ischemic. 7,8 As a result, patients can experience symptoms of chest pain during exercise. These symptoms are known as stable chest pain, as they are refractory after cessation of exercise. Eventually, an aggravating coronary obstruction will also produce symptoms during rest. Subsequently, the myocar- 
dium becomes ischemic or even infarcted, which causes irreversible damage to the heart and can result in arrhythmias and death.

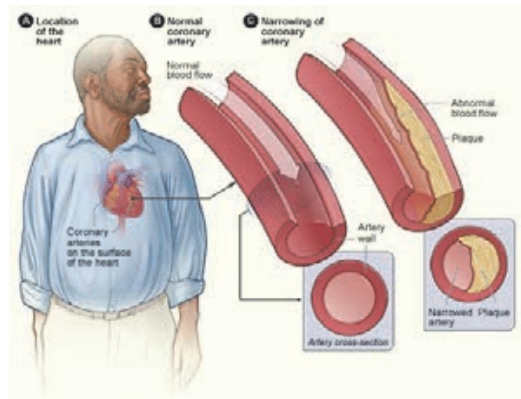

Figure 2 A. Coronary stenosis NHLBI public domain

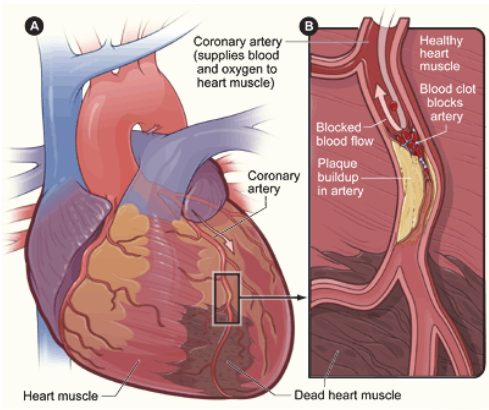

Figure 2 B. Myocardial infarction.

\section{Coronary events}

The majority of coronary events are caused by rupture of a non-obstructive plaque, ${ }^{9}$ leading to acute thrombus formation and obstruction of the coronary artery. ${ }^{10}$ Pathology studies have suggested that certain plaques are more vulnerable to plaque rupture. ${ }^{11}$ Consequently, not only stenosis severity, but also plaque characteristics and composition have important prognostic implications.

The majority of patients presenting with myocardial infarction have reported chest pain symptoms prior to their event. However, since coronary obstruction is not imperative to develop a coronary event, in a large amount of patients a myocardial infarction is the first symptom of coronary artery disease. It is even estimated that more than $30 \%$ of all sudden cardiac deaths in the United States are the first presentation of coronary artery disease. ${ }^{12}$ Hence, the concept of the vulnerable plaque better suits these clinical observations, and has gained popularity in during the last decades. Nevertheless, it is concerning that apparently, conventional diagnostic techniques cannot accurately predict the risk of myocardial infarction in a large amount of patients. 


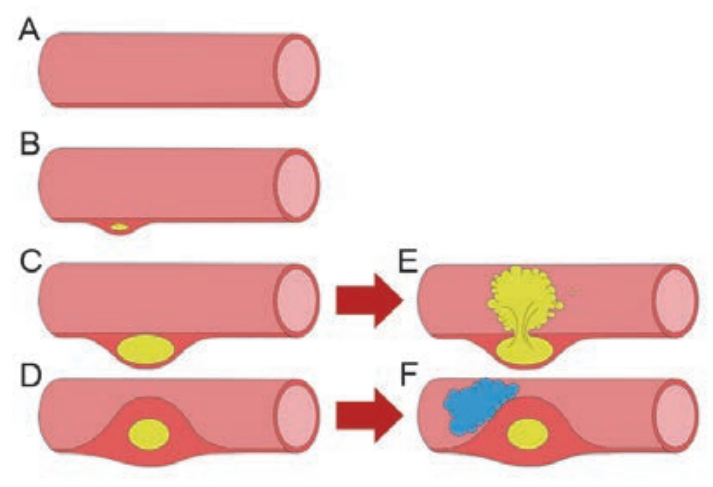

Figure 3. Development of atherosclerotic plaque and coronary events. A: normal coronary artery. B: small plaque development. C: outward remodeled plaque. D: stenotic plaque. E: plaque rupture with acute occlusion. F: stenotic plaque with coronary occlusion.

\section{Diagnosing coronary artery disease}

Traditionally, there have been several options to evaluate patients with stable chest pain. One of the most common initial diagnostic test is a rest electrocardiogram followed by exercise electrocardiography. Introducing physical exercise will lead to an increased demand of oxygen in cardiac muscle cells, which results in myocardial ischemia when a functionally significant coronary artery obstruction is present. However, it is known that the diagnostic performance of exercise electrocardiography is limited, and often produces false negative and false positive results. ${ }^{13,} 14$ In addition, nuclear imaging techniques are especially designed to detect myocardial ischemia. Using radioactive tracers, for instance bound to methoxyisobutylisonitrile (MIBI), perfusion of the myocardium can be imaged using single photon emission computed tomography (SPECT). MIBI-SPECT has a high sensitivity to evaluate functional significance of a coronary lesion..$^{15}$ Also, stress echocardiography can detect wall motion abnormalities indicating ischemia and/or myocardial infarction 16, and cardiovascular magnetic resonance imaging is an emerging technique to detect ischemia and presence of infarction. ${ }^{17}$ The overall limitation of these techniques might be the fact that, although ischemia is a derivate of significant coronary stenosis, it does not provide direct insight into the presence of coronary artery disease. The current gold standard technique for detecting coronary artery disease is considered to be conventional coronary angiography. This invasive technique visualizes the lumen of the coronary artery system, and has a risk of major complications of $<2 \% .{ }^{18},{ }^{19}$ It has to be noted that a relatively high percentage of chest pain patients have normal conventional coronary angiograms. ${ }^{20}$ The technique is able to visualize the coronary lumen, but it does not provide information 
about the vessel wall. Consequently, it provides information on coronary obstruction, but not on coronary plaque characteristics.

\section{Cardiac computed tomographic angiography}

Cardiac computed tomographic angiography (CCTA) has emerged as a new technique to evaluate coronary artery disease. ${ }^{21-24}$ During the last decades, CT scanners have become faster, subsequently resulting in lower acquisition time and radiation dose. This allowed the technique to visualize the coronary arteries of the moving heart, using intravascular contrast and ECG-gating to produce still images. CCTA is able to visualize all structures within scan range. Therefore, in addition to the contrast enhanced coronary lumen, it provides information about the vessel wall, the myocardium, and remaining cardiac anatomy. Acquisition is fast and patient friendly, which makes the technique

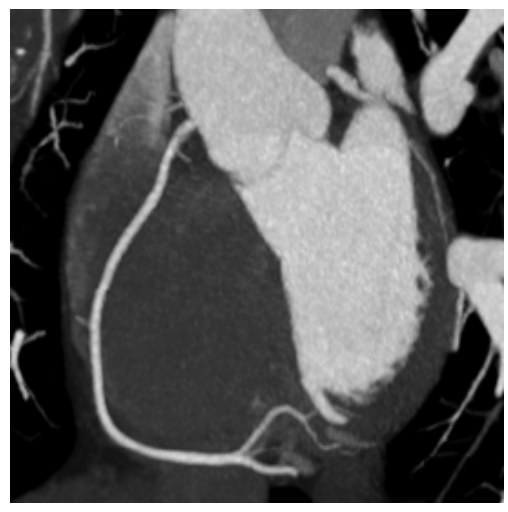

Figure 4. Cardiac computed tomographic angiography of the right coronary artery. easily applicable for clinical use. The greatest concern is the increment in radiation dose CCTA might deliver to the population. ${ }^{25}$ However, rapid advances in CT technique, especially great improvement in temporal resolution, are accompanied by a substantial decrease in radiation dose. For instance, the introduction of dual-source CT scanners enables CCTA acquisition at very low dose..$^{26,27}$

\section{Diagnostic and prognostic value of CCTA}

The diagnostic accuracy of CCTA, as compared to the gold standard conventional coronary angiography, is well established. Especially sensitivity and negative predictive value of the technique are high. ${ }^{28,} 29$ Specificity has been more problematic, in particular evaluating highly calcified coronary artery disease is difficult, predominantly due to 'blooming' or overestimation of the calcified plaques. ${ }^{30}$ Therefore, CCTA has been considered a 'rule-out' tool for coronary artery disease and is recommended in low- to intermediate risk patients presenting with stable chest pain. ${ }^{31}$

In addition, more recent studies reported on the prognostic value of CCTA. Most studies focused on the prognostic implications of a $\geq 50 \%$ steno- 
sis. ${ }^{32-35}$ From a classical point of view, angiographic stenosis severity has been crucial in determining prognosis of the individual patient. Using CCTA however, additional information about plaque morphology and geometry are also available.

Several studies have investigated the ability of CCTA to identify plaque characteristics, using intravascular ultrasound as the gold standard. ${ }^{36,37}$ Especially mixed and calcified lesions can be accurately detected by CCTA, noncalcified plaques were harder to detect. 38,39 The differentiation between fibrous tissue en lipid rich core using CCTA remains problematic. ${ }^{40}$ Further, multiple studies have been performed on the association between plaque characteristics and the occurrence of coronary events. ${ }^{41,}{ }^{42}$ Pathologic characteristics of vulnerable plaques were investigated using CCTA, and especially outward remodeled plaques, spotty calcification and low plaque attenuation were associated with ACS. $.3,44$ Also, plaque volume seems to be an important predictor for plaque vulnerability. 45

Thus, the clinical use of CCTA can enable clinicians to visualize nonobstructive lesions, and may thereby image atherosclerosis at an early state. The technique seems to behold important prognostic information, and may be of potential aid in risk-stratifying patients for cardiovascular events. Hence, CCTA might improve the identification of high risk coronary artery disease beyond stenosis severity. Consequently, it is essential to study the potential increase in prognostic power that coronary plaque characteristics might behold. In times of rapidly increasing health care costs, the greatest challenge we face is to apply diagnostic techniques in a selective as well as effective manner. In other words, we have to find out in which patients a test is most effective, when to perform this test, and how to obtain the most information from it as possible. With the classical focus on stenosis severity of coronary artery disease, not all information provided by imaging datasets is used, thereby not fully exploiting the potential of CCTA.

\section{Thesis outline}

In chapter 2 we will investigate the relation between simple clinical risk factors and the presence of coronary artery disease as visualized by CCTA. The performance of several clinical risk scores is investigated. Most guidelines advise CCTA in stable chest pain patients with intermediate risk profiles. Using this

principle, we sought to determine the most effective clinical risk profiling algorithm to use prior to CCTA. 
In chapter 3, epicardial adipose tissue will be evaluated as a marker for coronary artery disease. It has been hypothesized that perivascular adipose tissue has atherogenic effects. Therefore, it might be an attractive risk stratification tool for coronary artery disease. The use of this potential risk factor is investigated in different subgroups. The detection of coronary anomalies by CCTA and their short-term follow-up is investigated in chapter 4. Subsequently, we report on the combined use of exercise ECG, calcium score and CCTA to predict for cardiac events in chapter 5 . We investigate the relative prognostic value of these techniques, and evaluate the value of several combinations. In chapter 6, we study the potential difference in prognostic value of CCTA in men and women. In chapter 7 , we will investigate coronary plaque characteristics on CCTA and the subsequent development of acute coronary syndromes. We will use a semi-automated quantification algorithm to identify high-risk plaque characteristics as detected by CCTA. The additive value of using such an algorithm over conventional CCTA reading (i.e. calcium score and stenosis severity) is further investigated. Finally, the use of serum chemokines CCL3, 5 and 18 to predict for atherosclerosis and cardiac events is explored in chapter 8.

\section{References}

1. Lopez AD, Mathers CD, Ezzati M, Jamison DT, Murray CJ. Global and regional burden of disease and risk factors, 2001: systematic analysis of population health data. Lancet 2006;367:1747-57.

2. Heidenreich PA, Trogdon JG, Khavjou OA, et al. Forecasting the future of cardiovascular disease in the United States: a policy statement from the American Heart Association. Circulation 2011;123:933-44.

3. Patel V, Chatterji S, Chisholm D, et al. Chronic diseases and injuries in India. Lancet 2011;377:413-28.

4. Roger VL, Go AS, Lloyd-Jones DM, et al. Heart disease and stroke statistics--2011 update: a report from the American Heart Association. Circulation 2011;123:e18-e209.

5. Murray CJ, Lopez AD. Alternative projections of mortality and disability by cause 19902020: Global Burden of Disease Study. Lancet 1997;349:1498-504.

6. Reimer KA, Lowe JE, Rasmussen MM, Jennings RB. The wavefront phenomenon of ischemic cell death. 1. Myocardial infarct size vs duration of coronary occlusion in dogs. Circulation 1977;56:786-94. 
7. Schroder K, Schultheiss HP. Coronary artery disease--diagnosis of ischaemia: general considerations. Eur Heart J 1997;18 Suppl D:D57-62.

8. Gibbons RJ, Chatterjee K, Daley J, et al. ACC/AHA/ACP-ASIM guidelines for the management of patients with chronic stable angina: executive summary and recommendations. A Report of the American College of Cardiology/American Heart Association Task Force on Practice Guidelines (Committee on Management of Patients with Chronic Stable Angina). Circulation 1999;99:2829-48.

9. Ambrose JA, Tannenbaum MA, Alexopoulos D, et al. Angiographic progression of coronary artery disease and the development of myocardial infarction. J Am Coll Cardiol 1988;12:56-62.

10. Virmani R, Burke AP, Farb A, Kolodgie FD. Pathology of the vulnerable plaque. J Am Coll Cardiol 2006;47:C13-8.

11. Finn AV, Nakano M, Narula J, Kolodgie FD, Virmani R. Concept of vulnerable/unstable plaque. Arterioscler Thromb Vasc Biol 2010;30:1282-92.

12. Myerburg RJ, Junttila MJ. Sudden cardiac death caused by coronary heart disease. Circulation 2012;125:1043-52.

13. Gibbons RJ, Balady GJ, Bricker JT, et al. ACC/AHA 2002 guideline update for exercise testing: summary article: a report of the American College of Cardiology/American Heart Association Task Force on Practice Guidelines (Committee to Update the 1997 Exercise Testing Guidelines). Circulation 2002;106:1883-92.

14. Braunwald E, Antman EM, Beasley JW, et al. ACC/AHA 2002 guideline update for the management of patients with unstable angina and non-ST-segment elevation myocardial infarction--summary article: a report of the American College of Cardiology/American Heart Association task force on practice guidelines (Committee on the Management of Patients With Unstable Angina). J Am Coll Cardiol 2002;40:1366-74.

15. Jaarsma C, Leiner T, Bekkers SC, et al. Diagnostic performance of noninvasive myocardial perfusion imaging using single-photon emission computed tomography, cardiac magnetic resonance, and positron emission tomography imaging for the detection of obstructive coronary artery disease: a meta-analysis. J Am Coll Cardiol 2012;59:1719-28.

16. Fox K, Garcia MA, Ardissino D, et al. Guidelines on the management of stable angina pectoris: executive summary: The Task Force on the Management of Stable Angina Pectoris of the European Society of Cardiology. Eur Heart J 2006;27:1341-81.

17. Kim RJ, Fieno DS, Parrish TB, et al. Relationship of MRI delayed contrast enhancement to irreversible injury, infarct age, and contractile function. Circulation 1999;100:1992-2002.

18. Scanlon PJ, Faxon DP, Audet AM, et al. ACC/AHA guidelines for coronary angiography: executive summary and recommendations. A report of the American College of Cardiology/American Heart Association Task Force on Practice Guidelines (Committee on Coronary Angiography) developed in collaboration with the Society for Cardiac Angiography and Interventions. Circulation 1999;99:2345-57. 
19. West R, Ellis G, Brooks N. Complications of diagnostic cardiac catheterisation: results from a confidential inquiry into cardiac catheter complications. Heart 2006;92:810-4.

20. Kemp HG, Kronmal RA, Vlietstra RE, Frye RL. Seven year survival of patients with normal or near normal coronary arteriograms: a CASS registry study. J Am Coll Cardiol 1986;7:479-83.

21. Achenbach S, Ulzheimer S, Baum U, et al. Noninvasive coronary angiography by retrospectively ECG-gated multislice spiral CT. Circulation 2000;102:2823-8.

22. Nieman K, Oudkerk M, Rensing BJ, et al. Coronary angiography with multi-slice computed tomography. Lancet 2001;357:599-603.

23. Hoffmann MH, Shi H, Schmitz BL, et al. Noninvasive coronary angiography with multislice computed tomography. JAMA 2005;293:2471-8.

24. Schroeder S, Achenbach S, Bengel F, et al. Cardiac computed tomography: indications, applications, limitations, and training requirements: report of a Writing Group deployed by the Working Group Nuclear Cardiology and Cardiac CT of the European Society of Cardiology and the European Council of Nuclear Cardiology. Eur Heart J 2008;29:531-56.

25. Fazel R, Krumholz HM, Wang Y, et al. Exposure to low-dose ionizing radiation from medical imaging procedures. N Engl J Med 2009;361:849-57.

26. Lell M, Marwan M, Schepis T, et al. Prospectively ECG-triggered high-pitch spiral acquisition for coronary CT angiography using dual source CT: technique and initial experience. Eur Radiol 2009;19:2576-83.

27. Achenbach S, Marwan M, Ropers D, et al. Coronary computed tomography angiography with a consistent dose below $1 \mathrm{mSv}$ using prospectively electrocardiogram-triggered highpitch spiral acquisition. Eur Heart J 2010;31:340-6.

28. Budoff MJ, Dowe D, Jollis JG, et al. Diagnostic performance of 64-multidetector row coronary computed tomographic angiography for evaluation of coronary artery stenosis in individuals without known coronary artery disease: results from the prospective multicenter ACCURACY (Assessment by Coronary Computed Tomographic Angiography of Individuals Undergoing Invasive Coronary Angiography) trial. J Am Coll Cardiol 2008;52:1724-32.

29. Miller JM, Rochitte CE, Dewey M, et al. Diagnostic performance of coronary angiography by 64-row CT. N Engl J Med 2008;359:2324-36.

30. Zhang S, Levin DC, Halpern EJ, Fischman D, Savage M, Walinsky P. Accuracy of MDCT in assessing the degree of stenosis caused by calcified coronary artery plaques. AJR Am J Roentgenol 2008;191:1676-83. 
31. Taylor AJ, Cerqueira M, Hodgson JM, et al. ACCF/SCCT/ACR/AHA/ASE/ASNC/NASCI/SCAI/SCMR 2010 appropriate use criteria for cardiac computed tomography: a report of the American College of Cardiology Foundation Appropriate Use Criteria Task Force, the Society of Cardiovascular Computed Tomography, the American College of Radiology, the American Heart Association, the American Society of Echocardiography, the American Society of Nuclear Cardiology, the North American Society for Cardiovascular Imaging, the Society for Cardiovascular Angiography and Interventions, and the Society for Cardiovascular Magnetic Resonance. J Am Coll Cardiol 2010;56:1864-94.

32. Min JK, Shaw LJ, Devereux RB, et al. Prognostic value of multidetector coronary computed tomographic angiography for prediction of all-cause mortality. J Am Coll Cardiol 2007;50:1161-70.

33. Hadamitzky M, Freissmuth B, Meyer T, et al. Prognostic value of coronary computed tomographic angiography for prediction of cardiac events in patients with suspected coronary artery disease. JACC Cardiovasc Imaging 2009;2:404-11.

34. Min JK, Dunning A, Lin FY, et al. Age- and sex-related differences in all-cause mortality risk based on coronary computed tomography angiography findings results from the International Multicenter CONFIRM (Coronary CT Angiography Evaluation for Clinical Outcomes: An International Multicenter Registry) of 23,854 patients without known coronary artery disease. J Am Coll Cardiol 2011;58:849-60.

35. Dedic A, Genders TS, Ferket BS, et al. Stable angina pectoris: head-to-head comparison of prognostic value of cardiac CT and exercise testing. Radiology 2011;261:428-36.

36. Pundziute G, Schuijf JD, Jukema JW, et al. Head-to-head comparison of coronary plaque evaluation between multislice computed tomography and intravascular ultrasound radiofrequency data analysis. JACC Cardiovasc Interv 2008;1:176-82.

37. Leber AW, Knez A, von Ziegler F, et al. Quantification of obstructive and nonobstructive coronary lesions by 64-slice computed tomography: a comparative study with quantitative coronary angiography and intravascular ultrasound. J Am Coll Cardiol 2005;46:147-54.

38. Leber AW, Becker A, Knez A, et al. Accuracy of 64-slice computed tomography to classify and quantify plaque volumes in the proximal coronary system: a comparative study using intravascular ultrasound. J Am Coll Cardiol 2006;47:672-7.

39. Sun J, Zhang Z, Lu B, et al. Identification and quantification of coronary atherosclerotic plaques: a comparison of 64-MDCT and intravascular ultrasound. AJR Am J Roentgenol 2008;190:748-54.

40. Hur J, Kim YJ, Lee HJ, et al. Quantification and characterization of obstructive coronary plaques using 64-slice computed tomography: a comparison with intravascular ultrasound. J Comput Assist Tomogr 2009;33:186-92.

41. Feuchtner G, Postel T, Weidinger F, et al. Is there a relation between non-calcifying coronary plaques and acute coronary syndromes? A retrospective study using multislice computed tomography. Cardiology 2008;110:241-8. 
42. Kitagawa T, Yamamoto H, Horiguchi J, et al. Characterization of noncalcified coronary plaques and identification of culprit lesions in patients with acute coronary syndrome by 64-slice computed tomography. JACC Cardiovasc Imaging 2009;2:153-60.

43. Motoyama S, Kondo T, Sarai M, et al. Multislice computed tomographic characteristics of coronary lesions in acute coronary syndromes. J Am Coll Cardiol 2007;50:319-26.

44. Motoyama S, Sarai M, Harigaya H, et al. Computed tomographic angiography characteristics of atherosclerotic plaques subsequently resulting in acute coronary syndrome. J Am Coll Cardiol 2009;54:49-57.

45. Pflederer T, Marwan M, Schepis T, et al. Characterization of culprit lesions in acute coronary syndromes using coronary dual-source CT angiography. Atherosclerosis;211:437-44. 



\section{Chapter}

\section{Comparison of Framingham, PROCAM, SCORE and Diamond Forrester to Predict Coronary Atherosclerosis and Cardiovascular Events \\ Mathijs O. Versteylen, Ivo A. Joosen, Leslee J. Shaw, Jagat Narula, Leonard Hofstra}

J Nucl Cardiol. 2011;18:904-11. 


\begin{abstract}
Background Cardiologists are often confronted with patients presenting with chest pain, in whom clinical risk profiling is required. We studied four frequently used risk scores in their ability to predict for coronary artery disease (CAD) and major adverse cardiovascular events in patients presenting with stable chest pain at the cardiology outpatient clinic.

Methods We enrolled 1,296 stable chest pain patients, who underwent cardiac computed tomographic angiography (CCTA) to assess CAD (any, significant: stenosis $\geq 50 \%$ ). Framingham (FRS), PROCAM, SCORE risk score and Diamond Forrester pre-test probability were calculated. All patients were followed up for a mean $19 \pm 9$ months for all cardiovascular events (mortality, acute coronary syndrome, revascularization $>90$ days after CCTA).
\end{abstract}

Results In ROC-analysis for prediction of significant CAD, the areas under the curve for FRS; 0.68 (95\% confidence interval: 0.64 - 0.72) and for SCORE; 0.69 (95\% confidence interval: 0.65 - 0.72) were significantly higher than for PROCAM; 0.64 (95\% confidence interval: 0.61 - 0.68; $\mathrm{p} \leq 0.001$ ), as well as marginally higher than for Diamond Forrester; 0.65 (95\% confidence interval: 0.61 $0.68 ; \mathrm{p} \leq 0.05)$. Low FRS category showed the lowest number of patients with significant CAD, compared to patients with low risk using PROCAM, SCORE or Diamond Forrester $(\mathrm{p}<0.001)$. Also, low FRS category showed less events (compared to PROCAM and SCORE; $\mathrm{p}<0.001$, for Diamond Forrester; $\mathrm{p}=0.14$ ).

Conclusion Our data show that in a stable chest pain population, the ability of FRS and SCORE to predict for CAD was similar and better compared to PROCAM and Diamond Forrester. The number of low risk patients showing significant CAD or events was lower using FRS. Consequently, risk categorization using FRS seems to be safest to stratify stable chest pain patients prior to CCTA. 


\section{INTRODUCTION}

Cardiovascular disease (CVD) is the leading cause of death for both men and women in the United States. ${ }^{1}$ The CVD epidemic is expected to rise even further and it is forecasted that by $2030,40.5 \%$ of the US population will have some form of CVD. ${ }^{2}$ Globally, the atherosclerotic burden is increasing in an even more rapid pace, with more expected CVD deaths in India or China than in all developed countries added together by $2030.3^{3}$

In clinical practice, physicians are often confronted with patients presenting with chest pain, in whom clinical risk profiling is required. ${ }^{4,5}$ Recently, cardiac computed tomographic angiography (CCTA) has been introduced in the clinical workup of chest pain patients. The excellent negative predictive value of this technique makes it most suitable to rule out coronary artery disease (CAD). ${ }^{6}$ Although initial reports on diagnostic accuracy and prognostic value of CCTA are very promising, there are disadvantages such as the substantial radiation dose and administration of contrast agent. Therefore, most guidelines and appropriateness criteria recommend use of CCTA for the intermediate risk category ${ }^{7,8}$ To assess the pre-test probability of having CAD prior to CCTA, clinical risk profiling is often used. Several algorithms to assess clinical risk have been developed, which predict for cardiovascular events in a different way, and are calibrated in different geographical regions and in diverse patient and population cohorts. Framingham risk score (FRS) predicts for 10-year risk of having any cardiovascular event, whereas PROCAM predicts for risk of myocardial infarction only. The SCORE algorithm predicts for death due to myocardial infarction and not for disease. Also, none of these scores were developed for use in symptomatic patients. The Diamond Forrester pre-test probability model was developed to assess probability of having significant CAD in symptomatic patients. ${ }^{9}$ Yet, it is not known which algorithm is most suitable to predict the likelihood of having CAD. The identification of the most precise algorithm to assess the likelihood of CAD is essential for effective alignment of healthcare resources and safety of the patient. We studied the most commonly used risk profiling algorithms in their ability to predict for 1) CAD on CCTA, and 2) for major adverse cardiovascular events, in patients presenting with chest pain at the cardiology outpatient clinic. 


\section{METHODS}

\section{Study population}

Between December 2007 and June 2010, 1,891 patients presenting with chest pain at our outpatient clinic were enrolled in this study. Inclusion criteria were a recent history of cardiac (a)typical chest pain, a diagnostic CCTA-scan, defined as seven or more interpretable coronary segments. Exclusion criteria were unstable angina, previous myocardial infarction, previous revascularization, hemodynamic instability, contrast allergy, pregnancy and renal failure. In 1,518 patients, a complete lipid spectrum was collected, Diamond Forrester pre-test probability, Framingham, PROCAM and SCORE risk score were assessed and a CCTA scan was made to assess the extent of CAD. In 222 subjects, CCTA was excluded because of artifact formation, or previous revascularization. Eventually, 1,296 subjects were analyzed in this study. Institutional Review Board and ethics committee at the Maastricht University Medical Center approved the study and all patients signed informed consent.

\section{Clinical definitions}

Cardiac risk factors were gathered at the outpatient clinic. Patients were classified as active smoker if they had smoked in the previous twelve weeks. Patients treated with a hypoglycemic agent or with a fasting plasma glucose $\geq 126$ $\mathrm{mg} / \mathrm{dL}$ were classified as diabetic. A family history of CAD was defined as having a first-degree relative with a history of myocardial infarction or sudden cardiac death before the age of sixty.

\section{Diamond Forrester score}

The probability of having significant CAD was calculated using the Diamond Forrester model. This model takes into account age, sex, and type of chest pain, which was classified as typical, atypical or non-anginal. ${ }^{9}$ The commonly used classification cut-offs of 30 and $70 \%$ were used..$^{10}$ Consequently, a score below $30 \%$ was considered low, $30-70 \%$ intermediate and $>70 \%$ high risk of having significant CAD.

\section{Framingham risk score}

The Framingham risk score is a multivariable risk function that predicts 10-year risk of developing cardiovascular disease events (coronary heart disease, stroke, 
peripheral artery disease or heart failure). The sex-specific scores incorporate age, total and high-density lipoprotein cholesterol, systolic blood pressure, treatment for hypertension, smoking and diabetic status. A score below $10 \%$ is considered low, $10-20 \%$ intermediate and $>20 \%$ high 10 -year risk of cardiovascular events. ${ }^{11}$

\section{PROCAM risk score}

PROCAM participants were followed up for acute coronary events (myocardial infarction, sudden cardiac death) for 10 years. The calibrated risk score included; age, LDL cholesterol, smoking, HDL cholesterol, systolic blood pressure, family history of premature myocardial infarction, diabetes mellitus and triglycerides. ${ }^{12}$ A score below $10 \%$ is considered low, $10-20 \%$ intermediate and $>20 \%$ high 10 -year risk of coronary events.

\section{SCORE risk score}

The SCORE predicts 10-year risk on fatal cardiovascular disease resulted in a model which included gender, age, systolic blood pressure, total cholesterol and smoking. A score of $0-4 \%$ was considered low, $5-9 \%$ intermediate and $\geq 10 \%$ high risk of cardiovascular death in 10 years. ${ }^{13}$

\section{CCTA acquisition}

CCTA was performed using a 64-slice CT scanner (Brilliance 64; Philips Healthcare) with a $64 \times 0.625 \mathrm{~mm}$ slice collimation, a gantry rotation time of $420 \mathrm{~ms}$ and a tube voltage of $80-120 \mathrm{kV}$ depending on the patient's height and weight. Patients received 5-20 mg of Metoprolol intravenously to lower the heart rate (HR) $<65 \mathrm{bpm}$ as well as sublingual nitroglycerin spray. HR and ECG were monitored during CCTA. CCTA was performed using $85-110 \mathrm{~mL}$ of contrast agent (Xenetix 350; Guerbet), which was injected in the antecubital vein at a rate of $6.0 \mathrm{~mL} / \mathrm{s}$, directly followed by $40 \mathrm{~mL}$ intravenous saline $(6.0$ $\mathrm{mL} / \mathrm{s}$ ). In patients with HR $<65 \mathrm{bpm}$, a prospective-gated "Step and shoot" protocol was used. ${ }^{14}$ In patients with HR $>65 \mathrm{bpm}$, a retrospective-gated "Helical" protocol with dose modulation was used to obtain the best image quality at minimal radiation dose. ${ }^{15}$ 


\section{CCTA coronary plaque assessment}

All CCTA-scans were independently analyzed by two experienced cardiologists, both blinded for patient details. In case of disagreement, consensus was reached by discussion. To indicate the location of coronary atherosclerosis, the classification of the American Heart Association (AHA) in 16 segments was used. ${ }^{16}$ The coronary artery tree was assessed using the source images on the Cardiac Comprehensive Analysis software (Philips Healthcare). Coronary plaques were defined as visible structures within or adjacent to the coronary artery lumen, clearly distinguished from the vessel lumen and the surrounding pericardial tissue. The degree of stenosis of atherosclerotic lesions was evaluated visually and classified as insignificant (no lesions, or one or more lesions with luminal stenosis of $<50 \%$ ), or significant (one or more lesions with luminal stenosis of $\geq 50) \cdot{ }^{17}$

\section{Statistics}

Data were analyzed using SPSS 17.0 (SPSS Inc). Continuous variables were reported as means and SDs and proportions (\%) were used for categorical values. Receiver operating curves (ROC) were produced and area under the curves (AUC) were reported for different risk scores in relation to significant CCTA lesions. Mc Nemar test was used to assess significance of difference between different risk score categories. All p-values were 2-sided, and a value below 0.05 was considered significant.

\section{Follow-up}

Electronic patient records were monitored for all-cause mortality and acute coronary syndrome (ACS), including myocardial infarction and unstable angina requiring hospitalization. Additionally, the national mortality records were checked. ACS was defined as typical angina pectoris and troponin T elevation $(>0.01 \mu \mathrm{g} / \mathrm{L})$ and ST-segment elevation/depression of $\geq 1 \mathrm{~mm}$, or at least two of these symptoms together with invasive angiographic conformation of a culprit lesion. ${ }^{18}$ Secondary endpoints included percutaneous coronary intervention (PCI) and coronary artery bypass graft (CABG) surgery. Revascularization procedures $<90$ days after CCTA were excluded as an event, as these would predominantly be CCTA driven. Further, we censored follow-up after the first endpoint, so that the recorded ACS was not a complication of revascularization 
therapy. Patients were seen by their cardiologist on a regular basis, and all visits were recorded in the electronic patient records.

\section{RESULTS}

\section{Patient characteristics}

Baseline characteristics are reported in table 1. Of 1,296 patients, any CAD was found in 806 (62\%), significant CAD in 317 (25\%). Mean follow-up period was $19 \pm 9$ months, and a total of 47 events were reported. Events consisted of 7 deaths, 18 cases of ACS (of which 5 were diagnosed as myocardial infarction) and 22 cases of revascularization (15 PCIs and 7 CABGs).

Table 1. Baseline characteristics

\begin{tabular}{ll}
\hline Age (years) & $56 \pm 11$ \\
Female gender & $606(46.8)$ \\
BMI $\left(\mathrm{kg} / \mathrm{m}^{2}\right)$ & $27 \pm 5$ \\
Active smoking & $316(24.4)$ \\
Diabetes mellitus & $102(7.9)$ \\
Positive family history & $522(40.3)$ \\
Systolic blood pressure (mmHg) & $142 \pm 19$ \\
Typical chest pain & $169(13)$ \\
Cholesterol (mg/dL): & \\
$\quad$ Total & $207 \pm 46$ \\
$\quad$ LDL & $128 \pm 42$ \\
$\quad$ HDL & $51 \pm 29$ \\
Triglycerides (mg/dL) & $153 \pm 100$ \\
Glucose (mg/dL) & $104 \pm 24$ \\
Creatinin (mg/dL) & $1.1 \pm 0.2$ \\
Clinical risk scores: & \\
$\quad$ Framingham & $21 \pm 16$ \\
PROCAM & $12 \pm 13$ \\
SCORE & $4 \pm 4$ \\
Diamond Forrester & $42 \pm 26$ \\
\hline
\end{tabular}

Continuous variables are described as mean $\pm \mathrm{SD}$, categorical variables as number (\%).

\section{Overall prediction of CAD per risk score}

In ROC-analysis for prediction of any coronary lesion, the areas under the curve for FRS; 0.74 (95\% confidence interval: 0.72 - 0.77) and for SCORE; 0.72 (95\% confidence interval: 0.70 - 0.75) were significantly higher than for PROCAM; 0.70 (95\% confidence interval: $0.67-0.73$; $\leq .03)$, which was significantly higher than for Diamond Forrester; 0.65 (95\% confidence interval: 0.62 - 
0.68; $\mathrm{p}<0.01$ ), figure $1 \mathrm{~A}$. Moreover, the areas under the curve for predicting significant CAD stenosis for FRS; 0.68 (95\% confidence interval: 0.64 - 0.72) and for SCORE; 0.69 (95\% confidence interval: 0.65 - 0.72) were significantly higher than for PROCAM; 0.64 (95\% confidence interval: 0.61 - 0.68 ; $\mathrm{p} \leq 0.001)$, as well as marginally higher than for Diamond Forrester; $0.65(95 \%$ confidence interval: $0.61-0.68 ; \mathrm{p}=0.05)$, figure $1 \mathrm{~B}$

Figure 1. ROC analysis

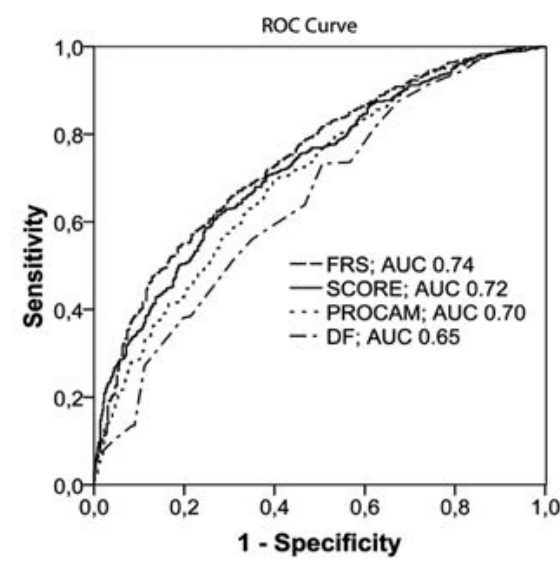

A. ROC analysis of Framingham, PROCAM, SCORE and Diamond Forrester score predicting any lesion on CCTA. Area under the curve is $0.74 ; 0.72 ; 0.70$ and 0.65 ; respectively.

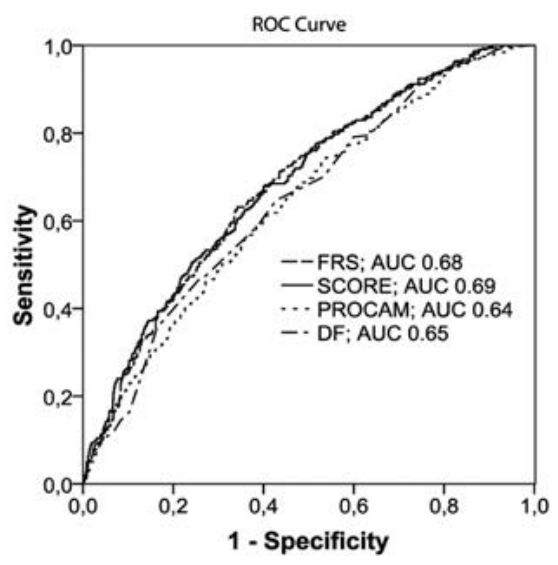

B. ROC analysis of Framingham, PROCAM, SCORE and Diamond Forrester score predicting $\geq 50 \%$ lesion on CCTA. Area under the curve is 0.68 ; $0.69 ; 0.64$ and 0.65 ; respectively.

\section{Risk categorization per risk score}

Table 2 displays the number (\%) of patients per risk category according to FRS, PROCAM, SCORE and Diamond Forrester in relation to CAD and number of events during follow-up. All scores were highly predictive for CAD $(p<0.001)$. Low FRS category showed the lowest number of patients with significant CAD (45/374=12\%), compared to patients with low risk using PROCAM, SCORE or Diamond Forrester $(\mathrm{p}<0.001)$. Also, low FRS category showed significantly lower number of events $(6 / 47=13 \%)$, compared to PROCAM and SCORE $(\mathrm{p}<0.001)$. When comparing to Diamond Forrester however, the number of events was not significantly lower $(\mathrm{p}=0.14)$. As a consequence, FRS high risk category included less patients with significant $\mathrm{CAD}$ and events, compared to patients with high risk using PROCAM, SCORE or Diamond Forrester 
$(\mathrm{p} \leq 0.02)$. In addition, in the low FRS category, $62 \%$ showed no CAD, compared to $48 \%, 47 \%$ and $47 \%$ for low PROCAM, SCORE and Diamond Forrester $(\mathrm{p} \leq 0.04)$. Figure 2 provides a visual overview of proportions of CAD in different risk categories, according to the different scoring models.

Figure 2. Proportions of significant, insignificant and no CAD for low-, intermediate- and highrisk categories by FRS, PROCAM, SCORE and Diamond Forrester (DF)

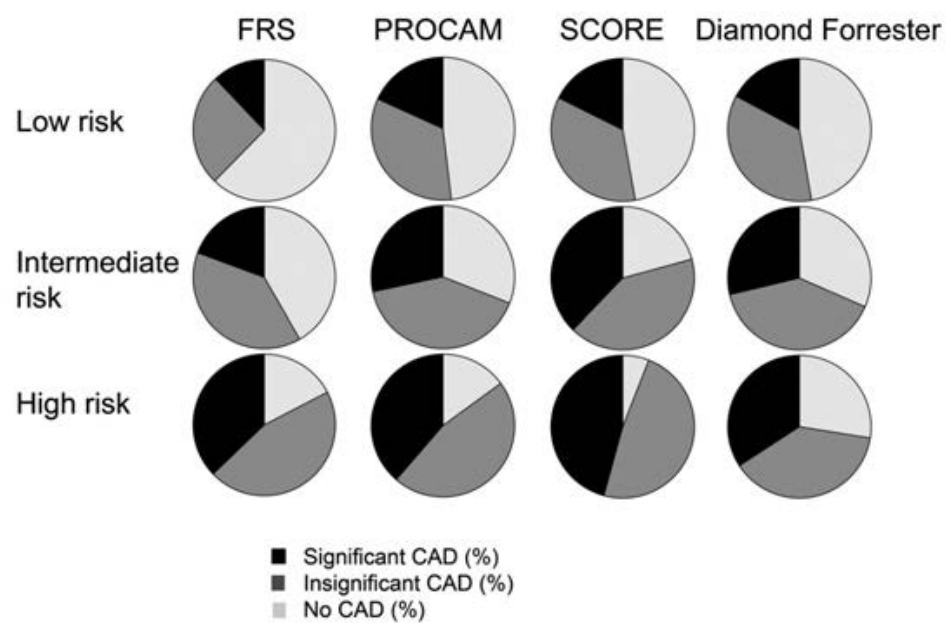




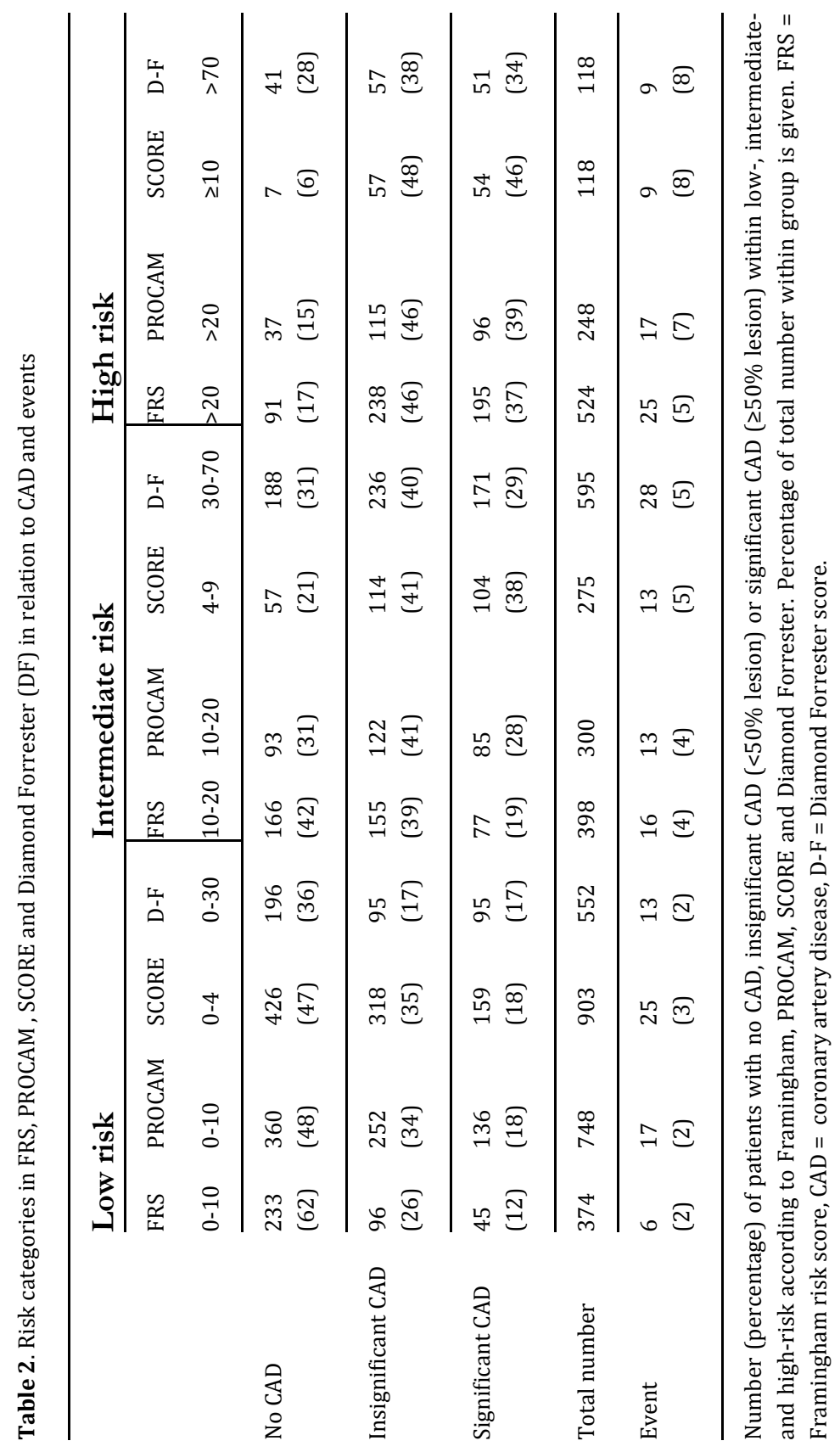




\section{DISCUSSION}

Our data show that the ability of FRS and SCORE to predict for CAD was similar and significantly better compared to PROCAM and marginally better than Diamond Forrester. The number of low risk patients showing significant CAD or events was lower using FRS, compared to PROCAM, SCORE and Diamond Forrester, using the indicated cut-off points for low-, intermediate- or high-risk for the different algorithms. Consequently, risk categorization using FRS is safest, but comes with a cost as more individuals with high risk do not have significant CAD or events, compared to PROCAM, SCORE and Diamond Forrester.

The cardiovascular disease epidemic produces a heavy burden on medical care. As the population ages, the cardiovascular disease burden will increase and costs are expected to rise substantially. ${ }^{19}$ Consequently, this of great concern for physicians confronted with patients presenting with stable chest pain. The prior question clinicians confronted with these patients want answered, is the chance of having CAD and secondly, the risk of having a cardiovascular event in the future. Clinical risk profiling remains the advised starting point for evaluation of these patients. Using the most effective clinical risk profiling algorithm is essential, and will make a substantial difference in risk stratification on a large scale. Above all, effective initial risk stratification will facilitate more efficient use of further diagnostics, all with their accompanying costs and disadvantages.

With the rapidly expanding epidemic of CAD in many developing countries, the identification of the most effective algorithm to predict for presence of CAD is an important step in allocation of appropriate care. Especially since in most developing countries the availability of diagnostics is limited and insurance programs are lacking. The fact that cost-effective use of health care resources also becomes more and more a critical issue in Western countries, makes it a global matter. An additional reason for the most precise prediction of presence of CAD is the issue of radiation dose in follow-up diagnostics. Concerns have been raised about radiation dose and cancer risk implied by cardiac imaging techniques. ${ }^{20}$ Therefore, although recent innovations in CT scanners have substantially lowered effective dose, CCTA in stable chest pain patients is still not generally advised. ${ }^{21}$ Most guidelines find CCTA appropriate in stable chest pain patients with intermediate risk profiles. ${ }^{7,8}$ In patients with a low risk profile, the benefits of CCTA do not seem to outweigh its costs and radiation dose. We showed that when applying these appropriateness criteria, 
FRS was safest, as less patients with any CAD, significant CAD or events were found in the low FRS group, as compared to the low PROCAM, SCORE group. Also, significantly less patients with any CAD or significant CAD were found in the low FRS group, as compared to the low Diamond Forrester group.

When using cardiovascular risk profiling algorithms to risk stratify stable chest pain patients prior to CCTA, one must realize that these scores were developed to predict coronary heart disease and not coronary atherosclerosis. However, there seems to be a strong relation between coronary atherosclerotic burden and risk of future coronary heart disease events. ${ }^{22}$ We acknowledge that all scores were based on different risk factors, and were calibrated on different geographical regions and populations. Above all, we investigated a symptomatic population. In contrast to the clinical risk algorithms, Diamond Forrester pretest probability score might be more appropriate for prediction of $\mathrm{CAD}$, but was never developed to predict for events. Surprisingly, the FRS, which was calibrated for an American population, performed better than the European calibrated PROCAM risk score. The major difference between the two algorithms is that FRS includes blood pressure treatment, further differences must be explained by calibration factors. For that matter, our findings seem concurrent with previous reports investigating FRS in the PROCAM population. Hense et al. reported FRS to overestimate actual risk in the PROCAM population. ${ }^{23}$ For the use of risk stratification prior to CCTA, this overestimation might result in more safety, as we reported the low FRS category to have less CAD and events in this study. In SCORE, no glucose or diabetic status is regarded in the risk score algorithm. Possibly, this may be a disadvantage in countries were DM is on the rise. In this study, the performance of Diamond Forrester seemed to somewhat disappoint. There are several explanations for this observation. First, the Diamond Forrester score was developed in the 1970s, for populations undergoing conventional angiography. In contrast, the current population undergoing CCTA is a relatively low risk group, in which the majority presented with atypical chest pain. Eventually, only $25 \%$ showed significant coronary stenosis. In addition, there is evidence that conventional angiography and CCTA seem to assess the severity of stenosis differently, especially in noncircular geometry. ${ }^{24}$ Furthermore, although symptoms are incorporated in the Diamond Forrester score, FRS includes more clinical risk factors. Given our data, this apparently compensates for the lack of information about symptoms. 
In patients categorized as low risk according to FRS, still $12 \%$ had significant lesions in our study. Also, 13\% of all events occurred in the low FRS group. This is in concurrence with previous reports, and questions the relative safety of using clinical risk profiling as a screening tool in patients with stable chest pain. ${ }^{25,26}$ Performance of calcium scoring in addition to clinical risk profiling has shown an increment in predictive value for CAD and cardiovascular events, and certainly makes a safer yet more expensive screening tool compared to clinical risk profiling only. ${ }^{27}$ There is ample evidence that calcium score is a good predictor for cardiovascular events. ${ }^{27,28}$ The role of CCTA as a risk predictor is still more uncertain, although CCTA is reported to have an excellent negative predictive value, ${ }^{6}$ as well as providing additive value in identifying the patient at risk. ${ }^{29}$ In addition, there is some evidence that CCTA might outperform clinical risk profiling as well as calcium scoring in predicting events. ${ }^{30}$ Question remains what will be the most cost-effective strategy on the long term. It is plausible that more extensive use of imaging techniques for early detection of patients at risk for CAD can increment preventive therapy. ${ }^{31}$ In the end, prevention of disease will be more cost-effective compared to treatment of manifest CAD, and innovations in imaging techniques such as CCTA will make its application more justifiable, probably even for low risk patients. However, for now the initial use of clinical risk profiling is a cornerstone in the assessment of patients to be assessed for CAD and determines the appropriateness of further CCTA studies. We hereby show that the use of FRS is safer to engage this role as compared to PROCAM, SCORE and Diamond Forrester score.

\section{Study limitations}

The analysis was strengthened by the relatively large sample size. In general, the study population consisted of patients of European descent, presenting with both typical and atypical stable chest pain, in The Netherlands. Therefore caution should be taken in interpreting these study results on other populations, and similar evaluations in different populations and regions worldwide seem valuable. We used: 1) clinical risk profiling algorithms that were developed to predict different cardiovascular events within a 10-year period and 2) Diamond Forrester score which was developed to predict pre-test probability of significant CAD prior to conventional angiography, to predict for CAD as assessed by CCTA. Although these scores were not developed for this purpose, there is no designated pre-test probability score for CCTA yet, resulting in the widespread alternative use of these scores. The relative short follow up time and 
small number of events made the data on cardiovascular events preliminary. We acknowledge the possibility that events could have presented elsewhere, resulting in an incomplete follow-up. However, as all patients were followed-up in our clinic, we expect the completeness of the follow-up to be high.

\section{Conclusion}

Our data show that in a stable chest pain population referred for CCTA, the ability of FRS and SCORE to predict for CAD was similar and significantly better compared to PROCAM and marginally better than Diamond Forrester. The number of low risk patients showing significant CAD or events was lower using FRS, compared to PROCAM, SCORE and Diamond Forrester, using the indicated cut-off points for low-, intermediate- or high-risk for the different risk profiling algorithms. Consequently, risk categorization using FRS seems to be safest in stable chest pain patients, but comes with a cost as more individuals with high risk will not have significant CAD or events, compared to PROCAM, SCORE and Diamond Forrester.

\section{References}

1. D'Agostino RB, Russell MW, Huse DM, Ellison RC, Silbershatz H, Wilson PW, Hartz SC. Primary and subsequent coronary risk appraisal: new results from the Framingham study. Am Heart J 2000;139:272-81.

2. Heidenreich PA, Trogdon JG, Khavjou OA, Butler J, Dracup K, Ezekowitz MD, Finkelstein EA, Hong Y, Johnston SC, Khera A, Lloyd-Jones DM, Nelson SA, Nichol G, Orenstein D, Wilson PW, Woo YJ. Forecasting the future of cardiovascular disease in the United States: a policy statement from the American Heart Association. Circulation 2000;123:933-44.

3. Patel V, Chatterji S, Chisholm D, Ebrahim S, Gopalakrishna G, Mathers C, Mohan V, Prabhakaran D, Ravindran RD, Reddy KS. Chronic diseases and injuries in India. Lancet 2011;377:413-28.

4. Executive Summary of The Third Report of The National Cholesterol Education Program (NCEP) Expert Panel on Detection, Evaluation, And Treatment of High Blood Cholesterol In Adults (Adult Treatment Panel III). JAMA 2001;285:2486-97. 
5. Gibbons RJ, Chatterjee K, Daley J, Douglas JS, Fihn SD, Gardin JM, Grunwald MA, Levy D, Lytle BW, O'Rourke RA, Schafer WP, Williams SV, Ritchie JL, Cheitlin MD, Eagle KA, Gardner TJ, Garson A, Jr., Russell RO, Ryan TJ, Smith SC, Jr. ACC/AHA/ACP-ASIM guidelines for the management of patients with chronic stable angina: a report of the American College of Cardiology/American Heart Association Task Force on Practice Guidelines (Committee on Management of Patients With Chronic Stable Angina). J Am Coll Cardiol 1999;33:2092-197.

6. Min JK, Shaw LJ, Devereux RB, Okin PM, Weinsaft JW, Russo DJ, Lippolis NJ, Berman DS, Callister TQ. Prognostic value of multidetector coronary computed tomographic angiography for prediction of all-cause mortality. J Am Coll Cardiol 2007;50:1161-70.

7. Mark DB, Berman DS, Budoff MJ, Carr JJ, Gerber TC, Hecht HS, Hlatky MA, Hodgson JM, Lauer MS, Miller JM, Morin RL, Mukherjee D, Poon M, Rubin GD, Schwartz RS. ACCF/ACR/AHA/NASCI/SAIP/SCAI/SCCT 2010 expert consensus document on coronary computed tomographic angiography: a report of the American College of Cardiology Foundation Task Force on Expert Consensus Documents. J Am Coll Cardiol 2010;55:2663-99.

8. Fox K, Garcia MA, Ardissino D, Buszman P, Camici PG, Crea F, Daly C, De Backer G, Hjemdahl P, Lopez-Sendon J, Marco J, Morais J, Pepper J, Sechtem U, Simoons M, Thygesen K, Priori SG, Blanc JJ, Budaj A, Camm J, Dean V, Deckers J, Dickstein K, Lekakis J, McGregor K, Metra M, Osterspey A, Tamargo J, Zamorano JL. Guidelines on the management of stable angina pectoris: executive summary: The Task Force on the Management of Stable Angina Pectoris of the European Society of Cardiology. Eur Heart J 2006;27:1341-81.

9. Diamond GA, Forrester JS. Analysis of probability as an aid in the clinical diagnosis of coronary-artery disease. N Engl J Med 1979;300:1350-8.

10. Meijboom WB, van Mieghem CA, Mollet NR, Pugliese F, Weustink AC, van Pelt N, Cademartiri F, Nieman K, Boersma E, de Jaegere P, Krestin GP, de Feyter PJ. 64-slice computed tomography coronary angiography in patients with high, intermediate, or low pretest probability of significant coronary artery disease. J Am Coll Cardiol 2007;50:146975.

11. D'Agostino RB, Sr., Vasan RS, Pencina MJ, Wolf PA, Cobain M, Massaro JM, Kannel WB. General cardiovascular risk profile for use in primary care: the Framingham Heart Study. Circulation 2008;117:743-53.

12. Assmann G, Cullen P, Schulte H. Simple scoring scheme for calculating the risk of acute coronary events based on the 10-year follow-up of the prospective cardiovascular Munster (PROCAM) study. Circulation 2002;105:310-5.

13. Conroy RM, Pyorala K, Fitzgerald AP, Sans S, Menotti A, De Backer G, De Bacquer D, Ducimetiere P, Jousilahti P, Keil U, Njolstad I, Oganov RG, Thomsen T, Tunstall-Pedoe H, Tverdal A, Wedel H, Whincup P, Wilhelmsen L, Graham IM. Estimation of ten-year risk of fatal cardiovascular disease in Europe: the SCORE project. Eur Heart J 2003;24:987-1003. 
14. Pontone G, Andreini D, Bartorelli AL, Cortinovis S, Mushtaq S, Bertella E, Annoni A, Formenti A, Nobili E, Trabattoni D, Montorsi P, Ballerini G, Agostoni P, Pepi M. Diagnostic accuracy of coronary computed tomography angiography: a comparison between prospective and retrospective electrocardiogram triggering. J Am Coll Cardiol 2009;54:346-55.

15. Hausleiter J, Meyer T, Hadamitzky M, Huber E, Zankl M, Martinoff S, Kastrati A, Schomig A. Radiation dose estimates from cardiac multislice computed tomography in daily practice: impact of different scanning protocols on effective dose estimates. Circulation 2006;113:1305-10.

16. Austen WG, Edwards JE, Frye RL, Gensini GG, Gott VL, Griffith LS, McGoon DC, Murphy ML, Roe BB. A reporting system on patients evaluated for coronary artery disease. Report of the Ad Hoc Committee for Grading of Coronary Artery Disease, Council on Cardiovascular Surgery, American Heart Association. Circulation 1975;51:5-40.

17. Raff GL, Abidov A, Achenbach S, Berman DS, Boxt LM, Budoff MJ, Cheng V, DeFrance T, Hellinger JC, Karlsberg RP. SCCT guidelines for the interpretation and reporting of coronary computed tomographic angiography. J Cardiovasc Comput Tomogr 2009;3:122-36.

18. Braunwald E, Antman EM, Beasley JW, Califf RM, Cheitlin MD, Hochman JS, Jones RH, Kereiakes D, Kupersmith J, Levin TN, Pepine CJ, Schaeffer JW, Smith EE, 3rd, Steward DE, Theroux P, Gibbons RJ, Alpert JS, Faxon DP, Fuster V, Gregoratos G, Hiratzka LF, Jacobs AK, Smith SC, Jr. ACC/AHA 2002 guideline update for the management of patients with unstable angina and non-ST-segment elevation myocardial infarction--summary article: a report of the American College of Cardiology/American Heart Association task force on practice guidelines (Committee on the Management of Patients With Unstable Angina). J Am Coll Cardiol 2002;40:1366-74.

19. Heidenreich PA, Trogdon JG, Khavjou OA, Butler J, Dracup K, Ezekowitz MD, Finkelstein EA, Hong Y, Johnston SC, Khera A, Lloyd-Jones DM, Nelson SA, Nichol G, Orenstein D, Wilson PW, Woo YJ. Forecasting the Future of Cardiovascular Disease in the United States: A Policy Statement From the American Heart Association. Circulation 2011;123:933-944.

20. Berrington de Gonzalez A, Mahesh M, Kim KP, Bhargavan M, Lewis R, Mettler F, Land C. Projected cancer risks from computed tomographic scans performed in the United States in 2007. Arch Intern Med 2009;169:2071-7.

21. Taylor AJ, Cerqueira M, Hodgson JM, Mark D, Min J, O'Gara P, Rubin GD, Kramer CM, Berman D, Brown A, Chaudhry FA, Cury RC, Desai MY, Einstein AJ, Gomes AS, Harrington R, Hoffmann U, Khare R, Lesser J, McGann C, Rosenberg A, Schwartz R, Shelton M, Smetana GW, Smith SC, Jr. ACCF/SCCT/ACR/AHA/ASE/ASNC/NASCI/SCAI/SCMR 2010 appropriate use criteria for cardiac computed tomography: a report of the American College of Cardiology Foundation Appropriate Use Criteria Task Force, the Society of Cardiovascular Computed Tomography, the American College of Radiology, the American Heart Association, the American Society of Echocardiography, the American Society of Nuclear Cardiology, the North American Society for Cardiovascular Imaging, the Society for Cardiovascular Angiography and Interventions, and the Society for Cardiovascular Magnetic Resonance. J Am Coll Cardiol 2010;56:1864-94. 
22. Hulten EA, Carbonaro S, Petrillo SP, Mitchell JD, Villines TC. Prognostic Value of Cardiac Computed Tomography Angiography A Systematic Review and Meta-Analysis. J Am Coll Cardiol 2011;57:1237-47.

23. Hense HW, Schulte H, Lowel H, Assmann G, Keil U. Framingham risk function overestimates risk of coronary heart disease in men and women from Germany--results from the MONICA Augsburg and the PROCAM cohorts. Eur Heart J 2003;24:937-45.

24. Arbab-Zadeh A, Texter J, Ostbye KM, Kitagawa K, Brinker J, George RT, Miller JM, Trost JC, Lange RA, Lima JA, Lardo AC. Quantification of lumen stenoses with known dimensions by conventional angiography and computed tomography: implications of using conventional angiography as gold standard. Heart 2010;96:1358-63.

25. Nair D, Carrigan TP, Curtin RJ, Popovic ZB, Kuzmiak S, Schoenhagen P, Flamm SD, Desai MY. Association of coronary atherosclerosis detected by multislice computed tomography and traditional risk-factor assessment. Am J Cardiol 2008;102:316-20.

26. Nucifora G, Schuijf JD, van Werkhoven JM, Jukema JW, Djaberi R, Scholte AJ, de Roos A, Schalij MJ, van der Wall EE, Bax JJ. Prevalence of coronary artery disease across the Framingham risk categories: coronary artery calcium scoring and MSCT coronary angiography. J Nucl Cardiol 2009;16:368-75.

27. Sarwar A, Shaw LJ, Shapiro MD, Blankstein R, Hoffmann U, Cury RC, Abbara S, Brady TJ, Budoff MJ, Blumenthal RS, Nasir K. Diagnostic and prognostic value of absence of coronary artery calcification. JACC Cardiovasc Imaging 2009;2:675-88.

28. Erbel R, Mohlenkamp S, Moebus S, Schmermund A, Lehmann N, Stang A, Dragano N, Gronemeyer D, Seibel R, Kalsch H, Brocker-Preuss M, Mann K, Siegrist J, Jockel KH. Coronary risk stratification, discrimination, and reclassification improvement based on quantification of subclinical coronary atherosclerosis: the Heinz Nixdorf Recall study. J Am Coll Cardiol 2010;56:1397-406.

29. Motoyama S, Sarai M, Harigaya H, Anno H, Inoue K, Hara T, Naruse H, Ishii J, Hishida H, Wong ND, Virmani R, Kondo T, Ozaki Y, Narula J. Computed tomographic angiography characteristics of atherosclerotic plaques subsequently resulting in acute coronary syndrome. J Am Coll Cardiol 2009;54:49-57.

30. Hadamitzky M, Distler R, Meyer T, Hein F, Kastrati A, Martinoff S, Schomig A, Hausleiter J. Prognostic value of coronary computed tomographic angiography in comparison with calcium scoring and clinical risk scores. Circ Cardiovasc Imaging 2011;4:16-23.

31. Fuster V, Vahl TP. The role of noninvasive imaging in promoting cardiovascular health. J Nucl Cardiol 2010;17:781-90. 



\title{
Chapter
}

\section{Epicardial Adipose Tissue Volume as a Predictor for Coronary Artery Disease in Diabetic, Impaired Fasting Glucose and Non-Diabetic Patients presenting with Chest Pain}

\author{
Mathijs O. Versteylen, Richard A. Takx, Ivo A. Joosen, \\ Patricia J. Nelemans, Marco Das, Harry J. Crijns, Leonard \\ Hofstra, Tim Leiner
}

Eur Heart J Cardiovasc Imaging. 2012;13:517-23. 


\begin{abstract}
Aims Epicardial adipose tissue (EAT) volume has been associated with coronary artery disease (CAD). As diabetes mellitus type 2 (DM2) patients have higher EAT volumes, it has been suggested that EAT may play a role in promoting CAD in these patients. Aim of this study was to examine the association between EAT and CAD in DM2, impaired fasting glucose (IFG) and control patients, presenting with stable chest pain.

Methods 410 stable chest pain patients underwent multidetector cardiac CT angiography (CCTA) to assess presence of CAD. The extent of CAD was expressed as the number of affected segments. EAT volume was measured using 3-dimensial volumetric quantification. EAT was compared using ANOVA, logistic and linear regression models were used to assess its predictive value. Multivariable regression analysis corrected for traditional risk factors was performed.
\end{abstract}

Results Eighty-three patients had DM2, 118 IFG and there were 209 controls. DM2 as well as IFG patients had higher EAT volumes compared to controls $\left(98 \pm 41,92 \pm 39\right.$ and $75 \pm 34 \mathrm{~cm}^{3}$, respectively; $\left.\mathrm{p}<0.001\right)$. EAT predicted the presence (OR 1.01; $\mathrm{p}<0.001$ ) and extent of CAD (B 0.01; $\mathrm{p}<0.001$ ). The associations were equal in all subgroups. However, in a multivariable regression model corrected for traditional cardiovascular risk factors, EAT was not an independent predictor for the presence or extent of CAD (OR 1.00; $p=0.88$ and $B-0.11$; $\mathrm{p}=0.68$, respectively).

Conclusion EAT volume is associated with CAD in DM2, IFG and control patients. However, EAT is not an independent predictor for CAD in patients presenting with stable chest pain. 


\section{INTRODUCTION}

Epicardial adipose tissue (EAT) has been proposed as a risk factor for coronary artery disease (CAD), ${ }^{1-3}$ as well as a predictor for the development of future cardiovascular events. ${ }^{4-6}$ Located between the myocardium and the visceral pericardium, EAT is visceral fat that directly surrounds the coronary arteries. It may work as an endocrine organ secreting hormones, bioactive adipocytokines and chemokines, ${ }^{7,8}$ in this way potentially promoting CAD. ${ }^{1}$ Consequently, EAT may have a potential role as a parameter for risk stratification purposes in patients presenting with chest pain. For instance, in patients undergoing computed tomography (CT) calcium scoring, EAT could be easily assessed on the non-enhanced CT image, in this way potentially adding value to this diagnostic test.

Patients with diabetes mellitus type 2 (DM2) and impaired fasting glucose (IFG) suffer from a 2-4 fold higher cardiovascular mortality compared to normoglycaemic patients. ${ }^{9,10}$ Also, patients with DM2 show a markedly increased prevalence of CAD. ${ }^{11,12}$ As both IFG and DM2 patients are reported to have higher EAT volumes, ${ }^{13,14}$ it is of particular interest to investigate the predictive value of $\mathrm{EAT}$ as a risk factor for $\mathrm{CAD}$ in these two patient categories.

Therefore, the purpose of the present study is to examine the relation between EAT and CAD in DM2, IFG and control patients with stable chest pain. Subsequently, we sought to establish whether EAT measurement offers additional value in predicting risk of CAD beyond traditional cardiovascular risk factors in this patient category.

\section{METHODS}

\section{Study population}

From December 2007 until June 2010, we prospectively included stable chest patients referred for coronary CT-angiography (CCTA) to rule out CAD. Included were patients with a recent history of atypical or typical chest pain, in which fasting serum was collected. Excluded were patients with unstable angina, hemodynamic instability, prior history of CAD, pregnancy, renal insufficiency (defined as serum creatinine $>110 \mathrm{mmol} / \mathrm{L}$ ), iodine allergy or nondiagnostic CCTA. From 934 included patients, 78 had a non-diagnostic scan. Within the remaining study population, 83 patients had DM2 and 118 patients had IFG. From the remaining 655 patients, 209 randomly selected patients with 
lation studied consisted of 410 patients. Institutional Review Board and ethics committee approved the study and all patients signed informed consent prior to inclusion in the study.

\section{Risk factor assessment}

Clinical risk factors were prospectively assessed. Patients were defined as having DM2 according to the expert committee on the diagnosis and classification of diabetes mellitus. ${ }^{15}$ IFG subjects were defined as those with fasting plasma glucose levels between $6.1 \mathrm{mmol} / \mathrm{L}$ and $6.9 \mathrm{mmol} / \mathrm{L} .15,16$ Control subjects were defined as those with fasting plasma glucose levels lower than $6.1 \mathrm{mmol} / \mathrm{L}$ and no family history of diabetes mellitus. Smoking was defined as current smoking. A positive family history was defined as having a first degree relative with a history of myocardial infarction or sudden cardiac death before the age of 60 . Next to fasting glucose, total cholesterol, HDL, triglycerides, concentrations were measured using the Synchron LX20 (Beckman Coulter Inc., Brea, CA, USA). Low-density lipoprotein (LDL) was calculated using the Friedewald equation, except for subjects with triglycerides $>4.5 \mathrm{mmol} / \mathrm{L}$ or total cholesterol $<1.3 \mathrm{mmol} / \mathrm{L}$, in which case LDL was determined on the Cobas Mira Plus (Roche Diagnostics, Basel, Switzerland).

\section{CCTA acquisition}

Scans were performed using a 64-slice multidetector-row CT-scanner (Brilliance 64, Philips Healthcare, Best, The Netherlands) with a $64 \times 0.625 \mathrm{~mm}$ slice collimation, a gantry rotation time of $420 \mathrm{~ms}$ and a tube voltage of $120 \mathrm{kV}$. A non-enhanced scan was performed to determine the calcium score using the Agatston method. ${ }^{17}$ This was followed by CCTA using 85 to $110 \mathrm{~mL}$ of contrast agent (Xenetix 350, Guerbet, Roissy CdG Cedex, France), which was injected in the antecubital vein at $6.0 \mathrm{~mL} / \mathrm{s}$, directly followed by $40 \mathrm{~mL}$ of intravenous saline at $6.0 \mathrm{~mL} / \mathrm{s}$ using a dual-head power injector. In patients with a heart rate $<65 \mathrm{bpm}$, a prospectively-gated axial scan protocol was used, and in those with a heart rate $>65 \mathrm{bpm}$, a retrospectively-gated "Helical" protocol with dose modulation to obtain the best image quality at minimal radiation dose was performed. ${ }^{18,19}$ Tube current varied from 150-210 mAs and from 600-1000 $\mathrm{mAs}$, for "prospectively-gated axial" and "Helical" protocols, respectively, depending on patients weight and height. Patients received 5-20 mg of Metoprolol (AstraZeneca, Zoetermeer, the Netherlands) intravenously to lower the 
heart rate $<65 \mathrm{bpm}$ as well as $0.8 \mathrm{mg}$ sublingual nitroglycerin spray (PohlBoskamp, Hohenlockstedt, Germany).

\section{Epicardial adipose tissue assessment}

EAT volume was retrospectively measured using dedicated volumetric software (Volume, Leonardo, Siemens Healthcare, Erlangen, Germany). The most cranial slice taken into account was at the level of the carina and the caudal slice limit was identified as the last slice containing any portion of the heart. Within this area, we manually traced the pericardium every $1 \mathrm{~cm}$, using the region of interest tool, to separate epicardial fat from pericardial fat. The software semiautomatically reconstructed the pericardium into a $3 \mathrm{D}$ region of interest, which was controlled visually and could be adjusted manually. Within this region of interest defined by the pericardium, contiguous 3D voxels between -190 and 30 Hounsfield Units were defined as fat voxels. ${ }^{20}$ This resulted in a measurement of EAT in $\mathrm{cm}^{3}$. A prior study reported that threshold-based 3dimensional (3D) volumetric quantification of EAT has a good correlation $(\mathrm{r}=0.956, \mathrm{p}<0.001)$ with the $2 \mathrm{D}$ short-axis based model and is less time consuming. ${ }^{21}$ To determine reproducibility of EAT measurements, EAT volume was independently measured a second time by MV and RT, blinded from patient characteristics, in a subset of 20 patients.

\section{Coronary assessment}

Using the American Heart Association (AHA) 16 coronary segments model,22 scans were independently analyzed by an experienced cardiologist and a radiologist who were blinded for clinical information. The coronary artery tree was assessed using the source images on the provided software (Brilliance Workplace Portal v2.6.0.27, Philips Healthcare, Best, The Netherlands), and the degree of stenosis was visually defined as mild diameter stenosis $(<50 \%)$, moderate $(50-70 \%)$ and severe diameter stenosis $(>70 \%)$. Significant lesions were considered to be moderate or severe (one or more lesions with diameter stenosis $\geq 50 \%$ ). ${ }^{23}$ An involvement score was calculated by counting all diseased vessel segments (irrespective of degree of stenosis), and the total score ranged from 0-16.24 Plaques were categorized as calcified (exclusively content with density $>130$ Hounsfield Units), non-calcified (exclusively content with density $<130$ Hounsfield Units), or mixed (characteristics of both calcified and noncalcified plaques). 


\section{Coronary artery disease outcome measures}

Three different outcome measures for CAD were investigated.

1) Presence of any CAD; which was classified as a binary variable regarded positive if there was any plaque detected by CCTA, irrespective of stenosis or plaque morphology.

2) Presence of significant $\mathrm{CAD}$; which was also a binary variable regarded positive in case of one or more coronary artery stenosis of at least 50\% (as determined visually on CCTA).

3) Extent of CAD; measured by the previously described involvement score, i.e. the number of affected segments as determined by CCTA, again irrespective of stenosis severity or plaque morphology. Also measured by calcium score; this method was added to provide an additional measurement for extent of CAD.

\section{Statistical analysis}

Data were analyzed using SPSS version 17.0 (SPSS Inc., Chicago, IL, USA). Continuous variables were reported as means \pm standard deviation (SD) and proportions (\%) were used for categorical values. Reproducibility was determined by calculating intraclass correlation coefficients (ICC), using a two-way mixed effects model, testing for absolute agreement. To assess differences between groups, ANOVA was used for continuous variables and chi-square test for categorical variables. Univariable and multivariable analyses were performed using linear regression for continuous outcomes or binary logistic regression for dichotomous outcomes. All variables considered to be clinically relevant risk factors were entered into the multivariable models. Regression coefficients from multivariable analysis estimated the independent effect of variables entered into the model. 95\% confidence intervals (CI) were used to evaluate whether the effects were statistically significant. Two-tailed P-values $<0.05$ were used to indicate statistical significance.

\section{RESULTS}

\section{Study population}

Mean age $( \pm S D)$ was $57 \pm 11$ years and $202(49 \%)$ were male. Median EAT volume was $81 \mathrm{~cm}^{3}$. There were $262(64 \%)$ patients with any CAD, of which 93 had a significant $(\geq 50 \%)$ coronary stenosis. Baseline characteristics are present- 
ed in table 1. When the DM2 and IFG group were compared to controls, they tended to be older, had a higher BMI, higher systolic blood pressure and lower serum cholesterol values (which was due to more statin use). In addition, EAT volumes, calcium scores and involvement scores were higher, table 1.

Table 1. Baseline characteristics for total population and per glycaemic subgroup

\begin{tabular}{|c|c|c|c|c|c|}
\hline & $\begin{array}{c}\text { Total } \\
(n=410)\end{array}$ & $\begin{array}{c}\text { DM2 } \\
(n=83)\end{array}$ & $\begin{array}{c}\text { IFG } \\
(n=118)\end{array}$ & $\begin{array}{l}\text { Controls } \\
(n=209)\end{array}$ & P-value \\
\hline Age (years) & $57 \pm 11$ & $60 \pm 10$ & $58 \pm 12$ & $55 \pm 11$ & 0.001 \\
\hline Male gender & $202(49)$ & $34(45)$ & 69 (59) & $96(46)$ & 0.06 \\
\hline $\operatorname{BMI}\left(\mathrm{kg} / \mathrm{m}^{2}\right)$ & $27 \pm 4$ & $29 \pm 5$ & $27 \pm 4$ & $26 \pm 4$ & $<0.001$ \\
\hline Active smoking & $101(25)$ & $21(25)$ & $26(22)$ & $54(26)$ & 0.74 \\
\hline Positive family history & $157(38)$ & $30(36)$ & $45(38)$ & $82(39)$ & 0.89 \\
\hline Systolic blood pressure(mmHg) & $144 \pm 19$ & $149 \pm 20$ & $145 \pm 20$ & $141 \pm 17$ & 0.007 \\
\hline \multicolumn{6}{|l|}{ Cholesterol (mmol/L) } \\
\hline Total & $5.3 \pm 1.2$ & $4.6 \pm 1.2$ & $5.4 \pm 1.1$ & $5.5 \pm 1.1$ & $<0.001$ \\
\hline LDL & $3.2 \pm 1.1$ & $2.6 \pm 1.0$ & $3.4 \pm 1.1$ & $3.4 \pm 1.1$ & $<0.001$ \\
\hline HDL & $1.3 \pm 0.5$ & $1.2 \pm 0.5$ & $1.2 \pm 0.3$ & $1.4 \pm 0.6$ & 0.002 \\
\hline Triglycerides (mmol/L) & $1.8 \pm 1.2$ & $1.8 \pm 1.2$ & $1.9 \pm 1.4$ & $1.7 \pm 0.9$ & 0.13 \\
\hline Statin use & $172(42)$ & $61(74)$ & $40(34)$ & $71(34)$ & $<0.001$ \\
\hline EAT volume $\left(\mathrm{cm}^{3}\right)$ & $84 \pm 38$ & $98 \pm 41$ & $92 \pm 39$ & $75 \pm 34$ & $<0.001$ \\
\hline Calcium score & $94 \pm 175$ & $170 \pm 239$ & $88 \pm 156$ & $67 \pm 143$ & $<0.001$ \\
\hline Involvement score & $2.2 \pm 2.5$ & $3.0 \pm 2.8$ & $2.3 \pm 2.7$ & $1.8 \pm 2.2$ & $<0.001$ \\
\hline Significant coronary lesion & $93(23)$ & $22(27)$ & $28(24)$ & $43(21)$ & 0.52 \\
\hline
\end{tabular}

Continuous variables are described as mean \pm SD, categorical variables as number (\%). BMI; Body Mass Index, LDL; low density lipoprotein, HDL; high density lipoprotein, DM2; diabetes mellitus type 2, IFG; impaired fasting glucose, EAT; epicardial adipose tissue.

\section{Reproducibility}

The reproducibility of the 3-D volumetric EAT quantification technique was excellent. The intra-observer variability was low; ICC $0.93 ; \mathrm{p}<0.001$ and the inter-observer agreement was good, ICC $0.90 ; \mathrm{p}<0.001$.

\section{Glycaemic state and EAT volume}

Patients with DM2 as well as patients with IFG both had significantly higher EAT volumes when compared to controls, figure 1. The mean EAT volume of DM2 and IFG subjects, $98 \pm 41 \mathrm{~cm}^{3}$ and $92 \pm 39 \mathrm{~cm}^{3}$, respectively, was markedly higher than $75 \pm 34 \mathrm{~cm}^{3}$ in controls, $(\mathrm{p}<0.001)$. This association was independent from traditional cardiovascular risk factors when corrected for age, gender, 
BMI, systolic blood pressure, positive family history and total cholesterol $(\mathrm{p}=0.04)$.

Figure 1. Mean EAT volume for controls, IFG subjects and patients with DM2

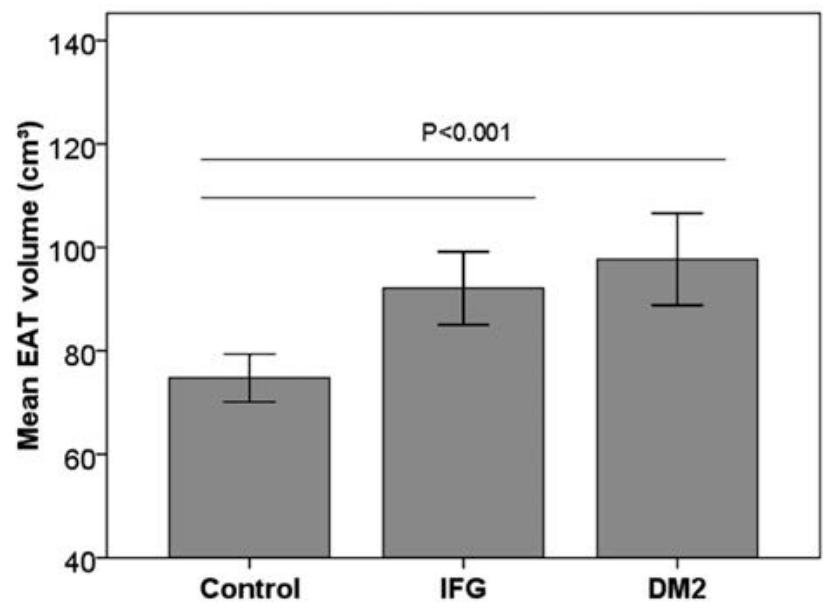

DM2 as well as IFG patients had higher EAT volumes compared to controls $(98 \pm 41,92 \pm 39$ and $75 \pm 34 \mathrm{~cm}^{3}$, respectively; $\mathrm{p}<0.001$ ).

\section{EAT as a predictor of CAD}

Univariable analysis showed that EAT volume predicted for the presence of any plaque on CCTA, but not for the presence of significant plaques (OR 1.01, $\mathrm{p}<0.001$ and OR $1.00, \mathrm{p}=0.15$, respectively). Table 2 displays the association between EAT and presence of CAD for all patients, as well as for glycaemic subgroups. Also, EAT was significantly associated with the extent of CAD. Linear regression analysis showed that for each increase of $10 \mathrm{~cm}^{3}$ EAT the Agatston calcium score increased 6 units, and the involvement score increased with 0.13 segments $(\mathrm{P}=0.009$ and $\mathrm{P}<0.001$, respectively). In addition, the association between EAT and extent of CAD (involvement score) was investigated per glycaemic subgroup (table 3). 
Table 2. EAT as predictor for the presence of any CAD, for all patients and subgroups

\begin{tabular}{lccc} 
& OR & $95 \%$ CI & P-value \\
\hline All patients $(n=410)$ & 1.01 & $1.01-1.02$ & $<0.001$ \\
Controls $(n=209)$ & 1.01 & $1.00-1.02$ & 0.01 \\
IFG (n=118) & 1.01 & $1.00-1.02$ & 0.05 \\
DM2 (n=83) & 1.02 & $1.00-1.03$ & 0.04 \\
\hline
\end{tabular}

EAT means epicardial adipose tissue volume $\left(\mathrm{cm}^{3}\right)$, CAD means coronary artery disease, IFG means impaired fasting glucose, DM2 means diabetes mellitus type 2.

Table 3. EAT as predictor for the extent of CAD, for all patients and per subgroup

$$
\text { B-value } \quad 95 \% \mathrm{CI} \quad \text { P-value }
$$

\begin{tabular}{lccc}
\hline All patients $(\mathrm{n}=410)$ & 0.013 & $0.007-0.019$ & $<0.001$ \\
Controls $(\mathrm{n}=209)$ & 0.012 & $0.004-0.021$ & 0.006 \\
IFG $(\mathrm{n}=118)$ & 0.011 & $-0.001-0.024$ & 0.07 \\
DM2 $(\mathrm{n}=83)$ & 0.007 & $-0.008-0.022$ & 0.35 \\
\hline
\end{tabular}

EAT means epicardial adipose tissue volume $\left(\mathrm{cm}^{3}\right)$, CAD means coronary artery disease, IFG means impaired fasting glucose, DM2 means diabetes mellitus type 2 .

\section{Addition of EAT to glycaemic state}

Next to EAT, both DM2 as well as IFG were associated with the presence of $\mathrm{CAD}$ as well as the extent of CAD as determined by involvement score (OR 1.7, $\mathrm{p}=0.001$ and $\mathrm{B} 0.65, \mathrm{p}<0.001$ for $\mathrm{DM} 2$, and $\mathrm{OR} 1.5, \mathrm{p}=0.11$ and $\mathrm{B}=0.54$, $\mathrm{p}=0.05$ for IFG). Interestingly, the number of patients with any CAD increased in the diabetic group if they also had a high EAT volume. Although not significant, $70 \%$ of diabetic patients with low EAT volumes $\left(<80 \mathrm{~cm}^{3}\right)$ had evidence of at least some CAD; this fraction increased to $82 \%$ when EAT volume was high $\left(\geq 80 \mathrm{~cm}^{3} ; \mathrm{p}=0.22\right)$. For patients with IFG, these numbers were $65 \%$ and $67 \%(\mathrm{p}=0.77)$. In the control group, $53 \%$ of patients with low EAT volumes had evidence of at least some CAD; this fraction increased to 63\% when EAT volume was high $(\mathrm{p}=0.13)$. When measuring extent of $\mathrm{CAD}$, a similar pattern was seen. For instance, in the control group the involvement score increased from 1.5 \pm 1.9 for low EAT volumes to 2.2 \pm 2.4 for high EAT volumes, $\mathrm{p}=0.04$. Overall, the additive value of EAT volume over diabetic status for the prediction of CAD was low. The association between DM2 and extent of CAD seemed stronger than between EAT and CAD, as is graphically shown in figure 2 . 


\section{Multivariate analysis}

As expected, the strongest independent predictors for the presence of CAD were traditional risk factors, such as age, male gender, smoking and systolic blood pressure (table 4). Also, DM2 was an independent predictor for the extent of CAD, while EAT did not independently predict for the presence or extent of CAD (as determined by involvement score, table 5).

Figure 2. Relation of both EAT and glycaemic status with extent of CAD
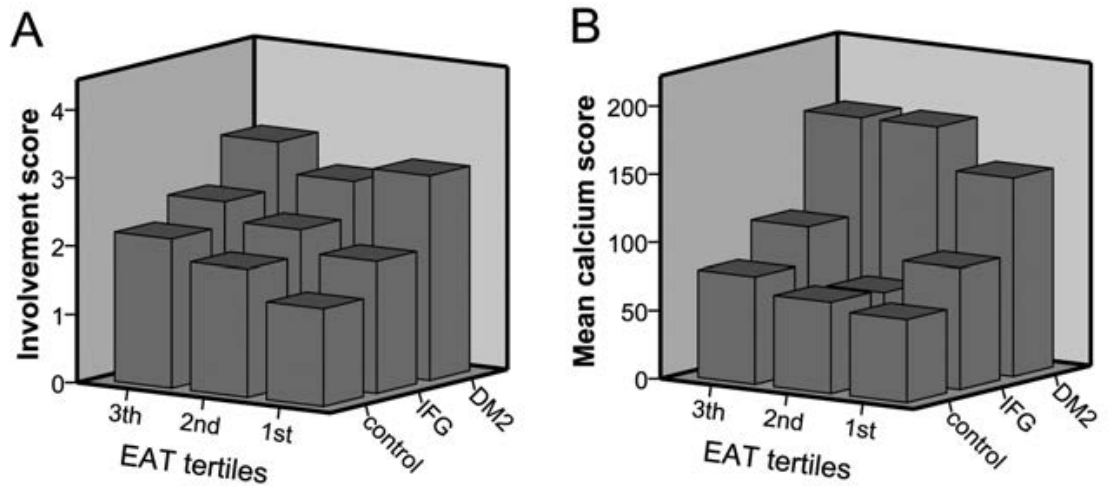

Relation of both EAT and glycaemic status with extent of CAD, measured as; a) calcium score (Agatston score) and b) involvement score (number of coronary segments showing CAD).

\section{Plaque morphology}

When investigating patients with at least one coronary plaque, we found DM2 patients to have a higher number of calcified plaques when compared to control patients $(2.4 \pm 2.2$ vs. $1.8 \pm 1.7$, respectively, $\mathrm{p}=0.03)$. Concerning EAT, no differences in number of calcified, mixed or non-calcified plaques were found between EAT volume tertiles (figure 3).

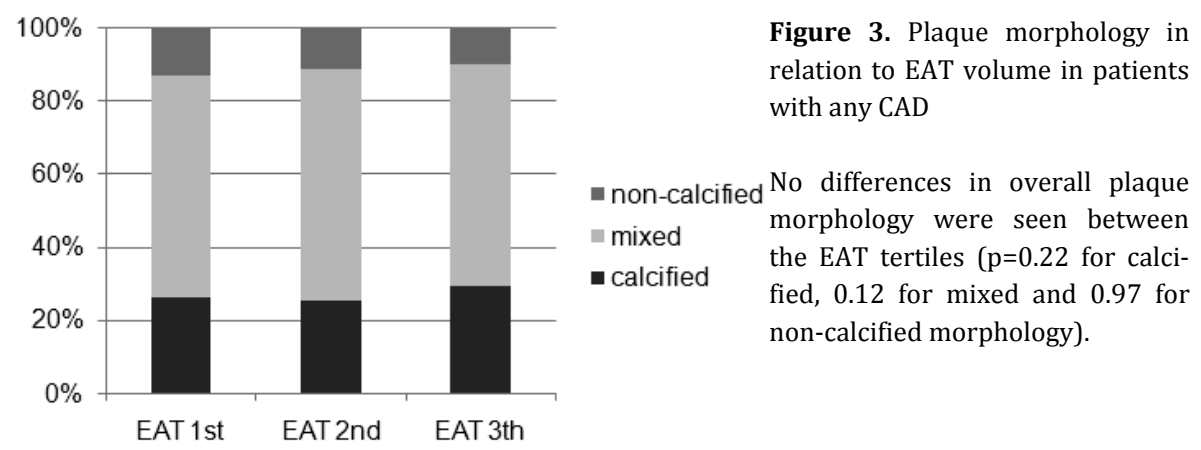


Table 4. Multivariable binary regression model predicting for presence of any CAD

\begin{tabular}{lccc} 
& OR & $95 \%$ CI & P-value \\
\hline Age & 1.08 & $1.05-1.11$ & $<0.001$ \\
Male gender & 2.45 & $1.47-4.10$ & 0.001 \\
Active smoking & 2.23 & $1.26-3.97$ & 0.006 \\
Systolic BP (mmHg) & 1.01 & $1.00-1.02$ & 0.19 \\
Positive family history & 0.91 & $0.57-1.47$ & 0.71 \\
Total cholesterol & 0.95 & $0.77-1.18$ & 0.64 \\
BMI $\left(\mathrm{kg} / \mathrm{m}^{2}\right)$ & 1.05 & $0.98-1.12$ & 0.16 \\
DM2 & 1.89 & $0.94-3.80$ & 0.08 \\
IFG & 1.03 & $0.60-1.77$ & 0.99 \\
EAT $\left(\mathrm{cm}^{3}\right)$ & 1.00 & $0.99-1.01$ & 0.88 \\
\hline
\end{tabular}

$\mathrm{CAD}=$ coronary artery disease, $\mathrm{BP}=$ blood pressure, $\mathrm{BMI}=$ body mass index, $\mathrm{DM} 2=$ diabetes mellitus type 2, IFG=impaired fasting glucose, EAT=epicardial adipose tissue volume $\left(\mathrm{cm}^{3}\right)$.

Table 5. Multivariable linear regression model predicting for extent of CAD (involvement score)

\begin{tabular}{lccc} 
& B-value & $95 \%$ CI & P-value \\
\hline Age & 0.06 & $0.04-0.08$ & $<0.001$ \\
Male gender & 1.00 & $0.51-1.48$ & $<0.001$ \\
Active smoking & 0.89 & $0.35-1.44$ & 0.001 \\
Systolic BP (mmHg) & 0.02 & $0.00-0.03$ & 0.012 \\
Positive family history & -0.06 & $-0.54-0.42$ & 0.81 \\
Total cholesterol & -0.18 & $-0.39-0.03$ & 0.10 \\
BMI $\left(\mathrm{kg} / \mathrm{m}^{2}\right)$ & -0.01 & $-0.07-0.06$ & 0.87 \\
DM2 & 0.83 & $0.17-1.48$ & 0.01 \\
IFG & 0.19 & $-0.35-0.73$ & 0.50 \\
EAT $\left(\mathrm{cm}^{3}\right)$ & 0.00 & $-0.01-0.01$ & 0.87 \\
\hline
\end{tabular}

$\mathrm{CAD}=$ coronary artery disease, $\mathrm{BP}=$ blood pressure, $\mathrm{BMI}=$ body mass index, $\mathrm{DM} 2=$ diabetes mellitus type 2, IFG=impaired fasting glucose, EAT=epicardial adipose tissue volume $\left(\mathrm{cm}^{3}\right)$.

\section{DISCUSSION}

In the current clinical study we investigated the association between EAT, glycaemic state and CAD in stable chest pain patients. First, we found that patients with DM2 and IFG showed significantly higher EAT volumes, independent from traditional risk factors including BMI. This finding is partly concurrent with previous research. ${ }^{13,14,25}$ Second, we observed a clear association between EAT and the presence and extent of CAD in the total patient group. The magnitude of this association did not differ between glycaemic subgroups. However, EAT was not an independent predictor of CAD, while DM2 as well as other traditional risk factors were. 
So far, there is still debate about the role of EAT as a cardiovascular risk factor. Reports suggesting EAT to be an independent risk factor for cardiovascular events, are a large case-control study, ${ }^{5}$ and a study using age and sexmatched control groups. ${ }^{6}$ On the other hand, several studies in which no independent association was found were published.,14,26 Regarding the association with CAD, our study design reflects a real world clinical setting physicians find themselves confronted with, and provides relevant information about the value of different clinical risk factors in this patient category. Our data suggests that traditional risk factors, and in particular diabetic state outweigh EAT as a risk factor for CAD. This observation is further supported by the strong correlation between glycaemic state and presence or extent of CAD. In perspective, a study by Graves et al. demonstrated the important correlation between glucose serum levels and the gravity of prognosis, even in non-diabetics. ${ }^{27}$

Although we found EAT volume to be associated with both the presence and extent of CAD, no relation between EAT volume and the occurrence of stenotic coronary lesions could be demonstrated, which is in line with previous findings. ${ }^{28}$ In literature, the association between EAT and calcium score was described before. ${ }^{4}$ Calcium score is a generally accepted technique to measure extent of $\mathrm{CAD}$, and is known to confer important prognostic information. ${ }^{29} \mathrm{~A}$ potential limitation of this technique is that it neglects the presence of noncalcified lesions. To overcome this, we reported the number of atherosclerotic segments involved in the total coronary tree as assessed by CCTA, resulting in a comprehensive scoring of CAD extent.

Interestingly, EAT volume was not associated with a specific type of plaque morphology in this study. Some morphologic characteristics have been used to identify patients at higher risk for adverse cardiac events. ${ }^{30}$ Although we investigated a larger population compared to most previous studies, our findings do not support previous observations that patients with high EAT volumes show more non-calcified or mixed plaques. ${ }^{31,32}$ Patients with DM2 status did show a higher amount of calcified plaques, which was in concurrence with previous findings. ${ }^{33}$

Mechanistically, EAT volume is closely related to central obesity, which in turn is strongly associated with both insulin resistance and atherosclerotic disease. This process has predominantly been attributed to the enhanced lipolytic activity of visceral fat cells. ${ }^{34}$ Previously, strong associations between EAT volume and both visceral adipose tissue and BMI were demonstrated. The hypothesis that EAT has local pro-atherosclerotic effects is primarily based on its loca- 
tion directly surrounding the coronary arteries, together with its association with CAD. On the other hand, central obesity may lead to insulin resistance and DM2, resulting in systemically accelerated atherosclerotic effects. Some large studies indicate that central body fat outweighs or equals epicardial fat as risk factor for atherosclerosis. ${ }^{4,25}$ In this case-control study, we have investigated the role of EAT as a clinical predictor for CAD in different glycaemic subgroups. Therefore, identifying causal mechanistic relationships was outside the scope of this investigation.

\section{Study limitations}

The current study provides insight in the clinical relevance of EAT volume measurement in stable chest pain patients referred for CCTA. It is a single center study, investigating a limited amount of patients. As we investigated a symptomatic patient group, these results might not be generally applicable. For instance, EAT volume may show higher predictive value in other patient categories or asymptomatic subjects. Therefore, we cannot rule out that EAT volume might improve residual risk stratification in certain populations. The endpoint of CAD was determined by CCTA. Especially for stenosis severity, it is not considered the gold standard and there is still a tendency to overestimation as compared to invasive angiography. As a cross-sectional study, it does not provide insight in the mechanisms behind EAT volume and extent of CAD. We did not measure waist circumference or visceral adipose tissue in this study, although this parameter is important for identification of local versus systemic effects of adipose tissue. Instead we investigated traditional cardiovascular risk factors including BMI, which is a general accepted parameter reflecting systemic body fat, and above all, widely used clinically. The mechanisms of EAT volume potentially resulting in CAD and the role of DM2 in this process should be further investigated in larger clinical trials.

\section{Conclusion}

A clear association was found between EAT volume and both presence and extent of CAD, in non-diabetic patients as well as in patients with IFG and DM2. However, EAT did not independently predict for CAD in our population of stable chest pain patients. Traditional risk factors including DM2 status were the most important independent predictors for CAD. Therefore, the clini- 
cal application of EAT measurement in this patient category would not further benefit risk assessment for $\mathrm{CAD}$ at this moment.

\section{References}

1. Verhagen SN, Visseren FL. Perivascular adipose tissue as a cause of atherosclerosis. Atherosclerosis 2011;214:3-10.

2. Ding J, Kritchevsky SB, Harris TB, Burke GL, Detrano RC, Szklo M, et al. The association of pericardial fat with calcified coronary plaque. Obesity (Silver Spring) 2008;16:1914-9.

3. Greif M, Becker A, von Ziegler F, Lebherz C, Lehrke M, Broedl UC, et al. Pericardial adipose tissue determined by dual source CT is a risk factor for coronary atherosclerosis. Arterioscler Thromb Vasc Biol 2009;29:781-6.

4. Mahabadi AA, Massaro JM, Rosito GA, Levy D, Murabito JM, Wolf PA, et al. Association of pericardial fat, intrathoracic fat, and visceral abdominal fat with cardiovascular disease burden: the Framingham Heart Study. Eur Heart J 2009;30:850-6.

5. Ding J, Hsu FC, Harris TB, Liu Y, Kritchevsky SB, Szklo M, et al. The association of pericardial fat with incident coronary heart disease: the Multi-Ethnic Study of Atherosclerosis (MESA). Am J Clin Nutr 2009;90:499-504.

6. Cheng VY, Dey D, Tamarappoo B, Nakazato R, Gransar H, Miranda-Peats R, et al. Pericardial fat burden on ECG-gated noncontrast CT in asymptomatic patients who subsequently experience adverse cardiovascular events. JACC Cardiovasc Imaging 2010;3:35260.

7. Baker AR, Silva NF, Quinn DW, Harte AL, Pagano D, Bonser RS, et al. Human epicardial adipose tissue expresses a pathogenic profile of adipocytokines in patients with cardiovascular disease. Cardiovasc Diabetol 2006;5:1.

8. Mazurek T, Zhang L, Zalewski A, Mannion JD, Diehl JT, Arafat H, et al. Human epicardial adipose tissue is a source of inflammatory mediators. Circulation 2003;108:2460-6.

9. Mellbin LG, Anselmino M, Ryden L. Diabetes, prediabetes and cardiovascular risk. Eur J Cardiovasc Prev Rehabil 2010;17 Suppl 1:S9-14.

10. Haffner SM, Lehto S, Ronnemaa T, Pyorala K, Laakso M. Mortality from coronary heart disease in subjects with type 2 diabetes and in nondiabetic subjects with and without prior myocardial infarction. N Engl J Med 1998;339:229-34.

11. Hoff JA, Quinn L, Sevrukov A, Lipton RB, Daviglus M, Garside DB, et al. The prevalence of coronary artery calcium among diabetic individuals without known coronary artery disease. J Am Coll Cardiol 2003;41:1008-12. 
12. Goraya TY, Leibson CL, Palumbo PJ, Weston SA, Killian JM, Pfeifer EA, et al. Coronary atherosclerosis in diabetes mellitus: a population-based autopsy study. J Am Coll Cardiol 2002;40:946-53.

13. Iacobellis G, Barbaro G, Gerstein HC. Relationship of epicardial fat thickness and fasting glucose. Int J Cardiol 2008;128:424-6.

14. Wang CP, Hsu HL, Hung WC, Yu TH, Chen YH, Chiu CA, et al. Increased epicardial adipose tissue (EAT) volume in type 2 diabetes mellitus and association with metabolic syndrome and severity of coronary atherosclerosis. Clin Endocrinol (Oxf) 2009;70:876-82.

15. Report of the expert committee on the diagnosis and classification of diabetes mellitus. Diabetes Care 2003;26 Suppl 1:S5-20.

16. Alberti KG, Zimmet PZ. Definition, diagnosis and classification of diabetes mellitus and its complications. Part 1: diagnosis and classification of diabetes mellitus provisional report of a WHO consultation. Diabet Med 1998;15:539-53.

17. Agatston AS, Janowitz WR, Hildner FJ, Zusmer NR, Viamonte M, Jr., Detrano R. Quantification of coronary artery calcium using ultrafast computed tomography. J Am Coll Cardiol 1990;15:827-32.

18. Pontone G, Andreini D, Bartorelli AL, Cortinovis S, Mushtaq S, Bertella E, et al. Diagnostic accuracy of coronary computed tomography angiography: a comparison between prospective and retrospective electrocardiogram triggering. J Am Coll Cardiol 2009;54:346-55.

19. Hausleiter J, Meyer T, Hadamitzky M, Huber E, Zankl M, Martinoff S, et al. Radiation dose estimates from cardiac multislice computed tomography in daily practice: impact of different scanning protocols on effective dose estimates. Circulation 2006;113:1305-10.

20. Yoshizumi T, Nakamura T, Yamane M, Islam AH, Menju M, Yamasaki K, et al. Abdominal fat: standardized technique for measurement at CT. Radiology 1999;211:283-6.

21. Park MJ, Jung JI, Oh YS, Youn HJ. Assessment of Epicardial Fat Volume With ThresholdBased 3-Dimensional Segmentation in CT: Comparison With the 2-Dimensional Short Axis-Based Method. Korean Circ J 2010;40:328-33.

22. Austen WG, Edwards JE, Frye RL, Gensini GG, Gott VL, Griffith LS, et al. A reporting system on patients evaluated for coronary artery disease. Report of the Ad Hoc Committee for Grading of Coronary Artery Disease, Council on Cardiovascular Surgery, American Heart Association. Circulation 1975;51(4 Suppl):5-40.

23. Raff GL, Abidov A, Achenbach S, Berman DS, Boxt LM, Budoff MJ, et al. SCCT guidelines for the interpretation and reporting of coronary computed tomographic angiography. J Cardiovasc Comput Tomogr 2009;3:122-36.

24. Min JK, Shaw LJ, Devereux RB, Okin PM, Weinsaft JW, Russo DJ, et al. Prognostic value of multidetector coronary computed tomographic angiography for prediction of all-cause mortality. J Am Coll Cardiol 2007;50:1161-70. 
25. Rosito GA, Massaro JM, Hoffmann U, Ruberg FL, Mahabadi AA, Vasan RS, et al. Pericardial fat, visceral abdominal fat, cardiovascular disease risk factors, and vascular calcification in a community-based sample: the Framingham Heart Study. Circulation 2008;117:605-13.

26. Gorter PM, de Vos AM, van der Graaf Y, Stella PR, Doevendans PA, Meijs MF, et al. Relation of epicardial and pericoronary fat to coronary atherosclerosis and coronary artery calcium in patients undergoing coronary angiography. Am J Cardiol 2008;102:380-5.

27. Capes SE, Hunt D, Malmberg K, Gerstein HC. Stress hyperglycaemia and increased risk of death after myocardial infarction in patients with and without diabetes: a systematic overview. Lancet 2000;355:773-8.

28. Bettencourt N, Toschke AM, Leite D, Rocha J, Carvalho M, Sampaio F, et al. Epicardial adipose tissue is an independent predictor of coronary atherosclerotic burden. Int J Cardiol 2012;158:26-32.

29. Vliegenthart R, Oudkerk M, Hofman A, Oei HH, van Dijck W, van Rooij FJ, et al. Coronary calcification improves cardiovascular risk prediction in the elderly. Circulation 2005;112:572-7.

30. Motoyama S, Sarai M, Harigaya H, Anno H, Inoue K, Hara T, et al. Computed tomographic angiography characteristics of atherosclerotic plaques subsequently resulting in acute coronary syndrome. J Am Coll Cardiol 2009;54:49-57.

31. Alexopoulos N, McLean DS, Janik M, Arepalli CD, Stillman AE, Raggi P. Epicardial adipose tissue and coronary artery plaque characteristics. Atherosclerosis 2010;210:150-4.

32. Oka T, Yamamoto H, Ohashi N, Kitagawa T, Kunita E, Utsunomiya H, et al. Association between epicardial adipose tissue volume and characteristics of non-calcified plaques assessed by coronary computed tomographic angiography. Int J Cardiol 2012;161:45-9.

33. Pundziute G, Schuijf JD, Jukema JW, van Werkhoven JM, Nucifora G, Decramer I, et al. Type 2 diabetes is associated with more advanced coronary atherosclerosis on multislice computed tomography and virtual histology intravascular ultrasound. J Nucl Cardiol 2009; 16:376-83.

34. Matsuzawa Y. Therapy Insight: adipocytokines in metabolic syndrome and related cardiovascular disease. Nat Clin Pract Cardiovasc Med 2006;3:35-42. 


\section{Chapter}

\section{Coronary Anomalies with and without an Inter-arterial Course as detected by Cardiac CT Angiography: Differences in Coronary Dimensions and Clinical Follow-up?}

Mathijs O Versteylen, Ivo A. Joosen, Leonard Hofstra

Dirk S. Brandsma, Mathijs O. Versteylen, Ivo A. Joosen, Casper Mihl, Marco Das, Joachim E. Wildberger, Harry J. Crijns, Bas L. Kietselaer 


\begin{abstract}
Objectives To study the dimensions and short-term follow-up of coronary anomalies with and without an inter-arterial course.

Methods We studied all patients referred for cardiac computed tomographic angiography (CCTA) from April 2007 to September 2011. Coronary arteries originating from an alternate sinus of Valsalva were defined as anomalous, and coronary dimensions were measured. Patients were followed-up for cardiac events for a mean of $38 \pm 13$ months.
\end{abstract}

Results CCTA was performed in 2,914 patients. Of these patients, $26(0.9 \%)$ had a coronary artery with an anomalous origin, 15 had an inter-arterial course. The angle of origin of inter-arterial coronaries was less sharp as compared with non inter-arterial coronaries (82 \pm 11 degrees vs. $67 \pm 15$ degrees, $\mathrm{p}=0.005)$. The shape of inter-arterial coronaries was more often ellipsoid $(73 \%$ vs. $9 \%, \mathrm{p}=0.002)$, ostial area was smaller $\left(7.1 \pm 1.7 \mathrm{~mm}^{2}\right.$ vs. $10.6 \pm 4.3 \mathrm{~mm}^{2}$, $\mathrm{p}=0.009)$. However, inter-arterial anomalous coronaries had larger distal areas $\left(12.9 \pm 6.2 \mathrm{~mm}^{2}\right.$ vs. $\left.8.0 \pm 1.8 \mathrm{~mm}^{2}, \mathrm{p}=0.02\right)$ and consequently a smaller area-index $(0.6 \pm 0.2$ vs. $1.3 \pm 0.4, \mathrm{p}<0.001)$. During follow-up, 5 events occurred ( 3 in the inter-arterial group), which could not be contributed to the anomalous course.

Conclusion We did not objectify differences between anomalies with and without an inter-arterial course during follow-up. However, inter-arterial anomalies show less favourable coronary dimensions. 


\section{INTRODUCTION}

Case report: monocoronary system with malignant course of the LAD.

A 43-year old male is referred to the cardiologist with near-syncopal episodes during exercise. The patient has an intermediate cardiovascular risk profile and an exercise-ECG is shows no signs of ischemia. To further rule out coronary artery disease, cardiac CT is performed. CT-angiography reveals a monocoronary system, with the LAD and LCX originating from the right coronary artery (Figure 1, panel A). A superior view on volume-rendering imaging shows the LAD following a so-called malignant tract, flanked by the ascending aorta and the pulmonary artery (panel B). Neither stenoses nor plaques are identified. This rare coronary anomaly is associated with ischemia and sudden death, hypothetically caused by external compression of the LAD by the aorta and pulmonary artery during exercise, predominantly in young patients and often precipitated by exercise. As this patient shows an excellent exercise tolerability without any signs of ischemia and his age is well above 30 years, he is advised to refrain from exercise and followed up by his cardiologist.

Figure 1. Monocoronary artery originating from the right sinus of Valsalva.
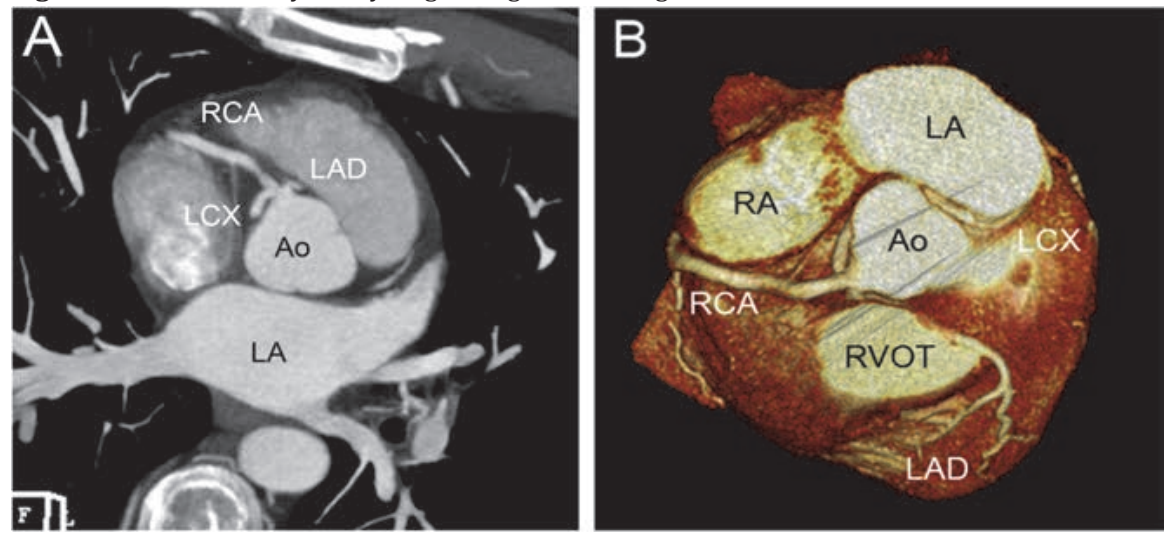

Panel A; trifurcation of the monocoronary artery. Panel B; 3-dimensional reconstruction showing superior view of the coronary anatomy.

\section{General introduction}

Coronary anomalies can be accurately detected by cardiac computed tomographic angiography (CCTA). ${ }^{1-3}$ Due to widespread implementation of CCTA, an increasing number of coronary anomalies may be detected. Currently, con- 
sensus about clinical consequences of such findings is lacking, and pathophysiologic mechanisms not fully understood.

Coronary anomalies have been proposed as a common cause of sudden death, especially in the young ${ }^{4}$ and athletes. ${ }^{5}$ Primarily, the presence of an interarterial course has been associated with the occurrence of sudden cardiac death. ${ }^{4}$ In particular when the left coronary artery (LCA) originates from the right sinus of Valsalva (RSV) or right coronary artery (RCA).,7 Other characteristics associated with adverse outcomes are coronary dimensions, such as an ellipsoid shape or acute angle of origin. ${ }^{8}$ The relation between these coronary dimensions and the presence of an inter-arterial course is unclear.

We therefore investigated the prevalence of coronary anomalies originating from an alternate sinus of Valsalva in a population undergoing CCTA. The main goal of our study was to investigate potential differences in coronary dimensions between patients with- and without an inter-arterial course. Also, we investigated the value of these coronary anomaly characteristics to predict for short-term cardiac events.

\section{METHODS}

\section{Study population}

We analyzed 2,914 consecutive patients referred from the cardiology outpatient department for CCTA because of stable chest discomfort symptoms, suspected for coronary artery disease (CAD). We excluded patients with a non-diagnostic CCTA. Written informed consent was waived because data were analyzed anonymously in accordance with IRB guidelines. This study complies with the ethical principles of the Declaration of Helsinki.

\section{CCTA acquisition and assessment}

Scans were performed using either a 64-slice multidetector CT-scanner (Brilliance 64, Philips Healthcare, Best, the Netherlands) or a second generation dual-source CT-scanner (Somatom Definition Flash, Siemens Medical Solutions, Forchheim, Germany). The 64-slice scanner used a 64 x $0.625 \mathrm{~mm}$ slice collimation, gantry rotation time of $420 \mathrm{~ms}$, and a tube voltage of 80 or $120 \mathrm{kV}$, depending on patient weight. The second generation dual-source scanner used a slice collimation of $2 \times 128 \times 0.6 \mathrm{~mm}$, gantry rotation time of $280 \mathrm{~ms}$ and tube voltage of 80, 100 or $120 \mathrm{kV}$, depending on patient weight. Patients received 50 
mg Metoprolol tartrate (AstraZeneca, Zoetermeer, The Netherlands) orally, two hours before CCTA. When indicated, an additional dose of 5-20 mg Metoprolol was administered intravenously on site, in an attempt to lower the heart rate $<60$ beats per minute (bpm). All patients received $0.8 \mathrm{mg}$ Nitroglycerin spray (Pohl-Boskamp, Hohenlockstedt, Germany) sublingually just prior to CCTA. The CCTA protocol was more extensively described before. ${ }^{9}$

A native scan using $120 \mathrm{kV}$ was performed to determine the calcium score according to the Agatston method. ${ }^{10}$ Using the American Heart Association 16 coronary segments model, CT angiograms were independently analyzed by a cardiologist and radiologist who were both experienced in reading CCTA and blinded to clinical information. In case of disagreement, consensus was reached by reviewing the images jointly. The coronary artery tree was assessed using the source images on the provided software (Cardiac Comprehensive Analysis, Philips). The presence of significant CAD was defined as having one or more coronary plaques with $\geq 50 \%$ luminal stenosis. Mean estimated radiation dose was calculated by multiplying the dose length product by the conversion factor of $0.014 \mathrm{mSv} /(\mathrm{mGy} \mathrm{xcm})$ for the thorax. ${ }^{11,12}$

\section{Anomaly assessment}

CT scans were reviewed for coronary anomalies, defined as a coronary origin from an alternate sinus of Valsalva. The shape of the anomalous origin was visually assessed to be circular or ellipsoid. In addition, the course of the anomalous coronary was described, discriminating between inter-arterial and non inter-arterial courses. Using the area measurement tool (Cardiac Comprehensive Analysis, Philips), the area of the proximal coronary lumen was measured directly after the origin and distally at the point with the largest diameter. An area-index was calculated by dividing the proximal area by the distal area, to assess luminal narrowing. We also measured the angle of origin between the anomalous origin and aorta or other coronary artery, depending whether the anomaly originated from the aorta or from another coronary artery, respectively. Invasive coronary angiography (ICA) including fractional flow reserve (FFR) measurement was performed at the discretion of the treating cardiologist. FFR was measured using a coronary guidewire (PressureWire Certus or PressureWire Aeris, St. Jude Medical). The hemodynamic severity of the stenosis was assessed after intracoronary administration of $120 \mathrm{mcg}$ adenosine, to induce hyperemia. FFR measurements below 0.80 were considered to be hemodynamically significant. 


\section{Follow-up}

Electronic patient records were monitored for all-cause mortality, myocardial infarction and revascularization procedures. Myocardial infarction was defined as a rise and/or fall in troponin $\mathrm{T}$, with at least one value above the 99th percentile $(0.01 \mu \mathrm{g} / \mathrm{L})$, combined with either typical chest pain, ECG changes suggestive for ischemia, development of pathological Q-waves, or imaging evidence of loss of myocardium or regional wall motion abnormality. ${ }^{13}$ Revascularization was defined as a percutaneous coronary intervention (PCI) or coronary artery bypass grafting $(\mathrm{CABG})$ procedure.

\section{Statistics}

Data were analyzed using SPSS 19.0 (SPSS Inc., Chicago, IL, USA). Continuous variables were reported as means and standard deviations (SD), or median and interquartile range (IQR) for nonparametric parameters. Proportions (\%) were used for categorical values. Fisher's exact test or chi-square test was used to measure differences between categorical values, depending on expected count. For continuous variables, independent $\mathrm{t}$-test was performed to assess difference between mean values. All p-values were 2 -sided, and a value below 0.05 was considered significant.

\section{RESULTS}

\section{Study population}

A total of 2,914 patients underwent CCTA. Of these patients, $26(0.9 \%)$ had a coronary artery with an anomalous origin, which were subject to this study. This group of patients had a mean age of $56 \pm 14$ years, $19(73 \%)$ were male. Baseline characteristics as well as indications for CCTA are summarized in Table 1 
Table 1. Baseline characteristics

\begin{tabular}{lcccc} 
& $\begin{array}{c}\text { Total } \\
(\mathrm{n}=26)\end{array}$ & $\begin{array}{c}\text { Inter-arterial } \\
(\mathrm{n}=15)\end{array}$ & $\begin{array}{c}\text { Non inter- } \\
\text { arterial } \\
(\mathrm{n}=11)\end{array}$ & P-value \\
\hline Age (years) & $56 \pm 14$ & $58 \pm 14$ & $53 \pm 14$ & 0.35 \\
Male gender & $19(73 \%)$ & $10(67 \%)$ & $9(82 \%)$ & 0.66 \\
BMI (kg/m2) & $27 \pm 5$ & $27 \pm 5$ & $28 \pm 6$ & 0.43 \\
Positive family history & $6(23 \%)$ & $3(20 \%)$ & $3(27 \%)$ & 1.00 \\
Diabetes Mellitus & $6(23 \%)$ & $4(27 \%)$ & $2(18 \%)$ & 1.00 \\
Smoking & $4(15 \%)$ & $3(20 \%)$ & $1(9 \%)$ & 0.61 \\
Systolic blood pressure (mmHg) & $145 \pm 22$ & $142 \pm 20$ & $151 \pm 24$ & 0.28 \\
Calcium score & $9(0-94)$ & $4(0-144)$ & $20(1-50)$ & 0.65 \\
$\geq 50 \%$ stenosis & $5(19 \%)$ & $4(27 \%)$ & $1(9 \%)$ & 0.36 \\
Radiation dose (mSv) & $5.8 \pm 4.9$ & $7.2 \pm 5.7$ & $3.5 \pm 1.0$ & 0.11 \\
Chest pain & $17(65 \%)$ & $11(58 \%)$ & $6(55 \%)$ & 0.42 \\
\hline
\end{tabular}

Values are presented as numbers (\%), mean \pm standard deviation except calcium score which is presented as median (IQR). BMI=body mass index,

\section{Coronary anomalies assessment and measurements}

Of 26 anomalies, 15 had an inter-arterial course. A typical example is shown in Figure 2.

Figure 2. Example of anomalous RCA originating from the LSV, with inter-arterial course.
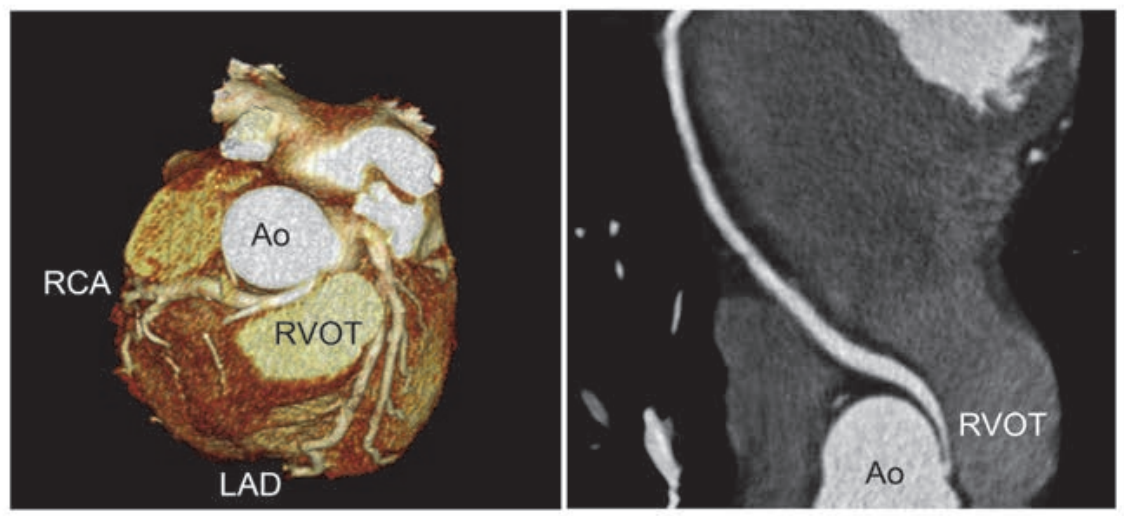

The left panel shows a volume rendered reconstruction showing the course of the anomaly. The right panel shows a multiplanar reconstruction, clearly showing a small ostium and coronary caliber throughout the inter-arterial course. Ao=aorta ascendens, LAD=left anterior descending artery, RCA=right coronary artery, RVOT= right ventricular outflow tract. 
In 13 of these patients the right coronary artery (RCA) originated from the left sinus of Valsalva (LSV), in 1 patient the RCA originated from the left anterior descending artery (LAD) and in 1 patient the LAD originated from the RCA (in this variant the left circumflex artery $[\mathrm{LCx}]$ also originated from the RCA, but did not have an inter-arterial course). Additionally, in 11 patients the aberrant coronary artery did not have an inter-arterial course. The LCx originated from the RSV in 6 cases, from the RCA in 3 cases, in 1 case the LAD and LCx both originated from the RSV and in 1 case both the LCx and the LAD originated from the RCA. All different coronary anomalies are listed and further described in Table 2.

Anomalies with an inter-arterial course more often showed an ellipsoid origin and a larger angle of origin from the sinus of Valsalva as compared with anomalies without inter-arterial course. In addition, anomalies with an interarterial course had a significantly smaller proximal lumen area and a larger distal lumen area, resulting in a smaller lumen area-index (Table 3). In only 3 patients with an inter-arterial course of the anomalous coronary artery, FFR measurement was performed. In 1 patient, FFR indicated a significant stenosis whereupon the anomalous coronary artery was surgically re-implanted.

\section{Follow-up}

The patient group was followed-up for a mean of $38 \pm 13$ months (range $11-$ 51 months). During this follow-up period, 5 (19\%) events occurred. Two patients died, 1 due to end-stage heart failure and 1 as a result of suicide. In 1 patient, PCI of an atherosclerotic segment in the aberrant LCx was performed (segment in previous CCTA was regarded as normal). In 2 patients, a myocardial infarction was reported. Unfortunately, in one of these patients the infarction was a complication of FFR measurement, during which a dissection of the RCA occurred. In the other patient myocardial infarction was based on occlusion of the non-aberrant coronary artery. 
Table 2. Distribution of coronary anomalies

\begin{tabular}{|c|c|c|c|c|c|c|c|}
\hline $\begin{array}{l}\text { Patient } \\
\text { number }\end{array}$ & Age/ sex & $\begin{array}{l}\text { Coronary } \\
\text { anomaly }\end{array}$ & $\begin{array}{l}\text { Inter- } \\
\text { arterial }\end{array}$ & CAD & FFR & Surgery & Event \\
\hline 1 & $54 / \mathrm{M}$ & LCx from RSV & no & no & - & no & - \\
\hline 2 & $41 / \mathrm{M}$ & RCA from LSV & yes & yes & - & no & - \\
\hline 3 & $57 / \mathrm{M}$ & LCx from RCA & no & no & - & no & - \\
\hline 4 & $77 / \mathrm{M}$ & RCA from LSV & yes & yes & - & no & NSTEMI \\
\hline 5 & $62 / M$ & RCA from LSV & yes & no & positive & yes & - \\
\hline 6 & $42 / \mathrm{M}$ & LCx from RCA & no & no & - & no & - \\
\hline 7 & $18 / \mathrm{M}$ & LCx from RSV & no & no & - & no & - \\
\hline 8 & $50 / \mathrm{M}$ & LCx from RSV & no & no & - & no & - \\
\hline 9 & $70 / \mathrm{M}$ & LCA from RSV & no & no & - & no & - \\
\hline 10 & $64 / \mathrm{M}$ & LCx from RSV & no & yes & - & no & - \\
\hline 11 & $63 / \mathrm{W}$ & LCx from RSV & no & no & - & no & PCI LCx \\
\hline 12 & $59 / \mathrm{M}$ & LCx from RSV & no & no & - & no & Death \\
\hline 13 & $49 / \mathrm{W}$ & LCx from RCA & no & no & - & no & - \\
\hline 14 & $50 / \mathrm{M}$ & RCA from LSV & yes & no & - & no & - \\
\hline 15 & $64 / \mathrm{M}$ & RCA from LSV & yes & no & - & no & - \\
\hline 16 & $69 / M$ & RCA from LSV & yes & yes & negative & no & STEMI \\
\hline 17 & $40 / W$ & RCA from LSV & yes & no & - & no & - \\
\hline 18 & $52 / \mathrm{M}$ & RCA from LSV & yes & yes & - & no & - \\
\hline 19 & $52 / \mathrm{W}$ & RCA from LAD & yes & no & - & no & - \\
\hline 20 & $43 / \mathrm{M}$ & $\begin{array}{l}\text { LAD from RCA } \\
\text { (LCx from RCA) }\end{array}$ & yes & no & negative & no & - \\
\hline 21 & $86 / \mathrm{W}$ & RCA from LSV & yes & no & - & no & Death \\
\hline 22 & $54 / \mathrm{W}$ & $\begin{array}{l}\text { LAD from RCA } \\
\text { (LCx from RCA) }\end{array}$ & no & no & - & no & - \\
\hline 23 & $67 / \mathrm{W}$ & RCA from LSV & yes & no & - & no & - \\
\hline 24 & $59 / \mathrm{M}$ & RCA from LSV & yes & no & - & no & - \\
\hline 25 & $44 / \mathrm{W}$ & RCA from LSV & yes & no & - & no & - \\
\hline 26 & $63 / \mathrm{M}$ & RCA from LSV & yes & no & - & no & - \\
\hline
\end{tabular}

$\mathrm{CAD}=$ coronary artery disease, $\mathrm{FFR}=$ fractional flow reserve, $\mathrm{LAD}=$ left anterior descending, $\mathrm{LCx}=\mathrm{left}$ circumflex, LSV=left sinus of Valsalva, RCA=right coronary artery, RSV=right sinus of Valsalva, NSTEMI=non ST-elevation myocardial infarction. 
Table 3. Dimension assessment of coronary anomalies.

\begin{tabular}{lccc} 
& $\begin{array}{c}\text { Inter-arterial } \\
(\mathrm{n}=15)\end{array}$ & $\begin{array}{c}\text { Non interarterial } \\
(\mathrm{n}=11)\end{array}$ & P-value \\
\hline Ellipsoid origin & $11(73 \%)$ & $1(9 \%)$ & 0.002 \\
Angle of origin $\left({ }^{\circ}\right)$ & $82 \pm 11$ & $67 \pm 15$ & 0.005 \\
Area ostium $\left(\mathrm{mm}^{2}\right)$ & $7.1 \pm 1.7$ & $10.6 \pm 4.3$ & 0.009 \\
Area distal coronary $(\mathrm{mm} 2)$ & $12.9 \pm 6.2$ & $8.0 \pm 1.8$ & 0.02 \\
Area-index & $0.63 \pm 0.24$ & $1.32 \pm 0.44$ & $<0.001$ \\
\hline
\end{tabular}

Values are presented as numbers (\%), mean \pm standard deviation.

\section{DISCUSSION}

In this study, we present new data showing that anomalies with an inter-arterial course had significantly smaller coronary dimensions as compared with non inter-arterial course anomalies. The clinical presentation between patients with and without inter-arterial anomalies did not differ, nor did follow-up. In $5 \mathrm{pa}-$ tients an event occurred during follow up. However, none of these events have likely been caused by the coronary anomaly. Although research on the prognosis of adult patients with a coronary anomaly is limited, these results are in line with previous studies. ${ }^{11,13}$

Because our study design included patients that were suspected of having a coronary anomaly, we cannot reliably estimate the prevalence in the nonselected population. However, prevalence of coronary anomalies in patients undergoing CCTA to rule out CAD must be low. We report a rate of about $1 \%$ for coronary arteries originating from an alternate sinus of Valsalva. As can be expected, this is slightly higher than in most previous studies (Table 4).3,14-17 Despite our inclusion pattern, we hereby present a series of patients in which coronary anomalies are extensively described and follow-up data was collected.

The mechanism of sudden cardiac death in coronary anomalies has not been fully elucidated. An inter-arterial course might play a role and has been associated with cardiovascular events. ${ }^{4}$ In our study, the origin of inter-arterial anomalies was more often of ellipsoid shape and had a smaller cross-sectional area as compared with anomalies without inter-arterial course. However, due to the small numbers and overall low event rate, the implications of these findings remain uncertain. Also, inter-arterial arteries had a small mean area-index, implicating that the proximal lumen of inter-arterial anomalies are relatively small. This could possibly compromise blood flow and result in exercise-induced hypoxia. The smallest area-index measured was 0.26 , in the only patient who underwent coronary re-implantation. The hemodynamic significance of this 
stenosis was confirmed by FFR measurement. We could therefore hypothesize that area-index might be a potential marker for hemodynamic severity of the inter-arterial course.

A sharp angle of origin has also been suggested to play a role in the risk of events. ${ }^{8}$ We, however, found significantly larger angles of origin in coronary anomalies with an inter-arterial course. This finding seems not in accordance with risk previously contributed to angle of origin.

Currently, the ACC/AHA 2008 guidelines for the management of adults with congenital heart disease advise for patients with coronary anomalies to evaluate patency, function and anatomic integrity at least once in adulthood. CT or MRI is suggested to be used for initial screening. Re-implantation surgery is advised in case of an inter-arterial left main, surgical intervention for interarterial LAD is optional. ${ }^{18}$ We described one patient with an inter-arterial LAD. However, since no hemodynamic significance could be objectified during FFR, a conservative treatment strategy was followed and no events were reported.

The very low event rate reported in the present study might seem counterintuitive. In contrast to other studies, we did not objectify an association between inter-arterial course and subsequent events. Previous studies have shown an association between coronary anomalies and sudden cardiac death especially in younger patients ( $<30$ years) and young athletes. $4,5,7,19,20$ The chance of sudden death due to coronary anomalies after the age of 30 decreases significantly. ${ }^{19}$ Therefore, our population might suffer from selection bias, because younger patients and athletes are infrequently referred for CCTA. In other words, it is possible that we investigated the surviving, older population. In addition, it has been reported that only $12 \%$ to $33 \%$ of sudden cardiac death in the young are caused by coronary anomalies. ${ }^{21}$ Our data suggest that coronary anomalies in a population that is referred for CCTA have a relatively benign short-term prognosis.

Although coronary anomalies have been associated with sudden cardiac death in the young, not much is known about prognosis of coronary anomalies in an older population. With the increasing age of population in Western societies, the amount of anomalies detected will likely increase. Hence more insight in the clinical consequences and pathophysiology is desirable. Hereby we present new information, linking coronary anomaly course and dimensions in the adult population. Importantly, in this population the short-term prognosis was good. 


\section{Limitations}

This is a single-centre, retrospective analysis of all consecutive patient who underwent CCTA. Due to the nature of the study, the results may suffer from referral bias. Some patients were referred after invasive conventional angiography. However, since we included all consecutive patients, these data do represent daily clinical practice. We described 26 patents with a coronary anomaly, which represents a relatively large group. However, only a few events occurred during follow-up. Therefore we suggest that multicentre studies with longer follow-up times and coronary measurements could help to elucidate the prognosis and pathophysiology of coronary anomalies in a larger patient group.

\section{Conclusion}

The prevalence of anomalous coronary arteries in this middle-aged population referred for CCTA was low. We did not objectify differences between anomalies with and without an inter-arterial course in clinical presentation or in cardiac event rate during follow-up. However, inter-arterial coronaries showed a significant smaller area of ostium and area-index while having a significantly larger angle of origin as compared with anomalies without an inter-arterial course.

\section{References}

1. Cademartiri F, La Grutta L, Malago R, et al. (2008) Prevalence of anatomical variants and coronary anomalies in 543 consecutive patients studied with 64-slice CT coronary angiography. Eur Radiol 18:781-91.

2. Berbarie RF, Dockery WD, Johnson KB, Rosenthal RL, Stoler RC, Schussler JM. (2006) Use of multislice computed tomographic coronary angiography for the diagnosis of anomalous coronary arteries. Am J Cardiol 98:402-6.

3. Srinivasan KG, Gaikwad A, Kannan BR, Ritesh K, Ushanandini KP (2008). Congenital coronary artery anomalies: diagnosis with 64 slice multidetector row computed tomography coronary angiography: a single-centre study. J Med Imaging Radiat Oncol.:148-54.

4. Taylor AJ, Rogan KM, Virmani R. (1992) Sudden cardiac death associated with isolated congenital coronary artery anomalies. J Am Coll Cardiol 20:640-7. 
5. Maron BJ, Thompson PD, Puffer JC, et al. (1996) Cardiovascular preparticipation screening of competitive athletes. A statement for health professionals from the Sudden Death Committee (clinical cardiology) and Congenital Cardiac Defects Committee (cardiovascular disease in the young), American Heart Association. Circulation 94:850-6.

6. Eckart RE, Scoville SL, Campbell CL, et al. (2004) Sudden death in young adults: a 25-year review of autopsies in military recruits. Ann Intern Med 141:829-34.

7. Basso C, Maron BJ, Corrado D, Thiene G. (2000) Clinical profile of congenital coronary artery anomalies with origin from the wrong aortic sinus leading to sudden death in young competitive athletes. J Am Coll Cardiol 35:1493-501.

8. Virmani R, Chun PK, Goldstein RE, Robinowitz M, McAllister HA. (1984) Acute takeoffs of the coronary arteries along the aortic wall and congenital coronary ostial valve-like ridges: association with sudden death. J Am Coll Cardiol 3:766-71.

9. Versteylen MO, Joosen IA, Shaw LJ, Narula J, Hofstra L. (2011) Comparison of Framingham, PROCAM, SCORE, and Diamond Forrester to predict coronary atherosclerosis and cardiovascular events. J Nucl Cardiol 18:904-11.

10. Agatston AS, Janowitz WR, Hildner FJ, Zusmer NR, Viamonte M, Jr., Detrano R. (1990) Quantification of coronary artery calcium using ultrafast computed tomography. J Am Coll Cardiol 15:827-32.

11. Jessen KA, Shrimpton PC, Geleijns J, Panzer W, Tosi G. (1999)Dosimetry for optimisation of patient protection in computed tomography. Applied Radiation and Isotopes 50:165172.

12. Shrimpton PC. (2004) Assessment of patient dose in CT. National Radiological Protection Board, Oxon. Available via http://www.msct.eu/PDF_FILES/Appendix\%20paediatric\%20CT\%20Dosimetry.pdf Accessed 19 June 2013

13. Thygesen K, Alpert JS, White HD. (2007) Universal definition of myocardial infarction. J Am Coll Cardiol 50:2173-95.

14. Bazzocchi G, Romagnoli A, Sperandio M, Simonetti G. (2011) Evaluation with 64-slice CT of the prevalence of coronary artery variants and congenital anomalies: a retrospective study of 3,236 patients. Radiol Med116:675-89.

15. Girzadas M, Varga P, Dajani K. (2009) A single-center experience of detecting coronary anomalies on 64-slice computed tomography. Journal of cardiovascular medicine 10:842-7.

16. Tariq R, Kureshi SB, Siddiqui UT, Ahmed R. (2012) Congenital anomalies of coronary arteries: Diagnosis with 64 slice multidetector CT. Eur J Radiol 81:1790-7.

17. Zhang LJ, Yang GF, Huang W, Zhou CS, Chen P, Lu GM.(2010) Incidence of anomalous origin of coronary artery in 1879 Chinese adults on dual-source CT angiography. Neth Heart J 18:466-70. 
18. Warnes CA, Williams RG, Bashore TM, et al. (2008) ACC/AHA 2008 Guidelines for the Management of Adults with Congenital Heart Disease: Executive Summary: a report of the American College of Cardiology/American Heart Association Task Force on Practice Guidelines (writing committee to develop guidelines for the management of adults with congenital heart disease). Circulation 118:2395-451.

19. Taylor AJ, Byers JP, Cheitlin MD, Virmani R. (1997) Anomalous right or left coronary artery from the contralateral coronary sinus: "high-risk" abnormalities in the initial coronary artery course and heterogeneous clinical outcomes. Am Heart J 133:428-35.

20. Frescura C, Basso C, Thiene G, et al. (1998) Anomalous origin of coronary arteries and risk of sudden death: a study based on an autopsy population of congenital heart disease. Hum Pathol 29:689-95.

21. Chandra N, Bastiaenen R, Papadakis M, Sharma S. (2013) Sudden Cardiac Death in Young Athletes: Practical Challenges and Diagnostic Dilemmas. J Am Coll Cardiol 61:1027-1040. 


\title{
Combined Use of Exercise Electrocardiography, Coronary Calcium Score and Cardiac CT Angiography for the Prediction of Major Cardiovascular Events in Patients Presenting with Stable Chest Pain
}

\author{
Mathijs O. Versteylen, Ivo A. Joosen, Mark H. Winkens, \\ Eduard M. Laufer, Roel J. Snijder, Joachim E. Wildberger, \\ Harry J. Crijns, Jagat Narula, Leonard Hofstra
}

Int J Cardiol. 2013; 167: 121-125. 


\begin{abstract}
Background The usual diagnostic work-up of chest pain patients includes clinical risk profiling and exercise-ECG, possibly followed by additional tests. Recently cardiac computed tomographic angiography (CCTA) has been employed. We evaluated the prognostic value of the combined use of exercise-ECG and CCTA for the development of cardiovascular endpoints.

Methods In 283 patients (143 male, mean age $54 \pm 10$ years) with intermediate pre-test probability for coronary artery disease presenting with stable chest pain, exercise-ECG, CCTA and calcium score were performed. Patients were followed-up for combined endpoint of acute coronary syndrome (ACS) and revascularization.
\end{abstract}

Results After a median follow-up of 769 days (interquartile range 644 - 1007), 6 ACS and 9 revascularizations were recorded. A positive exercise-ECG predicted for the combined endpoint, [hazard ratio (HR) 5.14 (95\% confidence interval (CI) 1.64-16.13), $\mathrm{p}=0.005$ ], as well as a positive calcium score [HR 4.59 (95\% CI 1.30-16.28), $\mathrm{p}=0.02$ ] and a $\geq 50 \%$ stenosis on CCTA [HR 45.82 (95\% CI 6.02-348.54), $\mathrm{p}<0.001$ ]. ROC-analysis showed an area under the curve (AUC) of 0.79 (95\% CI 0.67-0.90) for exercise-ECG, which increased significantly when CCTA was added: 0.91 (95\% CI; 0.86-0.97; $\mathrm{p}=0.006$ ). Multivariable Cox regression showed exercise-ECG predicted independently [HR 3.6, (95\% CI 1.1-11.2), $\mathrm{p}=0.03$ ], as well as CCTA [HR 31.4 (95\% CI 4.0-246.6), p=0.001], but not calcium score [HR 0.6 (95\% CI 0.2-2.3), $\mathrm{p}=0.5$ ].

Conclusions The combined subsequent use of exercise-ECG for functional information and CCTA for anatomical information provides a high diagnostic yield in stable chest pain patients with an intermediate pre-test probability for coronary artery disease. 


\section{INTRODUCTION}

The clinical work-up of patients presenting with stable chest pain symptoms remains a challenge, especially in patients with an intermediate pre-test probability of coronary artery disease (CAD). Usually, traditional risk factors provide initial risk assessment, and in addition exercise-ECG has been widely used in evaluating patients presenting with chest pain despite only fair diagnostic accuracy. The proportion of inconclusive tests is relatively high and the sensitivity, negative predictive value and prognostic abilities low. ${ }^{1}$ Depending on the initial clinical assessment and exercise-ECG results, a combination of stress testing with echocardiography or nuclear perfusion imaging is traditionally recommended. ${ }^{2,3,4}$ Although these imaging tests provide excellent information about the functional significance of the coronary lesions, they are neither ideal for the geographical location of the lesion nor for the assessment of plaque characteristics. Recently, cardiac CT-angiography (CCTA) has gained a prominence for the assessment of patients with suspected CAD and has offered valuable information about the severity of stenotic lesions and plaque characteristics. 5,6 However, even though some studies have suggested the simultaneous registration of contrast passage through myocardium as an indicator of perfusion, ${ }^{7}$ the functional relevance of the anatomical lesions remains unsubstantiated. ${ }^{8}$ The aim of this study was to investigate exercise-ECG as a low-cost supplementation to CCTA for a combined possible yield of anatomic and functional significance of coronary artery lesions.

\section{METHODS}

\section{Study population}

We retrospectively analyzed stable chest pain patients who were referred for exercise-ECG and subsequent CCTA by their cardiologist. In all patients referred for CCTA, pre-test probability for CAD was calculated using DiamondForrester classification. ${ }^{9}$ Patients with a high pre-test probability were not referred for CCTA, consequently our population consisted of patients with a low to intermediate pre-test probability for CAD, which is according to the 2010 appropriateness criteria on CCTA. ${ }^{10}$ Excluded were patients with unstable angina, hemodynamic instability, previous CAD, pregnancy, renal insufficiency, iodine allergy, inconclusive exercise-ECG and/or non-diagnostic CCTA. From July 2007 until November 2009, 407 patients were included, 14(3) had a history 
of CAD, 91(22) had an inconclusive exercise-ECG and 36(9) an inconclusive CCTA (17 patients had both an inconclusive exercise-ECG as an inconclusive CCTA). Eventually 283 subjects were studied. The study protocol conforms to the ethical guidelines of the 1975 Declaration of Helsinki. The Institutional Review Board and ethics committee approved the study and all patients signed informed consent. The authors of this manuscript have certified that they comply with the Principles of Ethical Publishing in the International Journal of Cardiology. ${ }^{11}$

\section{Risk assessment and diagnostics}

In all patients historic details were recorded. Framingham risk score (FRS) was calculated to estimate the 10-year risk of cardiovascular disease, and patients were classified as low risk, intermediate risk or high risk, when FRS was $<5 \%$, between 5 and $20 \%$ or $>20 \%$, respectively. ${ }^{12}$

Exercise-ECG tests were performed per standardized Bruce-protocol, with continuous blood pressure, heart rate and ECG monitoring up to at least 5 minutes into recovery. Tests were classified as inconclusive in patients who did not reach their predicted exercise tolerance $(<85 \%$ of the age- and sexpredicted heart rate or a heart rate pressure product $<18,000)$. A test was considered positive in case of $\geq 1.0 \mathrm{~mm}$ horizontal or downsloping ST-deviation at $80 \mathrm{~ms}$ after the J-point compared to baseline ECG ( $\geq 1.5 \mathrm{~mm}$ was used for upsloping ST-deviation) and/or typical angina and/or ventricular arrhythmia and/or a decrease in systolic blood pressure $>10 \mathrm{mmHg}$. Conclusive tests that did not met any of these criteria were regarded as negative. ${ }^{13}$

CCTA was performed using a 64-slice multi detector computed tomography scanner (Brilliance 64; Philips Healthcare) with a 64 x $0.625 \mathrm{~mm}$ slice collimation, a gantry rotation time of $420 \mathrm{~ms}$, a tube voltage of $80-120 \mathrm{kV}$, depending on the patient's height and weight. In patients with heart rates $<65$ bpm, a prospective-gated "Step and shoot" protocol was used, and in those with heart rates $>65 \mathrm{bpm}$, a retrospective-gated "Helical" protocol with dose modulation was used to obtain the best image quality at minimal radiation dose. ${ }^{14,15}$ Tube current varied from 150-210 mAs and from 600-1000 mAs, for "Step and shoot" and "Helical" protocol, respectively, again depending on patients weight and height. Patients received 5-20 mg of Metoprolol intravenously to lower the heart rate $<65 \mathrm{bpm}$ as well as $0.8 \mathrm{mg}$ sublingual nitroglycerin spray. A native scan was performed to determine the calcium score using the Agatston method. ${ }^{16}$ This was followed by CCTA using contrast agent as de- 
scribed before. ${ }^{17}$ Using the American Heart Association (AHA) in 16 coronary segments model, CT angiograms were independently analyzed by two experienced cardiologists who were blinded to clinical information. ${ }^{18}$ In case of disagreement, consensus was reached by discussion. The coronary artery tree was assessed using the source images on the provided software (Philips Healthcare), and the degree of stenosis was visually defined as insignificant (no lesions, or one or more lesions with diameter stenosis of $<50 \%$ ), or significant (one or more lesions with diameter stenosis $\geq 50 \%$ ). ${ }^{19}$ In addition, a segment score was calculated as follows: mild diameter stenosis $(<50 \%$, score 1$)$, moderate (50$70 \%$, score 2$)$ and severe diameter stenosis ( $>70 \%$, score 3$)$; the total score was calculated as the sum of all score points divided by the number of assessable segments, resulting in a score ranging 0-3. An involvement score was calculated by counting all diseased vessel segments (irrespective of degree of stenosis), and the total score ranged from $0-16 .{ }^{20}$ Plaques were categorized as calcified (exclusively content with density $>130$ Hounsfield units), non-calcified (exclusively content with density $<130$ Hounsfield units), or mixed (characteristics of both calcified and non-calcified plaques).

\section{Follow-up}

Electronic patient records were monitored for cardiovascular death and acute coronary syndrome (ACS), including myocardial infarction and unstable angina. Additionally, the national mortality records were checked. ACS was defined as typical angina pectoris and troponin T elevation $(>0.01 \mu \mathrm{g} / \mathrm{L})$ and ST-segment elevation/depression of $\geq 1 \mathrm{~mm}$, or at least two of these symptoms together with invasive angiographic conformation of a culprit lesion. ${ }^{21}$ Secondary endpoints included elective percutaneous coronary intervention (PCI) and coronary artery bypass graft $(\mathrm{CABG})$ surgery. Revascularization procedures $<60$ days after CCTA were excluded as event, as these would be predominantly CCTA driven. Further, we censored follow-up after the first endpoint, so that the recorded ACS was not a complication of revascularization therapy. Patients were seen by their cardiologist on a regular basis, and all visits were recorded in the electronic patient records.

\section{Statistics}

Data were analyzed using SPSS 17.0. Continuous variables were reported as means and SDs and proportions (\%) were used for categorical values. Time to 
events was presented as mean and interquartile range. All relevant clinical parameters including exercise-ECG, CCTA and calcium score were evaluated for their individual prognostic value using univariable Cox regression analysis. Kaplan-Meier analyses with log-rank testing was used to assess individual predictive value of calcium score, exercise-ECG and CCTA for all endpoints (ACS, PCI and CABG). Additionally, this was followed by multivariable Cox regression analysis, to assess the independent prognostic value of the different methods. Receiver-operating-characteristic (ROC) curves were plotted for four incremental models; FRS, FRS plus exercise-ECG, FRS plus exercise-ECG plus CCTA and FRS plus exercise-ECG plus CCTA plus calcium score. Area under the curve (AUC) for prediction of all endpoints by the four models was produced to evaluate incremental value of the combined approach. The same was done for every individual test. All p-values were 2 -sided, and a value below 0.05 was considered significant.

\section{RESULTS}

\section{Study population}

Of the 283 patients, $143(51)$ were male, mean age was $54 \pm 10$ years. The prevalence of a positive exercise-ECG was 102(36). Similarly, the prevalence of a positive CCTA $(\geq 50 \%)$ was $71(25)$. There were $149(53)$ patients with a zero calcium score, of which 9(6) had a significant stenosis on CCTA. There were no cases of cardiac death. CCTA was performed using 'Step and shoot' protocol in 192(68) patients, corresponding radiation dose was $3.4 \pm 1.1 \mathrm{mSv}$ (for 'Helical' protocol $9.7 \pm 3.7 \mathrm{mSv}$ ).

\section{Follow-up analysis}

During a median total follow-up of 769 days (interquartile range 644 - 1007), occurrence of ACS, PCI and CABG was recorded. ACS occurred in 6 patients at the median interim interval of 79 days (interquartile range $31-288$ ) after CCTA. Three patients met criteria for myocardial infarction (1 STEMI, 2 NSTEMI), and underwent conventional coronary angiography. Unexpectedly, in one STEMI patient no coronary lesions were identified, also previous CCTA showed no lesions. An additional 68 patients received invasive angiography, median time interval from CCTA was 64 days (interquartile range 39 -106). Of this group, 31(48) underwent invasive angiography within 60 days after CCTA, 
which was followed by a revascularization procedure in 12 patients. These cases of early revascularization were censored and not analyzed as event. Uncensored revascularization procedures were 8 PCIs and 1 CABG and occurred after a median interim interval of 83 days (interquartile range 73 - 87) after CCTA. Thus, total endpoints consisted of 15 events.

\section{Univariable analysis}

Several clinical parameters predicted for the occurrence of an event during follow-up, table 1.

Table 1. Baseline characteristics and Cox univariable analysis

\begin{tabular}{cccc}
$\begin{array}{c}\text { Event } \\
(\mathrm{n}=15)\end{array}$ & $\begin{array}{c}\text { No event } \\
(\mathrm{n}=268)\end{array}$ & HR $(95 \% \mathrm{CI})$ & P-value \\
& & & \\
\hline $55.9 \pm 7.5$ & $54.3 \pm 9.9$ & $1.02(0.97-1.07)$ & 0.52 \\
$10(67)$ & $133(50)$ & $2.00(0.68-5.86)$ & 0.21 \\
$8(53)$ & $67(25)$ & $3.29(1.19-9.08)$ & 0.02 \\
$1(7)$ & $16(6)$ & $1.10(0.15-8.39)$ & 0.92 \\
$7(47)$ & $124(46)$ & $1.01(0.37-2.80)$ & 0.98 \\
$4(27)$ & $15(6)$ & $5.56(1.77-17.46)$ & 0.003 \\
$26.9 \pm 3.4$ & $26.4 \pm 3.8$ & $1.03(0.91-1.17)$ & 0.60 \\
$147 \pm 19$ & $141 \pm 19$. & $1.02(0.99-1.05)$ & 0.18 \\
$6.0 \pm 0.9$ & $5.6 \pm 1.2$ & $1.28(0.85-1.92)$ & 0.25 \\
$30.3 \pm 19.6$ & $17.7 \pm 13.8$ & $1.04(1.02-1.07)$ & 0.001 \\
$11(73)$ & $91(34)$ & $5.14(1.64-16.13)$ & 0.005 \\
$12(80)$ & $122(46)$ & $4.59(1.30-16.28)$ & 0.02 \\
$14(93)$ & $57(21)$ & $45.82(6.02-348.5)$ & $<0.001$ \\
$0.49 \pm 0.29$ & $0.17 \pm 0.26$ & $9.66(3.26-28.60)$ & $<0.001$ \\
$4.1 \pm 2.6$ & $1.8 \pm 2.6$ & $1.24(1.08-1.41)$ & 0.002 \\
\hline
\end{tabular}

Values are presented as numbers (\%), or means \pm standard deviation. BMI, body mass index; CCTA, cardiac computed tomographic angiography; CI, confidence interval; HR, hazard ratio. Significant pvalues are in bold.

Smoking, typical chest pain symptoms and Framingham risk score were significantly associated with the occurrence of an event. In addition, a positive exercise-ECG significantly predicted for the combined endpoint [hazard ratio (HR) $5.14(95 \%$ confidence interval (CI) $1.64-16.13), \mathrm{p}=0.005]$, as well as a positive calcium score [HR 4.59 (95\% CI $1.30-16.28), \mathrm{p}=0.02$ ]. A significant stenosis on CCTA was a very strong predictor for events [HR 45.82 (95\% CI $6.02-$ 348.54), $\mathrm{p}<0.001$ ], which was higher than the involvement score [HR 1.24 
(95\% CI $1.08-1.41), \mathrm{p}=0.002]$ and segment score [HR 9.66 (95\% CI $3.26-$ 28.60), $\mathrm{p}<0.001]$.

Further, Kaplan-Meier analysis revealed that patients with a positive exercise-ECG showed significantly worse event-free survival when compared to patients with a negative exercise-ECG (Figure 1). The annual event rate for a positive exercise-ECG was $5.3 \%$, compared to $1.0 \%$ for a negative exerciseECG, p <0.01. Patients with calcium score zero had a significantly higher event-free survival when compared to a positive calcium score; annual event rate $1.0 \%$ vs. $3.9 \%$, respectively, $\mathrm{p}<0.01$ (Figure 1). Patients with a significant stenosis on CCTA showed an annual event rate of $8.7 \%$, which was significantly worse than for patients with no significant stenosis on CCTA which showed an annual event rate of $0.3 \%, \mathrm{p}<0.001$ (Figure 1). Low FRS was associated with an event rate of $0.5 \%$, intermediate FRS 2.2\% and high FRS showed an event rate of $4.7 \%, \mathrm{p}=0.04$.

Figure 1. Kaplan-Meier curves for exercise-ECG, CCTA and calcium score
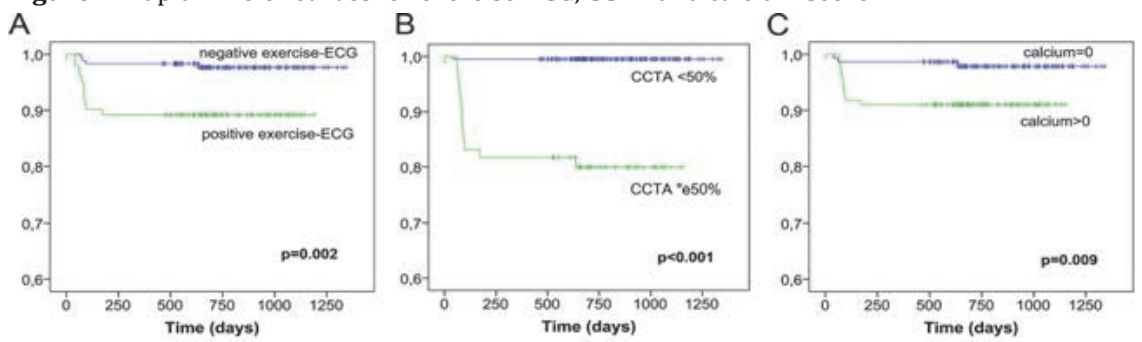

Kaplan-Meier analysis to assess the individual predictive value for the combined endpoint. A) the annual event rate for a positive exercise-ECG was $5.3 \%$, compared to $1.0 \%$ for a negative exercise-ECG, $\mathrm{p}<0.01$. B) patients with calcium score zero had an annual event rate of $1.0 \%$, a positive calcium score $3.9 \%$, p $<0.01$. C) Patients with a $\geq 50 \%$ stenosis on CCTA had an annual event rate of $8.7 \%$, which was significantly worse than for patients with no significant stenosis on CCTA which showed an annual event rate of $0.3 \%, \mathrm{p}<0.001$.

\section{Multivariable analysis}

We investigated the predictive value of the different methods using multivariable Cox regression models corrected for FRS. A positive exercise-ECG and a significant stenosis on CCTA were the only independent predictors for the occurrence of an event; [HR 3.6 (95\% CI 1.1 - 11.2), $\mathrm{p}=0.03$ ] and [HR 31.4 $(95 \%$ CI $4.0-246.6) \mathrm{p}=0.001]$ (Table 2, model 2). The addition of calcium score did not improve the model significantly and calcium score did not have independent prognostic value; [HR 0.6 (95\% CI $0.2-2.3$ ), p=0.5] (Table 2, model 3). 
Table 2. Cox multivariable analysis

\begin{tabular}{|c|c|c|c|}
\hline & & HR $(95 \%$ CI $)$ & P-value \\
\hline & FRS & $1.04(1.02-1.07)$ & 0.001 \\
\hline \multirow[t]{2}{*}{ Model 1} & FRS & $1.04(1.02-1.07)$ & 0.001 \\
\hline & Positive exercise-ECG & $4.73(1.50-14.88)$ & 0.008 \\
\hline \multirow[t]{3}{*}{ Model 2} & FRS & $1.02(0.99-1.05)$ & 0.18 \\
\hline & Positive exercise-ECG & $3.6(1.12-11.24)$ & 0.03 \\
\hline & Significant lesion CCTA $(\geq 50 \%)$ & $31.44(4.01-246.64)$ & 0.001 \\
\hline \multirow[t]{4}{*}{ Model 3} & FRS & $1.02(0.99-1.05)$ & 0.16 \\
\hline & Positive exercise-ECG & $3.64(1.15-11.55)$ & 0.03 \\
\hline & Significant lesion CCTA $(\geq 50 \%)$ & $40.34(4.61-353.05)$ & 0.001 \\
\hline & Calcium score $>0$ & $0.59(0.15-2.29)$ & 0.45 \\
\hline
\end{tabular}

FRS, Framingham risk score; CCTA, cardiac computed tomographic angiography; CI, confidence interval; HR, hazard ratio. Model 1: prognostic value of exercise-ECG adjusted for FRS. Model 2:prognostic value CCTA, adjusted for exercise-ECG and FRS. Model 3: prognostic value of a positive calcium score adjusted for CCTA, exercise-ECG and FRS. Significant p-values are in bold.

Figure 2. ROC analysis

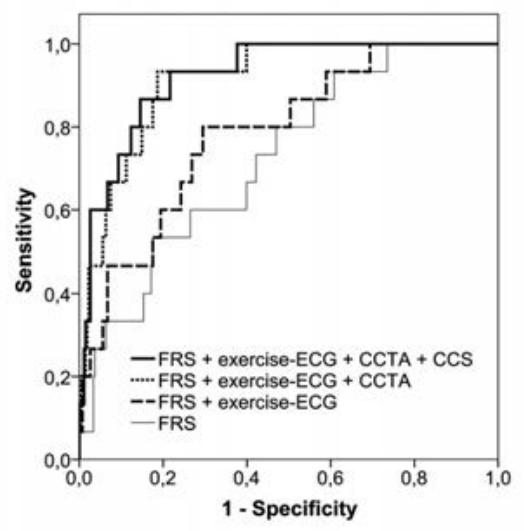

ROC-curves of four incremental models predicting for cardiovascular events. FRS (thin line, AUC 0.72 (95\% confidence interval 0.60 $0.85)$ ), together with exercise-ECG (striped line, AUC 0.79 (95\% confidence interval 0.67 0.90)), together with both exercise-ECG and CCTA (dotted line, AUC 0.91 (95\% confidence interval 0.86 - 0.97)) and combined FRS, exercise-ECG, CCTA and calcium score (thick line, AUC 0.92 (95\% confidence interval 0.87 0.98)), to assess combined predictive value for the combined endpoint.

\section{Combined predictive value}

FRS, exercise-ECG, CCTA and calcium score were studied for combined predictive value for all endpoints, using receiver operating characteristics (ROC) analysis (Figure 2). AUC for FRS to predict for all events was 0.72 (95\% CI $0.60-0.85)$, which increased to $0.79(95 \%$ CI $0.67-0.90 ; \mathrm{p}=0.26)$, when exercise-ECG was added. The AUC of the combined use of FRS, exercise-ECG and CCTA was 0.91 (95\% CI $0.86-0.97$; $\mathrm{p}=0.006)$. No significant improvement of the model was achieved when calcium score was added to the model; AUC 0.92 (95\% CI 0.87 - 0.98; p=0.11). 
In perspective, AUC for exercise-ECG alone was 0.70 (95\% CI 0.56 $0.83)$, for CCTA alone 0.86 (95\% CI 0.78 - 0.94) and for calcium score alone 0.69 (95\% CI $0.56-0.82)$.

\section{DISCUSSION}

Our study suggests that the sequential use of exercise-ECG and CCTA provided high prognostic yield for cardiovascular events, with a high AUC of 0.91. In patients with an intermediate pre-test probability for CAD presenting with stable chest pain, CCTA provided evident additional prognostic value over clinical risk profiling and exercise-ECG. Still, a positive exercise-ECG remained an independent predictor for the combined endpoint

For most clinicians, assessment of traditional risk factors will be the first step in the diagnostic work-up of chest pain patients. In addition, exercise-ECG is widely used to provide further evaluation of patients presenting with chest pain, although the proportion of inconclusive tests is relatively high $(22 \%$ in our population) and the sensitivity, negative predictive value and prognostic abilities low. ${ }^{1,22}$ Despite this, the technique might provide other prognostic parameters, such as exercise tolerance and ST/HR hysteresis. ${ }^{23}$ Our results indicate that in an intermediate pre-test probability population, the prognostic performance of exercise-ECG is only moderate, although it provided additional prognostic information independent from CCTA. Therefore, the sequential use of exercise-ECG and CCTA provided high prognostic yield.

There has been a great interest in calcium score as a risk stratification tool, mainly in asymptomatic patients. ${ }^{24,25}$ The technique is attractive, as it provides a fast measurement of presence and severity of CAD, with a minimal radiation dose and no use of contrast medium. However, especially in symptomatic patients, a zero calcium score does not rule out CAD. In our population $6 \%$ of patients presenting with zero calcium score had a significant lesion on CCTA, which is comparable to previous reports. ${ }^{26,27}$ Other reports indicate that patients with a zero calcium score have a low prevalence of inducible ischemia on PET/CT, but prevalence was still about 1\%.28 Although low, the annual event rate in patients with a zero calcium score in our population was $1 \%$. Further, we showed that calcium score did not independently predict for the combined endpoint of all events in this patient group. There are several considerations that might explain the shortcoming of calcium scoring in this patient group. For instance, calcium score does not take into account presence of noncalcified plaques, while these seem to be more vulnerable to rupture, conse- 
quently posing greater risk on a cardiovascular events. ${ }^{6}$ Because of the continuous advances in CCTA, its radiation dose is approximating that of calcium score. This instigates a paradigm shift, because when calcium score seems to have no additive prognostic value to CCTA, it still increases radiation exposure. Our findings are in agreement with Kwon et al. who suggested that calcium score may no longer be incorporated in CCTA protocols, in terms of determining prognosis. ${ }^{29}$

Our data support the prevailing notion that a $\geq 50 \%$ stenosis as found on CCTA is an effective cut-off value for the prediction of cardiovascular endpoints. ${ }^{20,30}$ Also the observation that segment score as well as involvement score are associated with events is in compliance with other reports. ${ }^{20}$ Additionally, there is increasing evidence that the use of CCTA is likely to allow the visualization of the vessel wall itself, plaque morphology and the extent of outward remodeling, and therefore the prognostic importance of the coronary lesion. ${ }^{6}$

Exceptionally, one patient in our cohort without CAD on CCTA developed ACS. This 59-year old female, in which CCTA as well as invasive angiography revealed no CAD, presented with STEMI during follow-up. The best we could explain this is by coronary erosion, stress-related myocardial involvement, coronary spasm or an obscure source of embolization. We have this patient in close follow-up and may learn more in the coming years.

Since CCTA offers evaluation of both luminal stenosis and plaque morphology, and exercise-ECG may provide the functional importance of the obstructive lesion, this combination proposes to be both informative and cost saving. Currently, most guidelines mention the use of stress nuclear perfusion imaging or stress echocardiography for further work-up in patients presenting with chest pain.2,3,4 Although nuclear perfusion imaging is very well-established, its sensitivity and specificity are still suboptimal, radiation doses comparable to CCTA. On the other hand, the use of stress echocardiography has the disadvantage of being operator dependent, and the sensitivity and specificity are not optimal. The decrease in radiation dose achieved by the latest CCTA scanners, the patient friendliness of the technique and excellent information for the coronary anatomy could make CCTA a test of choice. ${ }^{31}$ Although it is becoming possible to obtain some information about myocardial perfusion using newer generation CT scanners, the addition of exercise-ECG may compensate for the inadequacy of CCTA. We provide evidence that this new approach seems to provide a high prognostic yield. 
Our data show some unique features but due to small sample size may at best be considered hypothesis generating. It is known that anatomic lesions do not correlate well neither with functional importance of a lesion nor the likelihood of acute events, and the objective evidence of ischemia and the plaque morphology is mandatory. ${ }^{8}$ It is therefore conceivable that in this study the combined use of exercise-ECG and CCTA provided an attractive prognostic value for predicting all kinds of cardiovascular events.

\section{Study limitations}

Due to the retrospective nature of this study, the application of both exerciseECG and CCTA were left to the discretion of the cardiologist, possibly implicating referral bias. It is possible that referral bias might have negatively influenced the overall predictive value of exercise-ECG. Revascularization in general will be partly driven by a positive CCTA. Therefore we censored all revascularization procedures within 60 days after CCTA, to minimize this bias. The event rate and follow-up period of this study were relatively low, although comparable to other studies and as expected in an intermediate risk population. Events were followed-up in the electronic database of the Maastricht University Medical Center. As all patients were followed-up in our clinic, we expect the completeness of the follow-up to be high.

\section{Conclusion}

In conclusion, our data suggest that the subsequent use of exercise-ECG for functional testing and CCTA for coronary anatomy visualization provides a high diagnostic yield. In a stable chest pain population with an intermediate pre-test probability for CAD, CCTA is a good choice as a follow-up diagnostic test and provides essential information as to whether individual patients will develop a cardiovascular endpoint. 


\section{References}

1. Gibbons RJ, Balady GJ, Bricker JT, et al. ACC/AHA 2002 guideline update for exercise testing: summary article: a report of the American College of Cardiology/American Heart Association Task Force on Practice Guidelines (Committee to Update the 1997 Exercise Testing Guidelines). Circulation 2002;106:1883-92.

2. Douglas PS, Khandheria B, Stainback RF, et al. ACCF/ASE/ACEP/AHA/ASNC/SCAI/SCCT/SCMR 2008 appropriateness criteria for stress echocardiography: a report of the American College of Cardiology Foundation Appropriateness Criteria Task Force, American Society of Echocardiography, American College of Emergency Physicians, American Heart Association, American Society of Nuclear Cardiology, Society for Cardiovascular Angiography and Interventions, Society of Cardiovascular Computed Tomography, and Society for Cardiovascular Magnetic Resonance: endorsed by the Heart Rhythm Society and the Society of Critical Care Medicine. Circulation 2008;117:1478-97.

3. Hendel RC, Berman DS, Di Carli MF, et al. ACCF/ASNC/ACR/AHA/ASE/SCCT/SCMR/SNM 2009 appropriate use criteria for cardiac radionuclide imaging: a report of the American College of Cardiology Foundation Appropriate Use Criteria Task Force, the American Society of Nuclear Cardiology, the American College of Radiology, the American Heart Association, the American Society of Echocardiography, the Society of Cardiovascular Computed Tomography, the Society for Cardiovascular Magnetic Resonance, and the Society of Nuclear Medicine. Circulation 2009;119:e561-87.

4. Fox K, Garcia MA, Ardissino D, et al. Guidelines on the management of stable angina pectoris: executive summary: The Task Force on the Management of Stable Angina Pectoris of the European Society of Cardiology. Eur Heart J 2006;27:1341-81.

5. Budoff MJ, Dowe D, Jollis JG, et al. Diagnostic performance of 64-multidetector row coronary computed tomographic angiography for evaluation of coronary artery stenosis in individuals without known coronary artery disease: results from the prospective multicenter ACCURACY (Assessment by Coronary Computed Tomographic Angiography of Individuals Undergoing Invasive Coronary Angiography) trial. J Am Coll Cardiol 2008;52:1724-32.

6. Motoyama S, Sarai M, Harigaya H, et al. Computed tomographic angiography characteristics of atherosclerotic plaques subsequently resulting in acute coronary syndrome. J Am Coll Cardiol 2009;54:49-57.

7. Cury RC, Nieman K, Shapiro MD, et al. Comprehensive assessment of myocardial perfusion defects, regional wall motion, and left ventricular function by using 64-section multidetector CT. Radiology 2008;248:466-75.

8. Meijboom WB, Van Mieghem CA, van Pelt N, et al. Comprehensive assessment of coronary artery stenoses: computed tomography coronary angiography versus conventional coronary angiography and correlation with fractional flow reserve in patients with stable angina. J Am Coll Cardiol 2008;52:636-43.

9. Diamond GA, Forrester JS. Analysis of probability as an aid in the clinical diagnosis of coronary-artery disease. N Engl J Med 1979;300:1350-8. 
10. Taylor AJ, Cerqueira M, Hodgson JM, et al. ACCF/SCCT/ACR/AHA/ASE/ASNC/NASCI/SCAI/SCMR 2010 Appropriate Use Criteria for Cardiac Computed Tomography. A Report of the American College of Cardiology Foundation Appropriate Use Criteria Task Force, the Society of Cardiovascular Computed Tomography, the American College of Radiology, the American Heart Association, the American Society of Echocardiography, the American Society of Nuclear Cardiology, the North American Society for Cardiovascular Imaging, the Society for Cardiovascular Angiography and Interventions, and the Society for Cardiovascular Magnetic Resonance. J Cardiovasc Comput Tomogr 2010;4:407 e1-33.

11. Shewan LG, Coats AJS. Ethics in the authorship and publishing of scientific articles. International Journal of Cardiology 2010;144:1-2.

12. D'Agostino RB, Sr., Vasan RS, Pencina MJ, et al. General cardiovascular risk profile for use in primary care: the Framingham Heart Study. Circulation 2008;117:743-53.

13. Fletcher GF, Balady GJ, Amsterdam EA, et al. Exercise standards for testing and training: a statement for healthcare professionals from the American Heart Association. Circulation 2001;104:1694-740.

14. Pontone G, Andreini D, Bartorelli AL, et al. Diagnostic accuracy of coronary computed tomography angiography: a comparison between prospective and retrospective electrocardiogram triggering. J Am Coll Cardiol 2009;54:346-55.

15. Hausleiter J, Meyer T, Hadamitzky M, et al. Radiation dose estimates from cardiac multislice computed tomography in daily practice: impact of different scanning protocols on effective dose estimates. Circulation 2006;113:1305-10.

16. Agatston AS, Janowitz WR, Hildner FJ, et al. Quantification of coronary artery calcium using ultrafast computed tomography. J Am Coll Cardiol 1990;15:827-32.

17. Laufer EM, Mingels AM, Winkens MH, et al. The extent of coronary atherosclerosis is associated with increasing circulating levels of high sensitive cardiac troponin T. Arterioscler Thromb Vasc Biol 2010;30:1269-75.

18. Austen WG, Edwards JE, Frye RL, et al. A reporting system on patients evaluated for coronary artery disease. Report of the Ad Hoc Committee for Grading of Coronary Artery Disease, Council on Cardiovascular Surgery, American Heart Association. Circulation 1975;51:5-40.

19. Raff GL, Abidov A, Achenbach S, et al. SCCT guidelines for the interpretation and reporting of coronary computed tomographic angiography. J Cardiovasc Comput Tomogr 2009;3:122-36.

20. Min JK, Shaw LJ, Devereux RB, et al. Prognostic value of multidetector coronary computed tomographic angiography for prediction of all-cause mortality. J Am Coll Cardiol 2007;50:1161-70. 
21. Braunwald E, Antman EM, Beasley JW, et al. ACC/AHA 2002 guideline update for the management of patients with unstable angina and non-ST-segment elevation myocardial infarction-summary article: a report of the American College of Cardiology/American Heart Association task force on practice guidelines (Committee on the Management of Patients With Unstable Angina). J Am Coll Cardiol 2002;40:1366-74.

22. Maffei E, Seitun S, Martini C, et al. CT coronary angiography and exercise ECG in a population with chest pain and low-to-intermediate pre-test likelihood of coronary artery disease. Heart 2010;96:1973-9.

23. Kronander H, Hammar N, Fischer-Colbrie W, et al. Analysis of ST/HR hysteresis improves long-term prognostic value of exercise ECG test. Int J Cardiol 2011;148:64-9.

24. Vliegenthart R, Oudkerk M, Hofman A, et al. Coronary calcification improves cardiovascular risk prediction in the elderly. Circulation 2005;112:572-7.

25. Arad Y, Spadaro LA, Goodman K, et al. Prediction of coronary events with electron beam computed tomography. J Am Coll Cardiol 2000;36:1253-60.

26. Sosnowski M, Pysz P, Szymanski L, et al. Negative calcium score and the presence of obstructive coronary lesions in patients with intermediate CAD probability. Int J Cardiol 2011;148:e16-8.

27. Morita H, Fujimoto S, Kondo T, et al. Prevalence of computed tomographic angiographyverified high-risk plaques and significant luminal stenosis in patients with zero coronary calcium score. Int J Cardiol 2012;158:272-8.

28. Esteves FP, Khan A, Correia LC, et al. Absent coronary artery calcium excludes inducible myocardial ischemia on computed tomography/positron emission tomography. Int J Cardiol 2011;147:424-7.

29. Kwon SW, Kim YJ, Shim J, et al. Coronary artery calcium scoring does not add prognostic value to standard 64-section CT angiography protocol in low-risk patients suspected of having coronary artery disease. Radiology 2011;259:92-9.

30. Reimann AJ, Tsiflikas I, Pecha S, et al. Prognostic value of significant and non-significant coronary artery stenosis detection using MDCT for major adverse cardiac events. Int J Cardiol 2011;149:126-8.

31. Achenbach S, Daniel WG. Cardiac imaging in the patient with chest pain: coronary CT angiography. Heart 2010;96:1241-6. 



\section{Chapter}

\section{Gender Difference in Prognostic Value of Cardiac Computed Tomographic Angiography?}

Mathijs O. Versteylen, Ivo A. Joosen, Bas L. Kietselaer, Joachim E. Wildberger, Harry J. Crijns, Leonard Hofstra

Accepted for publication in a different format in JACC Cardiovasc Imaging 


\section{ABSTRACT}

Background The pathophysiology of acute coronary events in men and women differs. In contrast to men, coronary events in women more often occur in the absence of significant atherosclerosis. Therefore, we investigated the potential gender difference in prognostic value of cardiac computed tomographic angiography (CCTA).

Methods 1,210 stable chest pain patients underwent CCTA and were followedup for a mean of $1,166 \pm 259$ days. CCTA was performed using a 64 -slice scanner. Obstructive CAD was defined as $\geq 50 \%$ luminal stenosis. Patients were followed-up for a combined endpoint (cardiac death, unstable angina, myocardial infarction and late revascularization [>60 days after CCTA]). Prognostic value was assessed using Cox regression analysis, differences in event rates by Fisher's exact test.

Results A total of 60 events occurred ( 3 cardiac death, 12 myocardial infarction, 7 unstable angina, 38 late revascularization). Obstructive CAD predicted significantly for the combined endpoint in both men (hazard ratio (HR) 37.2 [95\% confidence interval; 8.9-154.3], $\mathrm{p}<0.001$ ) and women (HR 18.4 [95\% confidence interval; 6.2-54.4], $\mathrm{p}<0.001$. However, out of the events in men, 2/38 (5.3\%) occurred in patients without previous obstructive CAD, compared with $4 / 22(18.2 \%)$ in women, $p=0.11$. Moreover, $3 / 22$ (13.6\%) of women suffering an event had a previously normal CCTA, against $0 \%$ in men, $\mathrm{p}<0.05$.

Conclusions The presence of obstructive CAD on CCTA is a strong predictor for the occurrence of a coronary event, in both men and women. However, in women with previously non-obstructive or even normal CCTA findings, events are not uncommon. 


\section{INTRODUCTION}

Evidence on the prognostic value of cardiac computed tomographic angiography (CCTA) in stable chest pain patients is emerging. Several studies concluded that the presence of a severe coronary artery obstruction visible on CCTA is a strong independent predictor for short-term cardiovascular events ${ }^{1-5}$ and all-cause mortality.6,7 In addition, also the presence of non-obstructive coronary artery disease (CAD) has been associated with an increased risk for all-cause mortality. ${ }^{8}$ On the other hand, patients without any CAD visible on CCTA are reported to be at very low risk for major adverse cardiovascular events.

Although obstructive CAD has strong predictive power, acute coronary syndrome (ACS) often occurs in previously non-obstructive angiographic lesions. ${ }^{9}$ This is especially apparent in women, as females presenting with ACS tend to have less extensive CAD and less obstructive lesions as compared with men. ${ }^{10}$ Additionally, it is widely accepted that a substantial part of ACS is not caused by coronary plaque rupture, but by erosion of relatively mild coronary plaques. ${ }^{11}$ This mechanism is a substantially more frequent cause of ACS in younger females. ${ }^{12-14}$ This obvious gender difference in pathological mechanisms of coronary events can potentially limit the prognostic value of CCTA. However, up to now most studies did not assess the gender difference in prognostic value of CCTA.

Since the pathophysiological mechanism of coronary events in men and women differs, we hypothesized that the prognostic value of CCTA is lower in women as compared with men. Therefore, the goal of this study was to investigate the ability of CCTA to predict coronary events, in both men and women with stable chest pain.

\section{METHODS}

\section{Study population}

We prospectively included consecutive stable chest pain patients referred for CCTA from within the Maastricht University Medical Center, from June 2007 until June 2010, into this observational study. Hospital protocol precluded CCTA when calcium score was $>1000$. CCTA was also not performed in patients with unstable angina, hemodynamic instability, pregnancy, renal insufficiency and severe iodine allergy. Excluded were patients with previously rec- 
orded CAD (myocardial infarction, acute coronary syndrome, revascularization procedure) and non-diagnostic CCTA. From 1,487 patients who underwent both calcium score as well as CCTA, 89 had a history of CAD, 125 had a nondiagnostic CCTA and in 63 no clinical data was available, eventually 1,210 subjects were subject of this study. All patients signed informed consent prior to inclusion in the study. The study protocol conforms to the ethical guidelines of the 1975 Declaration of Helsinki as reflected in a priori approval by the institution's human research committee. Risk factors were assessed prior to CCTA and Framingham risk score (FRS) was calculated to estimate the 10-year risk of cardiovascular disease. ${ }^{15}$

\section{CCTA acquisition and assessment}

CCTA was performed using a 64-slice multi detector computed tomography scanner (Brilliance 64; Philips Healthcare, Best, The Netherlands) with a $64 \mathrm{x}$ $0.625 \mathrm{~mm}$ slice collimation, gantry rotation time of $420 \mathrm{~ms}$, a tube voltage of 80 or $120 \mathrm{kV}$ and the tube current varied from 150 to $1000 \mathrm{mAs}$, depending on the patient's weight. Patients received 5-20 mg of Metoprolol intravenously to lower the heart rate to $<65 \mathrm{bpm}$ as well as $0.8 \mathrm{mg}$ sublingual nitroglycerin spray. A native scan using $120 \mathrm{kV}$ and $3 \mathrm{~mm}$ slice thickness was performed to determine the calcium score according to the Agatston method. ${ }^{16}$ Patients received a contrast bolus (Xenetix 350, Guerbet) of $85-110 \mathrm{ml}$, injected at a rate of $5.0-7.2 \mathrm{ml} / \mathrm{s}$. In patients with heart rate $<65 \mathrm{bpm}$, a prospective ECGtriggered axial scan was performed, in patients with heart rate $>65 \mathrm{bpm}$, a retrospective ECG-triggered spiral acquisition protocol was used. The CCTA protocol was described in more detail previously. ${ }^{17}$ Mean total estimated radiation dose was calculated by multiplying the dose-length product by the conversion factor of $0.014 \mathrm{mSv} / \mathrm{mGy} / \mathrm{cm}$ for the thorax. ${ }^{18}$

Using the American Heart Association in 16 coronary segments model, CT angiograms were independently analyzed by a cardiologist and radiologist who were both experienced in reading CCTA and blinded to clinical information. In case of disagreement, consensus was reached by reviewing the images jointly. The coronary artery tree was assessed using the source images on the provided software (Cardiac Comprehensive Analysis, Philips Healthcare), and CAD was reported for every segment as no CAD (no lesion, score 0), nonobstructive CAD (lesion with diameter stenosis of $<50 \%$, score 1 ), or obstructive CAD (lesion with diameter stenosis $\geq 50 \%$, score 3). ${ }^{19}$ Overall obstructive $\mathrm{CAD}$ was defined as having one or more segments with $\geq 50 \%$ luminal stenosis. 
In addition, a segment stenosis score was the sum of the lesion severity score in all 16 segments (range $0-48$ ). An involvement score was calculated by counting all diseased vessel segments (irrespective of degree of stenosis), and the total score ranged from $0-16 .{ }^{6}$ Plaques were categorized as calcified (exclusively content with density $>130$ Hounsfield units), non-calcified (exclusively content with density $<130$ Hounsfield units), or mixed (characteristics of both calcified and non-calcified plaques).

\section{Follow-up}

Electronic patient records were monitored for the combined endpoint of cardiovascular death, myocardial infarction, unstable angina and late revascularization. Late revascularization was defined as a percutaneous coronary intervention (PCI) or coronary artery bypass graft $(\mathrm{CABG})$ procedure $>60$ days after CCTA. Additionally, the national mortality records were checked. Myocardial infarction was defined as a rise and/or fall in troponin $\mathrm{T}$, with at least one value above the 99 th percentile $(0.01 \mu \mathrm{g} / \mathrm{L})$, combined with either typical chest pain, ECG changes suggestive for ischemia, development of pathological Q-waves, or imaging evidence of loss of myocardium or regional wall motion abnormality. ${ }^{20}$ Unstable angina was defined as typical angina pectoris requiring hospitalization, accompanied by ST-segment elevation/depression of $\geq 1 \mathrm{~mm}$ and/or invasive angiographic conformation of a culprit lesion. ${ }^{21}$ Revascularizations within 60 days of CCTA were censored at the time of procedure, as these would be predominantly CCTA driven. Further, we censored follow-up after the first endpoint, so that the recorded ACS was not a complication of revascularization therapy.

\section{Statistical methods}

Data were analyzed using SPSS 19.0 (IBM, SPSS, Chicago, IL). Continuous variables were reported as means \pm standard deviations $(\mathrm{SD})$, or median and interquartile range (IQR) for parameters that were not normally distributed. Normality of data distribution was evaluated using the Kolmogorov-Smirnov test and plot histograms. Proportions (\%) were used for categorical values. Fisher's exact test or chi-square test was used to measure differences between categorical values, depending on expected count. For continuous variables, independent t-test or Mann-Whitney test was performed to assess difference between values, depending on normality of distribution. Kaplan-Meier analyses 
with log-rank testing was used to assess individual predictive value of CCTA. Additionally, this was followed by Cox regression analysis. Multivariable Cox regression analysis using forward stepwise selection was performed to assess prognostic value of CCTA when corrected for FRS and calcium score. All pvalues were 2 -sided, and a value below 0.05 was considered significant.

\section{RESULTS}

\section{Study population}

The study population consisted of $640(53 \%)$ men and $570(47 \%)$ women, mean age was $57 \pm 11$ years. Overall, women were older, had a lower body mass index (BMI), higher total cholesterol level and more often a positive family history and typical chest pain, table 1 . The pre-test probability for having CAD was $42 \pm 12 \%$ for men and $34 \pm 27 \%$ for women, $\mathrm{p}<0.001$. Overall clinical risk profile resulted in a mean FRS of $24 \pm 16$ for men and $15 \pm 11$ for women, $\mathrm{p}<0.001$. A prospective ECG-gated CCTA protocol was used in $66 \%$ of the population. The overall estimated radiation dose was $5.5 \pm 4.1 \mathrm{mSv}$.

Table 1. Baseline characteristics and difference between men and women

\begin{tabular}{|c|c|c|c|c|}
\hline & $\begin{array}{c}\text { All } \\
(\mathrm{n}=1,210)\end{array}$ & $\begin{array}{c}\text { Men } \\
(\mathrm{n}=640)\end{array}$ & $\begin{array}{l}\text { Women } \\
(n=570)\end{array}$ & P-value \\
\hline Age (years) & $57 \pm 11$ & $56 \pm 11$ & $58 \pm 11$ & $<0.001$ \\
\hline Smoking & $285(24 \%)$ & $142(22 \%)$ & $143(25 \%)$ & 0.28 \\
\hline Diabetes & $105(9 \%)$ & $53(8 \%)$ & $52(9 \%)$ & 0.64 \\
\hline Positive family history & $459(38 \%)$ & $213(33 \%)$ & $246(43 \%)$ & $<0.001$ \\
\hline Typical chest pain & $144(12 \%)$ & $59(9 \%)$ & $85(15 \%)$ & 0.002 \\
\hline BMI $\left(\mathrm{kg} / \mathrm{m}^{2}\right)$ & $27 \pm 4$ & $27 \pm 4$ & $26 \pm 5$ & 0.004 \\
\hline Systolic blood pressure (mmHg) & $142 \pm 19$ & $142 \pm 18$ & $143 \pm 20$ & 0.24 \\
\hline Total cholesterol (mmol/L) & $5.4 \pm 1.2$ & $5.4 \pm 1.2$ & $5.5 \pm 1.2$ & 0.02 \\
\hline HDL cholesterol (mmol/L) & $1.3 \pm 0.8$ & $1.2 \pm 0.9$ & $1.5 \pm 0.5$ & $<0.001$ \\
\hline LDL cholesterol (mmol/L) & $3.4 \pm 1.1$ & $3.4 \pm 1.1$ & $3.4 \pm 1.1$ & 0.79 \\
\hline Triglycerides (mmol/L) & $1.76 \pm 1.30$ & $1.91 \pm 1.54$ & $1.59 \pm 0.93$ & $<0.001$ \\
\hline Statin use & $442(37 \%)$ & $220(34 \%)$ & $222(39 \%)$ & 0.10 \\
\hline Antihypertensive agent use & $668(55 \%)$ & $350(55 \%)$ & $318(56 \%)$ & 0.70 \\
\hline Framingham risk score & $20 \pm 15$ & $24 \pm 16$ & $15 \pm 11$ & $<0.001$ \\
\hline
\end{tabular}

Values are presented as numbers (\%), means (standard deviation). BMI = body mass index; CAD = coronary artery disease; CCTA = cardiac computed tomographic angiography; HDL = high-density lipoprotein; LDL = low-density lipoprotein. 


\section{Plaque burden}

Men showed higher calcium score, more segments involved with atherosclerosis and a higher proportion of patient with any or obstructive CAD (table 2). Differences in plaque morphology between men and women were investigated on a per plaque level. Women had a significantly larger proportion of noncalcified plaques and less calcified plaques as compared with men $(\mathrm{p}<0.001)$.

Table 2. CCTA characteristics and difference between men and women

\begin{tabular}{lcccc} 
& $\begin{array}{c}\text { All } \\
(\mathrm{n}=1,210)\end{array}$ & $\begin{array}{c}\text { Men } \\
(\mathrm{n}=640)\end{array}$ & $\begin{array}{c}\text { Women } \\
(\mathrm{n}=570)\end{array}$ & P-value \\
\hline Calcium score & $7(0-109)$ & $19(0-164)$ & $0(0-63)$ & $<0.001$ \\
Any CAD & $767(63 \%)$ & $448(70 \%)$ & $319(56 \%)$ & $<0.001$ \\
Obstructive CAD & $336(28 \%)$ & $218(34 \%)$ & $118(21 \%)$ & $<0.001$ \\
Involvement score & $1(0-4)$ & $2(0-5)$ & $1(0-3)$ & $<0.001$ \\
Left main stenosis ( $\geq 50 \%)$ & $30(2.5 \%)$ & $23(3.6 \%)$ & $7(1.2 \%)$ & 0.008 \\
Per-plaque analysis & & & & \\
Total number of plaques & 2,797 & 1,872 & 925 & \\
Non-calcified plaques & $419(15 \%)$ & $243(13 \%)$ & $176(19 \%)$ & $<0.001$ \\
Mixed plaques & $894(32 \%)$ & $619(33 \%)$ & $275(30 \%)$ & 0.08 \\
Calcified plaques & $1,484(53 \%)$ & $1,010(54 \%)$ & $474(51 \%)$ & $<0.001$ \\
\hline
\end{tabular}

Values are presented as numbers (\%) or median (interquartile range). BMI = body mass index; CAD = coronary artery disease; $\mathrm{CCTA}=$ cardiac computed tomographic angiography; HDL = high-density lipoprotein; LDL = low-density lipoprotein.

\section{Follow-up}

Patients were followed up for a mean 1,166 \pm 259 days. The follow-up time for men and women did not differ: 1,178 \pm 258 days vs. 1,153 \pm 259 days, respectively, $\mathrm{p}=0.09$. A total of 3 cardiac deaths, 12 myocardial infarctions, 7 unstable angina cases and 38 late revascularizations were reported. The combined endpoint consisted of these 60 coronary endpoints, of which 38 occurred in men ( 2 cardiac death, 6 myocardial infarction, 3 unstable angina, 27 late revascularization) and 22 in women ( 1 cardiac death, 6 myocardial infarction, 4 unstable angina, 11 late revascularization), $\mathrm{p}=0.11$. In addition, 8 non-cardiac deaths were reported, which were not used as an endpoint (4 malignancy, 1 respiratory insufficiency, 1 renal insufficiency, 1 sepsis, 1 unknown), table 3. 
Table 3. Distribution of events during follow-up

\begin{tabular}{|c|c|c|c|c|}
\hline & $\begin{array}{c}\text { All } \\
(n=1,210)\end{array}$ & $\begin{array}{c}\text { Men } \\
(n=640)\end{array}$ & $\begin{array}{l}\text { Women } \\
(n=570)\end{array}$ & P-value \\
\hline Combined endpoint & 60 & $38(5.9 \%)$ & $22(3.9 \%)$ & 0.11 \\
\hline Cardiac death & 3 & $2(0.3 \%)$ & $1(0.2 \%)$ & 1.00 \\
\hline Nonfatal myocardial infarction & 12 & $6(0.9 \%)$ & $6(1.1 \%)$ & 1.00 \\
\hline Unstable angina & 7 & $3(0.5 \%)$ & $4(0.7 \%)$ & 0.71 \\
\hline Late revascularization & 38 & $27(4.2 \%)$ & $11(1.9 \%)$ & 0.03 \\
\hline Non-cardiac death & 8 & $6(0.9 \%)$ & $2(0.4 \%)$ & 0.29 \\
\hline $\begin{array}{l}\text { Combined endpoint obstructive CAD } \\
(n=336)\end{array}$ & 54 & $36(16.5 \%)$ & $18(15.3 \%)$ & 0.88 \\
\hline $\begin{array}{l}\text { Combined endpoint non-obstructive CAD } \\
(n=874)\end{array}$ & 6 & $2(0.5 \%)$ & $4(0.9 \%)$ & 0.69 \\
\hline
\end{tabular}

Values are presented as numbers (\%). CAD = coronary artery disease

\section{Narrative of patients without previous CAD who developed an event}

1. Woman, 47 years old, no diabetes, no hypertension, active smoker (30 pack years), indication CCTA: typical chest pain, medication: Metoprolol $50 \mathrm{mg}$, LDL-cholesterol: 1.9, FRS: 9. Later presents with typical chest pain, maximal troponin T 2.41, ECG showed lateral ST-elevation. Coronary angiography revealed a $100 \%$ blunt occlusion in the large, tortuous diagonal branch supplying the free wall to apex region (figure 1). Echocardiography showed an akinetic apex. Diagnosis was acute lateral infarction, medical treatment.

2. Woman, 68 years old, no diabetes, no hypertension, stopped smoking (15 pack years), indication CT: typical chest pain, medication: Simvastatin $20 \mathrm{mg}$, LDL-cholesterol: 2.9, FRS: 9. Later presents with typical chest pain, maximal troponin T 0.04, normal ECG. Coronary angiography was normal. Diagnosis was unspecified myocardial infarction, medical treatment.

3. Woman, 59 years old, no diabetes, no hypertension, no smoking. Indication CT: typical chest pain, medication: no, LDL-cholesterol: 3.0, FRS: 9. Later presents with typical chest pain and secondary witnessed ventricular fibrillation. ECG showed ST-elevation in the inferior, posterior and lateral region. Maximal troponin $\mathrm{T}$ was 1.24. Coronary angiography revealed a hypokinetic apex, but no CAD. Thallium scan showed lateral infarction and ischemia in the apical, anterior and inferior region. Diagnosis was acute myocardial infarction in the inferior, posterior and lateral region, medical treatment. 
Figure 1. Patient presenting with myocardial infarction after previous normal CCTA

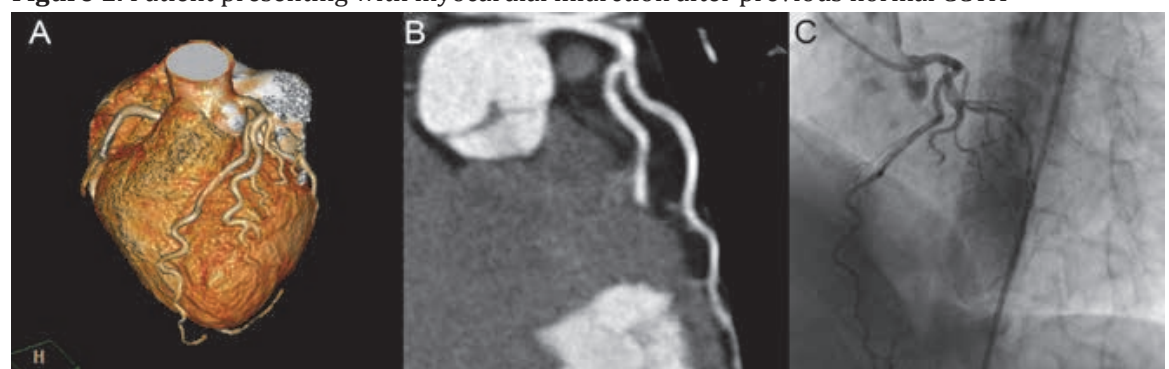

Example of a 47-year old women presenting with acute lateral myocardial infarction. A. Volume rendered image of CCTA showing LAD and diagonal anatomy. B. Stretched multiplanar reconstruction showing normal diagonal branch C. Subsequently, the patient presented with acute myocardial infarction and conventional angiography revealed an obstructed diagonal branch.

\section{Prediction of events}

In table 4 , the association of parameters with the combined endpoint is described. Next to several important clinical parameters, a higher calcium score, a higher number of plaques and presence of any or obstructive CAD were important predictors for the combined endpoint. Further, presence of plaques with any non calcified or mixed content predicted for the occurrence of events. On the other hand, no hard coronary events occurred in patients with solely calcified coronary plaques, i.e. all patients with a hard event had at least one mixed or non-calcified plaque.

Both in men and women, the presence of obstructive CAD predicted significantly for the occurrence of the combined endpoint. The annualized event rate for men with obstructive CAD was $5.12 \%$, compared with $0.15 \%$ for men with non-obstructive CAD, $\mathrm{p}<0.001$. For women with obstructive CAD the annualized event rate was $4.83 \%$, compared with $0.28 \%$ for women with non-obstructive CAD, $\mathrm{p}<0.001$. Further, out of the events in men, $2 / 38(5.3 \%)$ occurred in patients without previous obstructive CAD, compared with 4/22 $(18.2 \%)$ in women, $\mathrm{p}=0.11$. Figure 2 shows an example of a female patient without obstructive CAD who develops a myocardial infarction during followup. Moreover, $3 / 22(13.6 \%)$ of women suffering an event had a previously normal CCTA, against $0 \%$ in men, $\mathrm{p}<0.05$. Importantly, all these events were myocardial infarctions, except for one man who underwent PCI. 
Figure 2. Patient with myocardial infarction after previous non-obstructive CAD

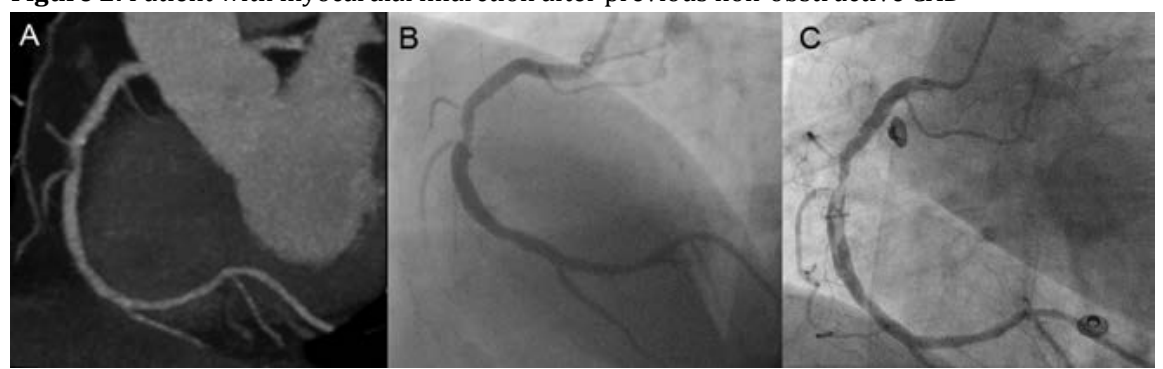

Example of a 51-year old woman presenting with a myocardial infarction after a previous CCTA revealed non-obstructive CAD. A: CCTA revealed a non-obstructive non-calcified plaque in the RCA mid segment. B: Conventional coronary angiography confirmed the lesion. C: Subsequently, the patient presented with an acute myocardial infarction, conventional coronary angiography revealed a large thrombus at the site of the plaque in the RCA mid segment.

\section{Survival analysis}

In figure 3, Kaplan Meier curves were calculated to A) assess the difference between men and women with obstructive CAD and non-obstructive/no $\mathrm{CAD}$, respectively, and $\mathrm{B}$ ) to assess the difference between men and women with any $\mathrm{CAD}$ and no CAD, respectively. The presence of obstructive CAD as well as any CAD clearly resulted in a worse outcome in both men and women. Nonetheless, especially within patients without CAD, women had a visually worse prognosis as compared with men (figure 1-B). In table 4, the univariable prognostic value to predict for the combined endpoint is described. Next to several important clinical parameters, a higher calcium score, a higher number of plaques and presence of any or obstructive CAD were important predictors for the combined endpoint. Further, presence of plaques with any non calcified or partially calcified content predicted for the occurrence of events.

The prognostic value was further investigated using Cox regression models. The hazard ratio (HR) of obstructive CAD to predict for the combined endpoint in men was 37.2 (95\% confidence interval; 8.9-154.3; $\mathrm{p}<0.001)$. In women, the HR was 18.4 ( $95 \%$ confidence interval; 6.2-54.4; $\mathrm{p}<0.001)$. When corrected for age, smoking, diabetes, family history, systolic blood pressure, body mass index, total cholesterol and typical chest pain the HR of obstructive CAD to predict for events in women was 18.4 (95\% confidence interval; 6.2$54.4, \mathrm{p}<0.001)$. Also, the presence of typical chest pain was an independent predictor in women; HR 2.8 (95\% confidence interval; $1.1-6.9, \mathrm{p}=0.03)$. In men, only the presence of obstructive $\mathrm{CAD}$ was an independent predictor; $\mathrm{HR}$ 36.9 (95\% confidence interval 8.9-153.3, $\mathrm{p}<0.001)$. In the whole population, 
only obstructive CAD was an independent predictor for events; HR 22.9 (95\% confidence interval 7.1-73.7). Gender did not predict for outcome; HR 1.1 (95\% confidence interval $0.6-1.9, \mathrm{p}=0.71)$.

Table 4. Univariable predictors for coronary events during follow-up

\begin{tabular}{lccc} 
& HR & 95\% confidence interval & P-value \\
\hline Age (years) & 1.05 & $1.02-1.07$ & 0.001 \\
Male gender & 1.54 & $0.91-2.60$ & 0.11 \\
Smoking & 1.83 & $1.07-3.14$ & 0.03 \\
Diabetes & 0.96 & $0.39-2.40$ & 0.93 \\
Positive family history & 1.00 & $0.59-1.69$ & 1.00 \\
Typical chest pain & 1.34 & $0.66-2.72$ & 0.42 \\
BMI (kg/m ${ }^{2}$ ) & 0.95 & $0.89-1.01$ & 0.10 \\
Systolic blood pressure (mmHg) & 1.01 & $1.00-1.02$ & 0.07 \\
Total cholesterol (mmol/L) & 1.10 & $0.90-1.35$ & 0.36 \\
HDL cholesterol (mmol/L) & 0.67 & $0.35-1.28$ & 0.23 \\
LDL cholesterol (mmol/L) & 1.06 & $0.84-1.33$ & 0.64 \\
Triglycerides (mmol/L) & 1.03 & $0.86-1.23$ & 0.74 \\
Statin use & 2.00 & $1.21-3.32$ & 0.007 \\
Antihypertensive agent use & 1.91 & $1.10-3.32$ & 0.02 \\
Framingham risk score & 1.03 & $1.02-1.05$ & $<0.001$ \\
Calcium score & 1.001 & $1.001-1.002$ & $<0.001$ \\
Any CAD & 11.25 & $3.52-35.93$ & $<0.001$ \\
Obstructive CAD ( $\geq 50 \%$ stenosis) & 25.09 & $10.79-58.33$ & $<0.001$ \\
Segment stenosis score & 1.17 & $1.13-1.20$ & $<0.001$ \\
Number of plaques & 1.31 & $1.23-1.40$ & $<0.001$ \\
Number of non calcified plaques & 1.72 & $1.44-2.05$ & $<0.001$ \\
Number of mixed plaques & 1.55 & $1.38-1.73$ & $<0.001$ \\
Number of calcified plaques & 1.25 & $1.15-1.36$ & $<0.001$ \\
Any non-calcified or mixed plaque & 10.43 & $4.49-24.25$ & \\
\hline
\end{tabular}

Univariable hazard ratios to predict for occurrence of the combined endpoint. BMI, body mass index; CAD, coronary artery disease; HDL, high-density lipoprotein; LDL, low-density lipoprotein. 
Figure 3. Kaplan Meier analysis for men and women with different degrees of CAD
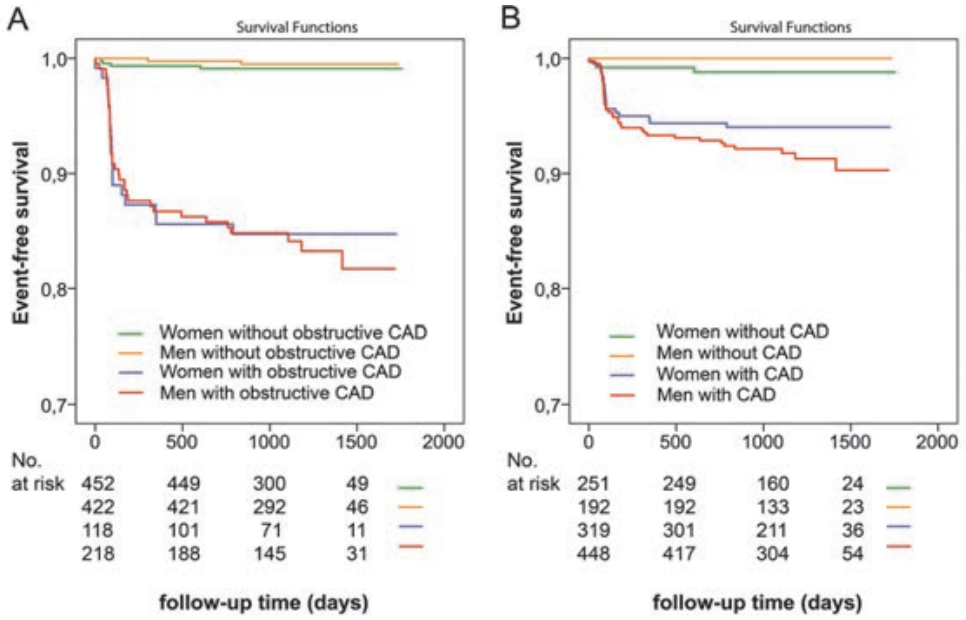

Kaplan Meier curves were calculated to A) assess the difference between men and women with obstructive $\mathrm{CAD}$ and non-obstructive/no CAD, respectively, and B) to assess the difference between men and women with any CAD and no CAD, respectively.

\section{DISCUSSION}

In the current study we investigated the prognostic value of CCTA in men and women. The presence of obstructive CAD on CCTA was a strong independent predictor for the occurrence of a coronary event, in both men and women, although the hazard ratio in men was approximately twice as high. Importantly, in women with previously non-obstructive or even normal CCTA findings, events were not uncommon. This in contrast to men, who showed no events when the previous CCTA was normal.

Overall, women referred for CCTA had a more disadvantageous risk profile. It seems that more risk factors were required for women to be referred for CCTA as compared with men. This is in line with several reports, of which some conclude that women are relatively under diagnosed. ${ }^{22,}{ }^{23}$ Nonetheless, women showed a more favorable CT-angiographic coronary status as compared with men. Calcium score, coronary stenosis and number of plaques were all significantly lower in women. Despite this, the event rate in men and woman was similar. Therefore, our results are in concurrence with the observation that women presenting with coronary events have less extensive CAD than men. ${ }^{10}$, 12 
The presence of obstructive CAD was the strongest predictor for a coronary event. Remarkably, also the use of statins and antihypertensive agents were predictors for the occurrence of an event. This is likely explained by the fact that these medications are prescribed to high risk patients (the significant association disappeared when corrected for FRS). Another important predictor was the presence of any non-calcified or mixed plaque. In addition, no events were found in patients with solely calcified plaques. The fact that women had a significantly higher proportion of non-calcified plaques as compared with men might explain their relatively high event rate, while having little overall atherosclerotic burden.

Unfortunately, large studies investigating gender difference in the prognostic value of CCTA are limited. The largest study investigating the prognostic value of CCTA in men and women is the study by Min et al. reporting on the CONFIRM trial. ${ }^{24}$ They concluded the prognostic value of CCTA for all-cause mortality is equal in both men and women. Although all-cause mortality of course is a very strong endpoint, non-cardiac deaths are not inconceivable in an intermediate risk population undergoing CCTA. In our population for instance, most deaths $(73 \%)$ were of non-cardiac origin. Shaw et al. reported that the extent of non-obstructive CAD is predictive for coronary events in women only. 25 This would also implicate that women with non-obstructive CAD developed coronary events more often. Another study stratified patients undergoing CCTA according to age and gender. ${ }^{26}$ Although the overall prognostic value was similar, CCTA did not predict for events in female patients under 60 years. These findings are in line with our results, as we report a trend towards more events in women with non-obstructive CAD as compared with men with nonobstructive CAD. Above all, within the event group, the proportion of patients with a normal CCTA was significantly higher in women as compared with men. Thus, in contrast to men, some female patients with a normal CCTA still developed coronary events. Although most other studies reported that a normal CCTA is accompanied by an excellent prognosis, events were not exceptional. The annualized event rate of a normal CCTA was $0.22 \%$ in our study, which is comparable to other literature. ${ }^{1,24,27,28}$ Remarkably, these events were all cases of ACS in female patients who initially presented with typical chest pain. This is in line with previous research reporting that women with typical chest pain still developed adverse events, despite 'normal' coronaries on conventional angiography. ${ }^{29}$ It has often been stated that coronary events despite a normal conventional angiogram could be explained by atherosclerosis in the vessel wall (as 
detected by IVUS). ${ }^{30}$ We hereby demonstrate that, although CCTA is able to visualize the vessel wall, also a normal CCTA did not preclude subsequent events. Therefore, the occurrence of plaque erosion in these three women may be unlikely. Possible other mechanisms for these events could have been endothelial dysfunction, coronary spasm or an obscure source of embolization. Such pathophysiological mechanisms leading to coronary events have been especially described in women. ${ }^{10,12-14}$

It has been reported that the presence of spasm or endothelial dysfunction is accompanied by a higher likelihood of future ACS. ${ }^{31,32}$ Our observation that women with typical chest pain but a normal CCTA had a relatively high ACS rate could make further diagnostic testing and risk stratification in these patients desirable. One approach could be ambulatory ECG-monitoring to assess markers of transient ischemia such as ST-segment changes and arrhythmias. To diagnose coronary endothelial dysfunction, an acetylcholine provocation tests could be employed. ${ }^{33}$ However, due to its non-invasive nature and close correlation to coronary endothelial function, a flow mediated vasodilatation test in the brachial artery might be a preferable test. ${ }^{34,} 35$ Identifying women with a high likelihood of endothelial dysfunction could help to determine their risk for coronary events. ${ }^{36,37}$ It is unfortunate to observe retrospectively that in our population, vasoprotective medication (including statins) was stopped based on a normal CCTA in all three patients who developed ACS. Aggressive antiatherogenic therapy such as statin treatment could aid endothelial function. ${ }^{38}$ Also angiotensin-converting enzyme inhibitors and of course lifestyle changes (e.g. stopping cigarette smoking, exercise) could be beneficial. ${ }^{39}$ A pragmatic approach could also be to test the anti-anginal effect of medication, such as calcium channel blockers, beta-blockers or nitrates.

In summary, we report an overall prognostic value of obstructive CAD on CCTA which is in concurrence with other studies. However, the prognosis of women with typical chest pain symptoms despite a normal CCTA was not as good as expected. We hypothesize that endothelial dysfunction might play a pivotal role in both symptoms, as well as coronary events in this patient group. It is therefore possible that endothelial testing could aid further risk stratification.

\section{Study limitations}

This is a single center, observational study, with a limited number of events. Treating physicians used CCTA in their clinical work-up, and subsequent 
treatment could have influenced event rates. The pathological mechanisms causing the events were not elucidated in our study. For instance, intravascular ultrasound at the time of the event could have provided additional information. Also, additional diagnostic testing investigating endothelial function could have provided important information. At the time however, we did not perform such measurements. A prospective study incorporating endothelial function tests is therefore desirable. The use of late revascularizations as an endpoint is often used. However, CCTA results could still have influenced clinical decisions on revascularization. By excluding procedures $<60$ days of CCTA, we have limited this potential bias.

\section{Conclusions}

The presence of obstructive CAD on CCTA is a strong predictor for the occurrence of a coronary event, in both men and women. However, in women with previously non-obstructive or even normal CCTA findings, events were not uncommon and occurred significantly more often as compared with men.

\section{References}

1. Hadamitzky M, Freissmuth B, Meyer T, et al. Prognostic value of coronary computed tomographic angiography for prediction of cardiac events in patients with suspected coronary artery disease. JACC Cardiovasc Imaging 2009;2:404-11.

2. Carrigan TP, Nair D, Schoenhagen P, et al. Prognostic utility of 64-slice computed tomography in patients with suspected but no documented coronary artery disease. Eur Heart J 2009;30:362-71.

3. Hadamitzky M, Distler R, Meyer T, et al. Prognostic value of coronary computed tomographic angiography in comparison with calcium scoring and clinical risk scores. Circ Cardiovasc Imaging 2011;4:16-23.

4. Versteylen MO, Joosen IA, Winkens MH, et al. Combined use of exercise electrocardiography, coronary calcium score and cardiac CT angiography for the prediction of major cardiovascular .events in patients presenting with stable chest pain. Int J Cardiol 2013; 167: 121-125.

5. Rubinshtein R, Halon DA, Gaspar T, Peled N, Lewis BS. Cardiac computed tomographic angiography for risk stratification and prediction of late cardiovascular outcome events in patients with a chest pain syndrome. Int J Cardiol 2009;137:108-15. 
6. Min JK, Shaw LJ, Devereux RB, et al. Prognostic value of multidetector coronary computed tomographic angiography for prediction of all-cause mortality. J Am Coll Cardiol 2007;50:1161-70.

7. Chow BJ, Small G, Yam Y, et al. Incremental prognostic value of cardiac computed tomography in coronary artery disease using CONFIRM: COroNary computed tomography angiography evaluation for clinical outcomes: an InteRnational Multicenter registry. Circ Cardiovasc Imaging 2011;4:463-72.

8. Lin FY, Shaw LJ, Dunning AM, et al. Mortality risk in symptomatic patients with nonobstructive coronary artery disease: a prospective 2-center study of 2,583 patients undergoing 64-detector row coronary computed tomographic angiography. J Am Coll Cardiol 2011;58:510-9.

9. Ambrose JA, Tannenbaum MA, Alexopoulos D, et al. Angiographic progression of coronary artery disease and the development of myocardial infarction. J Am Coll Cardiol 1988;12:56-62.

10. Lansky AJ, Ng VG, Maehara A, et al. Gender and the extent of coronary atherosclerosis, plaque composition, and clinical outcomes in acute coronary syndromes. JACC Cardiovasc Imaging 2012;5:S62-72.

11. Virmani R, Burke AP, Farb A, Kolodgie FD. Pathology of the vulnerable plaque. J Am Coll Cardiol 2006;47:C13-8.

12. Farb A, Burke AP, Tang AL, et al. Coronary plaque erosion without rupture into a lipid core. A frequent cause of coronary thrombosis in sudden coronary death. Circulation 1996;93:1354-63.

13. Burke AP, Farb A, Malcom GT, Liang Y, Smialek J, Virmani R. Effect of risk factors on the mechanism of acute thrombosis and sudden coronary death in women. Circulation 1998;97:2110-6.

14. Kramer MC, Rittersma SZ, de Winter RJ, et al. Relationship of thrombus healing to underlying plaque morphology in sudden coronary death. J Am Coll Cardiol 2010;55:122-32.

15. D'Agostino RB, Sr., Vasan RS, Pencina MJ, et al. General cardiovascular risk profile for use in primary care: the Framingham Heart Study. Circulation 2008;117:743-53.

16. Agatston AS, Janowitz WR, Hildner FJ, Zusmer NR, Viamonte M, Jr., Detrano R. Quantification of coronary artery calcium using ultrafast computed tomography. J Am Coll Cardiol 1990;15:827-32.

17. Versteylen MO, Joosen IA, Shaw LJ, Narula J, Hofstra L. Comparison of Framingham, PROCAM, SCORE, and Diamond Forrester to predict coronary atherosclerosis and cardiovascular events. J Nucl Cardiol 2011;18:904-11.

18. Shrimpton P. Assessment of patient dose in CT: appendix C-European guidelines for multislice computed tomography. European Commission project MSCT: CT safety \& efficacy—a broad perspective. http://www.msct.eu/PDF_FILES/Appendix\%20paediatric\%20CT\%20Dosimetry.pdf Published 2004. Accessed November 20, 2008. 
19. Raff GL, Abidov A, Achenbach S, et al. SCCT guidelines for the interpretation and reporting of coronary computed tomographic angiography. J Cardiovasc Comput Tomogr 2009;3:122-36.

20. Thygesen K, Alpert JS, White HD. Universal definition of myocardial infarction. J Am Coll Cardiol 2007;50:2173-95.

21. Braunwald E, Antman EM, Beasley JW, et al. ACC/AHA guidelines for the management of patients with unstable angina and non-ST-segment elevation myocardial infarction. A report of the American College of Cardiology/American Heart Association Task Force on Practice Guidelines (Committee on the Management of Patients With Unstable Angina). J Am Coll Cardiol 2000;36:970-1062.

22. Steg PG, Greenlaw N, Tardif JC, et al. Women and men with stable coronary artery disease have similar clinical outcomes: insights from the international prospective CLARIFY registry. Eur Heart J 2012;33:2831-40

23. Shaw LJ, Miller DD, Romeis JC, Kargl D, Younis LT, Chaitman BR. Gender differences in the noninvasive evaluation and management of patients with suspected coronary artery disease. Ann Intern Med 1994;120:559-66.

24. Min JK, Dunning A, Lin FY, et al. Age- and sex-related differences in all-cause mortality risk based on coronary computed tomography angiography findings results from the International Multicenter CONFIRM (Coronary CT Angiography Evaluation for Clinical Outcomes: An International Multicenter Registry) of 23,854 patients without known coronary artery disease. J Am Coll Cardiol 2011;58:849-60.

25. Shaw LJ, Min JK, Narula J, et al. Sex differences in mortality associated with computed tomographic angiographic measurements of obstructive and nonobstructive coronary artery disease: an exploratory analysis. Circ Cardiovasc Imaging 2010;3:473-81.

26. Yiu KH, de Graaf FR, Schuijf JD, et al. Age- and gender-specific differences in the prognostic value of CT coronary angiography. Heart 2012;98:232-7.

27. Hulten EA, Carbonaro S, Petrillo SP, Mitchell JD, Villines TC. Prognostic Value of Cardiac Computed Tomography Angiography A Systematic Review and Meta-Analysis. J Am Coll Cardiol 2011;57:1237-47.

28. Russo V, Zavalloni A, Bacchi Reggiani ML, et al. Incremental prognostic value of coronary CT angiography in patients with suspected coronary artery disease. Circ Cardiovasc Imaging 2010;3:351-9.

29. Diver DJ, Bier JD, Ferreira PE, et al. Clinical and arteriographic characterization of patients with unstable angina without critical coronary arterial narrowing (from the TIMI-IIIA Trial). Am J Cardiol 1994;74:531-7.

30. Erbel R, Ge J, Bockisch A, et al. Value of intracoronary ultrasound and Doppler in the differentiation of angiographically normal coronary arteries: a prospective study in patients with angina pectoris. Eur Heart J 1996;17:880-9.

31. Bugiardini R, Bairey Merz CN. Angina with "normal" coronary arteries: a changing philosophy. JAMA 2005;293:477-84. 
32. Quyyumi AA. Prognostic value of endothelial function. Am J Cardiol 2003;91:19H-24H.

33. Bugiardini R, Manfrini O, Pizzi C, Fontana F, Morgagni G. Endothelial function predicts future development of coronary artery disease: a study of women with chest pain and normal coronary angiograms. Circulation 2004;109:2518-23.

34. Celermajer DS, Sorensen KE, Gooch VM, et al. Non-invasive detection of endothelial dysfunction in children and adults at risk of atherosclerosis. Lancet 1992;340:1111-5.

35. Anderson TJ, Uehata A, Gerhard MD, et al. Close relation of endothelial function in the human coronary and peripheral circulations. J Am Coll Cardiol 1995;26:1235-41.

36. Schachinger V, Britten MB, Zeiher AM. Prognostic impact of coronary vasodilator dysfunction on adverse long-term outcome of coronary heart disease. Circulation 2000;101:1899-906.

37. Halcox JP, Schenke WH, Zalos G, et al. Prognostic value of coronary vascular endothelial dysfunction. Circulation 2002;106:653-8.

38. Kayikcioglu M, Payzin S, Yavuzgil O, Kultursay H, Can LH, Soydan I. Benefits of statin treatment in cardiac syndrome-X. Eur Heart J 2003;24:1999-2005.

39. Pizzi C, Manfrini O, Fontana F, Bugiardini R. Angiotensin-converting enzyme inhibitors and 3-hydroxy-3-methylglutaryl coenzyme A reductase in cardiac Syndrome X: role of superoxide dismutase activity. Circulation 2004;109:53-8. 


\section{Chapter}

\section{Additive Value of Semi-automated quantification of Coronary Artery Disease using Cardiac CT Angiography to predict for Future Acute Coronary Syndrome}

Mathijs O. Versteylen, Bas L. Kietselaer, Pieter C. Dagnelie, Ivo A. Joosen, Admir Dedic, Rolf H. Raaijmakers, Joachim E. Wildberger, Koen Nieman, Harry J. Crijns, Wiro J. Niessen, Mat J. Daemen, Leonard Hofstra

$J$ Am Coll of Cardiol. 2013; 61(22): 2296-2305. 


\section{ABSTRACT}

Objectives To investigate whether the use of a semi-automated plaque quantification algorithm (reporting volumetric and geometric plaque properties) provides additional prognostic value for the development of acute coronary syndrome (ACS) as compared with conventional reading from cardiac computed tomographic angiography (CCTA).

Background CCTA enables the visualization of coronary plaque characteristics, of which some have been shown to predict ACS.

Methods A total of 1,650 patients underwent 64-slice CCTA and were followedup for ACS for a mean $26 \pm 10$ months. In 25 patients who developed ACS and 101 random controls (selected from 993 patients with coronary artery disease, but without coronary event), coronary artery disease was evaluated using conventional reading (calcium score, luminal stenosis, morphology), and then independently quantified using semi-automated software (plaque volume, burden [plaque area/vessel area $* 100 \%]$, area, non-calcified percentage, attenuation, remodeling). Clinical risk profile was calculated with Framingham risk score (FRS).

Results There were no significant differences in conventional reading parameters between controls and patients who developed ACS. Semi-automated plaque quantification showed that compared to controls, ACS patients had higher total plaque volume (median 94 vs. $29 \mathrm{~mm}^{3}$ ) and total non-calcified volume ( 28 vs. $4 \mathrm{~mm}^{3}, \mathrm{p} \leq 0.001$ for both). In addition, per plaque maximal volume (median 56 vs. $24 \mathrm{~mm}^{3}$ ), non-calcified percentage (62 vs. 26\%) and plaque burden (57 vs. $36 \%$ ) in ACS patients were significantly higher ( $\mathrm{p}<0.01$ for all). An receiver operating characteristic (ROC)-model predicting for ACS incorporating FRS and conventional CCTA-reading had an area under the curve (AUC) of 0.64 , a second model also incorporating semi-automated plaque quantification had an AUC of 0.79, $\mathrm{p}<0.05$.

Conclusion The semi-automated plaque quantification algorithm identified several parameters predictive for ACS and provided incremental prognostic value over clinical risk profile and conventional CT-reading. The application of this tool may improve risk stratification in patients undergoing CCTA. 


\section{INTRODUCTION}

Currently, > 1 million Americans per year suffer from acute coronary syndrome (ACS). ${ }^{1}$ Although risk stratification in combination with preventive measures taken on a population level has resulted in a decline in the number of events in Western countries, the assessment of cardiovascular risk in a single individual is still a highly challenging task.

One avenue towards a more tailored approach may be the effective use of imaging diagnostics. Following the introduction of coronary calcium scoring, coronary computed tomographic angiography (CCTA) has been widely implemented in clinical practice during the last decade. ${ }^{2,3}$ CCTA has shown its ability to effectively rule out coronary artery disease (CAD). Further, the predictive value of CCTA seems promising, in particular using maximal luminal stenosis severity as a prognostic parameter. ${ }^{4,5}$ However, in addition to luminal stenosis, CCTA is capable to identify several morphologic and geometric characteristics of atherosclerotic plaques. ${ }^{6}$ Positive plaque remodeling and the presence of low-attenuation plaque core have been identified as risk factors for the occurrence of ACS. 7,8 Nevertheless, these characteristics are not regularly reported and reliable measurement is problematic due to lack of standardization. In addition, a semi-automated approach is required for plaque volume measurements. Consequently, the potential of the available imaging information present in CCTA datasets is currently not fully exploited.

Several methods to quantify coronary plaque morphology have been successfully validated against intravascular ultrasound. ${ }^{910}$ However, clinical data on incremental value of plaque quantification over conventional reading of coronary artery disease using CCTA (i.e. calcium score, luminal stenosis severity, etc.) are lacking. We hypothesize that more extensive plaque quantification could maximize the prognostic value of CCTA in terms of the assessment of cardiovascular risk in a single individual. The goal of this study was to investigate the additional predictive value of the use of a semi-automated quantification algorithm over conventional CCTA reading (consisting of calcium score, luminal stenosis, extent of CAD and morphology assessment). 


\section{METHODS}

\section{Study population}

We retrospectively analyzed a cohort of 2,054 stable chest pain patients who were referred for CCTA, from the Maastricht University Medical Center $(n=1,599)$ and the Erasmus MC, University Medical Center Rotterdam $(\mathrm{n}=455)$. All CCTAs were performed between September 2006 until June 2010. Included were patients who underwent both a calcium score scan as well as CTangiography. MUMC protocol precluded CCTA when calcium score was $>1000$. CCTA was also not performed in patients with unstable angina, hemodynamic instability, pregnancy, renal insufficiency and severe iodine allergy. Excluded from the present analysis were 94 patients who had a history of CAD, 162 patients who had an inconclusive CCTA and in 148 patients clinical data were missing.

The remaining 1,650 patients were followed up for a mean $26 \pm 10$ months. Electronic patient records were checked for occurrence of ACS, including myocardial infarction and unstable angina. ACS was defined as typical angina pectoris and troponin T elevation $(>0.01 \mu \mathrm{g} / \mathrm{L})$ and ST-segment elevation/depression of $\geq 1 \mathrm{~mm}$, or at least two of these symptoms together with invasive angiographic confirmation of a culprit lesion. ${ }^{11}$ In addition, occurrence of all-cause mortality and revascularization procedures were registered, to guarantee the control population was free of cardiovascular events. All events were confirmed with the national death registry, hospital records, or correspondence with hospitals and treating physicians. An independent cardiologist blinded for CCTA results reviewed the events.

During the follow-up period, 29 patients developed ACS. Four ACS cases were excluded because no definite culprit lesion could be identified during invasive coronary angiography, or the culprit lesion could not be correlated to CCTA findings. In two of these patients a culprit lesion in the RCA mid segment was detected by conventional angiography. Unfortunately, the corresponding segment on CCTA was blurred by motion artifacts and no plaque could be identified. Although this exclusion could improve the performance of CCTA in general, we felt this was reasonable to do since our analysis is based on comparing conventional with semi-automatically quantified CCTA. In the third patient, CCTA revealed no CAD, which was confirmed by conventional angiography. In the fourth the culprit vessel during conventional angiography was a first diagonal branch, nevertheless, no plaque could be objectified on the 
CCTA images. An infarct due to thromboembolism was suspected. After identification of the ACS patients, the control population was selected. From the 993 remaining patients who revealed any CAD on their CCTA and suffered no cardiovascular events during follow-up, we randomly selected 101 patients. The study protocol conforms to the ethical guidelines of the 1975 Declaration of Helsinki. The Institutional Review Board and ethics committee approved the study and all patients signed informed consent.

In all patients, clinical risk factors were prospectively collected. The Framingham risk score (FRS) was calculated to reflect clinical risk for cardiovascular events. $^{12}$

\section{CCTA acquisition}

At Maastricht University Medical Center, CCTA was performed using a 64-slice multi detector computed tomography scanner (Brilliance 64; Philips Healthcare, Best, The Netherlands) with a $64 \times 0.625 \mathrm{~mm}$ slice collimation, a rotation time of $420 \mathrm{~ms}$, a tube voltage of 80 or $120 \mathrm{kV}$ and the tube current varied from 150 to $1000 \mathrm{mAs}$, depending on the patient's height and weight. Patients received a contrast bolus of $85-110 \mathrm{ml}$, injected at a rate of $5.0-7.2 \mathrm{ml} / \mathrm{s}$. In patients with heart rate $<65 \mathrm{bpm}$, a prospective ECG-triggered axial scan was performed, in patients with heart rate $>65 \mathrm{bpm}$, a retrospective ECG-triggered spiral acquisition protocol was used. Other scan details were previously described. ${ }^{13}$

At Erasmus Medical Center, CCTA was performed using a first generation dual-source CT scanner (Siemens Definition, Forchheim, Germany) with 2 x $32 \times 0.6 \mathrm{~mm}$ slice collimation, a rotation time of $330 \mathrm{~ms}$, a tube voltage of $120 \mathrm{kV}$ and the tube current varied from $380-412 \mathrm{~mA}$, depending on patient's height and weight. A contrast bolus of $70-100 \mathrm{~mL}$ was injected at $5.0-5.5 \mathrm{ml} / \mathrm{s}$. A spiral acquisition protocol was used, with ECG-triggered dose modulation in all patients with stable heart rates. Further characteristics were previously described in detail. ${ }^{14}$

In both centers, a native scan using $120 \mathrm{kV}$ and $3 \mathrm{~mm}$ slice thickness was performed to determine the calcium score. Mean total estimated radiation dose was calculated by multiplying the dose-length product by the conversion factor of $0.014 \mathrm{mSv} / \mathrm{mGy} / \mathrm{cm}$ for the thorax. ${ }^{15}$ 


\section{Conventional CCTA reading}

Calcium score was quantified by Agatston method. ${ }^{16} \mathrm{CT}$ angiograms were independently analyzed by a cardiologist and a radiologist both experienced in reading cardiac CT and blinded to clinical information, using the American Heart Association 16 coronary segments model. ${ }^{17}$ In case of disagreement, consensus was reached by discussion. The coronary artery tree was assessed using the source images on the provided software (Comprehensive Cardiac, Philips Healthcare). The degree of stenosis was visually defined and a lesion severity score was calculated as follows: no lesion (score 0 ), mild diameter stenosis $(<50 \%$, score 1$)$, moderate $(50-70 \%$, score 2$)$ and severe diameter stenosis $(>70 \%$, score 3$)$. This resulted in a lesion severity score ranging from $0-3$ for every individual patient. A segment stenosis score was the sum of the lesion severity in all 16 segments (range 0-48). ${ }^{18}$ Plaques were categorized as calcified (exclusively content with density $>130$ Hounsfield units), non-calcified (exclusively content with density $<130$ Hounsfield units), or partially calcified (characteristics of both calcified and non-calcified plaque). For each patient the number of non-calcified plaques and partially calcified plaques was counted to reflect the presence on non-calcified plaque content in the coronary artery tree (number of non-calcified segments-score, range 0-16).

\section{Semi-automated plaque quantification}

Quantification of CAD in the entire coronary artery tree was performed using a dedicated software algorithm (Comprehensive Cardiac Analysis (CCA), version 4.5.2.40007, Philips Healthcare), figure 1. The analysis consisted of the following automated steps: segmentation of the heart, coronary tree and centerline extraction, and vessel contouring. Subsequently, plaques were manually identified, followed by automatic segmentation, characterization and quantification. Manual corrections to centerline, vessel contours and lesion segmentation could be made by the observer, as described before. ${ }^{19}$ In case multiple reconstructed phases were available, the best phase to assess an entire coronary artery was selected. Individual segments that were not interpretable were not assessed and regarded as normal. A subgroup of 40 patients were analyzed twice by the same reviewer (blinded, after a 4 week interval), as well as a different reviewer, to asses intra- and inter-observer variability. Plaque characteristics reported by this algorithm have been reported to be reproducible, ${ }^{20,21}$ and stenosis severity correlated well with conventional angiography. ${ }^{19}$ 


\section{Plaque quantification parameters}

The semi-automated software reported the following cross-sectional plaque characteristics per plaque analyzed: maximal cross-sectional plaque area, maximal plaque burden (plaque area/vessel area $* 100 \%$ ); remodeling index, as well as volumetric measurements of the plaque. Also, mean attenuation of the entire plaque in Hounsfield units (HU) and composition (percentage calcified and non-calcified morphology) were reported per plaque.

In addition to per plaque parameters that were reported by the software algorithm, several parameters were calculated to enable a per patient analysis. We calculated total plaque volume and total non-calcified plaque volume per patient. Because the total plaque characteristics do not per se appreciate the characteristics of individual plaques, we also investigated maximum plaque values per patient (minimum for plaque attenuation). We calculated the highest plaque volume, highest plaque burden, highest plaque area, highest remodeling index, lowest plaque attenuation and highest non-calcified percentage on a per patient basis.

\section{Culprit analysis}

In addition, within patients developing ACS, we compared semi-automatically quantified plaque characteristics between culprit and non-culprit lesions. Culprit lesions were identified using conventional angiography at the time of event, and the corresponding segment on CCTA was selected. 
Figure 1. Conventional reading versus semiautomated plaque quantification

Conventional reading
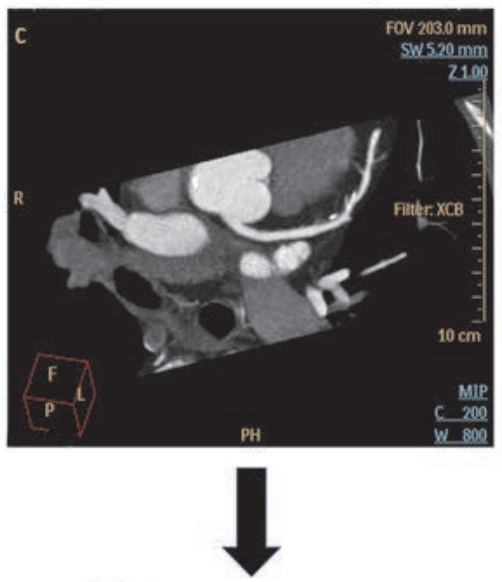

Calcium score

$\geq 50 \%$ stenosis

Segment stenosis score

Non-calcified plaque content
Semi-automated algorithm

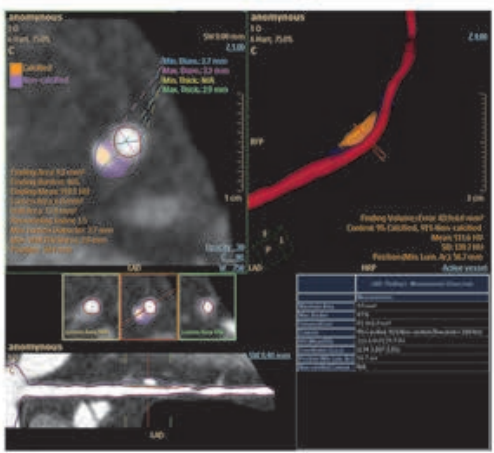

Plaque volume

Plaque burden

Plaque area

Plaque non-calcified percentage

Plaque attenuation

Plaque remodeling

Plaque analysis and used plaque parameters by conventional CT reading and by using the semiautomated quantification algorithm. In this example the mid segment of the left anterior descending coronary artery showed a partially calcified plaque.

\section{Statistics}

Data were analyzed using SPSS 19.0 (SPSS Inc., Chicago, IL, USA). Continuous baseline characteristics in table 1 were reported as means and standard deviations (SD), since all were normally distributed. Consequently, differences between groups were assessed using ANOVA. Normality of data distribution was evaluated using the Kolmogorov-Smirnov test. Since part of the continuous variables in tables 2 and 3 were not normally distributed, all values are uniformly reported as median interquartile range (IQR) for clarity. Consequently, here differences were assessed using Mann-Whitney test. Proportions (\%) were used for categorical values. Fisher's exact test was used for categorical variables. Inter- and intra-observer variability was tested by calculating interclass correlation coefficients (ICC), using a two-way mixed model. For all continuous parameters derived from CCTA analysis, receiver-operating-characteristics (ROC) curves were plotted and cut-off values determined to derive the maximum sum of sensitivity and specificity. Using these cut-off values, accuracy was calculated as follows: (number true positives + true negatives) / (true positives + false 
positives + true negatives + false negatives). Separate sum scores for the conventional CT parameters and for semi-automatically quantified CT parameters were calculated. The net reclassification index (NRI) was calculated to evaluate reclassification improvement of the quantification sum score as compared with the conventional sum score. ROC curves were plotted for four incremental models; FRS, FRS + calcium score, FRS + sum score conventional CT reading and FRS + sum score conventional CT reading + sum score semi-automated CT quantification. Area under the curve (AUC) for prediction of ACS by the four models was produced to evaluate the incremental value of the combined approach and difference between AUCs was tested for significance. ${ }^{22}$ All pvalues were 2 -sided, and a p-value below 0.05 was considered statistically significant.

\section{RESULTS}

\section{Study population}

Mean age of the total population was $63 \pm 10$ years, $75(60 \%)$ were male. Mean FRS was $26 \pm 19 \%$, and 105 patients (83\%) presented with complaints of atypical chest pain, $21(17 \%)$ with typical chest pain. A spiral protocol was used in $54(43 \%)$, prospective protocol in $72(57 \%)$ patients. Mean radiation dose was $5.7 \pm 3.6 \mathrm{mSv}$. Baseline characteristics for patients developing ACS and the control group are described in table 1 . In patients that subsequently suffered from ACS, the incidence of diabetes mellitus was significantly higher. There was a trend towards a higher incidence of smoking and a higher FRS in this group. In the ACS group, $4(16 \%)$ patients had at least one non-interpretable segment, which was $24(24 \%)$ for the control group, $\mathrm{p}=0.40$. The median number of non-interpretable segments per patient was $0(0-0)$ for both groups, $\mathrm{p}=0.51$. After CCTA, medical treatment did not differ between patients who later developed ACS and controls, aspirin: $16(64 \%)$ vs. $50(50 \%)$ p $=0.82$, antihypertensive agent: $16(64 \%)$ vs. $68(67 \%) \mathrm{p}=0.81$ and statin: $16(64 \%)$ vs. $74(73 \%)$ $\mathrm{p}=0.46$, respectively. 
Table 1. Baseline characteristics of the study population, for patients with and without ACS

\begin{tabular}{lccc} 
& $\begin{array}{c}\text { Patients with ACS } \\
(\mathrm{n}=25)\end{array}$ & $\begin{array}{c}\text { Patients without ACS } \\
(\mathrm{n}=101)\end{array}$ & P-value \\
\hline Age (years) & $60 \pm 11$ & $60 \pm 10$ & 0.78 \\
Male gender & $14(56 \%)$ & $61(60 \%)$ & 0.82 \\
Smoking & $9(36 \%)$ & $18(18 \%)$ & 0.06 \\
Diabetes & $6(24 \%)$ & $8(8 \%)$ & 0.03 \\
Positive family history & $10(40 \%)$ & $50(50 \%)$ & 0.50 \\
BMI $\left(\mathrm{kg} / \mathrm{m}^{2}\right)$ & $27 \pm 5$ & $28 \pm 5$ & 0.24 \\
Systolic blood pressure $(\mathrm{mmHg})$ & $152 \pm 23$ & $148 \pm 22$ & 0.52 \\
Total cholesterol $(\mathrm{mg} / \mathrm{dL})$ & $209 \pm 50$ & $211 \pm 55$ & 0.89 \\
Framingham risk score & $32 \pm 22$ & $25 \pm 17$ & 0.09 \\
\hline
\end{tabular}

Values are presented as numbers (\%), or means \pm standard deviation. Significant p-values are in bold. ACS=acute coronary syndrome, $\mathrm{BMI}=$ body mass index, $\mathrm{CCTA}=$ cardiac computed tomographic angiography.

\section{Inter- and intra-observer variability}

For total plaque volume the inter- and intra-observer correlation was 0.88 and 0.98 , respectively. The reproducibility of total non-calcified plaque volume was lower (inter-observer correlation 0.47 , and intra-observer correlation 0.86). A comprehensive overview of the reproducibility of all semi-automatically quantified parameters is provided in table S1.

\section{Predictive value CT parameters}

The predictive value of the different CT parameters was investigated by (nonadjusted) comparison of patients developing ACS with randomly selected control patients (table 2). Conventional CT-derived parameters, such as calcium score and lesion severity score, showed no significant differences between patients with and without ACS. By contrast, we found the following semiautomated quantitative parameters to be significantly higher in the ACS group: total plaque volume, total number of plaques and total non-calcified volume ( $\mathrm{p} \leq 0.02$ for all). Regarding maximal plaque values per patient, patients who developed an ACS during follow-up had a significantly higher maximal plaque volume, maximal plaque burden, maximal plaque area, maximal percentage non-calcified plaque, minimal plaque attenuation and maximal remodeling $(\mathrm{p} \leq 0.01$ for all, table 2$)$. 
Table 2. Difference between patients with and without ACS, for conventional CT reading and semiautomated quantitative parameters

\begin{tabular}{|c|c|c|c|}
\hline & $\begin{array}{l}\text { Patients with ACS } \\
\qquad(n=25)\end{array}$ & $\begin{array}{l}\text { Patients without } \\
\text { ACS }(n=101)\end{array}$ & P-value \\
\hline \multicolumn{4}{|l|}{ Conventional CT reading } \\
\hline Calcium score & $109(20-596)$ & $72(9-260)$ & 0.25 \\
\hline Lesion severity score (1-3) & $2(1-3)$ & $2(1-3)$ & 0.41 \\
\hline Segment stenosis score (1-48) & $6(3-11)$ & $4(2-8)$ & 0.14 \\
\hline Number non-calcified segments (1-16) & $2(1-3)$ & $1(1-3)$ & 0.20 \\
\hline \multicolumn{4}{|l|}{ Semi-automated quantification } \\
\hline \multicolumn{4}{|l|}{ Total values per patient } \\
\hline Total number of plaques & $4(2-6)$ & $2(1-4)$ & 0.02 \\
\hline Total plaque volume (mm3) & $94(62-175)$ & $29(8-113)$ & 0.001 \\
\hline Total non-calcified volume (mm3) & $28(16-59)$ & $4(0-20)$ & $<0.001$ \\
\hline \multicolumn{4}{|l|}{ Maximal plaque values } \\
\hline Plaque with highest volume (mm3) & $56(40-100)$ & $24(8-56)$ & $<0.001$ \\
\hline Plaque with highest plaque burden (\%) & $57(45-66)$ & $36(24-51)$ & $<0.001$ \\
\hline Plaque with highest area (mm2) & $9(6-9)$ & $5(2-8)$ & 0.006 \\
\hline Plaque with highest non-calcified \% & $62(25-100)$ & $26(0-70)$ & 0.006 \\
\hline Plaque with lowest attenuation (HU) & $206(118-292)$ & $282(179-377)$ & 0.005 \\
\hline Plaque with highest remodeling & $1.5(1.3-1.5)$ & $1.3(1.1-1.5)$ & 0.01 \\
\hline
\end{tabular}

Values are presented as median (IQR). Significant p-values are in bold. The quantitative parameters are presented as total values per patient (sum of all plaques) or mean values per patient (total divided by number of plaques). ACS = acute coronary syndrome, IQR = interquartile range, $\mathrm{HU}=$ Hounsfield units.

\section{Culprit analysis}

In addition, coronary plaques on CCTA corresponding with subsequent culprit lesions had different characteristics than plaques corresponding with nonculprit lesions, table 3. Plaque volumes, burden and area were significantly higher in culprit lesions, plaque attenuation was significantly lower. There was a trend towards higher remodeling index in culprit lesions. 
Table 3. Difference in semi-automatically quantified plaque characteristics between culprit lesions and non-culprit plaques within patients who developed ACS $(n=24)$

\begin{tabular}{lccc} 
& $\begin{array}{c}\text { Culprit plaques } \\
(\mathrm{n}=24)\end{array}$ & $\begin{array}{c}\text { Non-culprit plaques } \\
(\mathrm{n}=74)\end{array}$ & P-value \\
\hline Plaque total volume (mm3) & $25.4(7.3-69.6)$ & $9.5(1.9-43.8)$ & 0.04 \\
Plaque non-calcified volume (mm3) & $3.4(0-18.8)$ & $0(0-5.4)$ & 0.005 \\
Plaque burden (\%) & $39.0(24.5-56.0)$ & $29.0(12.0-39.3)$ & 0.02 \\
Plaque area (mm2) & $5.0(2.4-8.6)$ & $3.3(0.9-5.9)$ & 0.04 \\
Plaque attenuation (HU) & $287(195-543)$ & $468(323-557)$ & 0.04 \\
Plaque remodeling index & $1.3(1.2-1.5)$ & $1.2(1.0-1.4)$ & 0.06 \\
\hline
\end{tabular}

Variables are described as median (interquartile range). Significant p-values are in bold. NB: 24 culprit lesions were assessed, as one patient who presented with ACS did not undergo conventional angiography and as a consequence no culprit lesion could be objectified. ACS = acute coronary syndrome, $\mathrm{HU}=$ Hounsfield units.

\section{ROC analysis}

All continuous CT parameters were investigated using ROC analysis. Cut-off values achieving the highest sum of sensitivity and specificity were chosen (table 4). This was done for the parameters of conventional CT reading, as well as for semi-automated quantitative parameters. For the plaque quantitative parameters, the highest accuracy was achieved for total plaque volume and total noncalcified volume (for entire coronary tree, accuracy: 65 and 72, respectively), and for maximal plaque volume, maximal plaque burden and maximal noncalcified percentage (for the most severe plaque, accuracy: 62, 66, 63, respectively). ROC-curves for these parameters are shown in figure 2 . 
Table 4. ROC analysis of CT-derived parameters to predict for the occurrence of ACS

\begin{tabular}{lcccccc} 
& Cut-off & Sens & Spec & PPV & NPV & Accuracy \\
\hline Conventional CT reading & & & & & & \\
Calcium score & $>368$ & 38 & 16 & 38 & 84 & 75 \\
Lesion severity score (1-3). & $>2$ & 72 & 36 & 22 & 84 & 43 \\
Stenosis severity score (1-48) & $>5$ & 60 & 62 & 28 & 86 & 62 \\
Number non-calcified segments (1-16) & $>1$ & 64 & 51 & 24 & 83 & 61 \\
Semi-automated quantification & & & & & & \\
Total values per patient & & & & & & \\
$\quad$ Total number of plaques & $>2$ & 68 & 54 & 27 & 87 & 57 \\
$\quad$ Total plaque volume (mm3) & $>57.9$ & 80 & 61 & 34 & 93 & 65 \\
$\quad>13.8$ & 80 & 70 & 40 & 93 & 72 \\
Matal non-calcified volume (mm3) & & & & & & \\
$\quad$ Highest plaque volume (mm3) & $>28$ & 88 & 58 & 33 & 95 & 62 \\
$\quad$ Highest plaque burden (\%) & $>45$ & 80 & 62 & 35 & 93 & 66 \\
$\quad$ Highest plaque area (mm2) & $>5$ & 88 & 48 & 29 & 94 & 56 \\
$\quad$ Highest non-calcified percentage & $>44$ & 72 & 60 & 31 & 90 & 63 \\
$\quad$ Lowest plaque attenuation (HU) & $<316$ & 92 & 41 & 28 & 95 & 51 \\
$\quad$ Highest remodeling index & $>1.3$ & 92 & 46 & 30 & 96 & 55 \\
\hline
\end{tabular}

ROC analysis was performed for all continuous variables. Optimal cut-off values were selected to achieve the maximum sum of sensitivity and specificity. Accuracy was calculated by: (number true positives + true negatives) / (true positives + false positives + true negatives + false negatives).

Table S1. Reproducibility of semi-automated quantitative parameters

Intra-observer correlation $\quad \begin{gathered}\text { Inter-observer } \\ \text { correlation }\end{gathered}$

$\begin{array}{lll}\text { Total plaque values } & 0.97 & 0.74 \\ \text { Total number of plaques } & 0.98 & 0.88 \\ \text { Total plaque volume } & 0.86 & 0.47 \\ \text { Total non-calcified volume } & & \\ & & \\ \text { Highest plaque values } & 0.90 & 0.90 \\ \text { Plaque with highest volume } & 0.75 & 0.64 \\ \text { Plaque with highest plaque burden } & 0.51 & 0.80 \\ \text { Plaque with highest area } & 0.62 & 0.28 \\ \text { Plaque with highest non-calcified \% } & 0.63 & 0.26 \\ \text { Plaque with lowest attenuation } & 0.66 & 0.55 \\ \text { Plaque with highest remodeling } & \end{array}$

$\overline{\text { In a subgroup of } 40 \text { patients, intra- and inter-observer variability were tested using a ICC, two-way }}$ mixed model. 
Figure 2. ROC analysis of semi-automated quantitative plaque parameters predicting acute coronary syndrome
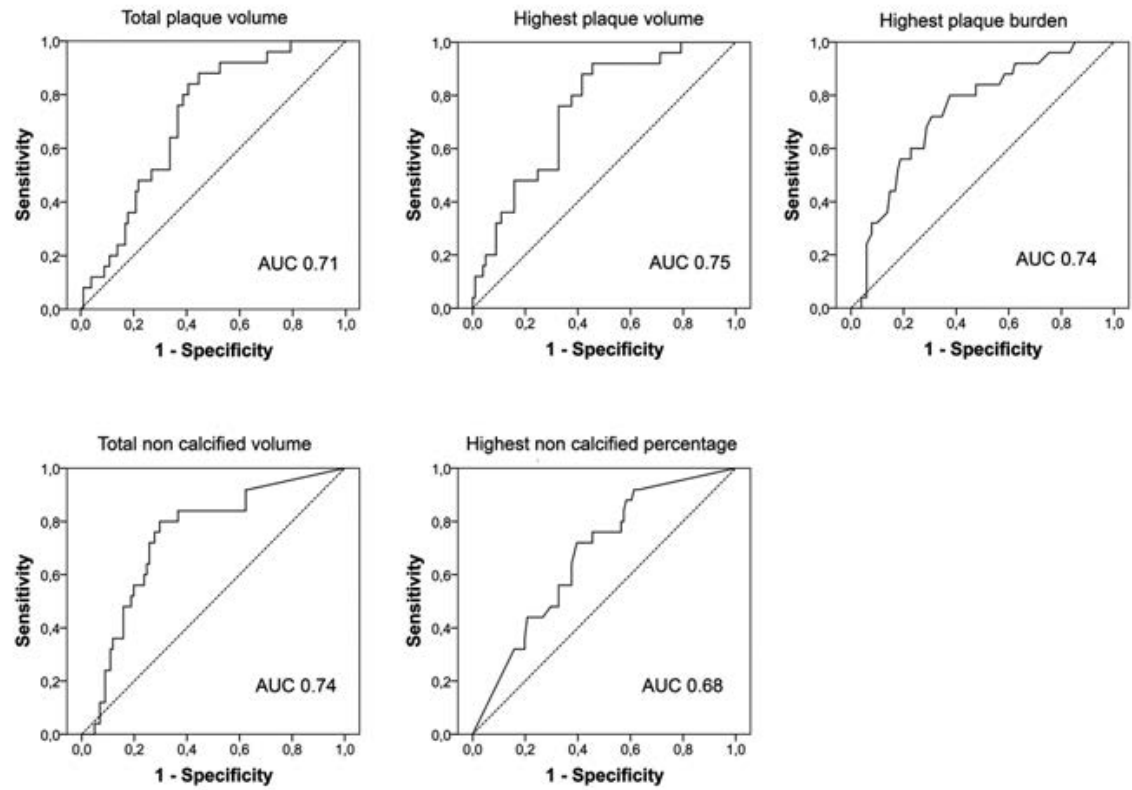

Receiver operating characteristic (ROC) curves for both total plaque volume and the highest plaque volume, total non-calcified volume and highest non-calcified percentage and highest plaque burden.

\section{Sum scores and reclassification}

Using the optimal cut-off values as reported in table 4, all characteristics were transformed into dichotomous variables. Using these variables, we calculated sum scores for the conventional CT parameters and for the 5 semi-automated quantitative CT parameters with the best accuracy. The sum score for the conventional parameters had a range of $0-4$, the sum score for the quantification parameters had a range of 0-5. Again, we achieved optimal cut-off values for both sum scores using ROC-analysis. The optimal cut-off level for the prediction of events for the conventional model was $>2$ (sensitivity $52 \%$, specificity $68 \%$, positive predictive value $29 \%$, negative predictive value $85 \%$ ), and for the quantification model the cut-off level was $>3$ (sensitivity $76 \%$, specificity $71 \%$, positive predictive value $40 \%$, negative predictive value $92 \%$ ). When the quantification model was compared with the conventional model, $24 \%$ of the event group was reclassified into the higher risk group, $3 \%$ in the non-event group was reclassified into the lower risk group. The NRI was $27 \%$. 


\section{Incremental value over clinical risk profiling}

To assess the added value of the different models over clinical risk profile, we compared four incremental models (figure 3). The first model consisted of the FRS to reflect the risk for the occurrence of ACS determined by clinical risk factors. The second model consisted of the FRS plus calcium score. The third model consisted of the FRS plus the sum score of the 4 conventional CT parameters. The fourth model consisted of FRS plus the sum score of the conventional CT parameters plus the sum score of the 5 semi-automated quantification algorithm's CT characteristics. The AUC for the FRS alone was 0.59 (95\% CI 0.45-0.73). When calcium score was added to FRS, the AUC increased to 0.67 (95\% CI $0.54-0.79, \mathrm{p}=0.17)$. The model incorporating FRS and conventional CT parameters showed an AUC of 0.64 (95\% CI 0.52-0.76, $\mathrm{p}=0.42)$. When the semi-automated quantification parameters were added, the AUC increased significantly to 0.79 ( $95 \%$ CI $0.69-0.90, \mathrm{p}=0.047)$.

Figure 3. Incremental value of semi-automated plaque quantification in predicting acute coronary syndrome

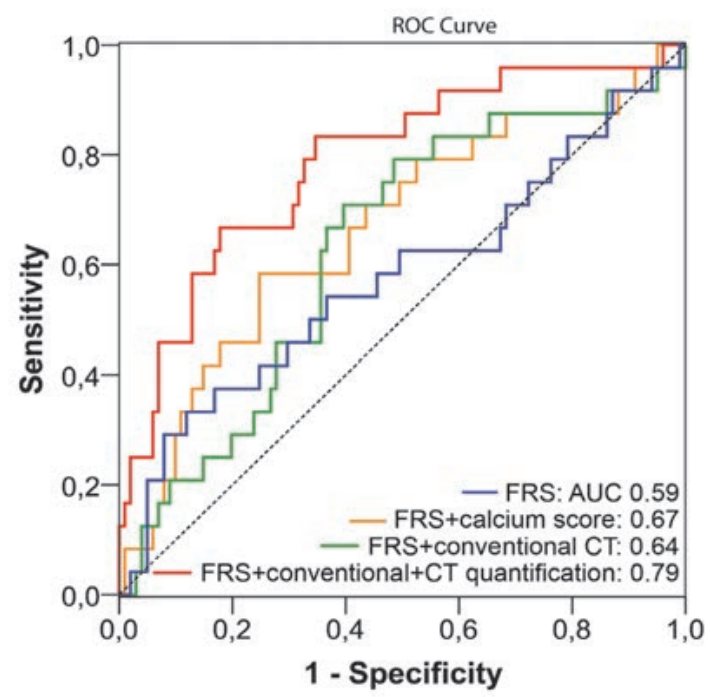

Receiver operating characteristic (ROC) curves of the four incremental models. Framingham risk score (FRS) alone, plus calcium score, plus conventional CCTA and FRS plus conventional CCTA and semi-automated plaque quantification. 


\section{DISCUSSION}

In this study, we investigated the use of a semi-automated plaque quantification algorithm to predict for ACS in stable chest pain patients undergoing CCTA. The main finding of this study was that semi-automatically quantified plaque characteristics provided additional prognostic value over both clinical risk profiling and conventional CT reading (including calcium score, lesion severity, segment stenosis score and number of segments with non-calcified plaques). These findings suggest that the clinical use of such a semi-automated plaque quantification algorithm might improve stratification for ACS risk in patients presenting with stable chest pain undergoing CCTA.

\section{Prognostic value of CCTA}

CCTA has become an attractive clinical tool for the non-invasive assessment of $\mathrm{CAD}$, with the goal to optimize risk stratification in the individual patient. Strong predictors for adverse outcomes reported in literature are the presence of a $\geq 50 \%$ stenosis, the number of coronary segments with CAD and the calcium score. $5,18,23$ Some studies have even shown these conventional CCTA parameters to have independent prognostic value over clinical risk profiling and calcium score. ${ }^{24-26}$ In our study, conventional CT showed no additive value over risk profiling. This can be explained by the difference in study design, especially the fact that we only selected patients with any CAD on their CCTA, which obviously decreased the ability of conventional CCTA to differentiate between high and low risk patients. Although promising, the above CCTA studies hardly provide information about plaque morphology and geometry, thereby not fully exploiting the potential of CCTA and the information that is present in the imaging datasets.

\section{Prognostic value of coronary plaque characteristics}

The use of CCTA to evaluate plaque characteristics is still subject to controversy. The accuracy of CCTA to identify plaque characteristics using intravascular ultrasound (IVUS) as the gold standard has been reported by several groups. $6,9,27,28$ The comparative studies between CCTA and IVUS have shown that partially calcified and calcified lesions can be quite accurately detected by CCTA, whereas non-calcified plaque volume is often underestimated. ${ }^{28,29}$ In a recent study, several plaque characteristics were investigated in a population undergoing IVUS after their first ACS. ${ }^{30}$ After follow-up, high plaque burden 
and low minimal luminal area were among the strongest predictors of a subsequent cardiovascular event in this study, which is in line with our findings.

Furthermore, several vulnerable plaque characteristics have been described that are associated with the occurrence of ACS, especially presence of non-calcified and partially calcified plaques, ${ }^{31,32}$ outward remodeling ${ }^{33}$ and a low attenuation of plaques. ${ }^{7,34-36}$ Also, total plaque volume has been reported to be significantly higher in patients presenting with ACS. ${ }^{37}$ However, these studies have investigated ACS patients at the time they developed their event. Although it is conceivable that plaque characteristics at the time of a coronary event characterize plaques that are vulnerable to rupture, these studies do not prove that non-invasive plaque imaging can actually predict for future events. Clinical evidence on coronary plaque characteristics subsequently resulting in ACS is very limited. ${ }^{8,38}$ In this study's culprit analysis, we found significant differences in plaque characteristics between plaques that later resulted in ACS and plaques that did not. This observation suits the idea that high risk coronary atherosclerosis has a focal distribution, i.e. it supports the vulnerable plaque theory. ${ }^{39}$ Moreover, we report for the first time that detailed quantification of CAD not only identifies plaque characteristics associated with future ACS, but also provides incremental prognostic value over conventional CCTA reading.

However, these plaque characteristics are not commonly reported in studies, and to our knowledge not regularly examined in clinical practice. Nevertheless, in the US alone, an estimated 3 million cardiac CT procedures are performed every year. It is therefore conceivable that achieving even a moderate increase in prognostic power, such as shown in the present study using semiautomated quantification, may have important implications for clinical risk stratification in stable chest pain patients. In addition, since the health care budgets become more and more strained in many countries, it is essential to maximize the prognostic yield of imaging studies, for instance through improvement of image analysis software.

\section{Reproducibility}

First, we observed a robust reproducibility of the quantification algorithm used in the present study. We report a relatively high inter-observer correlation for total plaque volume, which was however lower for non-calcified plaques and plaque attenuation. This is in concurrence with several publications. ${ }^{29,40}$ In general demarcating the border of non-calcified plaques is more prone to subjectivity of the reader. Klass et al. reported an acceptable inter-observer correlation 
for non-calcified plaques, but higher variability for partially calcified and calcified plaques when using the same quantification algorithm. ${ }^{20}$ These results are somewhat conflicting, potentially explained by the fact that Klass et al. investigated only proximal LAD lesions. In their study, non-calcified plaques were very small while partially calcified and calcified plaques were much larger, possibly resulting in higher variability. Another study utilizing the same quantification algorithm demonstrated a high reproducibility of plaque size and subtype categorization of the observed lesions. ${ }^{19}$

\section{Limitations}

Since this is a retrospective study with a randomly selected control population, we have to be careful in interpreting the difference in plaque characteristics between patients with and without developing ACS. Follow-up was performed using the electronic patient registry of the hospital, therefore we might have missed events occurring outside the hospital. However, since our hospital is the only PCI clinic in the region, and patients were subject to close clinical followup, we expect this number to be very low. Unfortunately, two patients with reported ACS were excluded for this analysis because of non-interpretable CCTA exams. Because our goal was to investigate the potential additional prognostic value of semi-automated plaque quantification, we focused on a specific population with evidence of CAD on their CCTA. Furthermore, the majority of patients with a calcium score $>1000$ were excluded for CCTA. Patients who underwent revascularization were not selected for this study, while they are likely to have a high grade stenosis on CCTA. This might explain the limited prognostic value of calcium score and stenosis severity in our population. Therefore, these results are only applicable for a limited patient population with presence of CAD. Care should be taken when comparing both models (i.e. AUCs), since values of the new model may have been influenced by model overfitting. To further establish the predictive value of the plaque quantification, the proposed cut-off points and models should be tested in an independent, preferably prospective population.

\section{Conclusion}

The semi-automated plaque quantification algorithm identified several parameters predictive for ACS and provided additive predictive value over both clinical risk profiling and conventional CCTA reading. The use of a plaque quantifica- 
tion algorithm can improve risk stratification in stable chest pain patients with coronary artery disease.

\section{References}

1. Roger VL, Go AS, Lloyd-Jones DM, et al. Heart disease and stroke statistics--2012 update: a report from the American Heart Association. Circulation 2012;125:e2-e220.

2. Hoffmann MH, Shi H, Schmitz BL, et al. Noninvasive coronary angiography with multislice computed tomography. JAMA 2005;293:2471-8.

3. Meijboom WB, Meijs MF, Schuijf JD, et al. Diagnostic accuracy of 64-slice computed tomography coronary angiography: a prospective, multicenter, multivendor study. J Am Coll Cardiol 2008;52:2135-44.

4. Hadamitzky M, Freissmuth B, Meyer T, et al. Prognostic value of coronary computed tomographic angiography for prediction of cardiac events in patients with suspected coronary artery disease. JACC Cardiovasc Imaging 2009;2:404-11.

5. Min JK, Dunning A, Lin FY, et al. Age- and sex-related differences in all-cause mortality risk based on coronary computed tomography angiography findings results from the International Multicenter CONFIRM (Coronary CT Angiography Evaluation for Clinical Outcomes: An International Multicenter Registry) of 23,854 patients without known coronary artery disease. J Am Coll Cardiol 2011;58:849-60.

6. Pundziute G, Schuijf JD, Jukema JW, et al. Head-to-head comparison of coronary plaque evaluation between multislice computed tomography and intravascular ultrasound radiofrequency data analysis. JACC Cardiovasc Interv 2008;1:176-82.

7. Motoyama S, Kondo T, Sarai M, et al. Multislice computed tomographic characteristics of coronary lesions in acute coronary syndromes. J Am Coll Cardiol 2007;50:319-26.

8. Motoyama S, Sarai M, Harigaya H, et al. Computed tomographic angiography characteristics of atherosclerotic plaques subsequently resulting in acute coronary syndrome. J Am Coll Cardiol 2009;54:49-57.

9. Voros S, Rinehart S, Qian Z, et al. Prospective validation of standardized, 3-dimensional, quantitative coronary computed tomographic plaque measurements using radiofrequency backscatter intravascular ultrasound as reference standard in intermediate coronary arterial lesions: results from the ATLANTA (assessment of tissue characteristics, lesion morphology, and hemodynamics by angiography with fractional flow reserve, intravascular ultrasound and virtual histology, and noninvasive computed tomography in atherosclerotic plaques) I study. JACC Cardiovasc Interv 2011;4:198-208.

10. Voros S, Rinehart S, Qian Z, et al. Coronary atherosclerosis imaging by coronary CT angiography: current status, correlation with intravascular interrogation and meta-analysis. JACC Cardiovasc Imaging 2011;4:537-48. 
11. Braunwald E, Antman EM, Beasley JW, et al. ACC/AHA 2002 guideline update for the management of patients with unstable angina and non-ST-segment elevation myocardial infarction--summary article: a report of the American College of Cardiology/American Heart Association task force on practice guidelines (Committee on the Management of Patients With Unstable Angina). J Am Coll Cardiol 2002;40:1366-74.

12. D'Agostino RB, Sr., Vasan RS, Pencina MJ, et al. General cardiovascular risk profile for use in primary care: the Framingham Heart Study. Circulation 2008;117:743-53.

13. Versteylen MO, Joosen IA, Shaw LJ, Narula J, Hofstra L. Comparison of Framingham, PROCAM, SCORE, and Diamond Forrester to predict coronary atherosclerosis and cardiovascular events. J Nucl Cardiol 2011;18:904-11.

14. Nieman K, Galema T, Weustink A, et al. Computed tomography versus exercise electrocardiography in patients with stable chest complaints: real-world experiences from a fasttrack chest pain clinic. Heart 2009;95:1669-75.

15. Shrimpton P. Assessment of patient dose in CT: appendix C-European guidelines for multislice computed tomography. European Commission project MSCT: CT safety \& efficacy—a broad perspective. http://www.msct.eu/PDF_FILES/Appendix\%20paediatric\%20CT\%20Dosimetry.pdf Published 2004. Accessed November 20, 2008.

16. Agatston AS, Janowitz WR, Hildner FJ, Zusmer NR, Viamonte M, Jr., Detrano R. Quantification of coronary artery calcium using ultrafast computed tomography. J Am Coll Cardiol 1990;15:827-32.

17. Austen WG, Edwards JE, Frye RL, et al. A reporting system on patients evaluated for coronary artery disease. Report of the Ad Hoc Committee for Grading of Coronary Artery Disease, Council on Cardiovascular Surgery, American Heart Association. Circulation 1975;51:5-40.

18. Min JK, Shaw LJ, Devereux RB, et al. Prognostic value of multidetector coronary computed tomographic angiography for prediction of all-cause mortality. J Am Coll Cardiol 2007;50:1161-70.

19. Korosoglou G, Mueller D, Lehrke S, et al. Quantitative assessment of stenosis severity and atherosclerotic plaque composition using 256-slice computed tomography. Eur Radiol 2010;20:1841-50.

20. Klass O, Kleinhans S, Walker MJ, et al. Coronary plaque imaging with 256-slice multidetector computed tomography: interobserver variability of volumetric lesion parameters with semiautomatic plaque analysis software. Int J Cardiovasc Imaging 2010;26:711-20.

21. Lee MS, Chun EJ, Kim KJ, Kim JA, Vembar M, Choi SI. Reproducibility in the assessment of noncalcified coronary plaque with 256-slice multi-detector CT and automated plaque analysis software. Int J Cardiovasc Imaging 2010;26:237-44.

22. DeLong ER, DeLong DM, Clarke-Pearson DL. Comparing the areas under two or more correlated receiver operating characteristic curves: a nonparametric approach. Biometrics 1988;44:837-45. 
23. Shaw LJ, Raggi P, Schisterman E, Berman DS, Callister TQ. Prognostic value of cardiac risk factors and coronary artery calcium screening for all-cause mortality. Radiology 2003;228:826-33.

24. Hadamitzky M, Distler R, Meyer T, et al. Prognostic value of coronary computed tomographic angiography in comparison with calcium scoring and clinical risk scores. Circ Cardiovasc Imaging 2011;4:16-23.

25. Chow BJ, Small G, Yam Y, et al. Incremental prognostic value of cardiac computed tomography in coronary artery disease using CONFIRM: COroNary computed tomography angiography evaluation for clinical outcomes: an InteRnational Multicenter registry. Circ Cardiovasc Imaging 2011;4:463-72.

26. Russo V, Zavalloni A, Bacchi Reggiani ML, et al. Incremental prognostic value of coronary CT angiography in patients with suspected coronary artery disease. Circ Cardiovasc Imaging 2010;3:351-9.

27. Hur J, Kim YJ, Lee HJ, et al. Quantification and characterization of obstructive coronary plaques using 64-slice computed tomography: a comparison with intravascular ultrasound. J Comput Assist Tomogr 2009;33:186-92.

28. Sun J, Zhang Z, Lu B, et al. Identification and quantification of coronary atherosclerotic plaques: a comparison of 64-MDCT and intravascular ultrasound. AJR Am J Roentgenol 2008;190:748-54.

29. Leber AW, Becker A, Knez A, et al. Accuracy of 64-slice computed tomography to classify and quantify plaque volumes in the proximal coronary system: a comparative study using intravascular ultrasound. J Am Coll Cardiol 2006;47:672-7.

30. Stone GW, Maehara A, Lansky AJ, et al. A prospective natural-history study of coronary atherosclerosis. N Engl J Med 2011;364:226-35.

31. Pundziute G, Schuijf JD, Jukema JW, et al. Evaluation of plaque characteristics in acute coronary syndromes: non-invasive assessment with multi-slice computed tomography and invasive evaluation with intravascular ultrasound radiofrequency data analysis. Eur Heart J 2008;29:2373-81.

32. Schuijf JD, Beck T, Burgstahler C, et al. Differences in plaque composition and distribution in stable coronary artery disease versus acute coronary syndromes; non-invasive evaluation with multi-slice computed tomography. Acute Card Care 2007;9:48-53.

33. Hoffmann U, Moselewski F, Nieman K, et al. Noninvasive assessment of plaque morphology and composition in culprit and stable lesions in acute coronary syndrome and stable lesions in stable angina by multidetector computed tomography. J Am Coll Cardiol 2006;47:1655-62.

34. Hammer-Hansen S, Kofoed KF, Kelbaek H, et al. Volumetric evaluation of coronary plaque in patients presenting with acute myocardial infarction or stable angina pectoris-a multislice computerized tomography study. Am Heart J 2009;157:481-7. 
35. Sato A, Ohigashi H, Nozato T, et al. Coronary artery spatial distribution, morphology, and composition of nonculprit coronary plaques by 64-slice computed tomographic angiography in patients with acute myocardial infarction. Am J Cardiol 2010;105:930-5.

36. Kitagawa T, Yamamoto H, Horiguchi J, et al. Characterization of noncalcified coronary plaques and identification of culprit lesions in patients with acute coronary syndrome by 64-slice computed tomography. JACC Cardiovasc Imaging 2009;2:153-60.

37. Pflederer T, Marwan M, Schepis T, et al. Characterization of culprit lesions in acute coronary syndromes using coronary dual-source CT angiography. Atherosclerosis 2010;211:43744.

38. Matsumoto N, Sato Y, Yoda S, et al. Prognostic value of non-obstructive CT low-dense coronary artery plaques detected by multislice computed tomography. Circ J 2007;71:1898903.

39. Cheruvu PK, Finn AV, Gardner C, et al. Frequency and distribution of thin-cap fibroatheroma and ruptured plaques in human coronary arteries: a pathologic study. J Am Coll Cardiol 2007;50:940-9.

40. Knollmann F, Ducke F, Krist L, et al. Quantification of atherosclerotic coronary plaque components by submillimeter computed tomography. Int J Cardiovasc Imaging 2008; 24:301-10. 


\title{
Editorial
}

\section{Visualizing Vulnerability Toward a New Cardiac Score}

\author{
Edward P. Shapiro, David E. Bush
}

Johns Hopkins University School of Medicine, Division of

Cardiology, Baltimore, Maryland, United States of America.

J Am Coll Cardiol. 2013; 61(22): 2306-8. 
Coronary artery disease is almost ubiquitous in our population but comes in a wide variety of shapes and sizes. For decades, a simple but powerful concept has guided decision making in prescribing therapies: the highest risk forms of the disease require the most aggressive treatments, while the lowest risk forms warrant only the most gentle. That is, the patient with three vessel disease and LV dysfunction requires bypass surgery, while the one with minimal coronary irregularities requires only a statin or aspirin. Even within the context of patients receiving medical therapy without revascularization, a wide range of treatments are now or will soon be available, including aspirin, mild or strong P2Y12 inhibitors, statins and PCSK9 inhibitors to moderate or low LDL targets, interventions to augment HDL, anti-hypertensives to various levels of BP reduction, ACE inhibitors and ARBs, and drugs to inhibit vascular inflammation. To prescribe individualized therapy we need an accurate assessment of risk. The study by Versteylen et $\mathrm{al}^{1}$ in the current edition of JACC demonstrates that quantitative information derived from the direct imaging of atherosclerotic lesions by CT angiography has potential to define risk more accurately and therefore guide therapy in a way that has previously not been possible.

\section{Vulnerable plaque.}

A large literature has accumulated in which characteristics of an individual plaque susceptible to rupture or erosion ${ }^{2}$ with subsequent thrombus formation are defined, with the thought that these plaques might be detected by imaging methods and treated by catheter-based therapy before they precipitate an ischemic event. At autopsy, histologic characteristics of such vulnerable plaques, called thin-cap fibroatheromas (TCFA), include the presence of a large necrotic core and a thin ( $<65$ micron) fibrous cap weakened by infiltration with macrophages $^{2}$. During life, TCFAs may be detected invasively using virtual histology intravascular ultrasound (VH-IVUS), which displays them as plaques with lipid adjacent to the lumen. The PROSPECT study determined that plaques with the VH-TCFA pattern, plaque burden exceeding $70 \%$, and maximal luminal area $<4 \mathrm{~mm}^{2}$ were most likely to lead to events. Other invasive techniques are under development and study, such as near-infrared spectroscopy ${ }^{3}$, in which the arterial wall is interrogated for lipid deposits, and optical coherence tomography ${ }^{4}$, in which reflected light generates high-resolution images of superficial layers of the vessel wall. Non-invasively, by CTA, visualized vulnerable features include low-attenuation (Hounsfield unit <30) sometimes in a "signet ring pattern"5, positive remodeling, and spotty calcification ${ }^{6}$. Yet, it is unlikely that any ap- 
proach based on the detection of individual vulnerable plaques will bear clinical fruit in the foreseeable future ${ }^{7}$. Most plaque disruptions are silent events. Even if thrombus forms, in most cases there is no luminal obstruction ${ }^{8-10}$ and no ischemia, and the disruption merely results in unnoticed plaque growth. Furthermore, serial study by IVUS suggests that non-calcified plaque with vulnerable characteristics by VH-IVUS may evolve into a more stable appearance and vice versa, even in a period as short as 12 months ${ }^{11}$. Therefore, even if new imaging techniques are successful in more accurately identifying plaques that may rupture, any attempt at catheter-based therapy would need to be applied to many, many sites before a single event is prevented.

\section{Vulnerable patient.}

The solution is to consider the vulnerability of the entire patient rather than that of the individual plaque. One might then take into account susceptibility to thrombosis 7,12 . In addition, the obvious other factor to consider is the total quantity of high-risk plaque material, which determines the probability that plaque disruption will occur at some unknown site or other. Ideally, there would be a global vulnerable plaque "score" analogous to the calcium score, but sensitive to the plaque characteristics that are directly responsible for ischemic events rather than calcium, an indirect marker of disease activity. To have the greatest impact, our efforts at defining vulnerability should be focused on finding the patient with a very high plaque score so that systemic rather than local therapy of appropriate aggressiveness can be applied. The high scoring patient would need the strictest LDL and BP management, the strongest antiplatelet regimen, and the broadest blockade of the renin angiotensin system. Future therapies that are successful at reducing residual risk with treatment directed at $\mathrm{HDL}^{13}$ and suppression of inflammation ${ }^{14}$, might also be heaped upon this unfortunate patient. The patient with a low score might be adequately protected with an aspirin alone. Versteylen et $\mathrm{al}^{1}$ have made progress toward this personalization of risk assessment by following 1650 patients with stable chest pain who had undergone cardiac CTA, for an average of 26 months and comparing the 26 patients who developed acute coronary syndrome with 101 controls with CAD randomly selected from the non-ACS group. Coronary arteries were evaluated with a whole-heart quantitative technique, using a commercially available software package that draws contours along plaque of different densities and allows quantification of global and maximal plaque characteristics. Several similar packages are on the market and have been validated by 
comparison to IVUS ${ }^{15,16}$. The authors found that the most predictive global parameters were the total volume of non-calcified plaque, the total volume of all plaque, and the total number of plaques. The most predictive local parameters were the presence of plaque with the highest volume, and lesions with the highest plaque burden. Receiving honorable mention were plaques with the highest area, the highest non-calcified percentage, the lowest attenuation, and the highest remodeling index. These findings are consistent with the wellaccepted concept that non-calcified plaque is the usual culprit and that calcified plaque is only a marker of disease burden and not a major player in the mechanism of ischemic events. This work confirms and extends the findings of Kristensen et $a l^{17}$, who showed that in post-ACS patients (a higher risk population than that studied here) multivariate analysis yielded the same two parameters as the major determinants of prognosis: non-calcified plaque volume and total plaque volume. Therefore, it appears that elements of a new scoring system should certainly include total volume of non-calcified plaque and maximal volume or plaque burden of an individual plaque. One new contribution of Versteylen et al is the finding that these parameters can be measured semiautomatically, which brings an otherwise time-consuming measurement one step closer to clinical utility. The authors did not describe the average time to make and edit these measurements. The software that requires the least human correction to correspond with a visual assessment, and which provides tools for the fastest editing, will prevail here. Conventional interpretation of CT angiography includes calcium scoring, a description of plaques and their characteristics, and an assessment of stenosis with designation as non-obstructive, one, two, or three vessel disease ${ }^{18}$. Versteylen et al here demonstrate that this new type of quantitative analysis provides prognostic information that improves upon conventional interpretation of CT angiography: the area under the receiver operating characteristics curve for predicting events was increased from 0.64 to 0.79 when these new quantitative parameters were applied. These data suggest that the multiple studies showing accurate prediction of prognosis by cardiac CT using conventional interpretation ${ }^{19-22}$ have not yet fully tapped the power of the technique. For example, the CONFIRM international registry has provided multicenter prospective data in 24,775 patients, showing decreasing survival in non-obstructive vs. 1 vessel obstructive, vs. 2 vessel obstructive, vs. 3 vessel obstructive disease, an analysis relying on old fashioned "lumenography" rather than upon plaque within the arterial wall23. Although a semiquantitative "segment involvement score (SIS)" also was reported, a more 
quantitative assessment of non-calcified plaque in that and other large datasets is likely to provide new insights. Such an analysis of the CONFIRM data is underway.

\section{CAC vs plaque scoring.}

The availability of calcium scoring has greatly refined our ability to predict risk in the asymptomatic population and it now carries a IIa guideline recommendation for patients at intermediate Framingham risk, in which half can be correctly re-classified into high or low categories ${ }^{24}$. The guidelines give a IIb recommendation for those at low to moderate risk. Now that CT angiography can be performed with a radiation dose comparable to that of calcium scoring 25 , would a risk score based on non-calcified plaque provide better classification of asymptomatic patients? Choi ${ }^{26}$ followed 1000 asymptomatic patients and did not find an advantage of CT angiography over CAC for screening, but theirs was a very low risk population and only 15 events including only one ACS occurred in the 17 months of follow-up reported. Recent findings from the CONFIRM registry also suggest no advantage of CT angiography over CAC in 7590 subjects from this population ${ }^{27}$ but this was a conventional CT analysis that did not include non-calcified plaque volumes or other global parameters introduced more recently. Perhaps this result would change if the data were reanalyzed with these measurements included. However, from a practical point of view, the simplicity, speed, and low cost of calcium imaging will make it hard to beat as a screening tool for low risk populations. It is in higher risk populations, such as patients with stable angina, where CT angiography with conventional interpretation is already known to predict adverse outcomes ${ }^{19-22,28}$ and in post-ACS patients, where the augmented power of plaque-scored CTA may become evident. Calcium scoring has little role in these populations.

\section{Resolving some controversies in cardiology.}

Availability of a plaque score may resolve some fundamental contradictions in cardiology. For instance, we clinicians pay lip service to the fact that most ischemic events result from distuption of non-stenotic plaques ${ }^{12}$, yet we manage patients based on the presence of single, double or triple vessel stenotic disease. The true importance of stenosis vs. plaque volume is unknown. While it is true that CT studies ${ }^{20}$ have confirmed catheterization studies showing a major difference in prognosis between nonobstructive, single, double and triple vessel disease, multi-vessel patients have more nonobstructive plaque as well, which 
could be the real determinant of prognosis. An outcome study reporting both plaque measures and stenosis could reveal whether one or both aspects of the disease have an independent prognostic effect. Another issue needing clarification is how to select the optimal method for revascularization when it is deemed to be necessary. We currently decide whether to use PCI vs. CABG based on technical features, the anticipated likelihood of restenosis, the angiographic load of disease, and other factors captured by the SYNTAX score ${ }^{29}$. Wouldn't it make more sense to stent arteries in which the atherosclerosis is truly focal, and bypass those with an extensive longitudinal extent of plaque that could rupture anywhere along the vessel, thereby treating the whole artery? That is, a revised SYNTAX score including the vessel plaque volume might improve the selection process. Finally, research studies of new drugs or procedures to treat coronary artery disease would require far fewer patients if they were directed at a select group with a predictable high incidence of acute coronary syndrome.

\section{Conclusion.}

Studies like that of Versteylen et al, in which the diffuse nature of coronary atherosclerosis is recognized and measured, lay the groundwork for development of a new and more logical way to predict the risk of coronary events. The prescription of personalized, targeted treatment based on CT imaging is a promising direction. Large well-designed studies will be necessary to learn how to best use this new information.

\section{References}

1. Versteylen MO, Kietselaer B, Dagnelie P, et al. Additive value of semi-automated quantification of coronary artery disease using cardiac CT-angiography to predict for future acute coronary syndrome. J Am Coll Cardiol 2013; 61(22): 2296-2305.

2. Finn AV, Nakano M, Narula J, Kolodgie FD, Virmani R. Concept of vulnerable/unstable plaque. Arterioscler Thromb Vasc Biol. 2010;30:1282-92.

3. Waxman S, Dixon SR, L'Allier P, et al. In vivo validation of a catheter- based near-infrared spectroscopy system for detection of lipid core coronary plaques: Initial results of the SPECTACL study. JACC Cardiovasc Imaging. 2009;2:858-68.

4. Regar E, Ligthart J, Bruining N, van Soest G. The diagnostic value of intracoronary optical coherence tomography. Herz. 2011;36:417-29. 
5. Seifarth H, Schlett CL, Nakano M, et al. Histopathological correlates of the napkin-ring sign plaque in coronary CT angiography. Atherosclerosis. 2012;224:90-6.

6. Motoyama S, Sarai M, Harigaya H, et al. Computed tomographic angiography characteristics of atherosclerotic plaques subsequently resulting in acute coronary syndrome. J Am Coll Cardiol. 2009;54:49-57.

7. Arbab-Zadeh A, Nakano M, Virmani R, Fuster V. Acute coronary events. Circulation. 2012;125:1147-56.

8. Arbustini E, Grasso M, Diegoli M, et al. Coronary atherosclerotic plaques with and without thrombus in ischemic heart syndromes: A morphologic, immunohistochemical, and biochemical study. Am J Cardiol. 1991;68:36B-50B.

9. Sato Y, Hatakeyama K, Marutsuka K, Asada Y. Incidence of asymptomatic coronary thrombosis and plaque disruption: Comparison of non-cardiac and cardiac deaths among autopsy cases. Thromb Res. 2009;124:19-23.

10. Wang Z, Inami S, Kirinoki S, et al. Angioscopic study of silent plaque disruption in nonischemic related coronary artery in patients with stable ischemic heart disease. Int Heart J. 2010;51:383-7.

11. Kubo T, Maehara A, Mintz GS, et al. The dynamic nature of coronary artery lesion morphology assessed by serial virtual histology intravascular ultrasound tissue characterization. J Am Coll Cardiol. 2010;55:1590-7.

12. Naghavi M, Libby P, Falk E, et al. From vulnerable plaque to vulnerable patient: A call for new definitions and risk assessment strategies: Part II. Circulation. 2003;108:1772-8.

13. Joy TR. Novel HDL-based therapeutic agents. Pharmacol Ther. 2012;135:18-30.

14. Coomes E, Chan ES, Reiss AB. Methotrexate in atherogenesis and cholesterol metabolism. Cholesterol. 2011;2011:503028.

15. Rinehart S, Vazquez G, Qian Z, Murrieta L, Christian K, Voros S. Quantitative measurements of coronary arterial stenosis, plaque geometry, and composition are highly reproducible with a standardized coronary arterial computed tomographic approach in high-quality CT datasets. J Cardiovasc Comput Tomogr. 2011;5:35-43.

16. Boogers MJ, Broersen A, van Velzen JE, et al. Automated quantification of coronary plaque with computed tomography: Comparison with intravascular ultrasound using a dedicated registration algorithm for fusion-based quantification. Eur Heart J. 2012;33:1007-16.

17. Kristensen TS, Kofoed KF, Kuhl JT, Nielsen WB, Nielsen MB, Kelbaek H. Prognostic implications of nonobstructive coronary plaques in patients with non-ST-segment elevation myocardial infarction: A multidetector computed tomography study. J Am Coll Cardiol. 2011;58:502-9.

18. Raff GL, Abidov A, Achenbach S, et al. SCCT guidelines for the interpretation and reporting of coronary computed tomographic angiography. J Cardiovasc Comput Tomogr. 2009;3:122-36. 
19. Min JK, Shaw LJ, Devereux RB, et al. Prognostic value of multidetector coronary computed tomographic angiography for prediction of all-cause mortality. J Am Coll Cardiol. 2007;50:1161-70.

20. Min JK, Dunning A, Lin FY, et al. Age- and sex-related differences in all-cause mortality risk based on coronary computed tomography angiography findings results from the international multicenter CONFIRM (coronary CT angiography evaluation for clinical outcomes: An international multicenter registry) of 23,854 patients without known coronary artery disease. J Am Coll Cardiol. 2011;58:849-60.

21. Hadamitzky M, Freissmuth B, Meyer T, et al. Prognostic value of coronary computed tomographic angiography for prediction of cardiac events in patients with suspected coronary artery disease. JACC Cardiovasc Imaging. 2009;2:404-11.

22. Kwon SW, Kim YJ, Shim J, et al. Coronary artery calcium scoring does not add prognostic value to standard 64-section CT angiography protocol in low-risk patients suspected of having coronary artery disease. Radiology. 2011;259:92-9.

23. Otaki Y, Arsanjani R, Gransar H, et al. What have we learned from CONFIRM? Prognostic implications from a prospective multicenter international observational cohort study of consecutive patients undergoing coronary computed tomographic angiography. J Nucl Cardiol. 2012;19:787-95.

24. Elias-Smale SE, Proenca RV, Koller MT, et al. Coronary calcium score improves classification of coronary heart disease risk in the elderly: The rotterdam study. J Am Coll Cardiol. 2010;56:1407-14. 


\section{Chapter}

\section{Chemokine Ligands in Patients presenting with Stable Chest Pain: Association with Atherosclerosis and Future Cardiovascular Events}

Mathijs O. Versteylen, Marco Manca, Ivo A. Joosen, David E. Schmidt, Marco Das, Leonard Hofstra, Harry J. Crijns, Erik A. Biessen, Bas L. Kietselaer

Submitted 


\section{ABSTRACT}

Background Serum levels of particular CC chemokine ligands (CCLs), such as CCL3-5-18, are elevated during acute coronary syndrome (ACS) and correlate with secondary events. Their strong expression in plaque as well as functional involvement in plaque inflammation led us to investigate whether CCL3-5-18 are linked to the extent of coronary artery disease (CAD) and prognostic for primary events during follow-up.

Methods We measured CCL3-5-18 serum concentrations in 712 patients with chest discomfort referred for cardiac CT angiography (CCTA). Obstructive CAD was defined as $\geq 50 \%$ stenoses. Extent of CAD was measured by calcium score (Agatston) and segment involvement score (number of coronary segments with any CAD, range 0-16). Patients were followed-up for all-cause mortality, ACS and revascularization procedures, for a mean $26 \pm 7$ months.

Results Mean age of the population was 56 \pm 11 years, 398 (56\%) were males. Patients with obstructive CAD had significantly higher CCL5 ( $\mathrm{p}=0.02)$, and borderline significantly elevated CCL18 plasma levels as compared to patients with $<50 \%$ stenosis $(\mathrm{p}=0.06)$. CCL3 concentrations did not differ. CCL18 levels were associated with coronary calcification $(\mathrm{p}=0.002)$ and segment involvement score $(\mathrm{p}=0.007)$. When corrected for traditional risk factors, CCL5 but not CCL3 and CCL18, provided independent predictive value for obstructive CAD, at an odds ratio (OR) 1.27 (1.02-1.59), p=0.04. In addition, CCL5 provided independent predictive value for primary events during follow-up, OR 1.62 (1.03-2.57), $\mathrm{p}=0.04$.

Conclusions While CCL18 serum levels correlated with extent of CAD including calcification, CCL5 demonstrated an independent association with the presence of obstructive CAD, and occurrence of primary cardiac events. 


\section{INTRODUCTION}

Atherosclerosis is generally regarded as a dyslipidemic disorder with a strong inflammatory character. ${ }^{1}$ This inflammation process is in part directed by chemokines, which play a role in mediating leukocyte recruitment to sites of injury, vascular smooth muscle cell proliferation, neovascularization and platelet activation. ${ }^{2,3}$ Among others, CC chemokine ligand (CCL) 3, 5 and 18 have been detected in human atherosclerotic lesions. ${ }^{4,5}$

In addition, CCL3, 5 and 18 have been associated with acute coronary syndrome (ACS) and refractory unstable angina pectoris (UAP) in several prospective studies. In patients with UAP, CCL5 and 18 were transiently raised during UAP and indicative of refractory syndromes. ${ }^{6}$ CCL3 levels were significantly elevated in patients with acute myocardial infarction (AMI) as compared to controls, as well as transiently elevated in UAP patients. In addition CCL3 elevation was associated with future ACS. ${ }^{7}$ Also, in a larger cohort of patients presenting with ACS, CCL3, 5 and 18 were independently associated with fatal events during follow-up. ${ }^{8}$ In addition, macrophage expression of CCL18 in patients with atherosclerosis was significantly higher as compared with controls, suggesting CCL18 has a role in atherosclerotic plaque formation. ${ }^{9}$

Importantly, all studies so far were done on patients presenting with ACS (AMI or UAP), consequently investigating associations of CCL concentrations with secondary events. However, the potential of CCLs to predict for primary events is unknown. Although CCL3, 5 and 18 might contribute to plaque formation, and could therefore represent attractive therapeutic targets, their association with presence and extent of coronary artery disease (CAD) is not well understood. We hypothesized that CCL3, 5 and 18 are associated with both CAD as well as cardiac events in stable chest pain patients.

The goal of this study was therefore to address the relationship between CCL3, 5 and 18 and the presence and extent of CAD as defined by CCTA in a cohort of stable chest pain patients. In addition, the association of CCL3, 5 and 18 with primary cardiac events during follow-up was investigated. 


\section{METHODS}

\section{Study population}

We analyzed a cohort of patients who were referred from the outpatient clinic for CCTA to rule out CAD. All CCTAs were performed between January 2008 and June 2010. Included were patients who underwent both a calcium score scan as well as CT-angiography. Our protocol precluded CCTA when calcium score was $>1000$. CCTA was also not performed in patients with unstable angina, hemodynamic instability, renal insufficiency, severe iodine allergy and pregnancy.

In total 1,096 patients were scanned. Excluded were 117 patients who had an inconclusive CCTA. Another 95 patients were excluded because of missing clinical data. Additionally, 172 blood samples were hemolytic and were excluded for this analysis. Eventually, 712 were subject of this study and plasma was screened for CCL3, 5 and 18 levels. All patients signed informed consent. The study protocol conforms to the ethical guidelines of the 1975 Declaration of Helsinki, as reflected in an a priori approval by the Institutional Review Board and ethics committee.

\section{CCTA acquisition}

CCTA was performed as previously described. ${ }^{10}$ using a 64-slice multi detectorrow computed tomography scanner (Brilliance 64; Philips Healthcare, Best, The Netherlands) with a $64 \times 0.625 \mathrm{~mm}$ slice collimation and a rotation time of 420 ms. The tube voltage was 80 or $120 \mathrm{kV}$ and the tube current varied from 150 to $1000 \mathrm{mAs}$, depending on the patient's height and weight. Patients received a contrast bolus (Xenetix 350, Guerbet) of 85-110 ml, injected at a rate of 5.0-7.2 $\mathrm{ml} / \mathrm{s}$. In patients with heart rates $<65$ beats per minute (bpm), a prospective ECG-triggered axial scan was performed, in patients with heart rates $>65 \mathrm{bpm}$, a retrospective ECG-triggered spiral acquisition protocol was used. In addition, a native scan using $120 \mathrm{kV}$ and $3 \mathrm{~mm}$ slice thickness was performed to determine the coronary calcium score (Agatston score). Mean total estimated radiation dose was calculated by multiplying the dose-length product by the conversion factor of $0.014 \mathrm{mSv} / \mathrm{mGy} / \mathrm{cm}$ for the thorax. ${ }^{11}$ 


\section{CCTA assessment}

Agatston method was used to quantify coronary calcium score. ${ }^{12}$ CT angiograms were independently analyzed by a cardiologist and a radiologist, both experienced in reading cardiac CT and blinded to clinical information, using the American Heart Association 16 coronary segments model. ${ }^{13}$ In the case of disagreement, consensus was reached by discussion between the two readers. The coronary artery tree was assessed using the source images on the provided software (Comprehensive Cardiac, Philips Healthcare). The degree of stenosis was defined visually and a lesion severity score was calculated as follows: no lesion (score 0 ), mild diameter stenosis $(<50 \%$, score 1$)$, moderate $(50-70 \%$, score 2$)$ and severe diameter stenosis $(>70 \%$, score 3$)$. This resulted in a lesion severity score ranging from 0-3 for every individual patient. The presence of one or more lesions with a $\geq 50 \%$ stenosis was regarded as significant CAD. In addition, the segment involvement score was defined as the total number of coronary segments with detectable CAD (range $0-16) .{ }^{14}$

\section{Biomarker assessment}

Blood samples were collected by ethylenediaminetetraacetic acid (EDTA) coated Vacutainer venipuncture just before the scan. Samples were kept at 4 degrees Celsius and centrifuged at 4,100 rpm for 10 minutes, and then stored at $80^{\circ} \mathrm{C}$ until analysis, all within 2 hours. Total cholesterol, HDL, triglycerides, glucose, and creatinine concentrations were measured using the Synchron LX20 (Beckman Coulter Inc., Brea, CA, USA). Low-density lipoprotein (LDL) was calculated using the Friedewald equation ${ }^{15}$ except for subjects with triglycerides $>400 \mathrm{mg} / \mathrm{dL}$ and total cholesterol $<50 \mathrm{mg} / \mathrm{dL}$, in which case LDL was determined on the Cobas Mira Plus (Roche Diagnostics, Basel, Switzerland).

\section{CCL assessment}

Baseline blood specimens were collected from storage in July 2012. The sera were snap frozen and stored at $-70^{\circ} \mathrm{C}$ until further analyses and underwent only one freeze-thawing cycle. Baseline serum levels of CCL3/MIP- $1 \alpha$, CCL5/RANTES and CCL18/PARC were determined by commercially available ELISA kits from R\&D systems (Abingdon, United Kingdom; Human CCL3/MIP-1 alpha Quantikine ELISA Kit, $\leq 8.9 \%$ intra-assay variation, $\mathrm{Hu}-$ man CCL 5/RANTES DuoSet and Human CCL18/PARC DuoSet). CCL3 ELISA was performed according to the manufacturer's instructions. For CCL5 
measurements, $0.75 \mu \mathrm{g} / \mathrm{mL}$ capture antibody and $10 \mathrm{ng} / \mathrm{mL}$ detection antibody were used, for CCL18 we used $1.5 \mu \mathrm{g} / \mathrm{mL}$ capture antibody and $200 \mathrm{ng} / \mathrm{mL}$ detection antibody.

\section{Follow-up}

Patients were followed up for the occurrence of all-cause mortality, acute coronary syndrome (ACS) (including myocardial infarction) and revascularization procedures based on conventional coronary angiography including percutaneous coronary intervention (PCI) and coronary artery bypass grafting (CABG), for a mean $26 \pm 7$ months. Electronic patient records were checked, ACS was defined as typical angina pectoris and troponin $\mathrm{T}$ elevation $(>0.01 \mu \mathrm{g} / \mathrm{L})$ and $\mathrm{ST}$ segment elevation/depression of $\geq 1 \mathrm{~mm}$, or at least two of these symptoms together with invasive angiographic confirmation of a culprit lesion. ${ }^{16}$ Further, we censored follow-up after the first endpoint, so that the recorded ACS was not a complication of revascularization therapy. Patients were seen by their cardiologist on a regular basis, and all hospital visits were recorded in the electronic patient records. Figure 1 shows patient selection and follow-up.

Figure 1. Flow logistic diagram

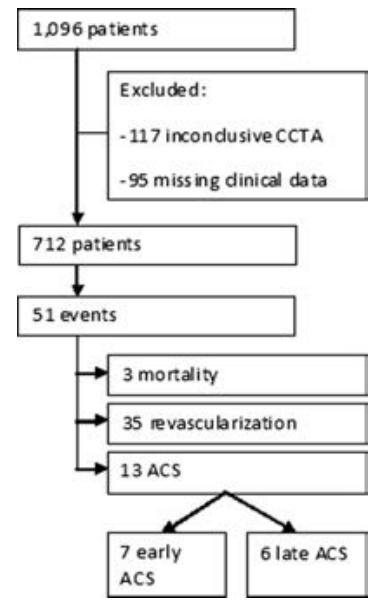

\section{Statistics}

Data were analyzed using SPSS 19.0 (SPSS Inc., Chicago, IL, USA). Continuous variables were presented as mean \pm standard deviation (SD) or median and interquartile range (IQR), whether they were of normal or nonparametric distribution, respectively. Normality of data distribution was evaluated using the Kolmogorov-Smirnov test. Differences were assessed using student's t-test for normally distributed data, or Kruskal Wallis test for nonparametric distributions. Proportions (\%) were used for categorical values, in which differences were assessed using Fisher's exact test.

In order to assess the association of individual CCL values with the extent of CAD, linear regression models were calculated using calcium score and segment involvement score as the dependent variable. Base-10 logarithmic trans- 
formation was used in order to include continuous variables with a non-normal distribution. Also, logistic regression models were calculated using presence of $\geq 50 \%$ stenosis on CCTA as the dependent variable. When appropriate, multivariable analysis was performed, correcting for age and gender (model 1) and for age, gender, diabetes mellitus, body mass index (BMI), smoking, family history, systolic blood pressure and total cholesterol (model 2).

In addition, multivariable logistic regression models incorporating the presence of a $\geq 50 \%$ coronary stenosis (model 3 ) or of the segment involvement score (model 4) were applied to evaluate the association of CCLs with the occurrence of cardiac events during follow-up. For tested CCLs that showed independent predictive value, receiver operating characteristic (ROC) curves were plotted to assess the additional predictive value over clinical risk factors. To predict for $\geq 50 \%$ coronary stenosis, we assessed two incremental models; 1) clinical risk factors, 2) clinical risk factors + CCL value. The same models were plotted to predict for events. Area under the curves (AUC) were produced to evaluate the incremental value of the combined approach and differences between AUCs were tested for significance. ${ }^{17}$ All p-values were 2-sided, and a p-value below 0.05 was considered statistically significant.

\section{RESULTS}

\section{Study population}

Mean age of the study population was $56 \pm 11$ years, 398 (56\%) were males. Further, 169 (24\%) were smokers, $58(8 \%)$ had diabetes mellitus. The indication for CCTA was typical chest pain in $88(12 \%)$, atypical in $310(44 \%)$ and nonanginal complaints in $314(44 \%)$. Mean radiation dose was $5.6 \pm 4.5 \mathrm{mSv}$. The median concentration of CCL3 was $2.7(0.0-7.0) \mathrm{pg} / \mathrm{mL}$, of CCL5 it was 3,614 $(1,547-8,360) \mathrm{pg} / \mathrm{mL}$ and for CCL18 it was 78,553 $(45,906-126,716) \mathrm{pg} / \mathrm{mL}$. Baseline characteristics are further described in table 1. 
Table 1. Baseline characteristics

\begin{tabular}{lccc} 
& $<50 \%$ stenosis & $\geq 50 \%$ stenosis & $\mathrm{p}$-value \\
& $\mathrm{n}=509$ & $\mathrm{n}=203$ & \\
\hline Age (yrs) & $54.7 \pm 10.2$ & $60.9 \pm 9.3$ & $<0.001$ \\
Male gender & $266(52.3 \%)$ & $132(65.0 \%)$ & 0.002 \\
Smoking & $107(21.0 \%)$ & $62(30.5 \%)$ & 0.008 \\
Diabetes Mellitus & $41(8.1 \%)$ & $17(8.4 \%)$ & 0.88 \\
Family history & $187(36.7 \%)$ & $85(41.9 \%)$ & 0.23 \\
Systolic blood pressure $(\mathrm{mmHg})$ & $140 \pm 17$ & $147 \pm 20$ & $<0.001$ \\
BMI $\left(\mathrm{kg} / \mathrm{m}^{2}\right)$ & $26.9 \pm 4.1$ & $27.4 \pm 4.4$ & 0.14 \\
Total cholesterol (mmol/L) & $5.4 \pm 1.1$ & $5.4 \pm 1.3$ & 0.83 \\
Typical chest pain & $59(11.6 \%)$ & $29(14.3 \%)$ & 0.32 \\
Calcium score & $0(0-24.5)$ & $201(53.5-455)$ & $<0.001$ \\
Involvement score & $0(0-2)$ & $5(3-7)$ & $<0.001$ \\
CCL3 $\left(\mathrm{pg} / \mathrm{mL}^{*}\right.$ & $2.5(0.0-7.0)$ & $2.8(0.4-7.0)$ & 0.37 \\
CCL5 $\left(\mathrm{pg} / \mathrm{mL}^{*} 10^{2}\right)$ & $33.4(14.5-77.0)$ & $40.7(18.5-109.1)$ & 0.02 \\
CCL18 $\left(\mathrm{pg} / \mathrm{mL}^{* 10^{2}}\right)$ & $747(451-1241)$ & $885(503-1332)$ & 0.06 \\
\hline
\end{tabular}

Values are presented as numbers (\%), means \pm standard deviation or medians (IQR). Significant pvalues are in bold. $\mathrm{BMI}=$ body mass index, $\mathrm{CCL}=\mathrm{CC}$ chemokine ligand.

\section{Association CCL with CAD}

Serum concentration of CCL5 was significantly, and that of CCL18 borderline significantly higher in patients with $\geq 50 \%$ coronary stenosis, as compared with patients with $<50 \%$ stenosis (table 1). The association of CCL3, 5 and 18 with the severity of CAD as reflected by lesion severity score is presented in figure 2 . More severe CAD was associated with trend toward higher CCL5 $(\mathrm{p}=0.07)$ and CCL18 concentrations $(\mathrm{p}=0.01)$. In addition, especially CCL18 was associated with the extent of $\mathrm{CAD}$, as reflected by the calcium score (table 2) and the segment involvement score (table 3). As is shown in table 4, only CCL5 provided independent information to predict for a $\geq 50 \%$ coronary stenosis when corrected for traditional risk factors. However, none of the CCLs was an independent predictor for calcium score or involvement score, not when corrected for age and gender, or a combination of all risk factors. 
Figure 2. CCL concentrations for different degrees of CAD
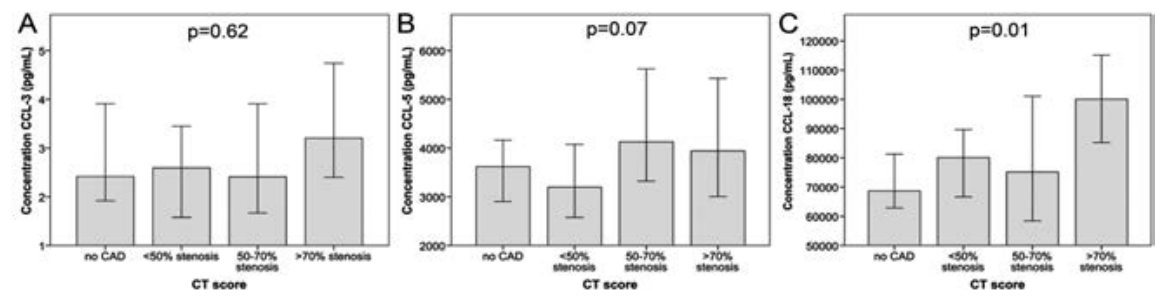

Median concentrations of CCLs for patients without CAD, $<50 \%$ stenosis, $50-70 \%$ stenosis and $\geq 50 \%$ stenosis, respectively. For CCL3 (panel A) there was no significant trend in concentration and higher stenosis grade, $\mathrm{p}=0.62$. However, for CCL5 (panel B), there was a trend towards significantly higher concentration in patients with higher stenosis grades, $\mathrm{p}=0.07$. For CCL18 (panel C), there was a significant trend towards higher concentrations for patients with a higher stenosis present on CCTA, $\mathrm{p}=0.01$.

Table 2. Univariable regression analysis to predict for coronary calcium score (log-transformed)

\begin{tabular}{lccc} 
& B-value & $95 \%$ CI & p-value \\
\hline Age (yrs) & 0.44 & $0.04-0.05$ & $<0.001$ \\
Male gender & 0.14 & $0.15-0.47$ & $<0.001$ \\
Smoking & 0.05 & $-0.05-0.33$ & 0.15 \\
Diabetes Mellitus & 0.10 & $0.12-0.70$ & 0.006 \\
Family history & 0.01 & $-0.14-0.20$ & 0.72 \\
Systolic blood pressure (mm Hg) & 0.23 & $0.01-0.02$ & $<0.001$ \\
BMI (kg/m ${ }^{2}$ ) & 0.06 & $-0.00-0.04$ & 0.09 \\
Total cholesterol (mmol/L) & -0.05 & $-0.12-0.02$ & 0.16 \\
Typical chest pain & -0.01 & $-0.28-0.20$ & 0.75 \\
Lg10 CCL3 & 0.04 & $-0.09-0.28$ & 0.33 \\
Lg10 CCL5 & -0.02 & $-0.11-0.07$ & 0.60 \\
Lg10 CCL18 & 0.12 & $0.16-0.73$ & 0.002 \\
\hline
\end{tabular}

Univariable linear regression model. $\mathrm{BMI}=$ body mass index, $\mathrm{CCL}=\mathrm{CC}$ chemokine ligand. 
Table 3. Univariable regression analysis to predict for involvement score (log-transformed)

\begin{tabular}{lccc} 
& B-value & $95 \%$ CI & p-value \\
\hline Age (yrs) & 0.38 & $0.01-0.02$ & $<0.001$ \\
Male gender & 0.20 & $0.09-0.19$ & $<0.001$ \\
Smoking & 0.09 & $0.01-0.13$ & 0.02 \\
Diabetes Mellitus & 0.10 & $0.03-0.22$ & 0.01 \\
Family history & 0.03 & $-0.03-0.07$ & 0.44 \\
Systolic blood pressure (mm Hg) & 0.23 & $0.00-0.01$ & $<0.001$ \\
BMI $\left(\mathrm{kg} / \mathrm{m}^{2}\right.$ ) & 0.03 & $-0.00-0.01$ & 0.39 \\
Total cholesterol (mmol/L) & 0.01 & $-0.02-0.03$ & 0.85 \\
Typical chest pain & -0.00 & $-0.08-0.08$ & 0.97 \\
Lg10 CCL3 & 0.04 & $-0.03-0.10$ & 0.25 \\
Lg10 CCL5 & -0.03 & $-0.04-0.02$ & 0.46 \\
Lg10 CCL18 & 0.10 & $0.03-0.22$ & 0.007 \\
\hline
\end{tabular}

Univariable linear regression model. $\mathrm{BMI}=$ body mass index, $\mathrm{CCL}=\mathrm{CC}$ chemokine ligand.

Table 4. Multivariable logistic regression analysis to predict for CAD ( $\geq 50 \%$ stenosis)

\begin{tabular}{lccc} 
& OR & $95 \%$ CI & p-value \\
\hline Model 1 & & & \\
Lg10 CCL3 & 1.01 & $0.67-1.53$ & 0.96 \\
Lg10 CCL5 & 1.23 & $0.99-1.53$ & 0.06 \\
Lg10 CCL18 & 1.03 & $0.55-1.93$ & 0.92 \\
Model 2 & & & \\
Lg10 CCL3 & & & \\
Lg10 CCL5 & 0.96 & $0.62-1.47$ & 0.84 \\
Lg10 CCL18 & 1.27 & $1.02-1.59$ & 0.04 \\
\hline
\end{tabular}

Model 1: corrected for age and gender, model 2: corrected for age, gender, diabetes mellitus, BMI, smoking, family history, systolic blood pressure and total cholesterol. CAD=coronary artery disease, $\mathrm{CCL}=\mathrm{CC}$ chemokine ligand, $\mathrm{OR}=$ odds ratio, $\mathrm{CI}=$ confidence interval.

\section{CCL concentrations and events}

During a mean 26 months follow-up, a total of 51 events occurred ( 3 all-cause mortality, 13 ACS, 10 CABG and 25 PCI). Patients who developed an event during follow-up were more often males, smokers and had higher calcium and segment involvement scores. Also, their median CCL5 concentration was significantly higher (table 5). When corrected for traditional risk factors in multivariable regression analysis, CCL5 concentration showed independent predictive value for the occurrence of an event during follow-up (table 6, model 2). When also corrected for the extent of CAD (adding segment involvement score, model 6), the independent prognostic value of CCL5 remained present. 
However, when correcting for the presence of significant stenosis $(\geq 50 \%$ stenosis, model 5), the significant prognostic value of CCL5 disappeared.

Table 5. Difference between patients developing an events and patients that do not develop an event

$$
\text { Patients without event Patients with event p-value }
$$

$$
n=661
$$

\begin{tabular}{lccc} 
& & $\mathrm{n}=51$ & 0.29 \\
Age (yrs) & $56 \pm 10$ & $58 \pm 11$ & 0.01 \\
Male gender & $361(54.6 \%)$ & $37(72.5 \%)$ & 0.002 \\
Smoking & $147(22.2 \%)$ & $22(43.1 \%)$ & 0.29 \\
Diabetes Mellitus & $52(7.9 \%)$ & $6(11.8)$ & 0.88 \\
Family history & $252(38.1 \%)$ & $20(39.2 \%)$ & 0.67 \\
Systolic blood pressure (mm Hg) & $142 \pm 19$ & $143 \pm 20$ & 0.81 \\
BMI $\left(\mathrm{kg} / \mathrm{m}^{2}\right)$ & $27 \pm 4$ & $27 \pm 4$ & 0.61 \\
Total cholesterol (mmol/L) & $5.4 \pm 1.1$ & $5.3 \pm 1.3$ & 0.67 \\
Typical chest pain & $81(12.3 \%)$ & $7(13.7 \%)$ & $<0.001$ \\
Calcium score & $4.6(0.0-103.1)$ & $181.0(20.6-458.2)$ & $<0.001$ \\
Involvement score & $1.0(0.0-3.0)$ & $5.0(2.0-7.0)$ & 0.85 \\
Radiation dose $(\mathrm{mSv})$ & $4.0(3.0-5.8)$ & $4.1(3.0-5.8)$ & 0.96 \\
CCL3 (pg/mL) & $2.7(0.0-7.0)$ & $2.7(0.0-6.7)$ & 0.005 \\
CCL5 (pg/mL *102) & $34.4(15.0-79.6)$ & $55.6(31.2-115.6)$ & 0.23 \\
CCL18 (pg/mL *102) & $769(456-1266)$ & $941(530-1272)$ & \\
\hline
\end{tabular}

Values are presented as numbers (\%), means \pm standard deviation or medians (IQR). Significant pvalues are in bold. $\mathrm{BMI}=$ body mass index, $\mathrm{CCL}=\mathrm{CC}$ chemokine ligand. 
Table 6. Multivariable logistic regression analysis to predict for event

\begin{tabular}{lccc} 
& OR & $95 \%$ CI & p-value \\
\hline Model 1 & & & \\
Lg10 CCL3 & 0.81 & $0.41-1.60$ & 0.54 \\
Lg10 CCL5 & 1.71 & $1.07-2.73$ & 0.03 \\
Lg10 CCL18 & 1.33 & $0.45-3.98$ & 0.61 \\
Model 2 & & & \\
Lg10 CCL3 & 0.88 & $0.43-1.78$ & 0.71 \\
Lg10 CCL5 & 1.60 & $1.01-2.52$ & 0.046 \\
Lg10 CCL18 & 1.35 & $0.45-4.10$ & 0.59 \\
Model 3 (CT 250\%) & & & \\
Lg10 CCL3 & 0.96 & $0.44-2.07$ & 0.91 \\
Lg10 CCL5 & 1.35 & $0.88-2.06$ & 0.17 \\
Lg10 CCL18 & 1.46 & $0.45-4.72$ & 0.53 \\
Model 4 (involvement score) & & & \\
Lg10 CCL3 & & & \\
Lg10 CCL5 & 0.75 & $0.35-1.59$ & 0.45 \\
Lg10 CCL18 & 1.62 & $1.03-2.57$ & 0.04 \\
\hline
\end{tabular}

Model 1: corrected for age and gender, model 2: corrected for age, gender, diabetes, BMI, smoking, family history, systolic blood pressure and total cholesterol, model 3: corrected for age, gender, diabetes, BMI, smoking, family history, systolic blood pressure, total cholesterol and $\geq 50 \%$ stenosis on CCTA. Model 4: corrected for age, gender, diabetes, BMI, smoking, family history, systolic blood pressure, total cholesterol and CCTA involvement score. $\mathrm{CAD}=$ coronary artery disease, $\mathrm{CCL}=\mathrm{CC}$ chemokine ligand, $\mathrm{OR}=$ odds ratio, $\mathrm{CI}=$ confidence interval.

\section{Additive predictive value of CCL5}

We evaluated the additional value of CCL5 on top of traditional risk factors by calculating two ROC models (figure 3). To assess the value to predict for a $\geq 50 \%$ stenosis, a model incorporating clinical risk factors showed an AUC of 0.74 (95\% CI 0.70-0.78). When CCL5 was added to this model, the AUC was 0.75 (95\% CI 0.71-0.79), which was a non-significant increase $(\mathrm{p}=0.26)$. Further, the same models were calculated to predict for the occurrence of an event. The model incorporating only clinical risk factors showed an AUC of 0.67 (95\% CI 0.60-0.75). When CCL5 was added to the model, the AUC increased to $0.68(95 \%$ CI $0.61-0.76), \mathrm{p}=0.38$. 
Figure 3. ROC analysis exploring additive value of CCL5 to predict for obstructive CAD and cardiac events
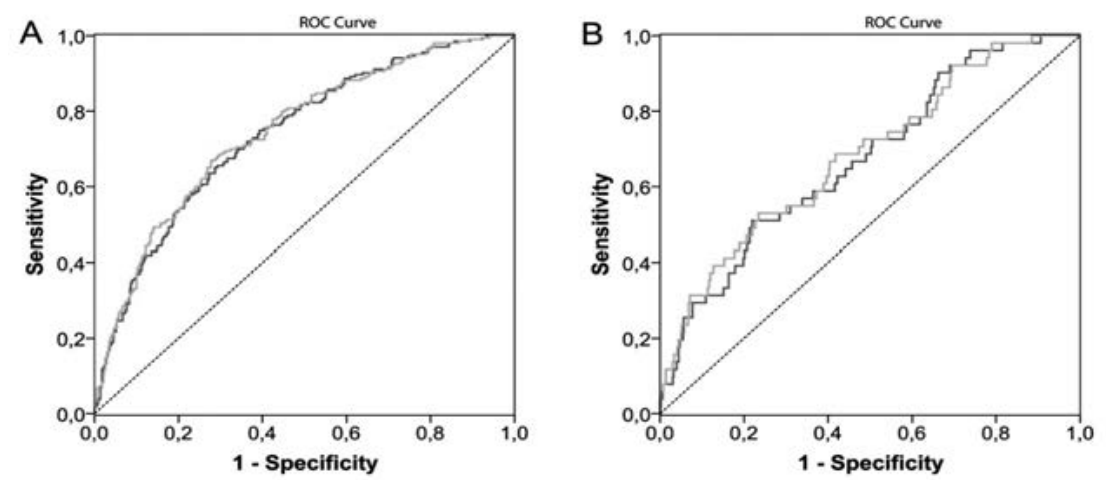

Panel A shows a ROC model of clinical risk factors to predict for the presence of one or more $\geq 50 \%$ stenoses, which obtained an AUC of 0.74 (95\% confidence interval $0.70-0.78$ ). When CCL5 was added to the model, the AUC did not change significantly 10.75 ; $95 \%$ confidence interval $0.71-0.79$ ), $\mathrm{p}=0.26$.

Panel B shows the same models predicting for the occurrence of a cardiac event. The AUC for clinical risk factors was 0.67 (95\% confidence interval $0.60-0.75)$, which was essentially unaltered (0.68; (95\% confidence interval $0.61-0.76)$ when CCL5 was added to the model, $\mathrm{p}=0.38$.

\section{Exploration on CCL levels and timing ACS}

We further explored the relation between time of ACS and the concentration of CCL3, 5 and 18. Therefore, an 'early ACS' ( $\mathrm{n}=7$ ) and 'late ACS' group ( $\mathrm{n}=6$ ) was created, by dividing the ACS group into two equal groups (using median time between CCTA and ACS [134 days] as cutoff). Interestingly, the concentration of CCL5 was markedly elevated in patients that developed ACS soon, as compared to patients with late or no ACS (figure 4). Such a relation between time of ACS and concentration was not found for CCL3 or CCL18.

In addition, also when CCL5 concentration was defined as low or high (using median as cutoff; $8,514 \mathrm{pg} / \mathrm{mL}$ ) within the ACS group ( $\mathrm{n}=13$ ), the median time to event for low CCL5 concentrations was; 469 days (149-675), as compared to 41 days (41-134) for high CCL5, $\mathrm{p}=0.007$. 
Figure 4. Difference in median CCL3, 5 and 18 concentration between no, early and late ACS
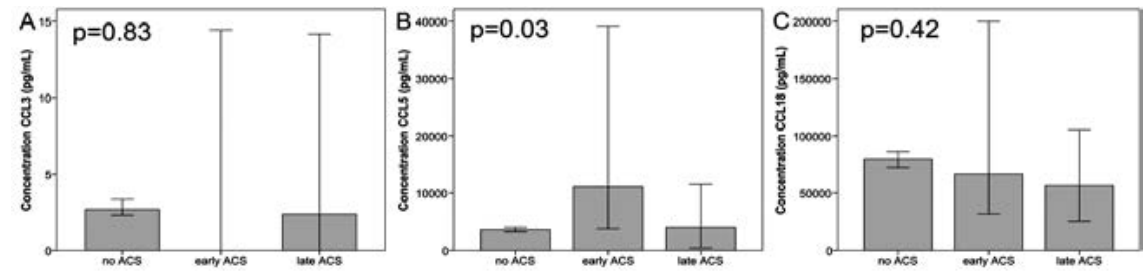

CCL5 concentrations were significantly higher in patients who developed an ACS relatively early after CCTA ( $\leq 134$ days) only. For late ACS, CCL5 concentration was similar to patients that did not develop an event (panel B). Such a relation was not found for CCL3, nor for CCL18 (panel A, C).

\section{DISCUSSION}

In the present study, we investigated the association of CCL3, 5 and 18 with the severity and extent of CAD, as well as their predictive value for the occurrence of primary cardiac events. The main finding is that CCL5 demonstrated independent value to predict for the presence of significant CAD. CCL18 was significantly associated with calcium and involvement scores. In addition, CCL5 but not CCL3 and CCL18 provided prognostic value for the occurrence of an event during follow-up, independent from both traditional clinical risk factors as well as extent of CAD.

Previously, CCL3, 5 and 18 serum levels have been linked to cardiovascular disease (secondary events in early follow-up, refractory UAP, and ACS) and have been shown to be expressed by atherosclerotic plaque. Also, CCL5 has been related to carotid plaque characteristics. ${ }^{18}$ However, to our knowledge, the association of CCL3, 5 and 18 with CAD in a stable, outpatient population as well as the prognostic value for primary ACS has not yet been investigated. Previous studies on CCL3, 5 and 18 have focused on the post-ACS stage, pointing to divergent source and roles with regard to cardiovascular disease. For instance, CCL5 and CCL18 have shown to be transiently elevated during ischemia. Especially CCL5 has been strongly associated with platelet activation, and serum levels are altered only transiently in the acute phase of unstable angina pectoris. ${ }^{6}$ This is in line with our hypothesis generating finding that CCL5 concentrations were significantly higher in patients developing ACS relatively early after CCTA ( $\leq 134$ days), as compared to late ACS cases. In our study, CCL5 was independently associated with the presence of $\geq 50 \%$ coronary stenosis, a well-known risk factor for the occurrence of a cardiac event. ${ }^{19,}{ }^{20}$ In 
fact, CCL5 serum levels also correlated with the occurrence of cardiac events. This association was independent from traditional risk factors. This independent association remained even when including the extent of CAD, which is a known risk factor. However, when a $\geq 50 \%$ coronary stenosis was added to the model, the significant association disappeared. Apparently, CCL5 is elevated in case of coronary obstruction, and not in case of extensive CAD only. Possibly, CCL 5 serum levels are reflective of platelet activation status ${ }^{6}$ which could become manifest in obstructive CAD only. Therefore, CCL5 might be promising as an additional risk factor candidate, before cardiac imaging diagnostics are applied. However, when we calculated ROC models, we could not objectify a significant incremental value of CCL5, neither for the prediction of significant CAD, nor for cardiac events. Consequently, the value of CCL5 as ACS prognosticator in this study is limited. However, apart from a risk factor, CCL5 might have potential as a therapeutic target. Due to its strong association with CAD and events, pharmacologic inhibition of CCL5 could have potential to reduce plaque formation. Moreover, previous research showed that $\mathrm{CC}$ chemokine receptor (CCR) 5 deficiency protected men for (early) myocardial infarction ${ }^{21}$, and mice for early plaque formation. ${ }^{22}$

On the other hand, CCL18 showed a particular association with coronary calcium score, as well as segment involvement score. This might be explained by the fact that CCL18 can directly stimulate fibrosis, ${ }^{4,23}$ which might effect in calcification and more extensive plaque formation. Although CCL18 was not associated with events, it might be associated to the underlying mechanisms such as atherosclerosis progression. CCL18 showed a clear univariable association with extent of CAD as measured by coronary calcium score and involvement score. Such an association was not found with obstructive CAD, although there was also a trend towards significance for higher CCL18 concentrations in patients with a $\geq 50 \%$ coronary stenosis. However, in multivariable analysis, CCL18 was neither independently associated to CAD (not for calcium, segment involvement score or obstructive CAD), nor to occurrence of events. This is in contrast to other prospective studies, in which CCL18 appeared to be an independent predictor of secondary cardiovascular events in the immediate aftermath of AMI or UAP. ${ }^{8}{ }^{24}$ In a stable chest pain population however, CCL18 seems to be calcification and plaque mass dependent, but not predictive for future events. We have to bear in mind that blood serum measurements of CCL18 might not be reflecting CCL activity within atherosclerotic plaque for- 
mation, as a study investigating carotid plaques found an increased expression of CCL18, which was not reflected in increased serum levels. ${ }^{9}$

The fact that we did not find an association between CCL3 and CAD and/or events, might seem contradictive to previous publications. For instance, higher CCL3 (and CCL5) concentrations were identified in patients with acute myocardial infarction as compared with controls. ${ }^{7}$ Also, CCL3 (as well as CCL5, 18) was independently associated with short-term mortality during follow-up in ACS patients. ${ }^{8}$ Importantly, we measured CCL3, 5 and 18 concentrations prior to events. Therefore, collectively the data on CCL3 seem to plead for a role of CCL3 in the acute phase of an ischemic event, not in the etiology of ACS. CCL3 seems predominantly ischemia derived ${ }^{7}$ and may be involved in post ischemia inflammation, possibly through neutrophil induced recruitment of monocytes. ${ }^{25}$

In summary, we report novel information on the role of CCL3, 5 and 18 in stable chest pain patients. CCL5 demonstrates an independent association with the presence of CAD as well as with the occurrence of cardiac events, while CCL18 levels were seen to correlate with cumulative plaque burden and calcification. In this population however, none of the CCL markers studied represent a clear incremental value for primary ACS risk prediction.

\section{Limitations}

We studied a relatively large population of patients with chest discomfort, who underwent CCTA to rule out obstructive CAD. Although this population meets our objectives, with the current cohort size, the low event rate of this low risk population might have limited the value of longitudinal analyses. Also, this may cloud significances after adjustment for confounding risk factors and precludes attempts to categorize the event group. Therefore, our attempts to further explore relations between ACS timing and CCL levels can at best be regarded hypotheses generating. Revascularization need may be biased by the baseline CCTA. Finally, blood was drawn only at baseline not during follow-up, which precludes assessment of chemokine pattern dynamics prior to event.

\section{Conclusions}

In stable chest pain patients, CCL5 and 18 were associated with coronary obstruction and extent of CAD including calcification, respectively. These data suggest relevance of CCL5 and 18 for CAD development, and identify them as 
potential pharmacological targets for CAD treatment. Furthermore, CCL5 appeared to be associated with the occurrence of future cardiac events. This novel information implicates that CCL5 could be a useful marker to predict for CAD and its consequences.

\section{References}

1. Hansson GK, Hermansson A. The immune system in atherosclerosis. Nat Immunol 2011; 12(3):204-12.

2. Charo IF, Taubman MB. Chemokines in the pathogenesis of vascular disease. Circ Res 2004;95(9):858-66.

3. Weber C. Platelets and chemokines in atherosclerosis: partners in crime. Circ Res 2005;96(6):612-6.

4. Weber C, Schober A, Zernecke A. Chemokines: key regulators of mononuclear cell recruitment in atherosclerotic vascular disease. Arterioscler Thromb Vasc Biol 2004;24(11): 1997-2008.

5. Reape TJ, Rayner K, Manning CD, Gee AN, Barnette MS, Burnand KG, et al. Expression and cellular localization of the CC chemokines PARC and ELC in human atherosclerotic plaques. Am J Pathol 1999;154(2):365-74.

6. Kraaijeveld AO, de Jager SC, de Jager WJ, Prakken BJ, McColl SR, Haspels I, et al. CC chemokine ligand-5 (CCL5/RANTES) and CC chemokine ligand-18 (CCL18/PARC) are specific markers of refractory unstable angina pectoris and are transiently raised during severe ischemic symptoms. Circulation 2007;116(17):1931-41.

7. de Jager SC, Kraaijeveld AO, Grauss RW, de Jager W, Liem SS, van der Hoeven BL, et al. CCL3 (MIP-1 alpha) levels are elevated during acute coronary syndromes and show strong prognostic power for future ischemic events. J Mol Cell Cardiol 2008;45(3):446-52.

8. de Jager SC, Bongaerts BW, Weber M, Kraaijeveld AO, Rousch M, Dimmeler S, et al. Chemokines CCL3/MIP1alpha, CCL5/RANTES and CCL18/PARC are independent risk predictors of short-term mortality in patients with acute coronary syndromes. PLoS One 2012;7(9):e45804.

9. Hagg DA, Olson FJ, Kjelldahl J, Jernas M, Thelle DS, Carlsson LM, et al. Expression of chemokine (C-C motif) ligand 18 in human macrophages and atherosclerotic plaques. Atherosclerosis 2009;204(2):e15-20.

10. Versteylen MO, Joosen IA, Shaw LJ, Narula J, Hofstra L. Comparison of Framingham, PROCAM, SCORE, and Diamond Forrester to predict coronary atherosclerosis and cardiovascular events. J Nucl Cardiol 2011;18(5):904-11. 
11. Shrimpton P. Assessment of patient dose in CT: appendix C-European guidelines for multislice computed tomography. European Commission project MSCT: CT safety \& efficacy—a broad perspective. http://www.msct.eu/PDF_FILES/Appendix $\% 20$ paediatric\%20CT\%20Dosimetry.pdf. Published 2004. Accessed November 20, 2008.

12. Agatston AS, Janowitz WR, Hildner FJ, Zusmer NR, Viamonte M, Jr., Detrano R. Quantification of coronary artery calcium using ultrafast computed tomography. J Am Coll Cardiol 1990;15(4):827-32.

13. Austen WG, Edwards JE, Frye RL, Gensini GG, Gott VL, Griffith LS, et al. A reporting system on patients evaluated for coronary artery disease. Report of the Ad Hoc Committee for Grading of Coronary Artery Disease, Council on Cardiovascular Surgery, American Heart Association. Circulation 1975;51(4 Suppl):5-40.

14. Min JK, Shaw LJ, Devereux RB, Okin PM, Weinsaft JW, Russo DJ, et al. Prognostic value of multidetector coronary computed tomographic angiography for prediction of all-cause mortality. J Am Coll Cardiol 2007;50(12):1161-70.

15. Friedewald WT, Levy RI, Fredrickson DS. Estimation of the concentration of low-density lipoprotein cholesterol in plasma, without use of the preparative ultracentrifuge. Clin Chem 1972;18(6):499-502.

16. Braunwald E, Antman EM, Beasley JW, Califf RM, Cheitlin MD, Hochman JS, et al. ACC/AHA 2002 guideline update for the management of patients with unstable angina and non-ST-segment elevation myocardial infarction--summary article: a report of the American College of Cardiology/American Heart Association task force on practice guidelines (Committee on the Management of Patients With Unstable Angina). J Am Coll Cardiol 2002;40(7):1366-74.

17. DeLong ER, DeLong DM, Clarke-Pearson DL. Comparing the areas under two or more correlated receiver operating characteristic curves: a nonparametric approach. Biometrics 1988;44(3):837-45.

18. Virani SS, Nambi V, Hoogeveen R, Wasserman BA, Coresh J, Gonzalez F, 2nd, et al. Relationship between circulating levels of RANTES (regulated on activation, normal T-cell expressed, and secreted) and carotid plaque characteristics: the Atherosclerosis Risk in Communities (ARIC) Carotid MRI Study. Eur Heart J 2011;32(4):459-68.

19. Min JK, Dunning A, Lin FY, Achenbach S, Al-Mallah M, Budoff MJ, et al. Age- and sexrelated differences in all-cause mortality risk based on coronary computed tomography angiography findings results from the International Multicenter CONFIRM (Coronary CT Angiography Evaluation for Clinical Outcomes: An International Multicenter Registry) of 23,854 patients without known coronary artery disease. J Am Coll Cardiol 2011;58(8):84960.

20. Versteylen MO, Joosen IA, Winkens MH, Laufer EM, Snijder RJ, Wildberger JE, et al. Combined use of exercise electrocardiography, coronary calcium score and cardiac CT angiography for the prediction of major cardiovascular events in patients presenting with stable chest pain. Int J Cardiol 2012. 
21. Gonzalez P, Alvarez R, Batalla A, Reguero JR, Alvarez V, Astudillo A, et al. Genetic variation at the chemokine receptors CCR5/CCR2 in myocardial infarction. Genes Immun 2001;2(4):191-5.

22. Potteaux S, Combadiere C, Esposito B, Lecureuil C, Ait-Oufella H, Merval R, et al. Role of bone marrow-derived CC-chemokine receptor 5 in the development of atherosclerosis of low-density lipoprotein receptor knockout mice. Arterioscler Thromb Vasc Biol 2006;26(8):1858-63.

23. Atamas SP, Luzina IG, Choi J, Tsymbalyuk N, Carbonetti NH, Singh IS, et al. Pulmonary and activation-regulated chemokine stimulates collagen production in lung fibroblasts. Am J Respir Cell Mol Biol 2003;29(6):743-9.

24. De Sutter J, Struyf S, Van de Veire NR, Philippe J, De Buyzere M, Van Damme J. Cardiovascular determinants and prognostic significance of CC Chemokine Ligand-18 (CCL18/PARC) in patients with stable coronary artery disease. J Mol Cell Cardiol;49(5):894-6.

25. Soehnlein O, Lindbom L, Weber C. Mechanisms underlying neutrophil-mediated monocyte recruitment. Blood 2009;114(21):4613-23. 



\section{Chapter}

\section{General discussion}




\section{Technical advances in cardiac computed tomographic angiography}

Cardiac imaging has become available due to the ongoing technical advances in diagnostic techniques. For many years, the only method capable of directly imaging the coronary arteries has been selective coronary angiography. Although computed tomography (CT) has long been a popular diagnostic modality to perform angiography, cardiac imaging has been problematic due to the mobile nature of the heart. With the introduction of 16- and later 64-slice CT, temporal resolution increased significantly, allowing the imaging of coronary arteries during diastase (the isovolumetric phase of the diastole). ${ }^{1-4}$ These faster CT scanners did enable visualization of the coronary artery tree of the moving heart, using intravascular contrast to enhance the coronary lumen and ECGgating to reconstruct still images. In contrast to conventional angiography, which basically is luminography, cardiac computed tomographic angiography (CCTA) is able to visualize all structures within the scan range. In addition to the contrast enhanced coronary lumen, it provides information about the vessel wall, the myocardium and remaining cardiac anatomy. The acquisition of CCTA is fast and non-invasive, which makes the technique easily available and applicable for clinical use.

\section{Radiation dose}

The greatest concern of employing CCTA has been the increased radiation dose it might deliver to the population. 5,6 Volumes in cardiac imaging diagnostics using ionizing radiation (including CCTA) have increased tremendously during the last decades. ${ }^{7}$ The detrimental effects of radiation have been described in atomic bomb survivors, in which people exposed to a mean $34 \mathrm{mSv}$ had a significantly higher cancer incidence. ${ }^{8}$ In addition, fetuses exposed to an estimated radiation doses of about $10 \mathrm{mSv}$ had increased risk for childhood cancer. ${ }^{9}$ Extrapolating these data to the low radiation dose accompanied by CCTA is complicated. The actual cancer risk implied by cardiac imaging is largely unknown and differs between gender and ages..$^{10}$ Because the effects are uncertain, we should be critically justifying all radiation exposure to our patients. 7 Therefore, attempts to lower the effective dose according to the ALARA (As Low As Reasonably Achievable) principle are essential in CCTA. ${ }^{11}$ Fortunately, with improvements in especially temporal resolution, CCTA radiation dose has declined substantially. First, the development of prospectively ECG-triggered scanning protocols and ECG-triggered dose modulation have 
resulted in a vast decline in radiation dose. ${ }^{12,} 13$ Also the introduction of dualsource CT scanners enabled CCTA acquisition at a very low dose. ${ }^{14,15}$ Another state-of-the-art development is the introduction of iterative reconstruction. Using this reconstruction technique, image noise can be reduced, which enables a similar image quality using lower radiation dose. ${ }^{16-18}$ It is therefore expected that radiation dose of CCTA will decrease even further in the coming years to a range from 1 to $5 \mathrm{mSv}$, depending on scan protocol. ${ }^{12,14}$ This is a great improvement compared to mean radiation doses of $10-20 \mathrm{mSv}$ for CCTA that were reported in early studies. ${ }^{19,} 20$ In addition, the radiation dose that accompanies other diagnostic techniques is often higher. For instance, conventional angiography exposes patients to 5-7 mSv. ${ }^{18,} 19,21 \mathrm{Also}, 10-20 \mathrm{mSv}$ has been reported for most nuclear imaging techniques ${ }^{22}$, although it is fair to say that due to similar advances, radiation dose is decreasing significantly as well for myocardial perfusion imaging, to about 6-9 mSv. ${ }^{23}$

\section{Diagnosing coronary artery disease with CCTA}

\section{Eligible patients}

Patient selection is an important part of every diagnostic test. When diagnosing coronary artery disease (CAD), the theory of conditional probability (or Bayes' theorem) is a crucial aspect. ${ }^{24}$ In short, the risk of having CAD after a diagnostic test, depends on the pre-test risk for CAD. Therefore, the additional value of a test on top of pre-test probability varies. Consequently, assessing the use of diagnostic testing on an individual basis is crucial. Comparisons between CCTA and conventional angiography have shown that CCTA has a specifically high negative predictive value. ${ }^{25},{ }^{26}$ Consequently, CCTA has been considered to be a suitable test to 'rule-out' CAD. Therefore, CCTA is considered an appropriate diagnostic test in low- to intermediate risk patients. ${ }^{27} \mathrm{~A}$ negative test result in this group of patients can effectively rule out CAD. Further, diagnostic value can be impaired by factors such as age, obesity and atrial fibrillation. ${ }^{28}$ As these factors will require higher radiation dose and more downstream testing, referent physicians should consider whether the choice to perform CCTA is justified in every individual patient.

\section{Diagnostic value of CCTA}

The diagnostic accuracy of CCTA, as compared to the gold standard conventional coronary angiography, is well established. Especially sensitivity and nega- 
tive predictive value of the technique are high. ${ }^{26,}{ }^{29}$ Specificity and positive predictive value are less established, and early studies reported values ranging from 65 to $90 \%$ and from 13 to $91 \%$, respectively. $25,26,29,30$ A particular limitation of CCTA is the evaluation of highly calcified CAD, predominantly due to socalled 'blooming' artifacts, or partial volume artifacts of calcified plaque material..$^{31}$ These complicate the evaluation of luminal stenoses, often leading to follow-up diagnostics. Therefore, diagnostic accuracy is very dependent on patient selection. However, recent advances in technology have increased diagnostic accuracy. For instance, recent studies have shown that dual-source CT scanners using high pitch spiral acquisition mode increase the positive predictive power of CCTA. Several recent studies investigating high-pitch scanning have shown a specificity around 82 to $94 \%$ and a positive predictive value of 72 to $97 \% .32,33$ It can be expected that ongoing developments in both scanner hardware, acquisition and reconstruction techniques will enable further improvement of diagnostic ability.

\section{Prognostic implications of CCTA}

\section{The prognostic value of stenosis severity}

In addition to its diagnostic value, CCTA has important prognostic implications. Especially the presence of a $\geq 50 \%$ stenosis on CCTA has been identified as an important prognostic indicator. ${ }^{34,} 35$ Such a coronary obstruction is a strong risk factor for the occurrence of adverse events. The CONFIRM trial showed in almost 25,000 patients that obstructive CAD on CCTA independently predicted for the occurrence of all-cause mortality (risk-adjusted hazard ratio 2.6). ${ }^{36}$ Moreover, the presence of luminal stenosis as detected by CCTA seems to provide additive prognostic value over clinical risk profiling and even calcium scoring. ${ }^{37}$

Our own data show similar results. In chapter 5 we conclude that a $\geq 50 \%$ stenosis on CCTA has relatively more prognostic implications than a positive exercise ECG or a positive calcium score. Although exercise ECG provided some additional predictive value over CCTA, calcium score did not. This makes a standard calcium score scan performed with every CCTA debatable. The combination of CCTA with exercise ECG provided a very high prognostic yield. The results indicate that CCTA is a suitable test to obtain prognostic information in stable chest pain patients. Possibly, the addition of exercise ECG increases prognostic yield by compensating for the lack of functional 
information as provided by CCTA. Another approach to test functional significance is applying methoxyisobutylisonitrile-single photon emission computed tomography (MIBI-SPECT), which has a higher diagnostic accuracy as compared with exercise-ECG. ${ }^{38}$ Combining this with CCTA has enabled CCTASPECT imaging, which appreciates both anatomic as well as functional significance of coronary artery lesions. ${ }^{39}$

Although prognostic studies have focused on the presence of obstructive $\mathrm{CAD}$, multiple-vessel disease and other classic descriptions of CAD, CCTA is also very able to depict non-obstructive atherosclerosis. Presence of nonobstructive CAD independently predicted all-cause mortality in the CONFIRM trial (hazard ratio 1.6). ${ }^{36}$ Additionally, a meta-analysis among over 9,000 patients showed that with increasing severity of $\mathrm{CAD}$, the numbers of major adverse cardiovascular events gradually increased. 40 We show mildly different data, since patients without $\mathrm{CAD}$ had a higher than expected event rate, similar to patients with non-obstructive CAD. Patients harboring moderate and severe plaques showed a markedly higher event rate, figure 1 . As we describe in chapter 6, a normal CCTA cannot completely rule-out ACS in the future, especially in women, although the event rates are very low.

Figure 1. Survival according to CAD severity

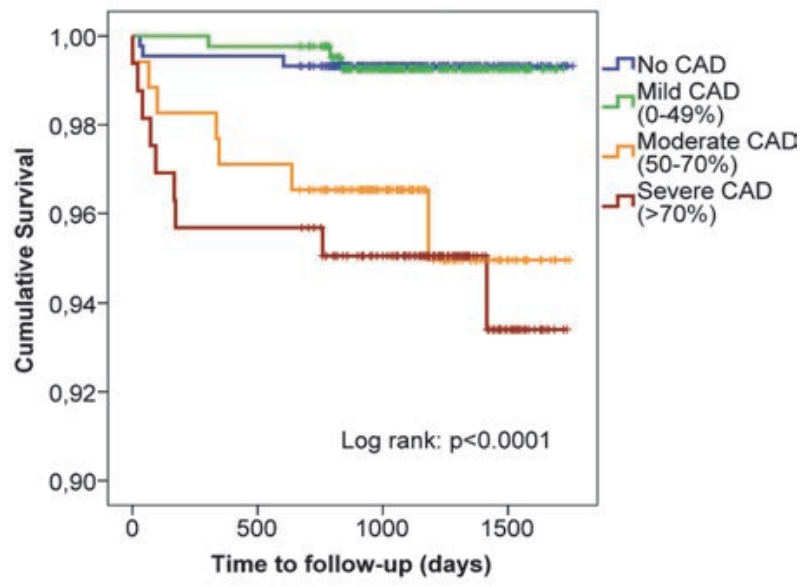

Kaplan-Meier curve for development of hard cardiovascular events (cardiac death, acute coronary syndrome incl. myocardial infarction) in patients with no CAD, mild CAD $(<50 \%)$, moderate CAD (50-70\%) and severe CAD (>70\% luminal stenosis). 


\section{Ability of CCTA to assess plaque characteristics}

From a conventional point of view, angiographic stenosis severity has been a hallmark in determining prognosis for the individual patient. Using CCTA however, imaging of the vessel wall provides additional information about plaque composition and geometry. Several studies have investigated the ability of CCTA to identify plaque characteristics, using intravascular ultrasound as the gold standard. ${ }^{41-44}$. The comparative studies between CCTA and IVUS have shown that partially calcified and calcified lesions can be detected quite accurately by CCTA, whereas non-calcified plaque volume is often underestimated. 43,45 Others reported that the differentiation between fibrous plaque content and lipid-rich plaque core using CCTA is difficult. ${ }^{41}$ Although CCTA might lack the spatial resolution to identify specific plaque characteristics such as fibrous cap thickness, there are several characteristics that have been investigated for prognostic value.

\section{Prognostic significance of CCTA derived plaque characteristics}

Multiple studies have demonstrated that CCTA derived plaque characteristics are associated with the occurrence of ACS.46-52 Several prognostic plaque characteristics have been described, especially presence of non-calcified and partially calcified plaques ${ }^{48,51}$, outward remodeling ${ }^{46}$ and low attenuation of plaques. ${ }^{47}$, 49, 50,52 Also, total plaque volume has been reported to be significantly higher in patients presenting with ACS. ${ }^{53}$ However, these studies all investigated patients who underwent CCTA at the time of their event. Although it is conceivable that phenotypes of plaques associated with coronary events characterize plaques that are vulnerable to rupture, these studies do not prove that noninvasive plaque imaging can actually predict for future events. Clinical evidence on coronary plaque characteristics as detected by CCTA subsequently resulting in ACS is very limited. ${ }^{44,55}$

In chapter 7 we report that detailed quantification of CAD by a dedicated semi-automated software algorithm beholds important prognostic value. Not only did this enable us to identify plaque characteristics associated with future ACS, but utilizing the algorithm also provided incremental prognostic value over clinical risk profiling and conventional CCTA reading. This information is of clinical significance since it implicates that we can obtain additional prognostic information from available imaging datasets, without additional costs or radiation dose. Some limitations are that the analysis is still time-consuming and subject to inter-observer variability. Therefore, the developments of automated 
quantification algorithms are very promising for more general clinical use. Amongst the most important global predictors for subsequent ACS were: total non-calcified plaque volume, the total volume of all plaque and the total number of plaques in the whole coronary artery tree. The most predictive local parameters were highest volume and highest plaque burden of any plaque present. In a study investigating post- $A C S$ patients, non calcified plaque volume as well as total plaque volume were independent predictors for future cardiac events. ${ }^{56}$ In addition to global plaque measures, we also showed predictive value of plaques with the highest area, the highest non-calcified percentage, the lowest attenuation, and the highest remodeling index. In a recent study, several plaque characteristics were investigated in a population undergoing IVUS after their first ACS. ${ }^{57}$ After follow-up, a high plaque burden and a low minimal luminal area were among the strongest predictors of a subsequent cardiovascular event in this study. These results in a high risk population, using another diagnostic modality, are still in part in line with our findings. What these studies have in common, is that they underline the prognostic feasibility of volumetric plaque burden and geometric plaque characteristics. In my opinion, these characteristics may provide more prognostic power than stenosis severity, and it seems logical to pursue further studies into this direction.

\section{Can CCTA identify high-risk patients?}

The definition and detection of 'vulnerable' plaques remain to be difficult. The fact that multiple pathophysiological mechanisms have been proposed for

plaque vulnerability make it an elusive phenomenon. ${ }^{58}$ The most common cause of acute coronary syndrome (ACS) is plaque rupture resulting in intraluminal thrombosis..$^{59}$ It occurs probably frequently, and often subclinically. ${ }^{60} \mathrm{In}$ about $70 \%$ of cases, these are rupture prone thin-capped fibroatheromas. These plaques typically harbor a large lipid-rich atheromatous core, characterized on CCTA by a large volume and low-attenuation. Although low-attenuation plaque core is detectable by CCTA ${ }^{61}$, attenuation values are subject to contrast concentration, tube voltage and kernel used at the time of image acquisition. ${ }^{62-64}$ This complicates the detection of lipid-rich plaque cores. Another approach is to investigate structural pattern of plaque. Maurovich-Horvat et al. showed that the specific patterns correlated well with thin cap fibroatheromas. ${ }^{65}$ Nonetheless, although exact volumetric measurement of low-attenuation plaque may be arbitrary, plaque attenuation has shown to behold prognostic value. ${ }^{54,} 55$ In chapter 7 of this thesis, we show that the presence of high plaque volumes and 
low attenuation indeed predicted for the occurrence of ACS. Several invasive techniques are available that enable detailed plaque characteristic identification, such as IVUS and OCT. However, there are several advantages of using CCTA. First, the technique is non-invasive. In contrast to IVUS/OCT in which usually a selected number of coronary segments are imaged, CCTA enables the visualization of all coronary segments. This enables the technique to quantify atherosclerotic burden in the entire coronary tree, acknowledging the diffuse nature of atherosclerotic disease. As we have shown, such an approach can have important prognostic implications.

Another frequent cause of ACS is plaque erosion 66,67 , which occurs more often in younger women. ${ }^{68}$ Loss or dysfunction of luminal endothelial cells lining these plaques can provoke intraluminal thrombosis. In chapter 6 we show that in women, ACS more frequently occurs in the presence of nonobstructive lesions as detected by CCTA. It is therefore conceivable that the process of plaque erosion might play a mechanistic role in these events. We even report events in patients with a previously normal CCTA. This makes the prospective identification of plaques vulnerable to plaque erosion particularly difficult. The use of non-invasive imaging alone might be insufficient in detecting such vulnerable plaques.

The less prevalent vulnerable plaque type is the calcified nodule.59, 69 Often, fibrin is present along with inflammatory cells. The clinical significance of these plaques is not well understood. Although calcium can be easily detected on CCTA, the differentiation between vulnerable and normal calcified plaques will be very problematic. There is evidence that the presence of non calcified plaque volume is a more potent predictor for cardiac events than calcium score or calcified volume. ${ }^{56,70}$ None of the cardiac events that we described in our studies occurred in patients with solely calcified lesions. This information in combination with the low prevalence make this type of vulnerable plaque clinically less relevant.

As we have shown, it might be more feasible to investigate the presence of high risk atherosclerosis throughout the coronary artery tree as opposed to the focus on a single vulnerable plaque. The identification of high risk CAD contributes a great deal to the identification of high-risk patients. Although coronary plaques play a significant role, the formation of intracoronary thrombus is multifactorial. Pro-thrombotic status of the blood and fibrous cap inflammation are among the most important, but also variable factors. ${ }^{71}$ Unfortunately, these parameters are not addressed with imaging. For instance, inflam- 
matory and thrombotic biomarkers could potentially aid the identification of the vulnerable patient. In research projects out of the scope of this dissertation, we have detected the association between extent of CAD and in vivo thrombin formation, ${ }^{72}$ as well as troponin $T .{ }^{73}$ Troponin even has potential to add prognostic value on top of CCTA. ${ }^{74}$ This is in contrast to the inflammatory marker hs-CRP, which did not show prognostic value in this study.

\section{Does CCTA provide a 'warranty period'?}

Although previous research suggested that a normal CCTA provides an up to 7 year event free 'warranty period' ${ }^{75}$, there is no such data yet for patients with low-risk plaque characteristics. In our studies, clinical follow-up was relatively short. For instance, mean follow-up was approximately 2.5 years. Although the prognostic value of plaque characteristics are very promising, it is unclear whether they can predict for events on the long term. It is conceivable that high risk CAD is likely to result in ACS in a relative short time window. To further investigate this, we separated the group of patients who developed ACS in two equal groups, an 'early event group' and 'late event group'. Interestingly, the value of several plaque characteristics to predict for early events seemed higher as compared with the prediction of late events (Table 1). In the late event group no significant differences were noted, while in the early event group ACS patients had a significantly higher non-calcified volume, and there was a trend towards significance for higher plaque burden and remodeling index. Although these groups are small and results underpowered, this could have implications for the clinical use of CCTA plaque quantification. 
Table 1. The influence of time-to-event on difference in semi-automatically quantified plaque characteristics between culprit lesions and non-culprit plaques within patients who developed ACS $(n=24)$.

\begin{tabular}{lccc} 
& Culprit plaques & Non-culprit plaques & P-value \\
\hline All events & $\mathrm{n}=24$ & $\mathrm{n}=74$ & 0.04 \\
Plaque total volume (mm3) & $25.4(7.3-69.6)$ & $9.5(1.9-43.8)$ & 0.005 \\
Plaque non-calcified volume (mm3) & $3.4(0-18.8)$ & $0(0-5.4)$ & 0.02 \\
Plaque burden (\%) & $39.0(24.5-56.0)$ & $29.0(12.0-39.3)$ & 0.04 \\
Plaque area (mm2) & $5.0(2.4-8.6)$ & $3.3(0.9-5.9)$ & 0.04 \\
Plaque attenuation (HU) & $287(195-543)$ & $468(323-557)$ & 0.06 \\
Plaque remodeling index & $1.3(1.2-1.5)$ & $1.2(1.0-1.4)$ & $\mathrm{n}=46$ \\
Early event (within 165 days FU) & $\mathrm{n}=11$ & $8.4(1.5-45.0)$ & 0.13 \\
Plaque total volume (mm3) & $22.8(7.9-44.8)$ & $0(0-1.0)$ & 0.03 \\
Plaque non-calcified volume (mm3) & $0.9(0-19.2)$ & $27.0(11.0-37.5)$ & 0.08 \\
Plaque burden (\%) & $38.0(27.0-57.0)$ & $2.8(0.9-6.3)$ & 0.12 \\
Plaque area (mm2) & $5.2(2.4-7.1)$ & $492(327-660)$ & 0.22 \\
Plaque attenuation (HU) & $374(234-589)$ & $1.2(1.0-1.5)$ & 0.05 \\
Plaque remodeling index & $1.5(1.3-1.6)$ & $\mathrm{n}=28$ & 0.13 \\
Late event (after 165 days FU) & $\mathrm{n}=13$ & $10.1(2.9-31.1)$ & 0.18 \\
Plaque total volume (mm3) & $27.9(6.4-96.0)$ & $0(0-8.1)$ & 0.15 \\
Plaque non-calcified volume (mm3) & $3.4(0.4-22.8)$ & $30.0(19.0-41.5)$ & 0.14 \\
Plaque burden (\%) & $40.0(23.0-57.0)$ & $399(317-488)$ & 0.16 \\
Plaque area (mm2) & $3.9(2.2-8.9)$ & & 0.45 \\
Plaque attenuation (HU) & $284(124-532)$ & $(1.1-1.4)$ & \\
Plaque remodeling index & $1.3(1.2-1.4)$ & & \\
\hline
\end{tabular}

Variables are described as median (interquartile range), p-values are calculated using MannWhitney test. ACS=acute coronary syndrome, HU=Hounsfield unit, FU=follow-up.

\section{Chest pain in the era of CCTA}

Chest pain symptoms can have numerous origins, of which myocardial ischemia is just one. Esophageal reflux, irritated thoracic muscles, ribs, diaphragm, lungs, are just an example of possible causes of thoracic pain. Initial evaluation consisting of thoroughly taken history and physical examination will differentiate most non-cardiac from possible cardiac causes. For clinical cardiologists, assessment of patients with stable chest pain of possible cardiac origin remains a challenge. As we have seen in chapter 2 , the performance of traditional clinical risk factors in predicting CAD is only moderate. Already decades ago, a large number of these patients showed normal coronary arteries on conventional angiography. ${ }^{76,77}$ It has often been stated that such patients could harbor non-obstructive CAD which is not visible by conventional angiography, but can still cause complaints through mechanisms of spasm or endothelial dys- 
function. Moreover, an apparently large amount of patients with normal conventional angiography studies show atherosclerosis on IVUS. ${ }^{78,79}$ Now that CCTA has become available, we still observe a large amount of patients with chest pain despite normal coronary arteries. Coronary spasm and endothelial dysfunction cannot be ruled out with CCTA. Also, the technique is only capable of visualizing epicardial vessels. As described in chapter 6, the prognosis of women with typical chest pain and normal coronaries is not as benign as one could expect. This is in line with previous publications, in which the risk for ACS was higher in patients with endothelial dysfunction despite normal coronary arteries, especially in women. ${ }^{80,81}$ So besides obscure atherosclerosis, disturbances in endothelial function can be a mechanism resulting in ischemia in these patients. ${ }^{82}$ We therefore proposed that additional diagnostic testing could be useful in risk stratifying this group of patients. However, the feasibility of such an approach should be studied. Nonetheless, the presence of a $\geq 50 \%$ stenosis remains a simple and powerful distinguisher between high and low risk patients. Moreover, the presence of high risk plaque characteristics can successfully risk stratify individuals in more detail. It is important to realize that the absolute number of events in patients without CAD is very low. Probably, a pragmatic approach towards symptom management seems appropriate in these patients.

\section{CCTA in the clinical work-up}

With every new diagnostic technique being introduced, the method finds itself competing with the conventional work-up of patients. Clinicians will need time to oversee the overall advantages and pitfalls, which will have to be studied. Because of the high negative predictive power of CCTA, it has traditionally been regarded appropriate for low- to intermediate-risk patients. One of the major concerns accompanying CCTA has been radiation dose and the subsequent exposure of cancer risk to the population. However, since the average dose is declining significantly with modern scanners, it can be expected that CCTA will be considered appropriate in lower-risk patients as well. Off course, these times of exponential health-care costs, make cost-effectiveness especially important. Although a single CCTA examination is more expensive than exercise ECG or echocardiography, it is cheaper than conventional angiography or nuclear imaging techniques. CCTA as a gatekeeper before conventional angiography has shown to be cost-effective in low- to intermediate-risk patients. ${ }^{83,84}$ In comparison to cardiovascular magnetic resonance imaging, CCTA has been 
reported to be more cost-effective for all pre-test probabilities. ${ }^{85}$ Min et al. performed a prospective multi-center trial and concluded that a strategy of CCTA alone was more cost-effective than a strategy also incorporating myocardial perfusion imaging in patients with an intermediate pre-test probability. ${ }^{86} \mathrm{Com}-$ parable findings were done by several observational studies. ${ }^{87,88}$

Also, the overall costs were not higher when applying CCTA as the first diagnostic modality in acutely presenting low-risk chest pain patients. ${ }^{89} \mathrm{Im}$ portantly, time spent on the emergency unit for patients undergoing CCTA was lower than for patients subject to standard care. ${ }^{90,}{ }^{91}$ Nevertheless, an accompanying increase in follow-up diagnostics and radiation exposure is still a concern. ${ }^{92}$ However, it is conceivable that by means of directly imaging the coronary arteries, patients without $\mathrm{CAD}$ can be reassured faster and the total amount of clinical contacts and diagnostics will decrease. Also, CCTA seems effective in selecting patients for conventional angiography, subsequently reducing the number of unnecessary invasive catheterizations. ${ }^{91}$ However, for patients with a high pre-test probability, direct conventional angiography has been reported to be the most cost-effective approach. ${ }^{84-86}$

Clinical risk factors as well as chest pain typicality remain poor predictors of significant CAD. We among others have shown that even among high-risk patients, there is a substantial proportion without any CAD. For instance, in patients with a high FRS $(>20 \%), 17 \%$ had no CAD (chapter 2$)$. We have seen during recent years that the diagnostic ability of CCTA increases. Even severe CAD can now be assessed with higher accuracy. These developments will probably pave the way for CCTA to serve as a gatekeeper in higher-risk patients as well, and will help to reclassify and risk stratify an increasing number of chest pain patients. Applying CT perfusion imaging could further maximize risk stratification in this patient group. On the other end of the spectrum, care has to be taken to prevent overuse of diagnostic imaging. Technical advances in medicine often drive costs, this has been especially evident in the United States of America. ${ }^{93}$ One of the biggest issues is that patients as well as doctors pursue a high amount of diagnostic certainty. We will have to bear in mind that the absolute event rates we see in low-risk populations are very low. Although for instance CCTA may have important prognostic implications, its costeffectiveness is far from established in lower-risk patients. This will be an important field of study during the following years. 


\section{Future directions for research}

\section{Assessment of plaque risk}

The ability of CCTA to non-invasively visualize plaque characteristics is a unique feature. These plaque characteristics provide a promising approach to improve risk stratification of patients. We have demonstrated the incremental prognostic value of applying a semi-automated software algorithm to predict for acute coronary syndrome. In contrast to the detection of a single vulnerable plaque, we tried to acknowledge the diffuse character of atherosclerotic disease. The results implicate that such an approach has potential to benefit the identification of the high-risk patient. The plaque cutoff values need to be established in prospective studies in different populations. Moreover, further technical developments in CT might enable the identification of additional plaque characteristics harboring prognostic information. For instance, especially the identification of non-calcified plaque content is problematic to date. The differentiation between fibrous tissue, lipid-rich core, intra-plaque hemorrhage and fibrous cap is still not possible using CCTA, although pathology studies have shown their association with cardiac events. Advances in spatial resolution might improve detection of different components. Also, the application of dual-energy CT, a technique using two X-ray tubes with different tube voltage, might improve tissue characterization. ${ }^{94}$ In addition, functional plaque inflammation can be evaluated using fludeoxyglucose positron emission tomography (FDG-PET). ${ }^{95}$ However, currently PET imaging of coronary arteries is still problematic.

The development of new software algorithms that can automatically quantify CAD on CCTA also merits attention. The labor intensity of semiautomated plaque quantification limits its clinical usefulness. Although automated plaque contouring already is possible, research is needed to establish its value.

Subsequent to risk stratification, quantification of plaque characteristics may also play an important role in the selection of medical therapy. The higher the risk, the more aggressive therapy will be indicated for that individual patient. With an ever broader pharmacological arsenal available, we will need an accurate assessment of risk. With the development of new, expensive agents, CCTA could play a pivotal role in the selection of patients that apply for aggressive medical treatment. Such an example could be the recently developed PCSK9-inhibitors, which showed effective LDL-cholesterol lowering in pa- 
tients that were already on statin treatment. ${ }^{96}$ Further research in that respect could identify patients that benefit most from a certain pharmacological agent. For instance, statin treatment can be effective in lowering atheromatous plaque volumes, as measured with IVUS. ${ }^{97}$ Also, in statins have shown to lower FDG uptake in the ascending aorta and carotid arteries in a randomized study using PET imaging, suggesting a reduction in plaque inflammation..$^{98} \mathrm{~A}$ recent pilot study showed that lipid content of plaques decreased (as measured with in vivo near infrared spectroscopy), even after short term statin treatment. ${ }^{99}$ Similar results have been observed in small CCTA studies, ${ }^{100}$ whereas in calcium score, no reduction has been observed after statin treatment. ${ }^{101}$ Although it is conceivable that by reducing these high risk plaque features, risk for cardiovascular events will decline as well, the prognostic implications have to be studied. An interesting approach would be to select patients with a high risk plaque burden as determined by CCTA, and randomly assign patients to aggressive therapy in order to investigate the potential reduction in both plaque burden as well as event rate.

\section{Functional assessment with CCTA}

The anatomic information provided by CCTA provides limited information about functional significance of a coronary stenosis. The invasive method of fractional flow reserve (FFR) measurement is regularly used to evaluate pressure difference over a coronary lesion. ${ }^{102,} 103$ This method is proven to effectively identify lesions that benefit from revascularization, ${ }^{104}$ and also correlates well with ischemia as detected by functional testing. ${ }^{103}$

However, the method is invasive and expensive. Using the principles of computational flow dynamics, it has become available to calculate FFR values based on a regular CCTA. Initial research has focused on the incremental diagnostic value that CT-FFR can achieve for the detection of obstructive CAD. ${ }^{105}$ The disadvantage is that imaging data have to be analyses by a parallel supercomputer, and the calculations are time consuming. Moreover, patients with rest angina cannot be assessed, as one of the principles is that coronary supply must meet demand at rest.

A much more simple method is measuring the transluminal attenuation gradient. Here, a decline in luminal attenuation correlated well with invasive FFR, and this method might be a promising tool in identifying significant stenoses. ${ }^{106}$ Transluminal attenuation gradient at this moment is only proven feasible using a 320-slide scanner, which is the only modality to scan the entire 
heart within a single gantry rotation. It has to be tested if other fast CT scanners with very low scan times, such as dual source CT, can also reliably perform transluminal attenuation gradients.

These methods likely aid diagnostic accuracy of CCTA, by improving detection of stenotic coronary lesions. Importantly, their application might decrease follow-up diagnostics. However, it remains to be seen whether CTFFR values and/or transluminal attenuation gradients will provide incremental prognostic value in patients presenting with stable chest pain. According to most observations, coronary events occur often in previous non-obstructive CAD. ${ }^{107}$ Our own observations as described in chapter 7 underline this. Therefore, in my opinion it may not be that likely that functional information in nonobstructive CAD will provide additional prognostic information. Moreover, we provide new evidence that quantification of plaque characteristics can improve the prognostic value of CCTA. In the end, this comes back to the issue of anatomical versus functional information. Albeit the functional significance of a coronary lesion beholds beyond doubt important implications in advanced $\mathrm{CAD}$, however, in earlier states of atherosclerosis it is probably anatomic information that is most relevant. In my opinion, it is probable that plaque characteristics can predict plaque rupture and subsequent ACS, while functional information can conclude which segments can benefit from revascularization, in patients with advanced CAD.

\section{Promoting cardiovascular health}

Traditionally, clinical focus has been on the presence of stenotic CAD. However, as this thesis demonstrates, we are able to image important features of atherosclerosis before coronary artery obstruction is present. This issue reflects on the role that cardiology has in society. Are we there to treat advanced CAD, relieving symptoms but never curing patients, all at a high economic burden? Or should we attempt to combat CAD in an earlier stage? In order to reduce the atherosclerotic burden on a population scale we should focus on promoting healthy lifestyle. Such an approach might be most effective in children. ${ }^{108}$ Legislation such as banning smoking from public places have had a tremendous effect on reducing cardiovascular disease. ${ }^{109}$ In our studies, smoking has been a consistently important risk factor for the occurrence of CAD. Importantly, smokers not only put themselves at risk, as also second hand smoke has been shown to increase coronary calcification. ${ }^{110}$ For the average chest pain patient, risk reduction seems particularly important. In my opinion, cardiac imaging 
could have an important role in this process. As current clinical risk scores are inaccurate, imaging can improve risk stratification. This would facilitate a more accurate selection of patients who will benefit from aggressive medical therapy. But it is also a way to inform patients about their own cardiovascular health. Being able to literally see cardiovascular risk might help to motivate improving individual cardiovascular health.

\section{References}

1. Achenbach S, Ulzheimer S, Baum U, et al. Noninvasive coronary angiography by retrospectively ECG-gated multislice spiral CT. Circulation 2000;102:2823-8.

2. Nieman K, Oudkerk M, Rensing BJ, et al. Coronary angiography with multi-slice computed tomography. Lancet 2001;357:599-603.

3. Hoffmann $\mathrm{MH}$, Shi H, Schmitz BL, et al. Noninvasive coronary angiography with multislice computed tomography. JAMA 2005;293:2471-8.

4. Schroeder S, Achenbach S, Bengel F, et al. Cardiac computed tomography: indications, applications, limitations, and training requirements: report of a Writing Group deployed by the Working Group Nuclear Cardiology and Cardiac CT of the European Society of Cardiology and the European Council of Nuclear Cardiology. Eur Heart J 2008;29:531-56.

5. Fazel R, Krumholz HM, Wang Y, et al. Exposure to low-dose ionizing radiation from medical imaging procedures. N Engl J Med 2009;361:849-57.

6. Carpeggiani C, Landi P, Michelassi C, Marraccini P, Picano E. Trends of increasing medical radiation exposure in a population hospitalized for cardiovascular disease (1970-2009). PLoS One 2012;7:e50168.

7. Einstein AJ. Effects of radiation exposure from cardiac imaging: how good are the data? J Am Coll Cardiol 2012;59:553-65.

8. Preston DL, Shimizu Y, Pierce DA, Suyama A, Mabuchi K. Studies of mortality of atomic bomb survivors. Report 13: Solid cancer and noncancer disease mortality: 1950-1997. Radiat Res 2003;160:381-407.

9. Doll R, Wakeford R. Risk of childhood cancer from fetal irradiation. Br J Radiol 1997;70:130-9.

10. Richardson DB, Wing S, Hoffmann W. Cancer risk from low-level ionizing radiation: the role of age at exposure. Occup Med 2001;16:191-218.

11. Cousins C, Miller DL, Bernardi G, et al. ICRP PUBLICATION 120: Radiological protection in cardiology. Ann ICRP 2013;42:1-125. 
12. Sun Z, Ng KH. Prospective versus retrospective ECG-gated multislice CT coronary angiography: a systematic review of radiation dose and diagnostic accuracy. Eur J Radiol 2012;81:e94-100.

13. Raff GL, Chinnaiyan KM, Share DA, et al. Radiation dose from cardiac computed tomography before and after implementation of radiation dose-reduction techniques. JAMA 2009;301:2340-8.

14. Lell M, Marwan M, Schepis T, et al. Prospectively ECG-triggered high-pitch spiral acquisition for coronary CT angiography using dual source CT: technique and initial experience. Eur Radiol 2009;19:2576-83.

15. Achenbach S, Marwan M, Ropers D, et al. Coronary computed tomography angiography with a consistent dose below $1 \mathrm{mSv}$ using prospectively electrocardiogram-triggered highpitch spiral acquisition. Eur Heart J 2010;31:340-6.

16. Winklehner A, Karlo C, Puippe G, et al. Raw data-based iterative reconstruction in body CTA: evaluation of radiation dose saving potential. Eur Radiol 2011;21:2521-6.

17. May MS, Wust W, Brand M, et al. Dose reduction in abdominal computed tomography: intraindividual comparison of image quality of full-dose standard and half-dose iterative reconstructions with dual-source computed tomography. Invest Radiol 2011;46:465-70.

18. Gosling O, Loader R, Venables P, et al. A comparison of radiation doses between state-ofthe-art multislice CT coronary angiography with iterative reconstruction, multislice CT coronary angiography with standard filtered back-projection and invasive diagnostic coronary angiography. Heart 2010;96:922-6.

19. Coles DR, Smail MA, Negus IS, et al. Comparison of radiation doses from multislice computed tomography coronary angiography and conventional diagnostic angiography. J Am Coll Cardiol 2006;47:1840-5.

20. Hausleiter J, Meyer T, Hermann F, et al. Estimated radiation dose associated with cardiac CT angiography. JAMA 2009;301:500-7.

21. Sandborg M, Fransson SG, Pettersson H. Evaluation of patient-absorbed doses during coronary angiography and intervention by femoral and radial artery access. Eur Radiol 2004;14:653-8.

22. Einstein AJ, Moser KW, Thompson RC, Cerqueira MD, Henzlova MJ. Radiation dose to patients from cardiac diagnostic imaging. Circulation 2007;116:1290-305.

23. Slomka PJ, Dey D, Duvall WL, Henzlova MJ, Berman DS, Germano G. Advances in nuclear cardiac instrumentation with a view towards reduced radiation exposure. Curr Cardiol Rep 2012;14:208-16.

24. Goodman SN. Toward evidence-based medical statistics. 2: The Bayes factor. Ann Intern Med 1999;130:1005-13.

25. Garcia MJ, Lessick J, Hoffmann MH. Accuracy of 16-row multidetector computed tomography for the assessment of coronary artery stenosis. JAMA 2006;296:403-11. 
26. Budoff MJ, Dowe D, Jollis JG, et al. Diagnostic performance of 64-multidetector row coronary computed tomographic angiography for evaluation of coronary artery stenosis in individuals without known coronary artery disease: results from the prospective multicenter ACCURACY (Assessment by Coronary Computed Tomographic Angiography of Individuals Undergoing Invasive Coronary Angiography) trial. J Am Coll Cardiol 2008;52:1724-32.

27. Taylor AJ, Cerqueira M, Hodgson JM, et al. ACCF/SCCT/ACR/AHA/ASE/ASNC/NASCI/SCAI/SCMR 2010 appropriate use criteria for cardiac computed tomography: a report of the American College of Cardiology Foundation Appropriate Use Criteria Task Force, the Society of Cardiovascular Computed Tomography, the American College of Radiology, the American Heart Association, the American Society of Echocardiography, the American Society of Nuclear Cardiology, the North American Society for Cardiovascular Imaging, the Society for Cardiovascular Angiography and Interventions, and the Society for Cardiovascular Magnetic Resonance. J Am Coll Cardiol 2010;56:1864-94.

28. Vanhecke TE, Madder RD, Weber JE, Bielak LF, Peyser PA, Chinnaiyan KM. Development and validation of a predictive screening tool for uninterpretable coronary CT angiography results. Circ Cardiovasc Imaging 2011;4:490-7.

29. Miller JM, Rochitte CE, Dewey M, et al. Diagnostic performance of coronary angiography by 64-row CT. N Engl J Med 2008;359:2324-36.

30. Meijboom WB, Meijs MF, Schuijf JD, et al. Diagnostic accuracy of 64-slice computed tomography coronary angiography: a prospective, multicenter, multivendor study. J Am Coll Cardiol 2008;52:2135-44.

31. Zhang S, Levin DC, Halpern EJ, Fischman D, Savage M, Walinsky P. Accuracy of MDCT in assessing the degree of stenosis caused by calcified coronary artery plaques. AJR Am J Roentgenol 2008;191:1676-83.

32. Alkadhi H, Stolzmann P, Desbiolles L, et al. Low-dose, 128-slice, dual-source CT coronary angiography: accuracy and radiation dose of the high-pitch and the step-and-shoot mode. Heart 2010;96:933-8.

33. Achenbach S, Goroll T, Seltmann M, et al. Detection of coronary artery stenoses by lowdose, prospectively ECG-triggered, high-pitch spiral coronary CT angiography. JACC Cardiovasc Imaging 2011;4:328-37.

34. Min JK, Shaw LJ, Devereux RB, et al. Prognostic value of multidetector coronary computed tomographic angiography for prediction of all-cause mortality. J Am Coll Cardiol 2007;50:1161-70.

35. Hadamitzky M, Freissmuth B, Meyer T, et al. Prognostic value of coronary computed tomographic angiography for prediction of cardiac events in patients with suspected coronary artery disease. JACC Cardiovasc Imaging 2009;2:404-11.

36. Min JK, Dunning A, Lin FY, et al. Age- and sex-related differences in all-cause mortality risk based on coronary computed tomography angiography findings results from the International Multicenter CONFIRM (Coronary CT Angiography Evaluation for Clinical Outcomes: An International Multicenter Registry) of 23,854 patients without known coronary artery disease. J Am Coll Cardiol 2011;58:849-60. 
37. Hadamitzky M, Distler R, Meyer T, et al. Prognostic value of coronary computed tomographic angiography in comparison with calcium scoring and clinical risk scores. Circ Cardiovasc Imaging 2011;4:16-23.

38. Santoro GM, Sciagra R, Buonamici P, et al. Head-to-head comparison of exercise stress testing, pharmacologic stress echocardiography, and perfusion tomography as first-line examination for chest pain in patients without history of coronary artery disease. J Nucl Cardiol 1998;5:19-27.

39. Schaap J, Kauling RM, Boekholdt SM, et al. Incremental diagnostic accuracy of hybrid SPECT/CT coronary angiography in a population with an intermediate to high pre-test likelihood of coronary artery disease. Eur Heart J Cardiovasc Imaging 2013;14:642-9.

40. Hulten EA, Carbonaro S, Petrillo SP, Mitchell JD, Villines TC. Prognostic Value of Cardiac Computed Tomography Angiography A Systematic Review and Meta-Analysis. J Am Coll Cardiol 2011;57:1237-47.

41. Hur J, Kim YJ, Lee HJ, et al. Quantification and characterization of obstructive coronary plaques using 64-slice computed tomography: a comparison with intravascular ultrasound. J Comput Assist Tomogr 2009;33:186-92.

42. Pundziute G, Schuijf JD, Jukema JW, et al. Head-to-head comparison of coronary plaque evaluation between multislice computed tomography and intravascular ultrasound radiofrequency data analysis. JACC Cardiovasc Interv 2008;1:176-82.

43. Sun J, Zhang Z, Lu B, et al. Identification and quantification of coronary atherosclerotic plaques: a comparison of 64-MDCT and intravascular ultrasound. AJR Am J Roentgenol 2008;190:748-54.

44. Voros S, Rinehart S, Qian Z, et al. Prospective validation of standardized, 3-dimensional, quantitative coronary computed tomographic plaque measurements using radiofrequency backscatter intravascular ultrasound as reference standard in intermediate coronary arterial lesions: results from the ATLANTA (assessment of tissue characteristics, lesion morphology, and hemodynamics by angiography with fractional flow reserve, intravascular ultrasound and virtual histology, and noninvasive computed tomography in atherosclerotic plaques) I study. JACC Cardiovasc Interv 2011;4:198-208.

45. Leber AW, Becker A, Knez A, et al. Accuracy of 64-slice computed tomography to classify and quantify plaque volumes in the proximal coronary system: a comparative study using intravascular ultrasound. J Am Coll Cardiol 2006;47:672-7.

46. Hoffmann U, Moselewski F, Nieman K, et al. Noninvasive assessment of plaque morphology and composition in culprit and stable lesions in acute coronary syndrome and stable lesions in stable angina by multidetector computed tomography. J Am Coll Cardiol 2006;47:1655-62.

47. Kitagawa T, Yamamoto H, Horiguchi J, et al. Characterization of noncalcified coronary plaques and identification of culprit lesions in patients with acute coronary syndrome by 64-slice computed tomography. JACC Cardiovasc Imaging 2009;2:153-60. 
48. Pundziute G, Schuijf JD, Jukema JW, et al. Evaluation of plaque characteristics in acute coronary syndromes: non-invasive assessment with multi-slice computed tomography and invasive evaluation with intravascular ultrasound radiofrequency data analysis. Eur Heart J 2008;29:2373-81.

49. Sato A, Ohigashi H, Nozato T, et al. Coronary artery spatial distribution, morphology, and composition of nonculprit coronary plaques by 64-slice computed tomographic angiography in patients with acute myocardial infarction. Am J Cardiol 2010;105:930-5.

50. Hammer-Hansen S, Kofoed KF, Kelbaek H, et al. Volumetric evaluation of coronary plaque in patients presenting with acute myocardial infarction or stable angina pectoris-a multislice computerized tomography study. Am Heart J 2009;157:481-7.

51. Schuijf JD, Beck T, Burgstahler C, et al. Differences in plaque composition and distribution in stable coronary artery disease versus acute coronary syndromes; non-invasive evaluation with multi-slice computed tomography. Acute Card Care 2007;9:48-53.

52. Motoyama S, Kondo T, Sarai M, et al. Multislice computed tomographic characteristics of coronary lesions in acute coronary syndromes. J Am Coll Cardiol 2007;50:319-26.

53. Pflederer T, Marwan M, Schepis T, et al. Characterization of culprit lesions in acute coronary syndromes using coronary dual-source CT angiography. Atherosclerosis 2011;211:43744.

54. Motoyama S, Sarai M, Harigaya H, et al. Computed tomographic angiography characteristics of atherosclerotic plaques subsequently resulting in acute coronary syndrome. J Am Coll Cardiol 2009;54:49-57.

55. Matsumoto N, Sato Y, Yoda S, et al. Prognostic value of non-obstructive CT low-dense coronary artery plaques detected by multislice computed tomography. Circ J 2007;71:1898903.

56. Kristensen TS, Kofoed KF, Kuhl JT, Nielsen WB, Nielsen MB, Kelbaek H. Prognostic implications of nonobstructive coronary plaques in patients with non-ST-segment elevation myocardial infarction: a multidetector computed tomography study. J Am Coll Cardiol 2011;58:502-9.

57. Stone GW, Maehara A, Lansky AJ, et al. A prospective natural-history study of coronary atherosclerosis. N Engl J Med 2011;364:226-35.

58. Schaar JA, Muller JE, Falk E, et al. Terminology for high-risk and vulnerable coronary artery plaques. Report of a meeting on the vulnerable plaque, June 17 and 18, 2003, Santorini, Greece. Eur Heart J 2004;25:1077-82.

59. Virmani R, Burke AP, Farb A, Kolodgie FD. Pathology of the vulnerable plaque. J Am Coll Cardiol 2006;47:C13-8.

60. Mann J, Davies MJ. Mechanisms of progression in native coronary artery disease: role of healed plaque disruption. Heart 1999;82:265-8. 
61. Leber AW, Knez A, von Ziegler F, et al. Quantification of obstructive and nonobstructive coronary lesions by 64-slice computed tomography: a comparative study with quantitative coronary angiography and intravascular ultrasound. J Am Coll Cardiol 2005;46:147-54.

62. Cademartiri F, Mollet NR, Runza G, et al. Influence of intracoronary attenuation on coronary plaque measurements using multislice computed tomography: observations in an ex vivo model of coronary computed tomography angiography. Eur Radiol 2005;15:1426-31.

63. Achenbach S, Boehmer K, Pflederer T, et al. Influence of slice thickness and reconstruction kernel on the computed tomographic attenuation of coronary atherosclerotic plaque. J Cardiovasc Comput Tomogr 2010;4:110-5.

64. Cademartiri F, Runza G, Palumbo A, et al. Lumen enhancement influences absolute noncalcific plaque density on multislice computed tomography coronary angiography: ex-vivo validation and in-vivo demonstration. J Cardiovasc Med (Hagerstown) 2010;11:337-44.

65. Maurovich-Horvat $\mathrm{P}$, Schlett CL, Alkadhi H, et al. The napkin-ring sign indicates advanced atherosclerotic lesions in coronary CT angiography. JACC Cardiovasc Imaging 2012;5:1243-52.

66. Arbustini E, Dal Bello B, Morbini P, et al. Plaque erosion is a major substrate for coronary thrombosis in acute myocardial infarction. Heart 1999;82:269-72.

67. Farb A, Burke AP, Tang AL, et al. Coronary plaque erosion without rupture into a lipid core. A frequent cause of coronary thrombosis in sudden coronary death. Circulation 1996;93:1354-63.

68. Burke AP, Farb A, Malcom GT, Liang Y, Smialek J, Virmani R. Effect of risk factors on the mechanism of acute thrombosis and sudden coronary death in women. Circulation 1998;97:2110-6.

69. Virmani R, Kolodgie FD, Burke AP, Farb A, Schwartz SM. Lessons from sudden coronary death: a comprehensive morphological classification scheme for atherosclerotic lesions. Arterioscler Thromb Vasc Biol 2000;20:1262-75.

70. Versteylen MO, Kietselaer BL, Dagnelie PC, et al. Additive value of semi-automated quantification of coronary artery disease using cardiac CT-angiography to predict for future acute coronary syndrome. J Am Coll Cardiol 2013;61:2296-305.

71. Finn AV, Nakano M, Narula J, Kolodgie FD, Virmani R. Concept of vulnerable/unstable plaque. Arterioscler Thromb Vasc Biol 2010;30:1282-92.

72. Borissoff JI, Joosen IA, Versteylen MO, Spronk HM, ten Cate H, Hofstra L. Accelerated in vivo thrombin formation independently predicts the presence and severity of CT angiographic coronary atherosclerosis. JACC Cardiovasc Imaging 2012;5:1201-10.

73. Laufer EM, Mingels AM, Winkens MH, et al. The extent of coronary atherosclerosis is associated with increasing circulating levels of high sensitive cardiac troponin T. Arterioscler Thromb Vasc Biol 2010;30:1269-75. 
74. Mingels AM, Joosen IA, Versteylen MO, et al. High-sensitivity cardiac troponin T: risk stratification tool in patients with symptoms of chest discomfort. PLoS One 2012; 7:e35059.

75. Ostrom MP, Gopal A, Ahmadi N, et al. Mortality incidence and the severity of coronary atherosclerosis assessed by computed tomography angiography. J Am Coll Cardiol 2008;52:1335-43.

76. Proudfit WL, Shirey EK, Sones FM, Jr. Selective cine coronary arteriography. Correlation with clinical findings in 1,000 patients. Circulation 1966;33:901-10.

77. CASS Principal lnvestigators. Coronary artery surgery study (CASS): a randomized trial of coronary artery bypass surgery. Survival data. Circulation 1983;68:939-50.

78. Mintz GS, Painter JA, Pichard AD, et al. Atherosclerosis in angiographically "normal" coronary artery reference segments: an intravascular ultrasound study with clinical correlations. J Am Coll Cardiol 1995;25:1479-85.

79. Erbel R, Ge J, Bockisch A, et al. Value of intracoronary ultrasound and Doppler in the differentiation of angiographically normal coronary arteries: a prospective study in patients with angina pectoris. Eur Heart J 1996;17:880-9.

80. Bugiardini R, Bairey Merz CN. Angina with "normal" coronary arteries: a changing philosophy. JAMA 2005;293:477-84.

81. Bugiardini R, Manfrini O, Pizzi C, Fontana F, Morgagni G. Endothelial function predicts future development of coronary artery disease: a study of women with chest pain and normal coronary angiograms. Circulation 2004;109:2518-23.

82. Vane JR, Anggard EE, Botting RM. Regulatory functions of the vascular endothelium. N Engl J Med 1990;323:27-36.

83. Genders TS, Meijboom WB, Meijs MF, et al. CT coronary angiography in patients suspected of having coronary artery disease: decision making from various perspectives in the face of uncertainty. Radiology 2009;253:734-44.

84. Dorenkamp M, Bonaventura K, Sohns C, Becker CR, Leber AW. Direct costs and costeffectiveness of dual-source computed tomography and invasive coronary angiography in patients with an intermediate pretest likelihood for coronary artery disease. Heart 2012;98:460-7.

85. Dewey M, Hamm B. Cost effectiveness of coronary angiography and calcium scoring using CT and stress MRI for diagnosis of coronary artery disease. Eur Radiol 2007;17:1301-9.

86. Min JK, Gilmore A, Budoff MJ, Berman DS, O'Day K. Cost-effectiveness of coronary CT angiography versus myocardial perfusion SPECT for evaluation of patients with chest pain and no known coronary artery disease. Radiology 2010;254:801-8.

87. Min JK, Kang N, Shaw LJ, et al. Costs and clinical outcomes after coronary multidetector CT angiography in patients without known coronary artery disease: comparison to myocardial perfusion SPECT. Radiology 2008;249:62-70. 
88. Min JK, Shaw LJ, Berman DS, Gilmore A, Kang N. Costs and clinical outcomes in individuals without known coronary artery disease undergoing coronary computed tomographic angiography from an analysis of Medicare category III transaction codes. Am J Cardiol 2008;102:672-8.

89. Goldstein JA, Chinnaiyan KM, Abidov A, et al. The CT-STAT (Coronary Computed Tomographic Angiography for Systematic Triage of Acute Chest Pain Patients to Treatment) trial. J Am Coll Cardiol 2011;58:1414-22.

90. Goldstein JA, Gallagher MJ, O'Neill WW, Ross MA, O'Neil BJ, Raff GL. A randomized controlled trial of multi-slice coronary computed tomography for evaluation of acute chest pain. J Am Coll Cardiol 2007;49:863-71.

91. Litt HI, Gatsonis C, Snyder B, et al. CT angiography for safe discharge of patients with possible acute coronary syndromes. N Engl J Med 2012;366:1393-403.

92. Hoffmann U, Truong QA, Schoenfeld DA, et al. Coronary CT angiography versus standard evaluation in acute chest pain. N Engl J Med 2012;367:299-308.

93. Smith-Bindman R, Miglioretti DL, Larson EB. Rising use of diagnostic medical imaging in a large integrated health system. Health Aff (Millwood) 2008;27:1491-502.

94. Johnson TR, Krauss B, Sedlmair M, et al. Material differentiation by dual energy CT: initial experience. Eur Radiol 2007;17:1510-7.

95. Abdelbaky A, Corsini E, Figueroa AL, et al. Focal Arterial Inflammation Precedes Subsequent Calcification in the Same Location: A Longitudinal FDG-PET/CT Study. Circ Cardiovasc Imaging 2013;6:747-54.

96. Roth EM, McKenney JM, Hanotin C, Asset G, Stein EA. Atorvastatin with or without an antibody to PCSK9 in primary hypercholesterolemia. N Engl J Med 2012;367:1891-900.

97. Nissen SE, Nicholls SJ, Sipahi I, et al. Effect of very high-intensity statin therapy on regression of coronary atherosclerosis: the ASTEROID trial. JAMA 2006;295:1556-65.

98. Tawakol A, Fayad ZA, Mogg R, et al. Intensification of Statin Therapy Results in a Rapid Reduction in Atherosclerotic Inflammation: Results of A Multi-Center FDG-PET/CT Feasibility Study. J Am Coll Cardiol 2013;62:909-17.

99. Kini AS, Baber U, Kovacic JC, et al. Changes in Plaque Lipid Content after Short-Term, Intensive versus Standard Statin Therapy: The YELLOW Trial. J Am Coll Cardiol 2013;62:21-9.

100. Inoue K, Motoyama S, Sarai M, et al. Serial coronary CT angiography-verified changes in plaque characteristics as an end point: evaluation of effect of statin intervention. JACC Cardiovasc Imaging 2010;3:691-8.

101. Schmermund A, Achenbach S, Budde T, et al. Effect of intensive versus standard lipidlowering treatment with atorvastatin on the progression of calcified coronary atherosclerosis over 12 months: a multicenter, randomized, double-blind trial. Circulation 2006;113:427-37. 
102. Pijls NH, van Son JA, Kirkeeide RL, De Bruyne B, Gould KL. Experimental basis of determining maximum coronary, myocardial, and collateral blood flow by pressure measurements for assessing functional stenosis severity before and after percutaneous transluminal coronary angioplasty. Circulation 1993;87:1354-67.

103. Pijls NH, De Bruyne B, Peels K, et al. Measurement of fractional flow reserve to assess the functional severity of coronary-artery stenoses. N Engl J Med 1996;334:1703-8.

104. De Bruyne B, Pijls NH, Kalesan B, et al. Fractional flow reserve-guided PCI versus medical therapy in stable coronary disease. N Engl J Med 2012;367:991-1001.

105. Min JK, Leipsic J, Pencina MJ, et al. Diagnostic accuracy of fractional flow reserve from anatomic CT angiography. JAMA 2012;308:1237-45.

106. Wong DT, Ko BS, Cameron JD, et al. Transluminal attenuation gradient in coronary computed tomography angiography is a novel noninvasive approach to the identification of functionally significant coronary artery stenosis: a comparison with fractional flow reserve. J Am Coll Cardiol 2013;61:1271-9.

107. Ambrose JA, Tannenbaum MA, Alexopoulos D, et al. Angiographic progression of coronary artery disease and the development of myocardial infarction. J Am Coll Cardiol 1988;12:56-62.

108. Farkouh ME, Boden WE, Bittner V, et al. Risk Factor Control for CAD Secondary Prevention in Large Randomized Trials. J Am Coll Cardiol 2013;61:1607-15.

109. Meyers DG, Neuberger JS, He J. Cardiovascular effect of bans on smoking in public places: a systematic review and meta-analysis. J Am Coll Cardiol 2009;54:1249-55.

110. Yankelevitz DF, Henschke CI, Yip R, et al. Second-Hand Tobacco Smoke in Never Smokers Is a Significant Risk Factor for Coronary Artery Calcification. JACC Cardiovasc Imaging 2013;6:651-7. 


\section{Summary}

This thesis provides new insights in the clinical application of cardiac computed tomographic angiography (CCTA), and its implications for cardiovascular risk stratification. Initial risk assessment in stable chest pain patients is often based on traditional risk factors and type of chest pain. However, we demonstrate in chapter 2 that the accuracy for clinical risk scores to predict for coronary artery disease (CAD) is rather moderate. Methods to improve risk stratification are therefore desirable. Such an approach could be the application of imaging techniques. One well described marker of CAD is calcium score. Calcium score can be calculated using a very low dose, non-contrast enhanced scan. However, also the amount of epicardial adipose tissue is visible on this scan, and has been associated with presence of CAD. We investigate this association in chapter 3, however, conclude that it could not provide incremental predictive value on top of traditional risk factors.

In addition, patients with coronary anomalies have been described to be at risk of sudden death, especially in the presence of an inter-arterial course. In chapter 4, we describe different types of coronary anomalies that were detected with CCTA. The short-term follow-up is good in this adult population, both in anomalies with and without a inter-arterial course.

The direct imaging of CAD using CCTA is further investigated in a series of prognostic studies. Importantly, we show an increment in prognostic value when adding CCTA to the clinical work-up of Framingham risk score, exercise ECG and calcium score. In chapter 5, we demonstrate that in an intermediate risk population, CCTA is a suitable follow-up diagnostic and can aid prediction of cardiac events. In chapter 6 we investigate the difference in prognostic value of CCTA between men and women. Although overall prognostic value of CCTA is robust in both men and women, we demonstrate that women with a normal CCTA develop events more often as compared with men. We hypothesize that this could be based on different mechanisms resulting in cardiac events in women. Although the event rates are low, these findings are hypothesis-generating and might implicate a limitation for the prognostic value of CCTA in women.

Currently, the assessment of CCTA is often limited to description of stenosis severity. However, CCTA is able to visualize a broader spectrum of the vessel wall, potentially including coronary plaque characteristics. Since cardiac 
events are often not based on stenotic lesions, but on plaques that are prone to provoke intracoronary thrombosis (e.g. via plaque rupture), we hypothesized that the prognostic value of CCTA could be maximized. Indeed, in chapter 7 several plaque characteristics that were associated with the occurrence of acute coronary syndrome are identified. Moreover, we demonstrate that semiautomated quantification of the coronary artery tree provides incremental prognostic value over the conventional assessment of CCTA. Hence, incremental prognostic value is present in CCTA datasets, but not used in daily practice. In other words, the prognostic value of CCTA in patients with CAD can be maximized by using plaque quantification software.

Finally, we investigate serum chemokines to predict for CAD as well as cardiac events in chapter 8 . Although we have demonstrated that CCTA is able to image coronary risk to a certain extent, risk cannot exclusively be defined by anatomic substrate. For instance, it is conceivable that also factors as thrombogenicity and plaque inflammation will contribute to the risk for an atherosclerotic event. We conclude that serum marker CC chemokine ligand 5 has independent value to predict for $\mathrm{CAD}$ as well as for cardiac events. Identification of such biomarkers could provide a cheap and simple contribution to risk stratification of patients suspected for CAD. 


\section{Nederlandse samenvatting}

Dit proefschrift onderzoekt de klinische toepassing van cardiale computer tomografische angiografie (CCTA), en zijn invloed op cardiovasculaire risicostratificatie. Het inschatten van het risico op coronairlijden bij patiënten met stabiele pijn op de borst gebeurt initieel door middel van traditionele risicofactoren en type pijn. In hoofdstuk 2 laten we zien dat de nauwkeurigheid waarin klinisch risico scores coronairlijden kunnen voorspellen, matig is. Betere risicostratificatie lijkt daarom gewenst. Dat zou onder andere kunnen met beeldvormende technieken. Een gevestigde indicator van coronairlijden is calcium score. Calcium score is te verkrijgen door middel van een eenvoudige scan zonder contrast. Maar ook de hoeveelheid epicardiaal vetweefsel is zichtbaar op een dergelijke scan, en lijkt geassocieerd te zijn met coronairlijden. We onderzoeken dit in hoofdstuk 3 , maar concluderen dat deze meting geen toegevoegde waarde kan bieden bovenop traditionele risicofactoren.

Aangeboren afwijkingen aan het beloop van coronairarteriën, oftewel coronaire anomalieën, kunnen door CCTA goed worden afgebeeld. Ze worden echter ook geassocieerd met plotse hartdood, vooral indien er sprake is van een inter-arterieel verloop. Het belang van deze 'toevalsbevinding' op CCTA wordt onderzocht in hoofdstuk 4. De kortetermijn follow-up in deze volwassen populatie is echter goed, zowel in patiënten met inter-arteriële als niet-inter-arteriële anomalieën.

Daarnaast onderzochten we het vermogen van CCTA om te voorspellen voor cardiale events (o.a. hartinfarct). In hoofdstuk 5 tonen we de toegevoegde prognostische waarde aan van CCTA bovenop Framingham risico score, inspannings-ECG en calcium score. CCTA kan het voorspellen van cardiale events in mensen met pijn op de borst dus verbeteren.

In hoofdstuk 6 wordt onderzocht of er verschil is in prognostische waarde van CCTA tussen mannen en vrouwen. Het is bekend dat vrouwen met een cardiaal event vaak minder uitgebreid coronairlijden hebben in vergelijking met mannen met events. Waar het merendeel van de events ontstaat door ruptureren van een plaque, komt het mechanisme van plaque erosie relatief vaker voor bij vrouwen. In het algemeen gaat het hier om kleinere plaques. Wat we demonstreren is dat vrouwen zonder zichtbaar coronairlijden op CCTA vaker events ontwikkelen in vergelijking met mannen. Van belang is dat het hier gaat 
om een zeer lage kans op events, en de prognostische waarde van CCTA goed is in zowel mannen als vrouwen.

Desondanks is de conventionele methode om CCTA te beoordelen meestal beperkt tot het bepalen van de ernst van de vernauwing van de kransslagader. Dit terwijl CCTA ook de vaatwand, en mogelijk de verschillende componenten van coronaire plaques afbeeldt. Voorgaand onderzoek laat zien dat cardiale events vaak niet gebaseerd zijn op langzaam ontstane vernauwingen, maar op de acute vorming van een bloedprop op een plaque. De kans hierop zou mogelijk worden bepaald door de kenmerken van de plaque. Dientengevolge zou de prognostische waarde van CCTA vergroot kunnen worden, door naast stenosegraad ook plaque karakteristieken te meten. In hoofdstuk 7 tonen we aan dat er verschillende karakteristieken van coronairlijden geassocieerd zijn met het optreden van acuut coronair syndroom (ACS). Bovendien laten we zien dat het kwantificeren van die kenmerken met semiautomatische software, toegevoegde waarde biedt bovenop de conventionele CCTA beoordeling. We demonstreren hiermee dat de prognostische waarde van CCTA gemaximaliseerd kan worden, door simpelweg extra softwarematige metingen uit te voeren.

Tot slot onderzoeken we het vermogen van verschillende inflammatoire serum chemokines om coronairlijden, alsmede cardiale events te voorspellen. We lieten eerder zien dat CCTA het cardiale risico in zekere mate kan voorspellen. Het is echter onwaarschijnlijk dat dit risico alleen door het anatomische substraat bepaald wordt. Factoren als tromboseneiging en plaque inflammatie dragen daar immers ook aan bij. We concluderen dat serum marker CC chemokine ligand 5 onafhankelijke voorspellende waarde heeft om coronairlijden alsmede cardiale events te voorspellen. De identificatie van dergelijke 'biomarkers' zou een goedkope en patiëntvriendelijke bijdrage kunnen leveren aan de risicostratificatie van patiënten, en wellicht kunnen bijdragen aan de selectie van patiënten geschikt voor verdere cardiale beeldvorming. 


\section{Nawoord}

Het voltooien van dit proefschrift betekent automatisch het einde van de tijd als promovendus, en met veel plezier kijk ik terug op deze afgelopen jaren. Het is een periode geworden waar zelfontplooiing en samenwerking centraal stonden. Het is niet zwaar of moeilijk geweest, toch vervult de afronding van dit project me met blijdschap en zelfs trots.

Als de dag van gisteren kan ik me mijn eerste werkdag herinneren. Nadat ik bij Ward en Ivo op de kamer was neergezet, de laptop geïnstalleerd had en de koffie gehaald was, stelde ik de vraag 'wordt er nog wat van mij verwacht?' Nog steeds vangt dit wel de essentie van de start van mijn promotietraject, je begint vol enthousiasme, maar eigenlijk heb je geen idee waaraan.

Professor Hofstra, beste Leonard, laat me beginnen bij jou. Je enthousiasme werkt aanstekelijk, en heeft me in grote maten beïnvloed de keuze te maken te starten met het promotietraject. Met je talent mensen bij elkaar te brengen, originele onderzoeksvragen en ongelimiteerd positivisme heb je veel mogelijkheden gecreëerd waar ik gedurende het onderzoek van heb kunnen profiteren. De samenwerking vroeg wel zelfstandigheid en soms was er gebrek aan strakke structuur, maar achteraf heeft het me goed gepast.

Professor Crijns, beste Harry, u kwam in beeld toen Leo vertrok uit het MUMC. Ik kan me goed herinneren dat Ivo en ik ontboden werden op uw kantoor. Even bekroop me de gedachte dat ik mijn baan misschien wel kwijt was, maar niets bleek minder waar. Gedurende het tweewekelijks overleg was altijd volop ruimte voor discussie. Uw ervaring met klinisch onderzoek heeft mij erg geholpen, tegelijkertijd zag ik u toenemend enthousiast worden over cardiale beeldvorming. Ik heb de samenwerking als zeer prettig ervaren.

Bas, ook jouw rol als copromotor is pas ingegaan ongeveer halverwege het promotietraject. Het is natuurlijk fantastisch geweest dat er snel weer een cardioloog op het cardiale CT programma kwam te staan. Tal van praktische zaken heb je onder je hoede genomen, en bovendien heb je goed kunnen helpen met het schrijven en publiceren van de laatste (maar niet de minste!) manuscripten.

Marco, ook jou wil ik graag bedanken voor de prettige samenwerking. Ik heb het geluk gehad dat toen ik startte, de samenwerking tussen cardiologie en radiologie op CT gebied al in volle bloei was. Jouw inzet en interesse in wetenschappelijk onderzoek heeft er verder aan bijgedragen dat zowel de radiologie 
als cardiologie maximaal van dit samenwerkingsverband hebben kunnen profiteren.

Professor Wildberger, beste Joachim, datzelfde geld natuurlijk in grote mate voor $u$. Mijn proefschrift is het product van samenwerking, en daar wil ik $\mathrm{u}$ graag voor bedanken. Voor $\mathrm{u}$ is er nooit een verschil geweest tussen cardiologie en radiologie, en $u$ hebt mij ook altijd maximaal betrokken bij de afdeling radiologie. De CT kamer is dan ook mijn tweede thuis geweest gedurende het onderzoek. Praktische bijstand kwam er ook zeker van de röntgenlaboranten. Soms verveelden we jullie met bloedafnames, informed consents en veranderingen aan het scanprotocol, maar het heeft het onderzoek enorm geholpen. Ook alle studenten die zich hebben geholpen met het bijhouden van de CT database wil ik graag bedanken voor de vrijwillige inzet.

Ivo, niet voor niets promoveren we op dezelfde dag, we hebben het traject als duo doorlopen. Soms is dat een afgelegen bestaan geweest in onze kamer in UNS 50 (door Elmer zelfs geduid als "the Batcave"), wat ook de nodige rust opleverde en waar daarom vaak productieve uren gedraaid werden. Jij bewaakte als geen ander het structurele werkritme, en ik denk dat we elkaar goed hebben aangevuld. Jouw droge humor, koffie, youtube filmpjes tegen de afterlunch-dip, en soms ook het mateloos hard doorwerken aan het complementeren van datasheets, alles heeft bijgedragen aan deze mooie tijd! Aan Sibel en Casper de taak de research kamer te behouden, en natuurlijk ook het mooie onderzoek voort te zetten!

Ook wil ik het cardiac CT team van het eerste uur bedanken. Ward Laufer en Mark Winkens, jullie hebben mateloos veel energie gestoken in het opstarten van het Maastrichtse CT programma, en dankzij jullie ben ik daar ook bij betrokken geraakt. Ook de mogelijkheden bij te dragen aan de cardiac CT cursus heb ik altijd erg gewaardeerd!

Professor Gorgels en Dr Bekkers, beste Ton en Bas, dankzij jullie is mijn wetenschapsstage een succes geworden. Het onderzoek doen, het presenteren op een internationale vergadering, het smaakte naar meer. Het waren vele uren die we daarna nog besteedden aan het publiceren van het manuscript, maar ik heb er enorm van geleerd en daar tijdens het promoveren weer van geprofiteerd.

Mijn collega's van cardiologie, radiologie, klinische chemie, pathologie, statistiek, epidemiologie, ik wil iedereen bedanken voor de samenwerking. In het bijzonder nog Mat Daemen, Wiro Niessen en iedereen van het PARISk team. Met de nodige discussie en soms ook scepsis, werd ik als coronairen- 
onderzoeker opgenomen in een carotiden-consortium. Ik voelde me initieel als een vreemde eend in een nog vreemdere bijt, maar het resultaat van deze samenwerking (beschreven in hoofdstuk 7) vormt nu wel mooi de parel van dit proefschrift. Ook wil ik graag Koen Nieman en Admir Dedic bedanken voor de open samenwerking en jullie hulp bij de totstandkoming van dit hoofdstuk. Dear professor Narula and professor Hecht, thanks for having me at Mount Sinai Hospital, and letting me participate in the cardiac CT/MRI lab. Although the research performed here could not be included in this dissertation, the experience has been great and very valuable!

Roy, de cover en diverse illustraties waren er nooit gekomen zonder jouw creatieve inzet! Dan rest me nog even stil te staan bij de mensen die niet aan dit proefschrift hebben meegeholpen. Kortom, familie, vrienden en vriendinnen. De feestjes, borrels, weekenden en vakanties, het is altijd een enorm plezier. Dan de paranimfen, ik hoop dat jullie niet echt hebben geloofd dat het gebruikelijk is dat jullie enkele vragen voor jullie rekening nemen. Het heeft jullie waarschijnlijk veel tijd gekost om je helemaal in te lezen in dit proefschrift, maar het wordt gewaardeerd, laat de moeilijke vragen nu maar komen!

Ook wil ik even stilstaan bij mijn lieve familie en in het bijzonder het gezin. Rob, Mariëtte, Casper en Maud. Ik heb het gevoel door jullie altijd erg vrij gelaten te zijn, en heb thuis altijd veel ruimte gekregen. We doen allemaal waar we zelf zin in hebben, toch hebben we een hechte band, en is het samenzijn altijd erg ontspannen.

Anouk, jou wil ik graag bedanken. Jij bent altijd vrolijk en de levenslust spat ervan af bij jou. Thuiskomen is altijd meteen genieten, ontspannen en lachen. Het zijn met jou fantastische jaren geweest op de Wolfstraat, en ik hoop dat er nog veel gelukkige jaren mogen volgen! 



\section{Curriculum vitae}

Mathijs Versteylen was born on April 28 th 1984 in Rotterdam, The Netherlands. In 2002, he completed his pre-university education at the Onze Lieve Vrouwelyceum in Breda. That same year he started medical training at Maastricht University. After several electives in cardiology, he accomplished his research elective under supervision of Prof dr. Gorgels in 2009. After obtaining his medical degree, he started as a $\mathrm{PhD}$ fellow under supervision of Prof. dr. Hofstra and Prof. dr. Crijns. In close collaboration

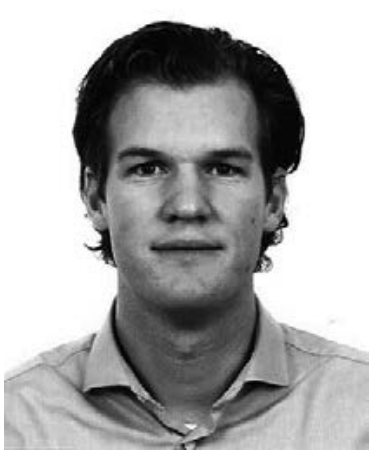
with the radiology department, he specialized in performance and assessment of cardiac CT angiography (over 2000 cases) and assisted in the cardiac CT course (CIRC) in collaboration with Philips Healthcare. Results of studies on cardiac CT were presented on multiple international conferences. In 2013, he spent several months at the cardiac CT/MRI lab of the Mount Sinai Hospital in New York City, under supervision of Prof. dr. Narula. After finishing his research, he started clinical work at the cardiology department of the St. Antonius Hospital in Nieuwegein in September 2013. 



\section{Publications}

M Versteylen, I Joosen, B Kietselaer, J Wildberger, H Crijns, L Hofstra. Gender difference in the prognostic value of cardiac computed tomographic angiography? JACC Cardiovasc Imaging 2013 (accepted for publication)

IF 6.2

J Borissoff, I Joosen, M Versteylen, A Brill, T Fuchs, A Savchenko, M Gallant, K Martinod, H ten Cate, L Hofstra, H Crijns, D Wagner, B Kietselaer. Elevated levels of circulating DNA and chromatin are independently associated with severe coronary atherosclerosis and a prothrombotic state. Arterioscler Thromb Vasc Biol. 2013; 33: 2032-2040. doi:10.1161/ATVBAHA.113.301627 IF 6.3

M Versteylen, B Kietselaer, P Dagnelie, I Joosen, A Dedic, R Raaijmakers, J Wildberger, K Nieman, H Crijns, W Niessen, M Daemen, L Hofstra. Additive value of semiautomated quantification of coronary artery disease using cardiac CT angiography to predict for future acute coronary syndrome. J Am Coll Cardiol 2013; 61(22): 2296-2305. doi:10.1016/j.jacc.2013.02.065

IF 14.1

J Borissoff, I Joosen, M Versteylen, H Spronk, H ten Cate, L Hofstra. Accelerated in vivo thrombin formation independently predicts the presence and severity of CT angiographic coronary atherosclerosis. JACC Cardiovasc Imaging 2012; 5(12): 1201-10. doi:10.1016/j.jcmg.2012.01.023

IF 6.2

I Joosen, F Schiphof, M Versteylen, E Laufer, M Winkens, P Nelemans, J Kooman, L Hofstra, J Wildberger, T Leiner. Relation between mild to moderate chronic kidney disease and coronary artery disease determined with coronary CT angiography. PLoS ONE 2012; 7(10): e47267. doi: 10.1371/journal.pone.0047267

IF 3.7

B Weijs, R Pisters, R Haest, J Kragten, I Joosen, M Versteylen, C Timmermans, L Pison, Y Blaauw, L Hofstra, R Nieuwlaat, J Wildberger, H Crijns. Pa- 
tients originally diagnosed with idiopathic atrial fibrillation more often suffer from insidious coronary artery disease compared to healthy sinus rhythm controls. Heart Rhythm 2012; 9(12): 1923-9. doi:10.1016/j.hrthm.2012.08.013

IF 5.0

A Mingels, I Joosen, M Versteylen, E Laufer, M Winkens, J Wildberger, M van Dieijen-Visser, L Hofstra. High-sensitivity cardiac troponin T: risk stratification tool in patients with symptoms of chest discomfort. PLoS ONE 7: e35059. doi:10.1371/journal.pone.0035059

IF 3.7

M Versteylen, R Takx, I Joosen, P Nelemans, M Das, H Crijns, L Hofstra, T Leiner. Epicardial adipose tissue volume as a predictor for coronary artery disease in diabetic, impaired fasting glucose and non-diabetic patients presenting with chest pain. Eur Heart J Cardiovasc Imaging 2012; 13: 51723. doi:10.1093/ehjci/jes024

IF 2.4

M Versteylen, I Joosen, M Winkens, E Laufer, R Snijder, J Wildberger, H Crijns, J Narula, L Hofstra. Combined use of exercise electrocardiography, coronary calcium score and cardiac CT angiography for the prediction of major cardiovascular events in patients presenting with stable chest pain. Int J Cardiol 2013; 167: 121-125. doi:10.1016/j.ijcard.2011.12.016

IF 5.5

M Versteylen, S Bekkers, M Smulders, B Winkens, C Mihl, M Winkens, T Leiner, J Waltenberger, R Kim, A Gorgels. Performance of angiographic, electrocardiographic and MRI methods to assess the area at risk in acute myocardial infarction. Heart 2012; 98: 109-115. doi:10.1136/heartjnl-2011-300185

IF 5.0

M Versteylen, I Joosen, L Shaw, J Narula, L Hofstra. Comparison of Framingham, PROCAM, SCORE, and Diamond Forrester to predict coronary atherosclerosis and cardiovascular events. J Nucl Cardiol 2011; 18: 904-11. doi:10.1007/s12350-011-9425-5

IF 2.8 
M Versteylen, I Joosen, L Hofstra. Monocoronary system with malignant course of the LAD. Heart 2010; 96: 1049. doi:10.1136/hrt.2010.198184 IF 5.0

\section{Research correspondence}

M Versteylen, I Joosen, L Hofstra. Reply; quantification of atherosclerotic coronary plaque: the missing link between elevated biochemical markers and adverse outcomes in the "vulnerable" patient? J Am Coll Cardiol 2013 (accepted for publication)

I Joosen, M Versteylen, M Das, B Kietselaer. Letter in response to Andrea K.Y. Lee et al. "normalcy rate of computed tomographic coronary angiography". Int J Cardiol 2012; 158(2): 299-300. doi:10.1016/j.ijcard.2012.04.116

M Versteylen, S Bekkers, M Smulders, R Kim, A Gorgels. The authors' reply. Heart 2012; 98(16): 1257-8. doi:10.1136/heartjnl-2012-302269 